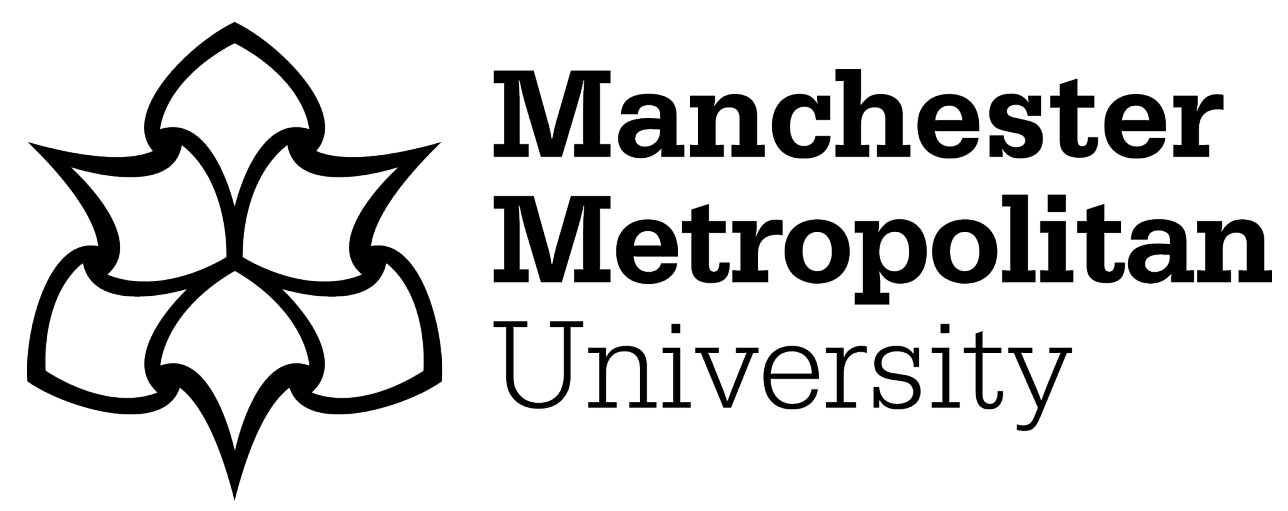

Almobarak, Fhaied Khalaf (2016) Exploring the perspectives of nurses, physicians, and healthcare administrators in Saudi Arabian hospitals on palliative care and palliative care nursing. Doctoral thesis (PhD), Manchester Metropolitan University.

Downloaded from: https://e-space.mmu.ac.uk/621247/

Usage rights: Creative Commons: Attribution-Noncommercial-No Derivative Works 4.0

Please cite the published version 
EXPLORING THE PERSPECTIVES OF NURSES, PHYSICIANS, AND HEALTHCARE ADMINISTRATORS IN SAUDI ARABIAN HOSPITALS ON PALLIATIVE CARE AND PALLIATIVE CARE NURSING

F K ALMOBARAK

PhD 2016 


\section{EXPLORING THE PERSPECTIVES OF NURSES, PHYSICIANS, AND HEALTHCARE ADMINISTRATORS IN SAUDI ARABIAN HOSPITALS ON PALLIATIVE CARE AND PALLIATIVE CARE NURSING}

FHAIED KHALAF ALMOBARAK

A thesis submitted in partial fulfilment of the requirements

of the

Manchester Metropolitan University for the degree of

Doctor of Philosophy

Department of Nursing

The Manchester Metropolitan University

$$
\text { July, } 2016
$$




\section{Abstract}

This thesis presents a qualitative research study, which identifies issues regarding the development of palliative care in the Kingdom of Saudi Arabia (KSA) hospital setting, from the perceptions of healthcare professionals working in Saudi Arabian hospitals.

The literature suggests that challenges to palliative care in KSA exist on various levels. At the professional level, they exist where healthcare practitioners may neither be sufficiently equipped nor sufficiently oriented to deal with palliative care concerns. Saudi practitioners may have misconceptions that lead them to recommend or carry out activities that may be detrimental to the palliative care of their patient. Issues also exist at institutional levels, where palliative care systems in place in Saudi hospitals are insufficient for addressing the needs of its patients. There are also issues found at the cultural and legal levels, where pain and individual choice are not considered sufficiently important in Islamic law especially when weighed against matters about prolonging and preserving life and preparing the individual for the afterlife.

The aims of this study were to determine the perceptions of KSA hospital healthcare professionals regarding palliative care, identify issues regarding the development of palliative care in the KSA hospital setting and to develop recommendations for strengthening the value of palliative care among healthcare professionals in KSA. To this end, semi-structured qualitative interviews were 
carried out with members of staff working in hospitals in KSA, with terminally ill patients.

The findings suggest that while some participants define palliative care in a way which is in keeping with internationally recognised definitions, others had not heard of the term, or defined it in a way which was incomplete. Insights are provided in to doctors and nurses experiences of palliative care, an example of which is that the personality of the healthcare provided was a significant factor in the quality of palliative care. The findings suggested that as a specialism, palliative care is seen as unique and growing, and participants understood the need and importance of this area. Issues and problems with palliative care were identified, including issues with communication and lack of material and human resources. Various opinions were offered as to the role of the media in promoting palliative care in KSA, issues with medication, including access and procedures, were discussed, and finally the participants spoke about their vision of the future of palliative care in KSA, and what would be needed to achieve this vision.

The contribution of the present study to the understanding of the perceptions of healthcare workers in KSA towards palliative care is significant considering the scarcity of research in this area, particularly qualitative research. It supports the findings of previous research into palliative care in KSA and the wider research on palliative care. It also provides a unique insight into the views of healthcare workers in an Islamic culture, expanding upon current literature. 


\section{Acknowledgements}

“O, my Sustainer! Open up my heart and make my task easy for me, and loosen the knot from my tongue so that they might fully understand my speech." (Quran 2528:114). I thank God Almighty for giving me the strength and the determination to complete this work.

I would like to send my thankfulness to my Director of Studies Professor Janet Marsden for her encouragement, patient guidance and useful critique of this PhD research. I am likewise extremely grateful for the direction and interminable backing from my second director Professor Duncan Mitchell. My special thanks to Dr Kate Fryer for her support.

I warmly thank my mother and father for their care and encouragement throughout my life. I would like to thank my wife for standing beside me throughout my study and while writing this research. I also would like to thank my whole family: sisters, brothers, my son and my daughter for everything positive I have received from them that contributed to this success.

Lastly, I would like to thank everyone who contributed to the completion of this work and those whom I have forgotten to mention. 


\section{Publication arising from the thesis}

Published journal article

Almobarak, F. (2014) 'Exploring the perspectives of nurses, physicians, and healthcare administrators in Saudi Arabian hospitals on palliative care and palliative care nursing'. Journal of Health Specialties, 2(2) p.54. 


\section{Table of Contents}

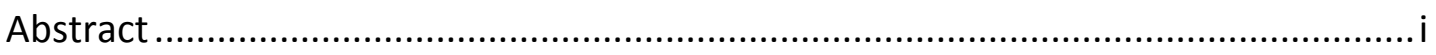

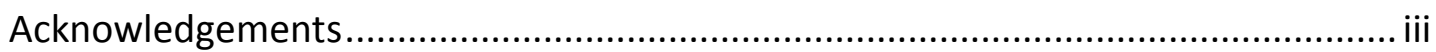

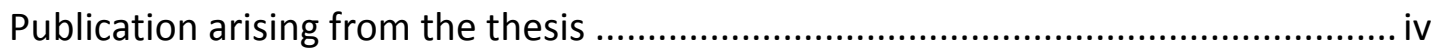

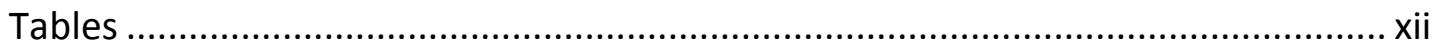

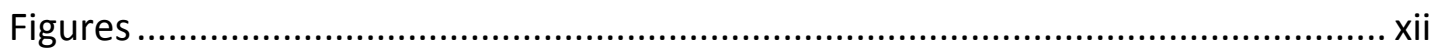

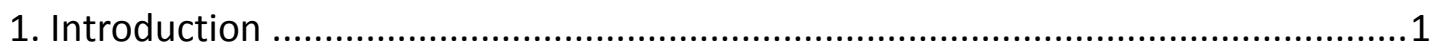

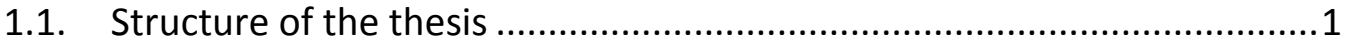

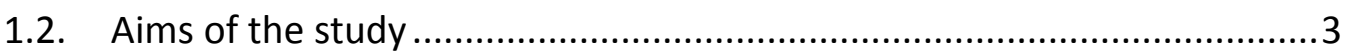

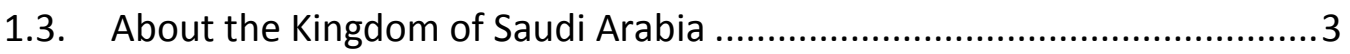

1.3.1. Culture, religion and politics................................................4

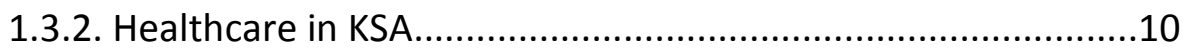

1.3.3. History of healthcare in KSA...............................................11

1.3.4. Limitations........................................................................14

1.3.5. Foreign workers and Saudization..........................................19

1.4. Motivations for the research ..........................................................2 21

1.4.1. About the researcher........................................................21

1.4.2. My personal palliative care philosophy.................................25

1.4.3. What is it like to work in healthcare in KSA? .........................25

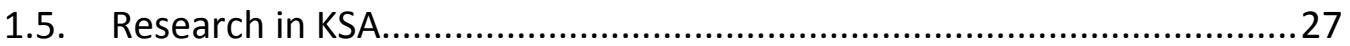

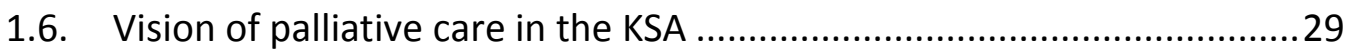

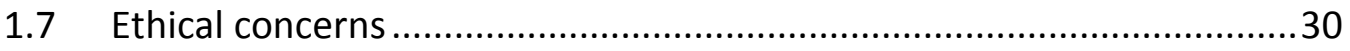

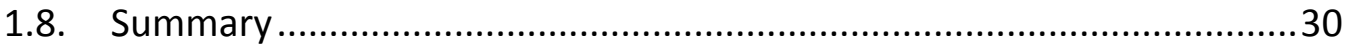

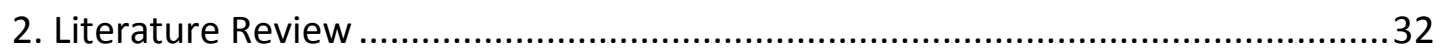

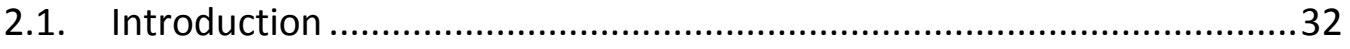

2.2. Literature review search strategy ...................................................... 32

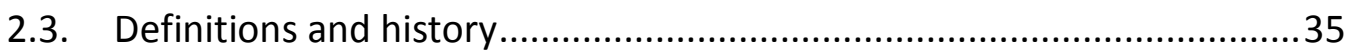


2.3.1. Policy and definitions of and within palliative care. .35

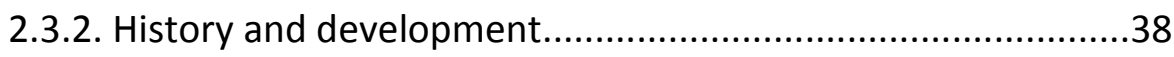

2.4. Prospective lessons for palliative care ............................................... 44

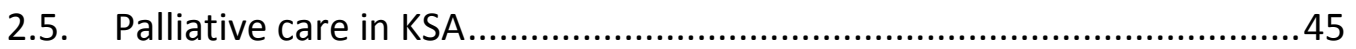

2.6. Background to palliative care in KSA ....................................................46

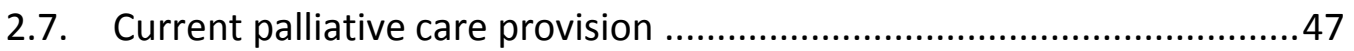

2.8. Evaluation of palliative care in the Middle East .....................................50

2.8.1. Time between referral and death........................................52

2.8.2. Time between last chemotherapy and death (TLCD).............55

2.8.3. Use of antimicrobials...........................................................56

2.8.4. Terminal hospital length of stay........................................56

2.9. Summary of quality indicator evidence ...............................................5

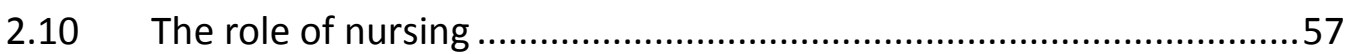

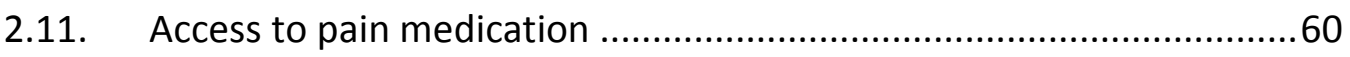

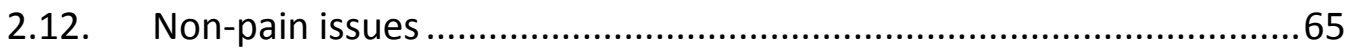

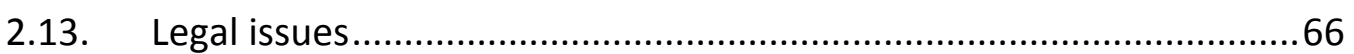

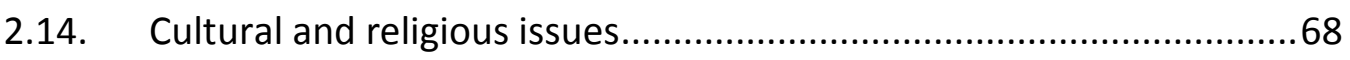

2.15. Islamic perspective on illness and death ..........................................69

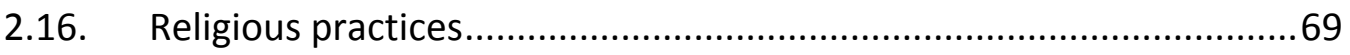

2.17. The family and 'truth-telling' ........................................................... 71

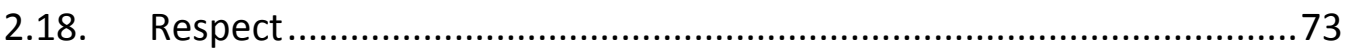

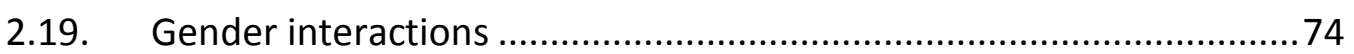

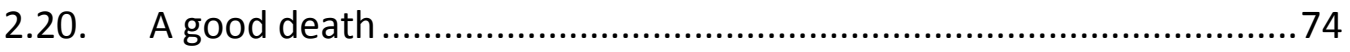

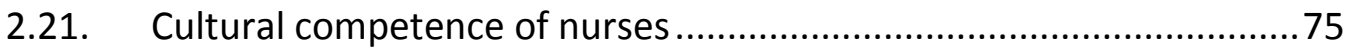

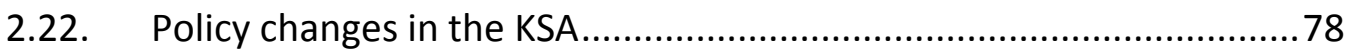

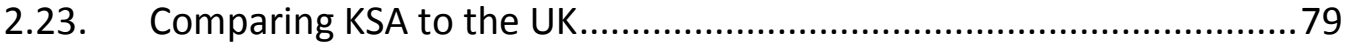

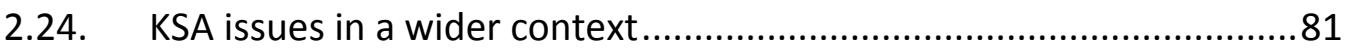

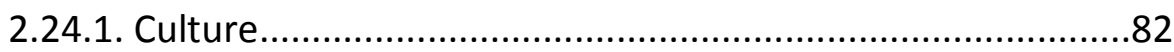

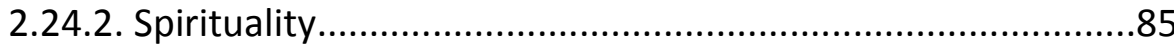

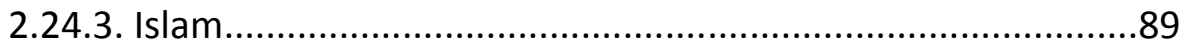

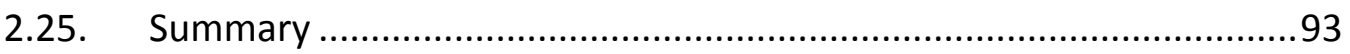




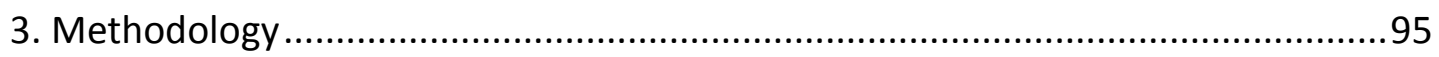

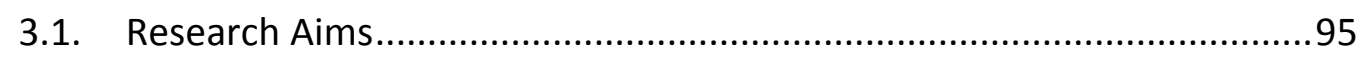

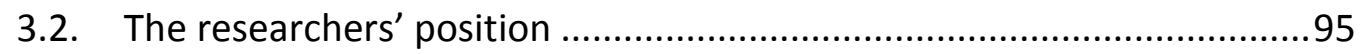

3.3. Paradigm, theoretical perspectives and methodology ..........................98

3.3.1. Paradigm: subjectivist leading to qualitative research............98

3.3.2. Theoretical perspectives: symbolic interactionism and

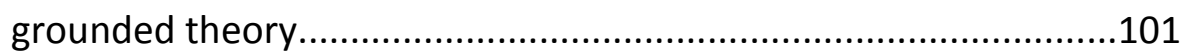

3.3.3. Methodology: pragmatic and thematic analysis...................106

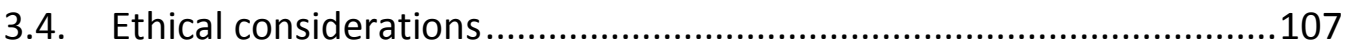

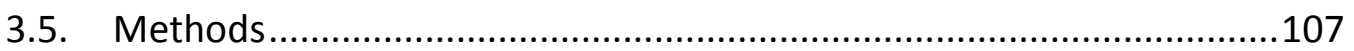

3.5.1. Manchester Metropolitan University sampling...................109

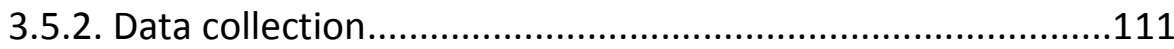

3.5.3. Data analysis.....................................................................113

3.5.4. Interpretation and translation issues.................................117

3.5.5. Trustworthiness of the data.............................................119

3.6. Ensuring quality in qualitative research ..........................................120

3.7. Conducting qualitative research in the KSA ...................................123

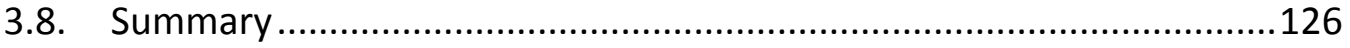

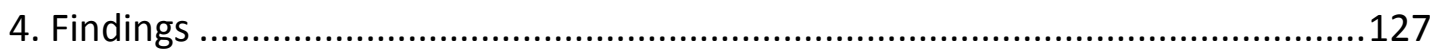

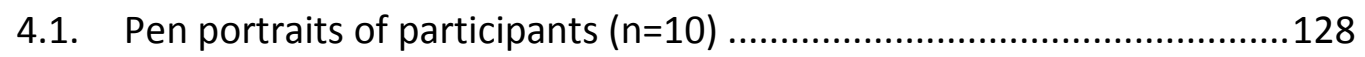

4.2. Outline of the themes and subthemes ................................................129

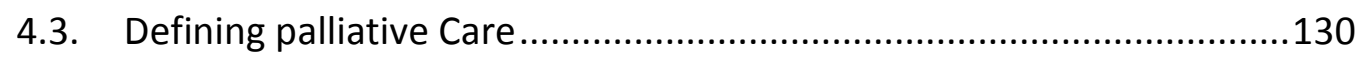

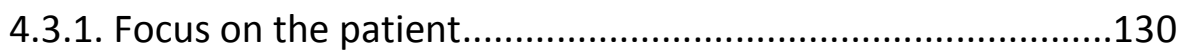

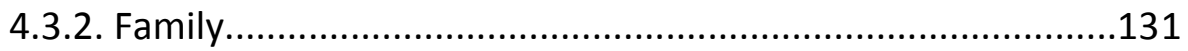

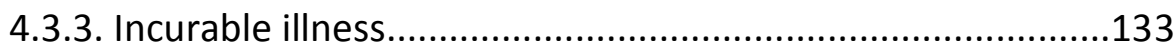

4.3.4. References to death, dying and end of life.........................134

4.3.5. Holistic view...................................................................135

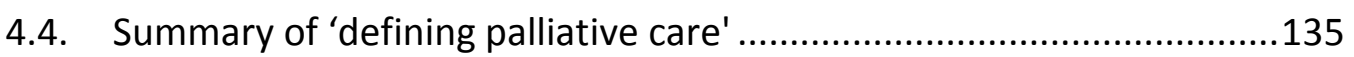

4.5. Perceptions of palliative care as a medical specialisation.....................136 
4.5.1. Palliative care as a unique or rare specialisation. .137

4.5.2. As a growing speciality in demand. .139

4.5.3. The need and importance of palliative care. .139

4.6. Summary of palliative care as a medical speciality 144

4.7. Doctors and nurses 144

4.7.1. Perceptions of palliative care. .145

4.7.2. Palliative care as team. 145

4.7.3. Personal qualities. 146

4.8. Summary of 'doctors and nurses' 148

4.9. Issues and problems in palliative care. 148

4.9.1. Communication. .149

4.9.2. Language barriers 149

4.9.3. Lack of resources. 150

4.9.4. Not a lack of resources, but of poor allocation .152

4.10. Summary of 'issues and problems' 153

4.11. Knowledge, training and education .155

4.11.1. Need for palliative care education .156

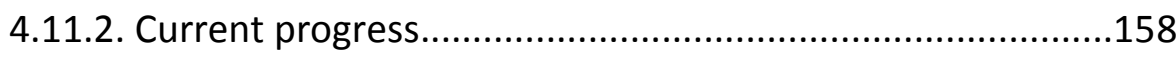

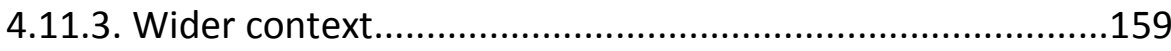

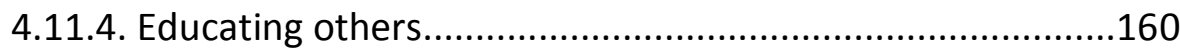

4.12. Summary of 'knowledge, training and education' ..........................161

4.13. The role of media in palliative care.............................................161

4.13.1. Media as having an important role.....................................162

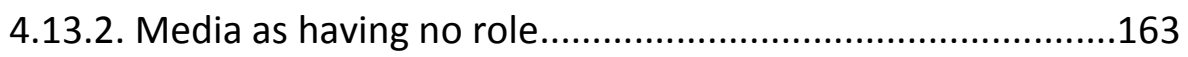

4.13.3. Media as having a negative role........................................164

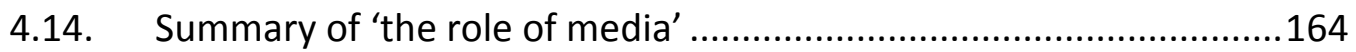

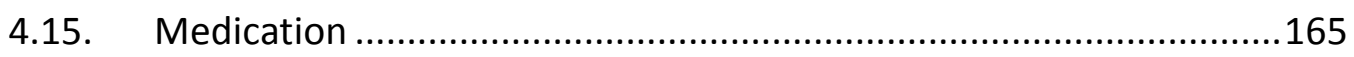




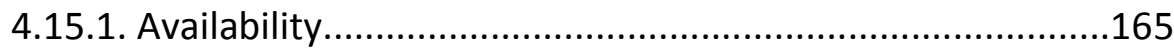

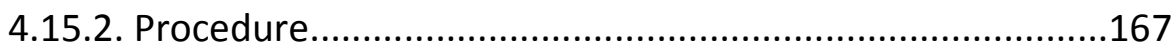

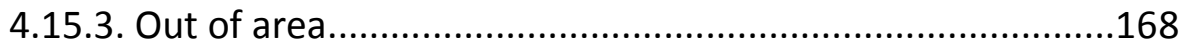

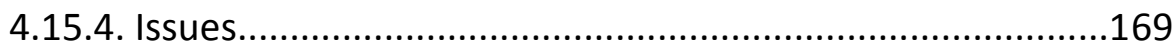

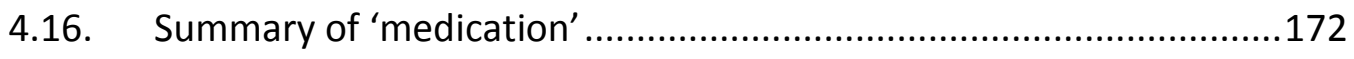

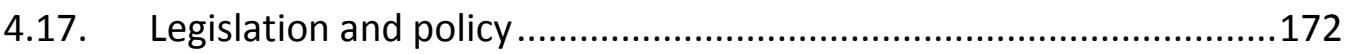

4.17.1. Legislation...................................................................172

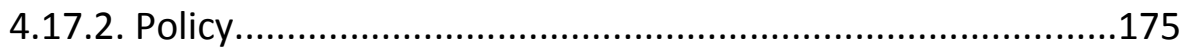

4.18. Summary of 'legislation and policy' ...............................................178

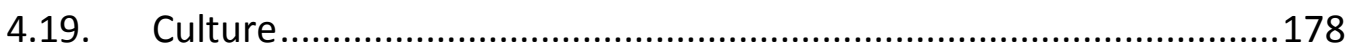

4.19.1. Truth telling............................................................... 178

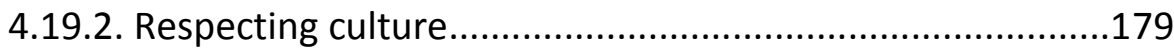

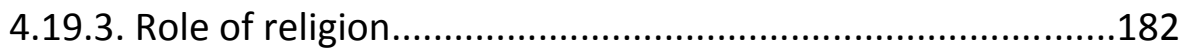

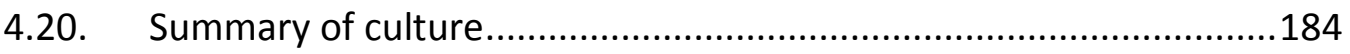

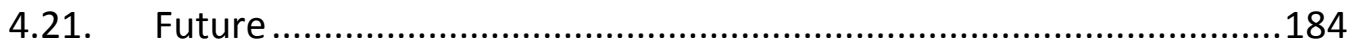

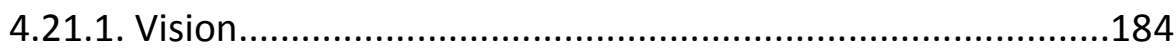

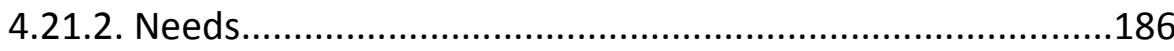

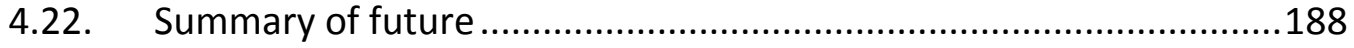

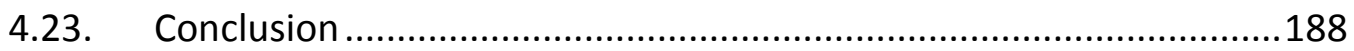

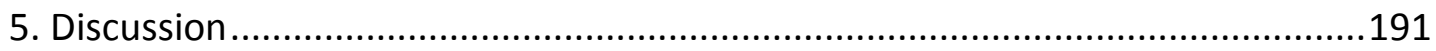

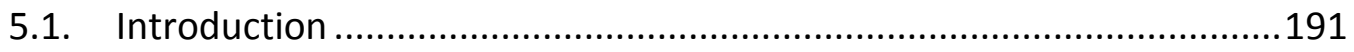

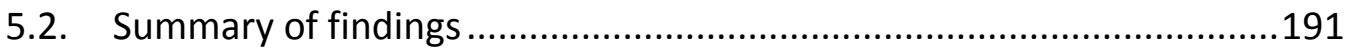

5.3. Comparison of findings to literature review .....................................192 
5.3.1. Defining Palliative Care........................................................192

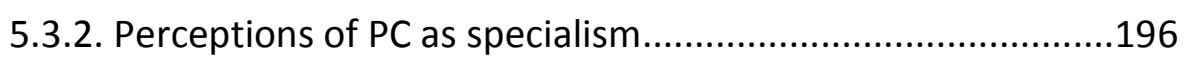

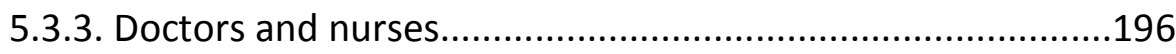

5.3.4. Issues and problems.........................................................197

5.3.5. Knowledge, training and education....................................200

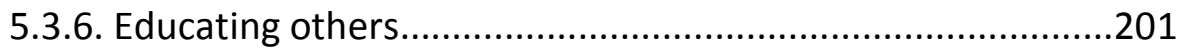

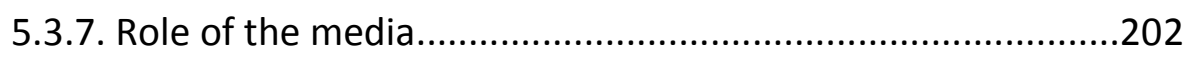

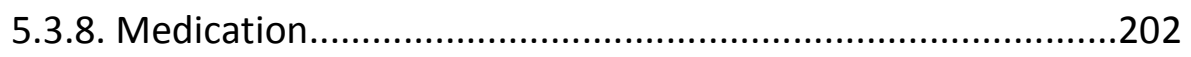

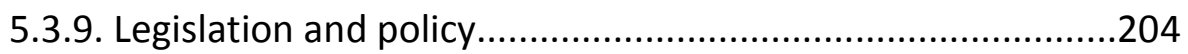

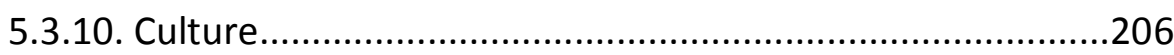

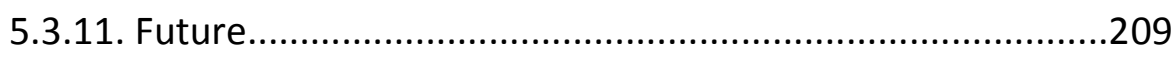

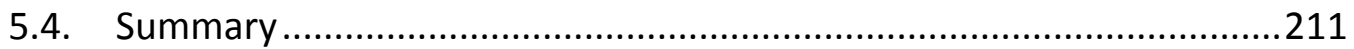

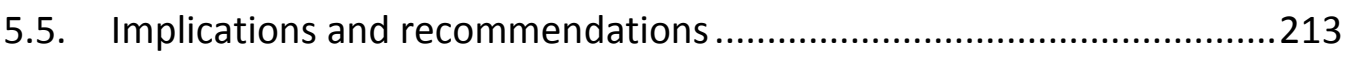

5.5.1. For the future of palliative care in KSA..............................213

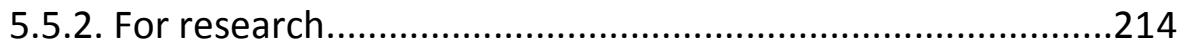

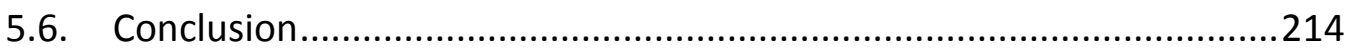

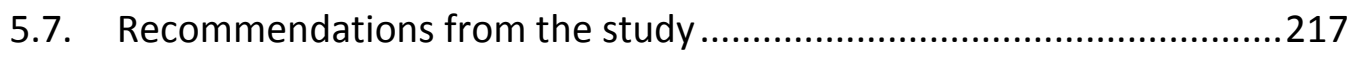

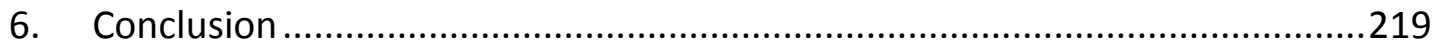

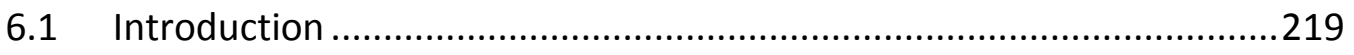

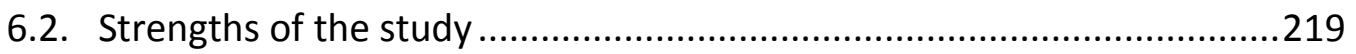

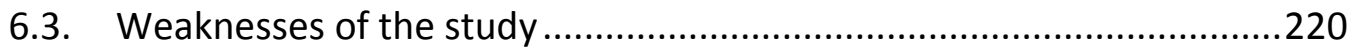

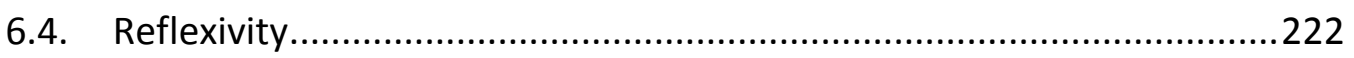

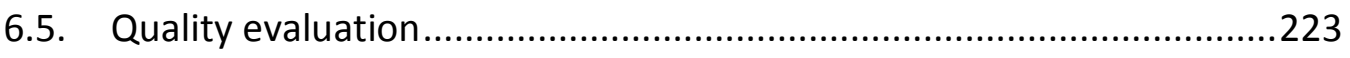

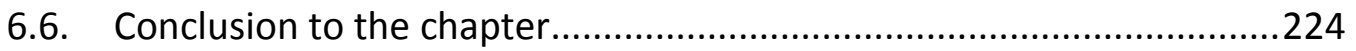

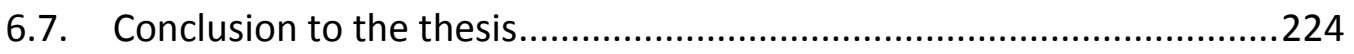

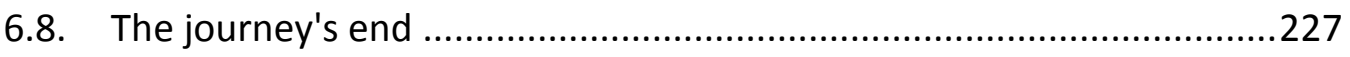

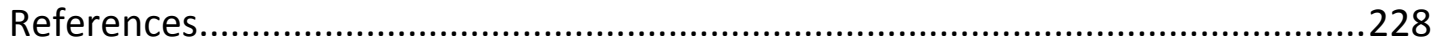

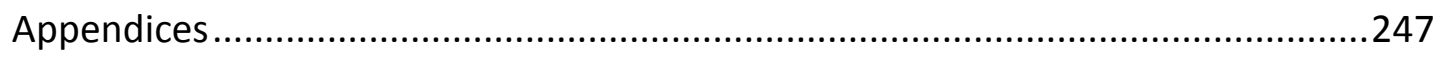

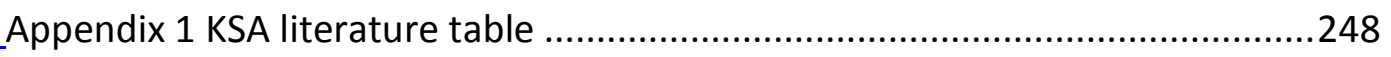

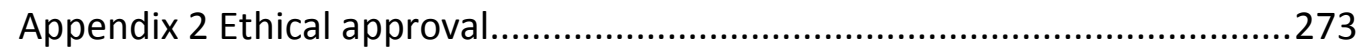

Appendix 3 Participant information sheet English ......................................225 
Appendix 4 Participant information sheet Arabic 277

Appendix 5 Participant consent form English ..............................................279

Appendix 6 Participant consent form Arabic .................................................280

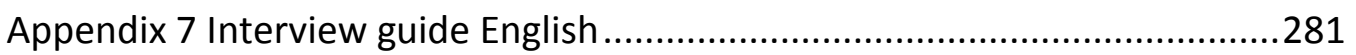

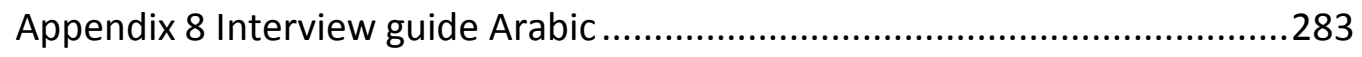




\section{Tables}

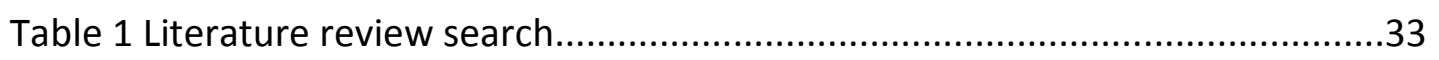

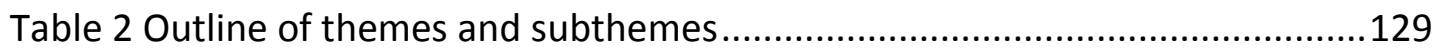

\section{Figures}

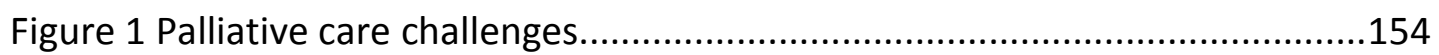




\section{Introduction}

This thesis presents a qualitative research study, which identifies issues regarding the development of palliative care in the Saudi Arabia hospital setting, from the perceptions of healthcare professionals working in Saudi hospitals. In the context of this study, palliative care is defined as a multidisciplinary approach that is specialized towards the care of people with serious illnesses, particularly in terms of alleviating the pain and stress experienced by such people (National Institute for Health and Care Excellence, 2004). The aim of this chapter is to introduce the reader to the thesis, by first outlining the structure of the thesis and the aims of the research. The chapter then sets out to provide essential background information on the Kingdom of Saudi Arabia and its healthcare system, explain the ethical implications of undertaking research in this environment, describe the researchers background and finally, provide the motivations for the research. While the thesis is written in the third person, for ease of articulation, the first person has been used for the description of the researcher's background.

\subsection{Structure of the thesis}

This chapter is followed by:

\section{Chapter 2: Literature Review}

This chapter details the definitions, history and development of palliative care as a concept, before providing a complete review of the literature of palliative care within KSA. A comparison is made with the United Kingdom (UK) situation before 
salient issues are discussed in reference to international literature. This will place the current research within the wider research landscape.

\section{Chapter 3: Methodology}

This chapter will describe the theoretical foundations of the research, and the processes used to carry out the research, including a discussion of quality in qualitative research. This demonstrates the firm foundations in which the findings are grounded. Finally, recruitment of research subjects, understanding the sample and the research ethical concerns are discussed.

\section{Chapter 4: Findings}

This chapter begins with pen portraits of the participants, followed by a visual representation of the themes and sub-themes which were identified during analysis of the interviews. The themes and sub-themes are then described.

\section{Chapter 5: Discussion}

This chapter will evaluate the research and findings, both in the light of the methodology and methods and by comparison to the literature discussed in chapter 2. This will culminate in recommendations for clinical practice and research.

The chapter concludes by reflecting on the whole thesis. It reiterates the research questions and how they were answered and gives a summary of the most significant findings and how the findings relate to current literature

\section{Chapter 6: Conclusion}


This chapter concludes the thesis by providing an overview of the strengths, weaknesses and provides reflection on the research and research journey.

\subsection{Aims of the study}

The aims of this study were threefold:

- To determine the perceptions of KSA hospital healthcare professionals toward palliative care.

- To identify issues regarding the development of palliative care in the KSA hospital setting.

- To develop recommendations for strengthening the value of palliative care among healthcare professionals in KSA.

To this end, semi-structured qualitative interviews were carried out with members of staff working with terminally ill patients in hospitals in KSA.

\subsection{About the Kingdom of Saudi Arabia}

This research is based in the Kingdom of Saudi Arabia (KSA), a country different in many ways to the UK. Therefore, it is necessary to provide some background information about the country, to provide essential context for the research. Firstly, this thesis will describe the cultural, religious and political climate of KSA, and the potential impact of that on the development of palliative care, before detailing the development of the healthcare system, its current state, limitations and future challenges. The thesis will then reflect upon issues which affect the experience of working in healthcare in KSA and finally outline the research situation in relation to palliative care. 
KSA is a conservative Muslim country, and the true culture of KSA is not well documented. Therefore, much of the background information provided is based on the authors own knowledge and experience, as a native of KSA.

\subsubsection{Culture, religion and politics}

KSA is the largest country in the Arabian Peninsula and was founded as a state in 1932. The population of KSA is approximately 27 million, $20 \%$ of which is non-native (Albaqawi, 2015). $5.4 \%$ of the female population and $4.5 \%$ of the male population are over 60 (United Nations Statistics Divisions, 2015). Female life expectancy is 78 years, and male life expectancy is 74 years (WHO, 2015).

The Kingdom is the world's largest oil producer (Organization of Petroleum Exporting Countries, 2016) and as such, it is categorized as a high-income economy with the $19^{\text {th }}$ highest GDP in the world (Quandl, 2016). The economy is mainly supported by the oil industry, which accounts for more than $95 \%$ of all exports and $70 \%$ of all government revenue (Falquero, 2016). Therefore, Saudi Arabia can be considered a wealthy country, although as a native of Saudi Arabia, it is clear to see that wealth is not evenly distributed, and despite the governments' commitment to free healthcare for all, the quality of healthcare received in general, as well as in specialist and developing areas such as palliative care, as discussed within this thesis, varies widely.

The official religion of KSA is Islam (Bureau of Experts at the Council of Ministers 1992), and the indigenous population is officially 100\% Muslim (NationMaster, 2016). This has impacted upon the development of palliative care in KSA. The 
culture of KSA derives from 1435 years of Islamic heritage, its historic role as a centre of trade in the world (Mecca), and its Bedouin customs and traditions. Saudi society has faced rapid growth over the past fifty years. Many Saudi people have kept their values, traditions and customs and this can be seen in the hospitality provided by most people, and even the style of dress. Tradition is important to most Saudi people, even when it is merged into the modern world. Saudi traditions come from Arab customs and Islamic teachings which are taught at an early age with family and in formal education. Traditions are ancient, and it is culturally important that they are respected (Royal Embassy of Saudi Arabia, 2016). Such factors have an impact on the delivery of healthcare, and this may be applied even more so in palliative care, given the role of religion and culture in defining and shaping experiences and expectations of death and dying. This will be further explored in the literature review.

To understand the way that Islam may have influenced the development of palliative care in KSA, it is helpful to understand some basic information about Islam. One of the three monotheistic world religions, it can be best summarised by its six tenets of faith, and five pillars of practice.

The six tenets of faith are:

- Belief in Allah (God).

- Belief in the angels, including Jibril (Gabriel) and the angels of death.

- Belief in the revealed books, including the Torah, the Old and New Testament, and the Qur'an. 
- Belief in the commissioned Messengers (peace be upon them all), including Ibrahim (Abraham), Musa (Moses), Isa (Jesus) and Muhammad as the final messenger.

- Belief in the resurrection of all souls after death and the events of the day of judgement.

- Belief in the predestination by Allah of all things, both the (seemingly) good and the (seemingly) bad.

The pillars of Islamic practice are:

- The Shahada (declaration of faith), which is that there is no god but Allah, and Muhammad (peace be upon him), is his final prophet and messenger. This is stated by one wishing to become Muslim, and repeated by all Muslims several times a day within the prayer.

- Salah, the prayer, Muslims pray five times a day.

- Zakaat, the giving of a proportion of one's wealth to charity in order to purify the remainder of it.

- Sawm, fasting during the month of Ramadan.

- Hajj, performing the pilgrimage to Makka, in KSA, at least once in one's lifetime if one has the resources.

(Bahammam, 2012)

While the foundations of the Islamic faith presented above are universal and unchanging, the expression of Islam varies between countries, cultures and 
individuals. KSA is a conservative Muslim country, with a system of law based on the Islamic Shariah, with the Qur'an as its constitution (Al-Farsy, 1990). The founding of Saudi Arabia was an alliance between Imam Mohammed Bin Saud and Imam Mohammed Bin Abdul Wahhab (Saudi Commission for Tourism and National Heritage, 2013), and KSA is still aligned with the Wahhabi school of thought, which is noted for its conservatism. While not all citizens of KSA will have the same interpretation of Islam, most people publically adhere to the dominant view. It is important to take this into account when considering healthcare in KSA, especially as healthcare is delivered by a multi-cultural workforce. Again, this will be discussed in detail in the literature review.

An area of Saudi culture, based on Islam, which is often seen to be at odds with Western thought, is the differing gender roles and relationship between the genders. The gender situation in Saudi Arabia is based on a strict interpretation of the Islamic Shariah. Unlike the predominant Western view that men and women's choices should not be dictated by gender, Islam, while stern in its acknowledgement of the equal value of the sexes, who worship in the same way, dictates different roles to men and women (Shakir, 2008). Within a family, the man is seen as the head of the household, who should consult with other members of the family, including females, and make final decisions. Within traditional Islamic culture, the influence of the women within the family becomes invisible outside of the home, as in this environment the man will represent the family. However, Islamic families can be considered both patriarchal and matriarchal, and the women 
may have considerable power within the family, "In the house the women-especially the mother-rule supreme"(Schimmel, 1992).

Muslim women have historically held academically and politically important roles (Schimmel, 1992). However, Islam gives equal weight to the role women play as mothers and maintainers of the home. The woman is viewed as the first teacher, and raising her children is a respected job (Shakir, 2008). Historically few Saudi women have been in paid employment, partly due to strict gender segregation. However, recent policy changes have allowed an increased number of women to enter the workforce (von Mittelstaedt and Shafy, 2015). Islam dictates modest dress for men and women, with differences in the details, reflecting the differing physical attributes and natures of men and women. While the exact expression of this varies between cultures, most women in KSA wear full covering including their faces (von Mittelstaedt and Shafy, 2015). Given the role of the family around the time of the patients' death, such cultural norms will have a significant impact on the way palliative care is delivered and received.

It is also helpful to understand the structure of the legal system in Saudi Arabia, which will impact upon decision making around aspects of palliative care. The legal system in Saudi Arabia is based on 'Shariah'. Shariah is concerned with all aspects of life experienced by people, such as justice, transactions, knowledge, worship, values and principles. Shariah is derived from the Qur'an, first, and Sunnah (the sayings of Prophet Muhammad), second. The Qur'an is the word of God according to the Prophet Muhammad (peace be upon him), and the Sunnah are the sayings of 
and practices adopted throughout his life. Thirdly, (ljma) is the consensus of Muslim Scholars on cases and issues before the death of the Prophet, meaning that where there are some differences of opinion, the consensus of the religious scholars is followed. The fourth source of law is (Qiyas) analogy, with is a method of applying the principles used to arrive at rulings for issues before the death of the Prophet, to issues which emerge after the death of the Prophet (The Islam Project, 2016). Many of KSAs' laws which impact upon palliative care will be based on Qiyas, for example, the permissibility of certain medications.

KSA is a monarchical state, and the pinnacle of the government is the King (Bureau of Experts at the Council of Ministers 1992). The government is made up of 22 ministries, and each ministry represents a department of the government, such as health, education and finance. The Consultative Council (Majlis Al-Shura) provides advice to the King as a legislative body. The Council suggests new regulations and laws and modifies existing laws. The Council contains 150 members who are chosen by the King for a 4-year period which can be revived with a royal decree. KSA is split into 13 provinces (Manatiq), with a governor (Ameer) and a deputy governor of every province. In these provinces, there are Councils that suggest and advise the governor about the development of the province. The King is at the top of the legal system. He is the final person to appeal to, and pardons are only issued by him. The largest courts in the KSA are the Shariah Courts. Ultimately, the King is the final court (Royal Embassy of Saudi Arabia, 2016). 
The Majlis Al-Shura specializes in providing advice to the King on cases and issues. The Council formulates various committees, members of which are determined according to experience. There are 12 committees concerned with the following areas: health, education, culture, finance, security, Islam, information, social affairs, economy, industry, administration, foreign affairs, human rights, services and public utilities. The role of the Majlis Al-Shura is to suggest new legislation as well as reviewing present laws. The Majlis Al-Shura ask the government officials to participate in important meetings and get access to government documents (Royal Embassy of Saudi Arabia, 2016). The literature review discusses how Islamic law and palliative care interact and conflict.

This section has provided a brief background to KSA, a country which differs markedly from the UK and other Western countries, and is, in fact, unique in the world due to its creation and development. It has demonstrated how the unique elements of KSA may have influenced the development of palliative care in the country, and this will be further explored in reference to the current literature in the Literature Review Chapter.

\subsubsection{Healthcare in KSA}

In the UK, United States of America (USA) and other countries where palliative care is well established, its development has been clearly influenced by the existent structure of health and social care in the country, as will be demonstrated in the literature review. Equally, in Saudi Arabia, the development of palliative care takes place within the healthcare structure present in the country and is influenced by its limitations. The history, current status, limitations and future directions of 
healthcare in Saudi Arabia are described below, to present the context in which the palliative care in KSA exists and must be understood. In addition, the specific issue of Saudization (the required increasing of KSA nationals into the workforce), which has a great impact on healthcare will be discussed, and finally, an expose of the research situation in KSA.

\subsubsection{History of healthcare in KSA}

The Ministry of Health(MOH) is the main provider of health services in KSA and was established in 1954 to provide free healthcare to all Saudi nationals (Albaqawi, 2015). Later this was expanded in include pilgrims. As a result of free healthcare, diseases such as smallpox and malaria were eliminated, infant mortality rate was reduced, and life expectancy significantly increased (World Health Organisation, 2013). From 1970 to 1989 KSA focused on the establishment of the infrastructure of hospitals, dispensaries, clinics, pharmacies and health laboratories (Royal Embassy of Saudi Arabia, 2016). After the establishment of these facilities, Saudi began to encourage people to join the field of medicine and started to attract foreign workers. They also imported technology from abroad and established relationships with specialized global hospitals.

The government bodies including the main provider, the $\mathrm{MOH}$, have established most of the health institutions in the country. The government has encouraged private sector involvement by offering loans for establishing hospitals, pharmacies and clinics. The loan is interest-free and long-term. The private sector accounted for $27 \%$ of healthcare provided in the country in 1990 (Ahmed, 2012). 
In the past, the curative model of care was used. However, this model is costly when diseases can be reduced through preventing them from happening. The $\mathrm{MOH}$, therefore, performed some preventative activities such as malaria and tuberculosis control. They selected a Primary Healthcare approach to apply preventative care (Almalki et al., 2011). A ministerial decree was issued in 1980 to establish Public Healthcare (PHC) centres, with the following aims:

1. Educating the public about health problems and how to prevent them.

2. Providing comprehensive women and child care.

3. Providing medication, and treatment of general diseases and immunization of children.

(Almalki et al., 2011).

In return, the numbers of patient visits to outpatient clinics had reduced by $82 \%$ by 2009. The PHC Centres used health records which helped to reduce the many consultations. Prescriptions in patients' files were used to improve the prescribing practices (Al-Mazrou, 2002).

\section{Current status of healthcare in KSA}

The Saudi healthcare system provides a basic platform of healthcare services to everyone, with advanced specialised treatment facilities accessible at some private and public hospitals (Ahmed, 2012). The Saudi government is committed to providing healthcare for every citizen (Bureau of Experts at the Council of Ministers 1992) and raised the amount of expenditure on the ministry from $2.8 \%$ in 1970 to $6.2 \%$ in 2009 (Almalki et al., 2011). The system is based on three levels, primary 
healthcare centres, secondary general hospitals, and regional referral hospitals.

There are also well-developed private hospital services ( Al-Shahri et al. 2004). Health services are provided by $\mathrm{MOH}$ at three levels:

1. Primary (preventive and curative health services in PHC)

2. Secondary (advanced care in public hospitals through referrals)

3. Tertiary (complex cases in central and specialised hospitals)

The $\mathrm{MOH}$ is the key government provider and financier of health care services in Saudi Arabia, with a total of 244 hospitals $(33,277$ beds) and 2037 primary health care centres, (Almalki et al., 2011). The MOH offers $60 \%$ of the total health services in Saudi Arabia. The other government agents involved in healthcare are:

- National Guard Health Affairs

- King Faisal Specialist Hospital and Research Centre

- Armed Forces Medical Services

- Ministry of Higher Education Hospitals (teaching hospitals)

- Security Forces Medical Services

- ARAMCO Hospitals

- Royal Commission for Jubail and Yanbu Health Services

- School Health Units of the Ministry of Education

- The Red Crescent Society

Collectively, the government agencies preside over 39 hospitals with a 
capacity of 10,822 beds (Ministry of Health, 2009). The private sector additionally contributes to the delivery of health care services, particularly in cities and large towns, with a total of 125 hospitals (11,833 beds) and 2,218 dispensaries and clinics (Almalki et al., 2011).

Yearly about two million pilgrims perform Hajj in Makkah. KSA is the heart of the Islamic world as the two holiest destinations in Islam that are located in KSA are Makkah and Madina. Seventy percent (70\%) of the pilgrims are from all over the world. Services provided to pilgrims are preventive and curative and emergency for all of them through quarantine services at airports and land ports (Royal Embassy of Saudi Arabia, 2016).

We can compare KSA to the UK, in that both countries provide healthcare to all which is free at the point of access. However, the unique development of Saudi Arabia, which gained a great deal of wealth very quickly through oil, is entirely different from the UK, and this has many implications for the way in which healthcare has developed, and therefore palliative care within this.

\subsubsection{Limitations}

Today, Saudis and non-Saudis are struggling to access healthcare within the thousands of hospitals and clinics that exist. Transformation of the healthcare system has been continuing but has not been able to meet the needs of the population (Almalki et al., 2011).

The establishment of a modern healthcare system in KSA was not based on a modernisation of the whole country but was centred upon the major cities, 
especially Riyadh, Jeddah and Dammam (Alkabba et al., 2012), which accounts in part for health inequalities evident in KSA. The delivery of quality services is far weaker in the suburbs and other main cities in KSA. The reality is that there is great inequality in the availability and quality of healthcare throughout KSA.

KSA increased the budget of health to113 billion Saudi Arabian Riyal (SAR) in 2011. However, in terms of the percentage spent on healthcare as a percentage of GDP, KSA still is behind many developed nations in the world (The World Bank, 2011). Furthermore, healthcare indicators of KSA, when looked at and compared to other developed countries such as Europe, show that there is a shortage of beds and healthcare professionals such as doctors and nurses. The shortage is common across all the Gulf countries. However, KSA has the lowest number of beds, nurses and doctors per population inside the Gulf Cooperation Council(Ministry of Health, 2009). With the increasingly limited access to $\mathrm{MOH}$ hospitals, many people seek care at private hospitals. Specialised medical care can be difficult to access, with complicated and inconsistent eligibility criteria. Complicated surgical procedures such as open heart surgery are done in major city hospitals by health professionals that do not always meet the international standards (Ahmed, 2012).

One of the major costs for healthcare providers is spending on qualified medical staff. A large number of physicians, nursing staff, and paramedical staff in KSA move to European nations and Western Countries after a few years due to better possibilities and training features. Additionally, the present Saudi rules for employment and Saudization continue to further increase the staff cost due to limited available resources. The government's role is extremely important in 
establishing career-focused educational institutions, such as medical and nursing colleges, to increase the supply of local medical professionals and to drive qualified Saudi talent into jobs. Moreover, to overcome supply gaps the government needs to provide funding to the private sector and improve employment regulations to be able to attract qualified resources from abroad (Ahmed 2012).

Palliative care, as it is not life-saving and its success is difficult to measure, is not usually a top priority for Saudi health providers. In the countries with the most established provision of palliative care, we can see that the whole healthcare structure is well developed. In a country such as KSA, where healthcare provision is struggling to meet even the basic needs of the population, we can expect palliative care to be a low priority for those with the power to allocate funds. The literature review will explore the evidence for this expectation.

Colliers International Healthcare $(\mathrm{CIH})$ conducted a study in 2012 using questionnaires and interviews and concluded the investors and operators' views regarding healthcare situation in KSA. Their findings were that the current healthcare sector is dominated by insurance companies ( 25 companies listed on the Saudi Stock Exchange) and is hence driven by cost rather than quality (Ahmed, 2012).

They also found that there has been a tremendous increase in lifestyle-related diseases such as diabetes, high blood pressure, being overweight, heart (cardiovascular) disease and kidney disease (needing dialysis). Presently, the private sector performs an important role in providing care for these illnesses. However, 
the government needs to take an effective role in prevention through educating people and offering preventive services.

The study suggests that implementation of Information Technology should have greater prevalence in the sector similar to other developed countries in which medical files for patients are either unified or centrally maintained. Improvement in the unified or centralised filing is expected to improve diagnosis and provision of healthcare services (Ahmed, 2012).

$\mathrm{ClH}$ also found that with the current population growth, access has been a major issue in public sector hospitals. This shortage has resulted in a rise in demand for private medical care. With exaggerated healthcare prices and the emergence of lifestyle-related illnesses, both private and public should make more partnerships to create better provision of care (Ahmed 2012). This sentiment was echoed by Alhusaini (2006), who noted that in spite of the multiplicity of healthcare providers, there is no clear coordination or communication between providers. This results in duplication of efforts, and a waste of resources and time (Alhusaini, 2006). There are numerous opportunities such as the presence of some of the staff who are well trained and qualified from different countries, laboratories and equipment, to take advantage of. The opportunities stay within each sector as the result of poor coordination. In contemplation of the provision of modern, comprehensive, organised, accessible, and fair healthcare, a royal decree, issued in 2002, asked for the establishment of the Council of Health Services to be governed by the Minister of Health and representatives of some other government sectors and private sectors. The Council's goal was to develop a policy to coordinate and integrate all 
health-care authorities in KSA. However, further progress is needed in this area (Alkhazem, 2009).

\section{Future directions}

KSA has a large and fast-growing population. CIH (2012) estimated that by 2016 the total population would be 30 million, of which over half will be natives. To maintain the network of healthcare facilities, the Saudi Government has applied a two-level health plan:

Level 1: a network of primary healthcare centres ( $\left.\mathrm{PHC}^{\prime} \mathrm{s}\right)$ and clinics established throughout the Kingdom.

Level 2: a network of secondary care hospitals and advanced treatment facilities (located only in major urban areas across the country).

(Ahmed, 2012).

According to KSA MOH (Ministry of Health, 2013), the ministry's vision is to deliver an integrated and comprehensive healthcare service according to the highest global standard and its mission is to provide all levels of healthcare, enhance public health, prevent diseases and to regulate the private and public health sectors while conducting research, delivering academic training and promoting health investment.

The Ministry detail their strategic objectives as:

-Delivering a comprehensive and integrated healthcare plan while maintaining a strong focus on research and education. 
-Establishing a culture of institutional work, enhancing quality levels, and ensuring performance measurement and monitoring.

-Attracting qualified personnel and developing Human Resources.

-Advancing e-Health and information systems.

- Optimizing the use of resources by applying healthcare economics.

Sebai et al. (2001), in reviewing healthcare in Saudi Arabia, set out the challenges for the future of the development of healthcare. These include the management of services which is multi-agency and not uniform and would benefit from better coordination, the financing of health services in the light of a rapidly growing population and declining government revenues, balancing curative and preventative activities, and the training and development of Saudi manpower, an issue which will be discussed in more detail in chapter two.

\subsubsection{Foreign workers and Saudization}

There are not enough fully trained and qualified healthcare professionals of Saudi nationality. Therefore a large proportion of healthcare providers are foreign workers. Foreign workers make up about $45.8 \%$ of the total healthcare workforce, and about $49.72 \%$ of the nursing workforce (Al-Homayan et al., 2013). Only $20 \%$ of physicians are Saudi Nationals (Royal Embassy of KSA 2015). The importing of foreign workers, primarily Westerners, is important in bringing the necessary qualifications and competencies to the system, which could not be sufficiently provided by a purely indigenous workforce. However, the reliance on an expatriate workforce may be preventing the government from providing native Saudis with the opportunities to develop the skills and experience to fulfil these roles. 
Regardless of the reason, this situation exists within the healthcare sector and is reflected throughout KSA. The unemployment rate for Saudi natives over the age of 15 was recorded as $11.7 \%$ in 2014 (Central Department of Statistics and Information, 2015). Fakeeh (2009), in his thesis on the Saudization policy within KSA, explains the high unemployment rate by the unique development of KSA over the last 100 years. KSA gained wealth rapidly in the second half of the twentieth century due to the discovery of oil reserves. It had the money, but not the skills, to build a modern state. Therefore important skills were imported by way of a foreign workforce. This mismatch between skills and demand has persisted, and the government response has been to introduction a policy of Saudization, which has as its focus the compulsory employment of a quota of Saudis for both private and public organisations. However, Fakeeh (2009) suggests that this policy falls short of solving the problem, as it does not address the mismatch between skills/education and the needs of the labour market. This policy is applied to healthcare, and in the case of nursing, the government aims to increase the number of nursing graduates. However, it is not clear whether this has been successful. Some issues have been noted in the development of training for native healthcare professionals. A wide range of diploma and degree programmes accredited by British and North American universities and professional bodies were created in response to Saudization policies. However, students were found to experience unexpected difficulties related to limitations in their High School education, including a lack of practical training, a concentration on rote learning, poorly developed study skills, and dependence on theoretical, teacher-centred learning (Al-Sheikh, 1995). In 
addition, it is believed that up to $50 \%$ of nursing graduates are lost each year, some go to work overseas, and many of the men move into managerial positions, increasing reliance on foreign sector workers (Al-Homayan et al., 2013).

Within hospitals, top management is governed by Saudis and the Western workers are responsible for operations and staffing. Western workers are highly concentrated in major hospitals such as NGHA and KFSH\&RC. While essential, the predominance of foreign workers brings with it many issues. Al-homayan \& Islam (2013) look specifically at the issue of nursing in KSA, and concludes that, "the Saudi nursing sector is comprised of nurses from different nations, each one having its culture and traditions which are reflected in the way they deal with patients" (Alhomayan \& Islam 2013, p2586). Language is also a barrier as the nurses communicate with each other in English as a second language, and usually speak very little Arabic which is required to communicate with the patients (Al-Homayan et al., 2013).

All of these issues have a particular impact on the provision of palliative care within KSA and are further explored within the literature review.

\subsection{Motivations for the research}

\subsubsection{About the researcher}

The researcher was born and raised in KSA. However, opportunities for a good education and career in KSA are limited, due to the reasons discussed above, such as the predominance of experienced foreign workers. Thus, having completed my schooling in Saudi Arabia and desiring a bachelor degree I applied to universities in 
Saudi Arabia. However, due to the small number of universities and a large number of students applying, I was not successful in securing a place.

In KSA, important decisions such as education are made with the help of one's family, and my father encouraged me to pursue a career in health, as he had done. The only institute I could attend and stay in KSA was a college that taught a twoyear Nursing Diploma albeit a long way from my home. I applied for a place but unfortunately, again, was not accepted.

My father then suggested that I study in the nearby country of Jordan, and offered to fund these studies. After relocating I applied and was accepted, to do the Bachelor's Degree in Nursing in the capital city, Amman, at the University of Jordan, which is a governmental university. My journey in nursing began here.

The teaching at this University was in English rather than Arabic, my first language. There was no support available for the English language, and often the tutors taught in broken English to large auditoriums of students. It was very difficult in this environment, and I feared I would not be successful. However, by this time I felt entirely committed to pursuing a career in the field of nursing, and I decided to move to another private university which had a reputation for better support, particularly with the English language.

The teaching staff in the new university were all taught in the West. At the beginning, I was worried as this was unfamiliar to me, but I was soon impressed by the standard of teaching in this faculty. The whole teaching team was very supportive, and there were fewer students, which made it easier to seek support 
when needed. In this supportive environment, I scored excellent marks in exams and successfully completely my course. Among the people who supported me was Dr Julia Kilbride from Bradford, UK. She had taught me for some subjects and encouraged me to pursue further studies. At this point, I was particularly interested in palliative care and opted to study for a Master of Advanced Practice (Palliative Care) in Australia. Australia was considered one of the top countries for the provision of palliative care with a high educational standard.

In Australia, with constant practice, I became more fluent in English. This was a wonderful opportunity that enabled me to gain new skills and knowledge in addition to successfully completing my masters in palliative care.

Moving away from my home country gave me the opportunity to study for my career and gain new skills, such as mastering the English language. It also gave me a different world view, and while I always intended to return and work and contribute to the country I was raised in, I was destined to return with a different perspective to when I left.

On my return to Saudi Arabia in 2009 , I worked as a nurse in a medical ward for a year. Following this, I worked in the cancer clinic and then in a palliative care team for six months, after which I worked as a palliative care nurse in home healthcare for two years. To my frustration, I could not transfer and exercise the knowledge and skills that I had acquired abroad due to an apparent lack of understanding by healthcare workers about the importance of palliative care. 
I began to feel and see the turmoil in palliative care in Saudi Arabia. I was asking myself, what are the cultural and professional barriers that prevent the progress of the work of palliative care? It seemed to me that there were professional obstacles, such as lack of employment in the profession and a lack of qualifications, which led to inadequate spiritual care, psychological care and social care. This was in the context of a lack of standards of care and exacerbated by cultural issues such as belief in addiction to pain medication.

There also appeared to be other cultural barriers such as the cultural background of employees being different from the culture of palliative care patients. And other issues, such as families and physicians not telling patients about their diagnosis.

I could see how desperately change was needed, but I reached a point where I was not able to stimulate change within my work. I felt an academic route would allow me to work on changing the culture of palliative care. I gained work as a lecturer in a very new College of Nursing for males, in a city college that provides Bachelor Degrees in nursing. However, palliative care does not exist in its curriculum, so ultimately I was not able to teach very much about palliative care via the subjects I taught.

Finally, I decided the best way to affect change would be to work on research to advance palliative care in Saudi Arabia. This led me to this current PhD study, which will give me the skills to produce work that will benefit the promotion of palliative care in Saudi Arabia. 


\subsubsection{My personal palliative care philosophy}

Based on my journey to date, this researcher believes that palliative care should be provided to all patients who have cancer and non-cancer diseases and should not be decided on the basis of the whether the person is nearing his or her death, but rather it should be decided on the basis of the symptoms and the amount of suffering the patient is going through. It is my opinion that it is important to enhance the level of education and awareness about the need of palliative care amongst the healthcare professionals working in KSA hospitals, a belief which is shared by other researchers in KSA. My position in regards to palliative care has developed primarily from comparing my experiences of palliative care in KSA, with what I have studied in regards to Western healthcare institutions and reviewing the literature of research on palliative care conducted in these institutions. These inputs have made me realize the importance of palliative care in the operation of a successful hospital.

\subsubsection{What is it like to work in healthcare in KSA?}

The government is struggling to provide Saudi people with adequate healthcare due to issues with the quality of staff and the insufficient number of hospitals compared to the population. Other well-known major government hospitals which do not belong to the main health provider, $\mathrm{MOH}$, are NGHA (National Guard Health Affairs) and KFSH\&RC (King Faisal Specialist Hospital and Research Centre). These hospitals are operating to western professional standards and their workers hold accredited certificates, some from developed countries such as the UK, USA, Canada and Australia while others from developing countries. 
Life can be difficult for Saudi healthcare workers. Accommodation is extremely expensive, and public transport does not exist. It is also not safe to walk around the cities. The lack of appropriately qualified staff means that despite a holiday allowance, it is difficult to take holidays because there are not enough qualified staff to cover holiday leave. Similarly, due to lack of appropriately skilled staff, the nurse may be asked to deliver care in an area where they have no specialisation. In some ways, it is better to work within the hospitals that work to Western standards. However, in other ways, this is difficult because they largely treat certain groups, such as employees, members of the Royal Family, National Guard personnel and VIPs, and so working in these places, one becomes aware that the average Saudi does not have equal access to healthcare. There are also issues within the native population of tribalism and racism. This contributes to a corrupt system where promotions are based on who you are, who you are related to, and who you know, rather than merit.

Palliative care is well established in many Western developed countries, such as the United Kingdom (UK). However, in developing and Middle Eastern countries provision is still developing, and is often inadequate (Zeinah et al., 2013). My experience as a nurse working in palliative care in the Kingdom of Saudi Arabia (KSA) was one of frustration at being unable to deliver the appropriate standard of palliative care. The literature suggests that despite some important milestones, such as the opening of a palliative care unit in a major hospital, and the launching of a post-graduate training program (Zeinah et al., 2013), progress has been slow and 
there is a lack of staff who are well educated in palliative care (Al-Shahri et al. 2004).

\subsection{Research in KSA}

KSA has witnessed a rapid development in healthcare, but research outputs fall behind those of Western countries. Research activity in KSA is concentrated within the larger hospitals, due to the availability of funding and the presence of academics from prestigious Western universities, as well as some native staff who have studied abroad. Research has focused largely on medical topics with a lack of attention to other topics such as nursing, or health care education. For example, many funding bodies, including some academic institutions, have been reluctant to give grants for research in medical education. In addition, biomedical companies who often fund research have little commercial interest in medical education (Abdulrahman, 2012).Other research activities take place in the higher education institutes, but the outputs are often of low quality, due to inadequate education in research, and unsophisticated use of Arabic and English. Writing skills in medical education may restrict some medical teachers in publishing their work (Abdulrahman, 2012). The limited numbers of peer-reviewed journals in medical education in both Arabic and English is another limiting factor for publication.

Research into palliative care in KSA is almost entirely focused on cancer care, a factor that is impacted further by inadequate resources given in general to research in KSA. There appear to be a variety of reasons for this, including lack of funding on many levels, lack of interest and awareness of the value of research, lack of 
information from organizations, and institutions and individuals who cite issues with confidentiality, privacy and security as grounds for limiting research. Further, there is a lack of visibility in the media on the most prominent researchers and their scientific productions.

To be able to compete with developed countries in the field of research science, the government of KSA must strive to create centres for research as well as supporting national efforts to develop research. The researchers must also be supported financially, morally, and culturally.

As a conservative Islamic country, it can be difficult for outsiders to access the views of those who work there, and there is a shortage of native people with the skills to carry out research. With the KSA research focus to date having been so heavily in favour of medical research, qualitative research, in particular, has been limited which may help to explain why there is a dearth of information on palliative care issues. Therefore, this researcher determined that a qualitative study designed to ascertain the views of those working with terminally ill patients in KSA hospitals would be of great value, and may help to elucidate the reasons for the slow development of palliative care in KSA. 


\subsection{Vision of Palliative Care in the KSA}

The researcher's vision of palliative care in the KSA is centred on three pillars, which are awareness, competitiveness, and sustainability. First, he envisions a paradigm shift in palliative care in the KSA where healthcare professionals across different levels in the country are aware of what palliative care is and understand the value of this aspect of care to their patients. In this vision of palliative care, healthcare professionals remain focused on curative and preventive aspects of their work, but recognize the third, equally important dimension of palliative care; they are sensitive to the patient's perspective about how the patient is feeling, and know the value of this perspective in determining the best ways to provide adequate care to the patient. Second, he envisions palliative care in Saudi Arabia to be highly competitive in comparison to palliative care in other developed countries. As discussed in the previous sections, while the KSA has enjoyed considerable economic development, many aspects of its healthcare system, palliative care included, have not developed at the same pace. The researcher envisions hospitals in the KSA being staffed with healthcare professionals who have undergone sufficient training on palliative care and are therefore sufficiently competent to address the palliative care needs of their patients. Finally, he envisions palliative care in the KSA to be sustainable. This sustainability is established through the presence of extensive mentoring programs where healthcare professionals who have been properly trained in palliative care can share their knowledge with other healthcare professionals in the country. The need to continuously hire professionals from outside the country in order to fill specialized needs like palliative care is 
diminished since local professionals can already be properly trained and conditioned to fill such needs. Through this process, both competence and value of palliative care may be maintained from one generation of healthcare professionals in the country to the next.

\subsection{Ethical concerns}

Undertaking research in any place requires serious consideration of the ethical implications of the research. In a country such as KSA, the issues to be considered are very different to those in Western countries such as the UK. Not only does the native population have different cultural norms which need to be respected, but the attitude of even well-educated people towards research is different. KSA is not a country which espouses the idea of freedom of expression. People who have been raised in KSA, and also people who work within organisations in KSA, tend to be guarded and suspicious about sharing information. This impacts upon the process of research. However, it also has implication for the value of the research. Reputation is one form of currency in KSA. Because of the researchers own and the researchers family's reputation within healthcare in KSA, access albeit with great difficulty was gained to the views of healthcare professionals in regards to palliative care, and through this insight to increased understanding of the issue of palliative care in KSA.

\subsection{Summary}

This chapter has summarised the aims of the research and provided background information on the country of KSA, its healthcare system, and the challenges of 
working and conducting research in KSA. The cultural environment of KSA has also been described.

Based on this it is evident that Saudi Arabia faces many challenges in providing healthcare to its people, including a lack of healthcare skills in the native population, and the difficulties of providing adequate healthcare beyond the major cities. This chapter provided essential context within which to view this research into palliative care, its aims, design and findings.

An overview of the research environment in KSA has also been provided to help the reader to understand the unique nature of this research and its potential importance. This will be further elaborated in the literature review.

Finally, ethical considerations for the research were provided. 


\section{Literature Review}

\subsection{Introduction}

This study investigated the extent to which the idea of palliative care and palliative care nursing has been accepted among healthcare professionals in KSA. That is, this study determines whether physicians, nurses and healthcare administrators in KSA are aware of palliative care, engage in palliative care practice, and recognise the value of palliative care and palliative care nurses.

Definitions of palliative care will firstly be discussed. Specifically, the World Health Organisation definition and the philosophy this research is based upon. It will then be important to specifically state the definition of palliative care that will be used for the purpose of this study. This chapter will briefly address the origins and development of the modern concept of palliative care, in Europe and other developed countries, developing countries and finally Middle Eastern countries. A comprehensive review of palliative care literature within KSA will be undertaken, and this will be briefly compared to current provisions in the UK. Finally, key issues will be discussed in more detail concerning international literature.

\subsection{Literature review search strategy}

The literature review was performed by searching the following electronic databases: CINAHL, MEDLINE, Academic Search Complete, ProQuest Health \& Medical Complete, ProQuest Research Library, and Academic OneFile. The search terms in Table I were entered separately using the "and" option in the search page of the database, or using the Boolean operator "and" depending on the 
style of the search engine. The following table shows the search terms used and papers found:

Table 1 - Literature review search

\begin{tabular}{|l|c|c|c|}
\hline Search terms & $\begin{array}{l}\text { Total papers } \\
\text { found }\end{array}$ & $\begin{array}{l}\text { Titles selected } \\
\text { for abstract } \\
\text { review }\end{array}$ & $\begin{array}{l}\text { Abstracts selected for } \\
\text { full paper review }\end{array}$ \\
\hline $\begin{array}{l}\text { "Palliative care" } \\
\text { and "culture" }\end{array}$ & 1133 & 59 & 24 \\
\hline $\begin{array}{l}\text { "Palliative care" } \\
\text { and "Islam" }\end{array}$ & 64 & 17 & 3 \\
\hline $\begin{array}{l}\text { "Palliative care" } \\
\text { and "Muslim" }\end{array}$ & 28 & 8 & 34 \\
\hline $\begin{array}{l}\text { "Palliative care" } \\
\text { and "KSA" }\end{array}$ & 372 & 77 & 21 \\
\hline $\begin{array}{l}\text { "Palliative care" } \\
\text { and "spirituality" }\end{array}$ & 1166 & 40 & 29 \\
\hline $\begin{array}{l}\text { "Palliative care" } \\
\text { and "religion" }\end{array}$ & 751 & 94 & \\
\hline
\end{tabular}

Each search generated a list of titles, which were scrutinised to identify potentially relevant papers. Abstracts were then obtained for all papers which may have been relevant judging from the title. Abstracts were then read, papers that were clearly irrelevant were eliminated, and full texts sought for the remainder. At this point, judgement was used to decide whether the full-text paper was to be included in the literature review.

For the literature related to palliative care in Saudi Arabia, no time limit was placed on the search, and all literature about this topic was included, regardless of quality. This was to present a complete picture of the current research landscape. Within the write-up of this section, information is provided on the quality of the sources used. 
The other sections of the literature review can be seen as providing background and context for the main section on palliative care in Saudi Arabia. Given this aim, and the volume of literature available on palliative care in general, not all relevant literature was included. Rather, a sample of the literature which represented the salient issues is presented. The following inclusion criteria were used. First, only papers that were found to have some relevance to different areas of interest in the study based on the specific aims of the thesis were included. This criterion required categorizing each study that was considered under at least one of the aims that were identified in the study. Second, the age of the source was considered in relation to the content that the source offered. Sources that were seminal in nature were included in the study regardless of age, as these informed the background of the study, and no newer sources were found to contain the same relevant information as these. This included such aspects as the history of palliative care in the United Kingdom as the development of healthcare in Saudi Arabia. On the other hand, more recent studies were included when it came to examining areas of interest that are known to change considerably over time, such as best practices in palliative care. In these areas, it was important for the study to focus on current literature in order to be able to accurately contextualize what current practice of palliative care should be like in the intended study setting. With regard to exclusion criteria, papers that were initially included in the review based on the inclusion criteria were excluded based on the quality of the paper. Quality was gauged by the credentials of the author, whether or not the journal where the article is published is peer-reviewed, and the actual content of the paper. Articles that did not present 
a clear methodology or made conclusions that were contrary to what were presented in its results were excluded.

Google Scholar was used to search for general articles on palliative care, which formed the basis for the sections on the history and development of palliative care, and current palliative care in the UK. The search was limited to 2000-2015, and the most recent papers were viewed first. Papers prior to this period were sourced if referred to by a more recent paper and believed to be significant. Other older papers considered seminal texts were used on recommendation from supervisors or other experts.

Other sources of literature were: hand searching of bibliography of highly relevant papers, searching for key authors within the area, and other papers known about through word of mouth or attendance at seminars and conferences.

\subsection{Definitions and history}

\subsubsection{Policy and definitions of and within palliative care}

Traditionally, the term palliative care has been seen associated with care of the dying. However, it has also been used interchangeably with the terms 'supportive care' - that which helps the patient and their family cope with their condition and its treatment (NICE, 2004), and 'terminal care' - the management of care for a patient from the point at which death is expected, whether that be days, weeks or months away (Clark and Seymour, 1999). The term 'end of life care' is used by the National Council for Palliative Care to refer to people who are considered likely to die within the next 12 months (National Council for Palliative Care (UK) 2011). 
The definition of palliative care has broadened, as it has been recognised that many aspects of palliative care need to be initiated as early as possible in an illness such as cancer, in order to support the patient in the best way possible. Therefore, palliative care has become equivalent to supportive care, in that it may be initiated at a stage of illness when death is not certain, along with curative treatments (Mir, 2011). Terminal care has now become broadly equivalent to 'end of life care', but still forms part of palliative care (Clark and Seymour, 1999).

The World Health Organisation have been promoting pain relief and palliative care since the early 1980's (Wright et al., 2008), and recommend that each country has a national policy for implementing palliative care (Webster et al., 2007). Currently, WHO define palliative care as "an approach that improves the quality of life of patients and their families facing the problems associated with life-threatening illness, through the prevention and relief of suffering by means of early identification and impeccable assessment and treatment of pain and other problems, physical, psychosocial and spiritual"(World Health Organisation 2015, paragraph 1).

Palliative care should include relief from pain and other symptoms, in combination with therapies to prolong life if relevant. It should intend neither to hasten nor postpone death, and regard dying as a normal process. It should integrate the psychological needs of both the patient and family, and help the patient live as actively as possible until death, with an emphasis on quality of life (World Health Organisation, 2015). In this and other definitions of palliative care, we see the emphasis on a holistic approach, quality of life, and the inclusion of the needs of 
the family as a whole (Amoah, 2011). Pain control is often the primary concern of palliative care, as without effective pain control, other goals of palliative care may not be achieved. Physical symptoms have an impact on psychological symptoms such as stress and anxiety, with severe pain being associated with suicide (Vachon et al., 1995). Sometimes to alleviate the patients' pain, opioids need to be given in doses so large that they may hasten death. However, the primary intention is to relieve the patient of suffering. Sedation may also be a treatment option in extreme cases (Choong, 2015).

Palliative care is seen as requiring a holistic approach. Holistic care implies that the health professionals perceive the integrated physical, social, psychological, occupational and spiritual needs of the patient, and the National Institute for Health and Care Excellence (NICE 2004, cited in Amoah 2011) suggests ongoing holistic assessment of all these domains throughout the illness trajectory. The WHO places emphasis on quality of life in palliative care. This concept is difficult to define, as it varies between individuals, and in the case of palliative care will also vary throughout the progression of illness (Jocham et al., 2006). It can be seen as related to perceptions of meaning and searching for the meaning in events in one's life (Brown 1963, cited in Amoah, 2011). Some writers have pointed out that quality of life is a factor in all medical specialities, as they all operate on the principle of enhancing people's well-being, and is not exclusive to palliative care (Amoah, 2011).

Another important element of palliative care in the West is 'advance care planning', which is a process of discussion between patients and their healthcare providers, 
regarding plans for future care, when the patient may no longer be able to state their preferences (Amoah, 2011). This can take the form of living wills, which are a person's written records of healthcare preferences, and durable power of attorney (USA) which provides another person with the power to make healthcare decisions on behalf of the patient should they become unable (Garrido et al., 2012).

One of the crucial components of palliative care is to reduce suffering and increase the quality of life (Schultz et al., 2012). While it is possible to define good palliative care, Webster et al., (2007) state that it is "more important to recognise that effective palliative care needs to be appropriate to a particular country's culture, resources and existing health programs" (p30).

\subsubsection{History and development}

The roots of palliative care are in Europe, and the UK are both religious and philosophical, with the first hospice in the form that we currently understand the term, being opened in 1967 by Dame Cicely Saunders in London. However, this can be seen as a key moment in a much longer development of the concept of palliative care. Milligan \& Potts (2009), in a chapter detailing the history of palliative care, within a book concerning palliative care in nursing, explain that the word 'palliative' derives from the Latin 'pallium', meaning cloak or covering, and 'hospice', originally referred to a resting place for pilgrims. The first hospice for the dying is thought to have been in France, in 1842 (Milligan and Potts, 2009). The end of the $19^{\text {th }}$ century saw a rapid increase in medical technology, and it was finally possible to cure major diseases. This pre-occupation with curing illness may have obscured the need to provide a supportive and caring environment for the dying (Gray et al. 1995). In the 
UK, it was religious orders that filled the space left by the medical professionals (Milligan and Potts, 2009).

Today the need for palliative care is growing as the number of elderly people, and consequently, the number of seriously ill people, grows (Malloy et al., 2014). Worldwide, 58 million people die annually with around 45 million of these in developing countries, and $60 \%$ of these will be suffering from advanced illness and in need of palliative care (Malloy et al., 2014). In 1995, the European Association for Palliative Care declared that every individual had the right to pain relief and that palliative care must be provided according to the principle of equity, irrespective of race, gender, social status, national origin, or the ability to pay for services. These recommendations formed what became known as the Barcelona Declaration $(\mathrm{MOH}$ Catalonia, 1995). In many poorer European countries, this is still a goal, rather than reality (Lynch et al., 2009). However, in the UK, for example, since becoming established as a speciality in 1987, palliative care has become very well established with networks of day centres and hospices serving palliative care needs. In 2008, the Hospice and Palliative Care Directory listed 223 inpatient units, 304 hospital support teams, 283 day care services and 316 community services (Milligan and Potts, 2009).

A recent paper in published in a peer-reviewed journal by Choong (2015), discusses the role of Cicely Saunders in the development of the hospice movement. Cicely Saunders had observed hospices run by nuns, which provided comfort for the dying but were not medically well informed or equipped, and wanted to open a modern 
version of the hospice (Choong, 2015). Saunders was influenced by her Christianity, which included the belief in the inherent value of life, and that death was not the end, but a spiritual event (Choong, 2015). The work of the hospices was underpinned by a belief about the inherent value of life, fundamentally opposed to that of the euthanasia movement, and an alternative response to the issue of terminal illness (Choong, 2015).

There was also a recognition of the impact of dying in the wider family, and the family were included within the remit of palliative care (Choong, 2015). A brief biography within a blog on Glasgow University's End of Life Studies web page discusses Cicely Saunders role in developing a holistic understanding of palliative care, through her concept of 'total pain', which she used to explore and expound the relationship between physical and mental suffering. 'Total pain', includes physical symptoms, mental distress, social problems and emotional difficulties. Saunders acknowledged the spiritual suffering of the patient in relation to physical pain and encouraged palliative care practitioners to listen to the patient's story and understand the experience of suffering in a rounded way (Clark, 2004). This concept is reflected in the holistic nature of palliative care. In an article reflecting on the pioneering days of palliative care, when she worked with Dame Cicely Saunders at St Christopher's Hospice, Mary Baines (2010), describes how, in addition to being a key figure in establishing the hospice movement in the UK, Cicely Saunders and colleagues launched the first community palliative care service 'Hospice at Home' in 1969. This was in response to the wishes of patients and based on patients' needs and the wishes of colleagues already working in the community (Baines, 2010). 
From this humble beginning, the idea of Hospice at Home was taken on board and in 2008, 314 such teams were counted in the UK, and its remit has expanded to include diseases other than cancer (Baines, 2010).

St Christopher's Hospice espoused the concept of 'total pain', and it was woven into the fabric of daily life at the hospice and became a defining feature of its philosophy and approach. Cicely Saunders retired from the full-time role of Medical Director at St Christopher's to become its Chairman in 1985, when palliative medicine was just two years away from speciality recognition in the United Kingdom. 'Total pain' can be seen as an innovative concept within the development of palliative care (Clark, 2004).

In their book addressing end of life care globally, Clark \& Centeno (2014) divide the development of palliative care in Europe into three distinct phases. Phase one, 1945-1965, saw the development of ideas about the end of life, in counteraction to the advancements in modern medicine which extended life but at the same time saw death as a 'failure'. During this time, Cicely Saunders led the development of these ideas, introducing the concept of 'total pain' which considered the psychosocial needs of the dying patient, as well as the physical aspects of dying. This may occur when a patient has not obtained closure of certain issues in their life, for example, financial or relationship issues. Intervention may be needed at all levels to ensure a peaceful death. Phase two, 1965-1985, saw the spread of these ideas, and their implementation. These moved beyond recognition of the importance of palliative care and the importance of a patient-centred approach, to a discussion about how this could work in practice, in terms of policy making and 
multi-disciplinary practice. Phase three, from 1985 onwards, has seen palliative care striving towards integration into mainstream medicine.

While there is a lack of research into palliative care in developing countries, many academic writers are drawing attention to the palliative care situation worldwide. Despite the relatively good provision of palliative care in the UK, USA and other developed countries, palliative care can still be seen as an emerging discipline, with the majority of services located in developed countries (Webster et al., 2007). In many countries, there are insufficient resources to provide palliative care, which should involve symptom control and relief from pain and stress (Malloy et al., 2014). Clark \& Centeno (2014) identified a number of factors hindering the development of palliative care globally. These are:

- Lack of education and training programmes,

- Limited awareness and recognition of palliative care needs,

- Lack of information about pain relieving drugs,

- Limited funding,

- Lack of coordination among services and

- Uneven palliative care coverage.

Researchers within the International Observatory on End of Life Care (IOELC), part of Lancaster University, have reviewed developments in palliative care worldwide and categorised palliative care found in each country according to a four-category typology. These stages are no activity, capacity building, localised provision and integration into mainstream medicine. They found countries, including Somalia and 
Yemen, which had no known activity in palliative care. Countries such as Qatar and Turkey were at a capacity building stage, that is, evidence of planning and education, such as attending conferences, was found. In many countries, localised provision was found, including Morocco and KSA. In the UK, USA and other countries, palliative care was approaching integration into mainstream medicine (Wright et al., 2008).

Although pain relief forms only one part of palliative care, it can be seen as the primary concern because if it is not addressed, it is unlikely that psycho-social issues will be able to be addressed. The problems with the provision of pain relief stem from concerns about the misuse of opioids, and the manufacture and availability of opioids (Webster et al., 2007). Some countries also have excessively strict drug laws (Aljawi and Harford, 2012). Education programmes should be aimed at health professionals, informal carers and the general public. For health professionals, palliative care training should be integrated into undergraduate education. Cancer in the developing world is characterised by diagnosis at advanced stages, fewer allocated resources and higher incidence than countries with more developed health systems (Mohammad Z Al-Shahri, 2002). The Middle East Cancer Consortium has been raising awareness about the need for palliative care training at the undergraduate level for the past 15 years (Wright et al., 2008). Within Middle Eastern countries the majority of cancer patients seek medical attention only when cancer has become incurable, and palliative care is the only option. However, it is not always an available option (Zeinah et al., 2013). Within the Middle East, services range from volunteer organisations to official hospital-based units. However, there 
are no standalone units. Palliative care is still not recognised as a speciality and given appropriate funding (Zeinah et al., 2013).

The majority of the population of the Middle East are Muslim, and the Middle East forms a large portion of the Islamic world. Zeinah et al. (2013) state that: "The available healthcare services in the Islamic world clearly do not meet patient's needs, and there is little sign that this will improve in the foreseeable future" (p60). However, he also notes signs of positive improvements, such as the increase in the use of morphine to treat pain in Islamic countries.

\subsection{Prospective Lessons for Palliative Care}

Given the history of palliative care in the United Kingdom, as narrated in the previous section, some important considerations need to be made in examining palliative care in another geographical and socio-cultural context. One important aspect is the difference in religious foundations between the United Kingdom and the KSA. As found from various sources, palliative care in the United Kingdom has been heavily principled in Christian values (Choong, 2015; Milligan \& Potts, 2009; Gray et al. 1995). In contrast to this, the KSA is an Islamic country and therefore may have some principles that different from the Christian context when it comes to supporting palliative care. While it may be argued that there are many similarities between Islam and Christianity, such as the inherent value of life, and the consideration given to the wider family, it is important to explore uniquely Islamic values when it comes to the treatment of individuals at end-of-life in order to determine how the merits of palliative care can appeal to local healthcare providers. While palliative care is needed and applicable worldwide, it may need to 
develop in unique ways to serve patients well where culture is decidedly different from the Western culture. It is also important to learn from the UK experience regarding problems that were encountered in the development of palliative care, and how these same problems may be present in the KSA setting.

\subsection{Palliative Care in KSA}

Palliative care is an area of healthcare which is focused on alleviating and/or preventing the suffering experienced by patients across various conditions and it encompasses the care of patients in different stages of illness, from those who are undergoing curative treatments to those who are terminally ill and nearing the end of life (Becker, 2009). In recent years, palliative care has grown tremendously as an integral part of healthcare institutions worldwide (Hui et al., 2010). However, not all countries have well-established palliative care services or recognise the importance of palliative care (Hui et al., 2010). It is important for healthcare professionals to recognise the value of palliative care, and to be able to provide such care to patients. There may be professional and cultural barriers that prevent practitioners in specific contexts to be able to do so. This is the premise from which the motivation to conduct this study was drawn. Specifically, this study considered the context of KSA, which is known to be culturally different from the West where the concept of palliative care originated and where the recent progress in palliative care is most evident (Hui et al., 2010).

The literature review as a whole does not claim to be exhaustive, however, as the literature on palliative care in KSA is limited, it is possible to be confident that all of the relevant literature has been reviewed. At the same time, it is important to 
consider that the concept of loss is absent in KSA literature. That is, the religious belief that those who die are not truly lost but simply transcended to another state of being is so strong that it prevents academic discussion of the alternative in local academic literature.

The literature discussed below has been summarised in a table and can be found in Appendix 1 (p249).

\subsection{Background to Palliative Care in KSA}

Cancer incidence in KSA is around 38 per 100000 (Almobarak, 2014). Cancer patients make up a large proportion of palliative care patients, and development of palliative care in KSA has largely centred on cancer patients. Most newly diagnosed cancer patients present in later stages of the disease, where palliative care may be more appropriate than curative treatment (Mohammad Z Al-Shahri et al., 2004; Alshammary et al., 2014). While cancer patients may make up the majority of those with a palliative care need, there are other life-threatening illnesses which create a palliative care need. Ghanem, Rawabi, Abou Alia, Al-Zayir, \& Alsirafy, (2011) reported on non-cancer referrals to a palliative care service within a tertiary hospital in the Eastern Province of KSA, and found that $4.2 \%$ of palliative care patients had a non-cancer diagnosis, and were largely referred due to pain. This was a retrospective review of the "palliative care inpatient database", a comprehensive database used by the hospital to record inpatient palliative care. There was no record of outpatient palliative care, and therefore this could not be reported. However, the study refers to a previous study which reports low referral 
rates of non-cancer patients for palliative care (AI-Sirafy 2007, cited in Ghanem et al. 2011). Ghanem et al. (2011) concluded that:

"Although patients with life-threatening illnesses other than cancer have palliative care needs comparable to those of patients with cancer, these needs are largely unmet due to many barriers" (Ghanem et al., 2011).

\subsection{Current palliative care provision}

KSA's first palliative care provision was a two-bed facility at King Faisal Specialist Hospital and Research Centre (KFSH\&RC), opened in 1992. This has developed into a centre comprising an intensive management unit, consultation service, outpatient clinic and home healthcare programs. The hospital also has a home health programme, where palliative care patients within a $50 \mathrm{~km}$ radius of the hospital are visited by health professionals at home (Alsirafy et al., 2009). In 2002, ten years after it first opened, the facility introduced a two-year post-graduate training facility (Zeinah et al., 2013). In 2010 a palliative care unit (PCU) was established within the Comprehensive Cancer Centre, King Fahad Medical City, consisting of 9 inpatient beds, as well as outpatient and home services (Alshammary et al., 2015). Progress has been slow in establishing palliative care more widely, and more than two-thirds of cancer patients in KSA will have limited access to specialist palliative care services (Al-Shahri et al. 2004). There has been no national action to improve palliative care, and aside from what is provided in the primary health-care system, it remains a matter of local provision (Abudari et al., 2013). However, notable developments include the establishment of palliative care provision in Jeddah in 1998 (Zeinah et al., 2013) and the development of a two-bed unit in Riyadh in 2004 
(Alshaikh \& Sormunen, 2015). The initiative at Jeddah is only the second since the one at KFSH\&RC, which means that it took over seven years for palliative care to become a formal concern in another institution in KSA (Zeinah et al., 2013). By 2010, which was the most current data collected by Zeinah et al., (2013), there were already over 15 cancer centres in the country, each with its palliative care system. However, Zeinah et al., (2013) admitted that this progress was insufficient, as the need for palliative care was found to grow with the number of institutions that began offering it. The development of palliative care is hindered by the fact that there are no nurses in the Kingdom who specialise in palliative care, and only a small number of physicians specialising in palliative care.

Al-Shahri et al. (2004) has cited the palliative care provision at KFSH\&RC as advanced compared to other countries within the Eastern Mediterranean Region (EMR) and suggested collaboration between the World Health Organisation and expertise at KFSH\&RC to create a regional centre of excellence for the EMR. This was to include a fellowship programme, and annual conference and the drafting of palliative care guidelines. It is not clear that this initiative ever took place, and there are now arguably other countries in the region with examples of excellence, for example in Kuwait (Kuwait News Agency, 2014).

Alsirafy, Hassan, \& Al-Shahri, (2009), presented various statistics on palliative care in KSA and from a review of 629 medical records at KFSH\&RC, found that $86 \%$ of patients admitted to the palliative care unit (PCU) died in the hospital. This was high compared to PCUs in other countries. The reason behind this higher rate of 
mortality was considered to be the greater use of home care in KSA, which leads to patients being brought to the PCU when their conditions were already at a critical stage. However, hospice care is not available, and palliative care at home services are insufficient (Abudari et al., 2014; Almobarak, 2014). Home based patients are advised to go to the emergency hospital if they become very ill, especially at the weekend. This means that many terminally ill cancer patients die before reaching the hospital, or are hospitalised in a stressful setting, and are likely to die in the hospital after a long stay (Alsirafy et al., 2009).

In addition to this, another factor that contributed to the mortality rate was that many patients, after being admitted to the hospital, are no longer able to be discharged even when they have stabilised and can be transferred back to home or hospice care. Often there is no suitable facility to discharge patients to, and the risks associated with travel are often too great to insist the patients go back after they have received treatment.

In a paper detailing the history of palliative care in KSA, palliative care consultant at KFSH\&RC, Alan Nixon (2003) concluded that the present palliative care model in KFSH\&RC is inadequate in addressing all the unique needs of cancer patients in KSA. Progress within KSA in regards to palliative care appears to be slow, with Almobarak raising similar issues in his paper reviewing the literature regarding cancer care and palliative care in Saudi Arabia, in 2014. The ongoing inadequacies in palliative care lead terminally ill patients to suffer considerably as inpatients in a stressful setting and contribute to the high death rate experienced by the hospital (Almobarak, 
2014). Alsirafy et al., (2009), emphasised the need to examine the present model and make changes to it in accordance with the demands of the Saudi setting. However, specific recommendations of what those changes ought to be were not provided, the study only pointed out that settings used in other countries, as revealed from literature, were found to be considerably better than those experienced at KFSH\&RC.

In 2004, Al-Shahri et al., set out a proposal for a national palliative care programme in KSA, pointing to the unnecessary suffering of patients and their families, and the ethical obligation of health policy makers for the promotion of palliative care in the country. Al-Shahri et al. (2004) point out that the structure of healthcare of KSA, with its well-established referral system, means that establishing palliative care should be practically feasible. They suggest that one or more of the main referral hospitals in each region should be designated as the palliative care centre for that region. However, to date, this system has not been adopted by the Saudi authorities.

\subsection{Evaluation of palliative care in the Middle East}

Ideally, palliative care is integrated into a continuum of care from the diagnosis of illness throughout the progress of the disease, and its role increases with the approach of the end of life. Certain quality markers have been identified which can be used to assist the evaluation of existing palliative care provision. These are: Time between referral and death; Time between last chemotherapy and death; Use of antimicrobials; Terminal hospital length of stay. 
Two studies were found that sought to model palliative care and identify challenges that face palliative care in the context of the Middle East. In one of these studies, Al-Awamer and Downar, (2014) explored the Middle Eastern palliative care service model through interviews with healthcare professionals who have worked in both Middle Eastern and Western settings. The objective of Al-Awamer and Downar, (2014) was to compare the two settings and from this, draw out a working model for palliative care which can guide its development in Middle Eastern countries. Results of Al-Awamer and Downar, (2014) found four differences between Middle Eastern and Western settings. First, there were cultural differences in terms of control over information and decisions for terminal patients. Al-Awamer and Downar, (2014) discovered that in Middle Eastern contexts, the family rather than the individual has greater control over what information should be provided to a terminally-ill patient and how end-of-life care should be carried out. Another difference was the influence of religious teachings on decisions made on end-of-life care. The use of painkillers needed to have permission from religious authorities, which interviewees felt affected family members' decisions on letting doctors and nurses administer such to patients. Differences were also found in Middle Eastern institution's general philosophies about palliative care (Al-Awamer and Downar, 2014). Interviewees believed that Middle Eastern institutions did not prioritize palliative care concerns and some did not feel this to be a necessary element of care. Other challenges that were identified in the study were a shortage of resources, underdeveloped policies and laws, and general unfamiliarity of local professionals in the role and benefits of palliative care. It is important to 
differentiate the work of Al-Awamer and Downar, (2014) from that of this study in that Al-Awamer and Downar, (2014) mainly interviewed healthcare professionals who have had experience in both Western and Middle Eastern settings, whereas this study is focused on the KSA setting and local healthcare professionals working in this setting. While the conclusions drawn by Al-Awamer and Downar, (2014) are informative, they cannot be considered as sufficiently accurate in the context of palliative care practice in the KSA.

The other study that was found used a survey to examine the knowledge and attitudes of nurses working in a hospital in Riyadh about palliative care. Abudari et al., (2014) surveyed almost 400 nurses working in Riyadh and found that nurses had moderate attitudes towards the value of palliative care but had deficient knowledge with respect to both palliative care concepts and practice (Abudari et al., 2014). Abudari et al., (2014) found that the experience and training received by nurses significantly affected their scores, with nurses having a more experience and training getting higher scores. As with Al-Awamer and Downar, (2014), the work of Abudari et al., (2014) considerably informs this study, but leaves room for deeper exploration about the attitudes and perspectives of healthcare professionals working in the KSA setting about palliative care.

\subsubsection{Time between referral and death}

In the case of cancer, both the American Society of Clinical Oncology and recent randomised controlled trials, advocate referral to palliative care at the time of diagnosis (Alshammary et al., 2015). The early referral has been shown to improve the quality of life and even prolong life in some cases (Alshammary et al., 2015). 
Studies reviewed in Alsirafy et al. (2010) have shown that clinicians with good knowledge of palliative care are more likely to make early referrals. Early referral to a palliative care service makes it possible to detect and prevent various aspects of suffering and to improve the quality of life of cancer patients and their families (Alsirafy et al., 2010).

According to Gray, Ezzat, \& Volker (1995), referrals to palliative care in KSA were delayed because local practitioners lacked confidence, knowledge or skills in handling the palliative care needs of their patients. As a result, patients were left unaware of their palliative care options, which resulted in considerable suffering during the end stage of their diseases, and the opportunity to develop a good relationship with palliative care professionals over time was lost. Almuzaini et al., (1998) also suggested that the attitude of Saudi health professionals may be a barrier, as two-thirds of Saudi physicians showed a negative attitude towards working in palliative care, compared to $90 \%$ of non-Saudis who showed a positive attitude.

These studies have been included for their relevance, despite being over twenty years old. Alan J Gray (Gray et al. 1995) was a non-native consultant working in KFSH\&RC, and the study by Almuzaini et al., (1998) was a large-scale study which does not appear to have been replicated since. It can be hoped that the developments in palliative care in SA since 1995 would have led to significant improvements in this situation. However, Al-shahri, Sroor, Alsirafy, \& Med, (2010), describe a tendency of referral teams to delay referral to palliative care until the 
patient presents with a range of complex physical and psychosocial problems. An intervention designed to implement referral criteria was undertaken (Al-Shahri et al., 2010) and this resulted in a more rational pattern of transfer of care, suggesting that there is potential for improving this situation.

Alsirafy is a physician and well-known researcher in the field. Alsirafy et al. (2010), undertook a retrospective review of 1567 referrals to palliative care, to review those who ultimately died in hospital. They concluded that referral of in-hospital cancer deaths to palliative care occurs late and many patients are referred when death is imminent. Patients referred earlier to palliative care have a better survival rate. The time between referral and death is shorter than in other countries when compared to international literature. Research is needed to identify the reasons for this, to put in place interventions which will result in earlier referrals to palliative care. In the context of the results of other studies, we may consider that the patients themselves may present late in the disease trajectory, and so referral practices alone may not fully account for these results. The results may also be affected by not including patients referred to outpatient palliative care services, who tended to be included in other studies (Alsirafy et al., 2010).

At the recently established PCU in King Fahad Medical City, referrals were found to occur late in the disease trajectory, and research by Alshammary et al., (2015) reflected upon the underlying causes of the late referrals. Having concluded that the timing of referrals to the unit was suboptimal, the researchers suggested a variety of causes for this, which reflected those suggested by Gray et al. (1995). Despite significant structural developments in the last twenty years, palliative care 
is relatively new to KSA and is not yet fully accepted and understood by both patients and medical professionals. In addition to this, a large proportion of the Saudi population lives rurally, and lack disease awareness and access to disease management, meaning that they do not come to the attention of medical professionals until relatively late in the disease trajectory. These issues may be exacerbated by the lack of Arabic language within the mainly foreign medical workforce, as discussing such complex issues as those involved in end of life care requires good communication.

In order to improve referral patterns Alshammary et al., (2015) advocated increased resources for palliative care, in addition to education programmes in order to raise awareness in the general public and the healthcare professionals, and funding for research to develop optimal referral criteria.

\subsubsection{Time between last chemotherapy and death (TLCD)}

The time between last chemotherapy and death (TLCD) is used internationally as an indicator of the quality of care at the end of life, with a longer TLCD being associated with better quality of care. Using a retrospective review of adult official medical records, Karim et al. (2015) found that $35 \%$ of patients in KSA had a TLCD of 60 days or less. Karim et al. (2015) found that this $35 \%$ were more likely to die of treatment-related causes, in an Intensive Care Unit, and most significantly, were less likely to have had palliative care involvement. Based on figures which show a lengthening TLCD when palliative care is involved (Karim et al., 2015) this suggests the importance of palliative care involvement as early as possible in the course of treatment. These results reflect similar studies in the Western world, suggesting 
that palliative care is associated with less aggressive forms of treatment and better quality of care at the end of life (Karim et al., 2015). A limitation which the authors acknowledge was possible selection bias caused by lack of outpatient data.

\subsubsection{Use of antimicrobials}

Another issue in relation to medication is the use of antimicrobials for palliative care patients during the last week of life. Given the aims of palliative care to improve the quality of life and relieve suffering, antimicrobials in the last week of life are seen as an aggressive and futile intervention. A study by Al-Shaqi et al. (2012)in KFSH\&RC was conducted which found that antimicrobials were being used too liberally with patients approaching death.

\subsubsection{Terminal hospital length of stay}

Terminal hospital length of stay (LoS) may also be used to assess the quality of palliative care, as a longer hospital stay is considered to be associated with aggressiveness of end of life care (Alsirafy et al., 2013). In KSA, due to the absence of sub-acute models of care, such as palliative care consultation (PCC), many patients are looked after within inpatient palliative care units (PCUs). In a largescale study, Alsirafy et al. (2013), looked at the length of stay within a PCU at King Fahad Specialist Hospital, Dammam. A PCC was also available. Patients for whom the care was transferred to the PC service earlier before the terminal hospitalization (PCU group) had significantly shorter LoS. This may add to the current evidence suggesting that earlier PC intervention reduces the aggressiveness of end of life (EoL) care. 


\subsection{Summary of quality indicator evidence}

The quality markers discussed above in relation to KSA, indicate the improvements are needed to decrease aggressiveness in end of life care. A review of hospital records in a tertiary care hospital by Al-Zahrani et al.(2012) looked at overall aggressiveness in end of life care. In this study, results demonstrated that a significant proportion of cancer patients receive aggressive care at the end of life. The authors recommend establishing an integrative palliative care program to improve the quality-of-life of dying cancer patients in the region and to minimize the aggressiveness of end of life care.

\subsection{The role of nursing}

Nursing is central to palliative care, and insufficient knowledge of palliative care among nurses is a barrier to quality provision (Abudari et al., 2014). Education about palliative care in the nursing curriculum is thought to be a worldwide problem, but educational interventions have been found to be effective in improving attitudes towards palliative care (Abudari et al., 2014).

Youssef et al., (2015) attempted to provide a comprehensive picture of the issues around palliative care education in KSA, by evaluating intensive care nurses' attitudes to and experience of palliative care, and also evaluating the palliative care content of the undergraduate nursing programme, using questionnaires developed by the researchers. More than half of the nurses were found to have poor knowledge of, but a positive attitude to, palliative care. He also found that the content of palliative care education was felt to be inadequate by teaching members. Youssef et al., (2015) suggest that attention should be given to palliative 
care in both education and policy. The quality of this research is brought into question by its publication in an open access journal of dubious quality. None of the members of its editorial board was regarded as an expert in the field of nursing or palliative care. Moreover, the relevance of this study is limited, as most nurses are expatriates. Therefore knowledge about palliative care in nurses will depend on their country of origin and may vary widely (Abudari et al., 2014). In a study highly relevant to the current study, Abudari et al., (2014) set out to evaluate knowledge and attitudes towards palliative care in nurses in KSA. This study used pre-defined instruments to quantify the nurse's knowledge and attitudes and test a correlation between these. The chosen instruments were designed to measure knowledge of palliative care and attitudes towards the dying. The study also looked at the correlation between these scores, and the demographics of the nurses, including their place of origin. The results suggested that in KSA's varied expatriate nurse workforce, there was a large variance in palliative care education depending on the country of origin. Their knowledge and attitudes were also influenced by the degree of integration of palliative care into the health-care system, and educational opportunities post-qualification. There was an expected correlation between knowledge and attitudes. Most of the nurses in the study were from countries with the isolated provision of palliative care. The nurses scored relatively low on the measurement instruments, but this does not necessarily reflect the lack of palliative care education in KSA as such and in fact, shows the study is not looking at palliative care education in KSA, rather, it is assessing the knowledge of nurses working in KSA, as only 32 nurses in the study 
were native to KSA. Nurses from countries with advanced palliative care provision scored highly on the measurements. Nurses who had had training in palliative care also scored higher, demonstrating the impact of palliative care education and training (Abudari et al., 2014).

Due to the varied backgrounds of nurses working in KSA, palliative care education should be integrated into KSA hospital systems, and conducting specific training for a core group of nurses in each speciality (Abudari et al., 2014). These findings agree with the observations of writers on international palliative care, such as Nyatanga (2002) and Mir (2011) who suggest care providers from the same cultural background will be able to provide the most appropriate care, and in the absence of this, care providers should have a level of cultural and religious awareness. Similarly, Alshaikh \& Sormunen (2015) explored nurses knowledge of palliative care using a qualitative methodology, within intensive care units in KSA. While the sample size was small, the results support and enhance the findings of Abudari et al., (2014). The study found that nurses lack knowledge about applying palliative care, particularly emotional care and pain management. However, nurses provided good physical care, and the authors also suggest that they provided good spiritual support. They had limited roles in the ICU due to department policies which prevented them from administering medications, making decisions and communicating with families. Nurses also faced challenges in communicating with patients and their families due to language barriers (Alshaikh \& Sormunen, 2015). 


\subsection{Access to pain medication}

Pain is a human experience that impacts upon quality of life, and pain management is a significant element of palliative care. It affects $20 \%$ to $50 \%$ of cancer patients at the time of initial diagnosis and active treatment, and $55 \%$ to $95 \%$ of those with advanced cancer (Al-Rowaili et al., 2009). Studies suggest that by following simple clinical guidelines, such as those of the World Health Organisation (WHO 1986, cited in Al-Rowaili et al. 2009), pain can be effectively managed in $70-97 \%$ of patients with advanced cancer (Al-Rowaili et al., 2009). The mismanagement of pain has implications for human suffering, and an economic impact (Albaqawi, 2015). A study by (Al-Zahrani et al., 2014) set out to determine the prevalence and severity of cancer pain among new and follow-up patients attending a PC outpatient clinic in KSA and asked patients to score the severity of their pain between 0 to 10 . Of those, $85.5 \%$ reported pain, with a median intensity score of five. This was compared with an international literature review which put the prevalence of pain in advanced cancer patients at $65 \%$ (Beuken et al., 2007). The study by Al-zahrani et al. (2014) excluded patients who were not deemed mentally competent (defined as oriented in time, place and person). This was clearly necessary for them to be capable of scoring their pain. However, it may have excluded patients suffering from more severe pain which would affect their mental capacity.

Almobarak (2014) suggests that as many cancer cases are identified at a late stage when pain is already a significant issue, effective palliative care is even more essential. This may be hindered by the country's highly restrictive policies on pain medication. As found by Al-Shahri (2009) the per capita consumption of morphine 
in the country is less than $0.35 \mathrm{mg}$, which when compared to the worldwide consumption of $5.6 \mathrm{mg}$ shows that people in KSA are provided with much lower doses of pain medication than people in other countries. This is concurred by Alshammary et al. (2014), suggesting that this problem is ongoing. It is clear that KSA citizens have very limited access to pain medication, which has critical ramifications for the quality of palliative care in the country. Further evidence is provided by Al-Rowaili et al. (2009), who undertook a large-scale study in a tertiary hospital in KSA, to assess the effectiveness of pain management. Using patient interviews and a pain scale rating, observing practice and reviewing medical records, the study found that $40 \%$ of patients experiencing pain were not given any pain medication and only $60 \%$ were receiving pain management.

Alshammary et al., (2014) suggest that the reasons for inadequate pain management include the lack of training and confidence of healthcare staff in using such medication, the fact that family physicians cannot prescribe such medication, and public misconceptions about the use of opioids for pain relief.

Another possible area of difficulty is suggested by Albaqawi (2014), who claims that pain management can become more complicated in situations where there are cultural differences between nurses and patients.

While palliative care is certainly not just about pain management, and pain management, in turn, is not just about administering pain medication to patients, pain medication is nonetheless a critical component of palliative care. The World Health Organisation (2002) states: "A palliative care programme cannot exist unless 
it is based on a rational drug policy including...ready access of suffering patients to opioids" (cited in Aljawi \& Harford, 2012 :1).

Pain medication serves the role of directly alleviating physiologically caused pain so that other palliative care strategies can be used to treat emotional and psychological pain that are felt by the patient. Pain medication, therefore, serves as the first line of defence of palliative care professionals. When there is insufficient pain medication available, it can be expected that the initial needs of palliative care patients could not be met, which would make it more difficult for practitioners to address the other issues concerning their patients. This places palliative care practitioners at a significant disadvantage in attempting to help patients deal with the pain that they are suffering.

This has been a consistent problem, as it was also mentioned in the work of Gray et al., (1995) 20 years ago. According to Gray et al. (1995), the reluctance of doctors to administer morphine may be due to culture-affected misinformation, where doctors may look at the substance as a tool for euthanasia, an act that is perceived as immoral in KSA. Doctors may feel that by allowing patients to have greater access to morphine, they are encouraging them to use the substance more often and in doing so, are contributing to the possibility that they would die sooner than they would have without such open access to pain medication. According to their culture, this would place them in the position of being responsible for the untimely death of their patients. Similarly, doctors may also feel that giving patients greater access to morphine can increase the chance of such patients becoming addicted to pain medication, which is another cultural taboo in KSA. A report of a symposium 
workshop on the distribution of narcotics at a cancer conference in KSA (Isbister and Bonifant, 2001) cited many of the same issues, and suggested the introduction of the World Health Organisation 'analgesic ladder'. This is a process of stepping up medication appropriately, including the transition from non-opioid to opioid pain medications, as the disease and pain progress (WHO 1986). However, the desired progress does not appear to have been made, as the misconceptions about addiction to pain medication were also mentioned in the research more recently (Zeinah et al., 2013). When in fact, properly administered pain medication has a negligible risk of making a patient addicted.

Pain was also mentioned as a significant factor in a cross-sectional descriptive study published by (Ibrahim et al., 2013). It was recommended that the oncology pharmacist is included in the patient support team, to improve pain management. As explained by Zeinah et al. (2013), and consistent with Al-Shahri (2009) the reluctance of practitioners to provide ample pain medication can be one of the misconceptions that doctors in KSA are prone to having which prevent them from being able to deliver quality palliative care. This suggestion is based on observation, and there does not appear to be any research which supports this suggestion. However, Beuken-van et al., (2007) believe that these issues are less likely to contribute to poor palliative care outcomes, than the lack of palliative care services in most hospitals. Al-Shahri (2009), disregarded culture as the cause for this suboptimal use of pain medication since the $\mathrm{MOH}$ allowed the use of opioids and Islamic writing has little to say against the medical use of such substances. Instead, he argued that the problem may lie in practitioners' knowledge and competence in 
their role as pain managers. This opinion is supported by the results of a study by Alamri (2012). A questionnaire was used to assess knowledge of resident physicians at a university hospital in KSA, on palliative care. The majority of respondents were Saudi Nationals. The study concluded that the participants had suboptimal knowledge of basic palliative care (Alamri, 2012).

A recent thesis by Albaqawi (2014) aimed to explore nurses knowledge and attitudes towards pain relief, in Hail region hospitals in KSA, using a mixed methods approach. The study found that in addition to a lack of knowledge about pain medication, nurses faced such barriers as language, and religious and cultural factors when assessing pain.

Alshammary et al., (2014) suggests a three-dimensional approach to improving the availability of pain medication in KSA. This would include education to healthcare providers about the safe and appropriate use of opioids within palliative care, expanding palliative care services to secondary level hospitals, making the necessary medications available from these services, and increasing availability in the market.

Difficulties with access to appropriate pain medicine in KSA are well documented throughout the literature, and although many pleas for change have been issued, the continuation of these reports suggests that the problem has not been solved as yet. This, is despite the apparent acceptance of the need for appropriate pain relief: 
"The goal of allowing patients with advanced cancer to die with dignity and without pain was identified as a worthy one and one that would be appreciated not only by patients themselves but by their relatives, carers, religious leaders and government" (Isbister \& Bonifan, 2001 p140).

\subsection{Non-pain issues}

Due to the importance of pain management in palliative care as the basis for providing effective palliative care, and the difficulties in providing adequate pain relief in KSA, much research has focused on this area. However, a study conducted by Al-Shahri, Eldali, \& Al-Zahrani, (2012) aimed to explore the prevalence and severity of pain and non-pain symptoms in cancer patients attending a palliative care outpatient clinic. Over a five-month period, consecutive adult cancer patients attending PC outpatient clinic at a tertiary hospital were evaluated for the presence and severity of ten non-pain symptoms, using a numerical scoring system. The most common symptoms found were tiredness, dry mouth, anxiety and depression. The limitations of the study included a small sample size and a limited list of symptoms. However, drawing on the results, the authors emphasise the need for comprehensive assessment and symptom management plans to improve the quality of life of patients.

Another important element of palliative care is 'do not resuscitate' (DNR), practices at the end of life. A study at King Abdul Aziz Medical City, one of the few hospitals in KSA with an official DNR policy, aimed to determine compliance in the documentation of DNR procedures (Gouda et al., 2010). Documentation was 
examined over a three-month period and found that compliance with policy was poor, including addressing the DNR status of the patient on admission, completion of DNR documentation and explanation of the decision to patient or family. This implies that DNR is not always applied appropriately. Religious, cultural and educational issues may play a part in this (Gouda et al., 2010). Due to the cultural norms of KSA, DNR is never discussed directly with the patient, but with the family. Gouda et al. (2010) state that:

"the global medical community must educate and help patients and families to realise that there frequently comes a point when best medical care will not result in survival and will only prolong the suffering of patients and their loved ones" (p2152).

\subsection{Legal issues}

Aside from cultural and professional peculiarities in the KSA palliative care setting, legal characteristics have also been explored. Specifically, Babgi (2009) compared and contrasted legal issues in palliative medicine in KSA and the US. The study examined laws and compared cases between the two countries and found that there existed both important convergences and divergences that have implications to the end of life palliative care offered in KSA. While Arabic law is based on Islamic tenets and US law is based on an entirely secular system, both uphold the importance of life and the need to preserve it. However, at the same time, laws in the US put as much importance on individual rights and the need for people to be as comfortable as possible during the last of their days. As such, healthcare facilities 
in the US are more likely to consider patients' wishes and to monitor how a patient is feeling more closely, whereas facilities in KSA tend to see these responsibilities as falling more upon the family members of the patient than the practitioners in the facility itself.

The emphasis of the end of life care in KSA is on preparing the patient to rest and go back to Allah, whereas facilities in the US. have been mandated to have considerable responsibility in making sure that a patient does not suffer needlessly under their care (Babgi, 2009). As such, the study was able to show that legal directives on palliative care are present in the US that are not yet present in the context of KSA. Part of the reason behind this is the difference between how the two cultures perceived ownership of life. Whereas the US places this ownership upon the individual, KSA culture perceives ownership of life as Allah's alone. As such, whereas the healthcare system in the US can be seen as directly serving the interests of the patient, that in KSA can be seen as serving the interests of Allah as expressed in Islamic laws, which means that protocols cannot be changed because of individual patients' preferences. An obvious consequence of this where palliative care is concerned is that saving and prolonging life is of higher priority in KSA hospitals than making the patient comfortable and following the patient's wishes where their need for comfort is concerned, such as in the case of withdrawal of life support (Babgi, 2009). In such cases, the protocol of protecting the value and sanctity of life would take precedence over the needs and preferences of the patient. 
While doctors are supposed to care for their patients above all else, this directive is overridden by the need for all people in the country to protect the cultural norms that define their society. As such, doctors will not provide patients with the means through which they can end their lives even if such patients already desire to die. In the context of this study, protocols in palliative care typically entail providing the patient with pain-killing medication.

The issues arise when such medication may affect the remaining lifespan of the patient. In such situations, doctors would have to make a choice between allowing the patient to use pain medication at risk of reducing their lifespan or withholding such medications from them with the purpose of enabling them to live longer although in great discomfort.

\subsection{Cultural and religious issues}

According to the observations of Derek Younge (1997) medical professor and surgeon at KFSH\&RC, certain aspects of the culture of KSA impact upon aspects of the palliative care process. This can be challenging for health professionals who are unfamiliar with the culture, but Al-Shahri (2002) suggest that by educating these health professionals in Saudi culture, many difficulties can be overcome. The Islamic belief system will have implications for the way illness is handled, and the way care is received. Islam is not a religion in which worship is compartmentalised and separate from other aspects of life, so it tends to pervade all life issues. Therefore, it will have a significant impact on health beliefs and the experience of death for Muslims, and health professionals need awareness to understand and assist Muslim patients: 
"Improvement in the awareness of healthcare professionals about Islamic teachings is likely to promote sensitive caring for their Muslim patients"(Al-Shahri \& AlKhenaizan, 2005, p435).

\subsection{Islamic perspective on illness and death}

The belief system of palliative care patients native to KSA is Islam, and these beliefs will have an impact on how the patient copes with aspects of their illness. From an Islamic perspective, illness and difficulty are perceived not as a punishment but as an atonement for one's sins (Mohammad Zafir Al-Shahri, 2002). A study by Babgi, (2010) of pain coping behaviours of Saudi patients, found that they employed both prayer and Quran reading, and other religious practices, in coping with pain.

Almuzaini et al., (1998) in a study of the attitudes of health professionals, cancer patients and carers in KSA, found that despite $67 \%$ of patients in his study being at an advanced stage of cancer, $90 \%$ of them rated their health status as satisfactory or good. This optimism was summarised by many in the phrase "Alhamdulillah wa shukro lillah" (Praise and thanks be to God) a phrase used to indicate acceptance of their state as an expression of God's will. Almuzaini et al., (1998) states that the Muslim perception of death as an event which returns him to his Creator, and the pain and suffering is a test of faith. This and the strong family bonds mean that the people of KSA are arguably better equipped than elsewhere in the world to deal with the stress of cancer.

\subsection{Religious practices}

The rites of Islam will remain of utmost importance to most Saudi patients, whatever stage of illness they are at. There are several factors that, despite the 
individual differences between members of the Islamic faith, should be taken into account to ensure the comfort of Muslim patients. The patient's clothes and body should be free from urine, stool, blood or vomit, to enable the patient to be considered ritually clean and therefore able to perform prayers (Al-Shahri \& AlKhenaizan, 2005). They may need support to fulfil the obligation of prayer and may want to fast during Ramadan even against medical advice (Mohammad Zafir AlShahri, 2002). Saudi patients may seek spiritual healing alongside medical treatment. Al-Shahri (2002) recommends that health professionals express respect for these cultural practices, to maintain a good relationship with the patient and family.

In the time before death, a peaceful environment in which Quran can be recited will be appreciated by family members, and after death, a speedy documentation process to allow the family to begin the rituals of washing and shrouding the body. The death rituals for Muslims form the final bonds between the deceased and the bereaved (Gatrad and Sheikh, 2002). During the last moments of life, those accompanying the dying will encourage them to say the Shahada (testimony of faith). Death in a hospital setting is not in keeping with Islamic tradition. The wider family and community will expect and be expected to visit and may wish to stay to recite prayers and Quran, which can be difficult within a hospital setting (Gatrad and Sheikh, 2002). The body of the deceased in Islam is handled in a gentle and dignified manner. The washing, shrouding, and burial of the body should occur as soon as possible after death (Al-Shahri 2005). 


\subsection{The family and 'truth-telling'}

The family unit is the foundation of Saudi society, and due to good family structures and support, terminally ill patients seem to cope better at home here, than in other parts of the world (Younge et al., 1997). Almuzaini et al., (1998) found that although informal carers in KSA who were outside of the region of KFSH\&RC expressed dissatisfaction with cancer care, 97\% responded 'no' when asked if the burden of caring for the patient was too much for them. This is despite that fact the caring role had an impact of employment for $80 \%$ of informal carers. However, the negative answer may be due to social expectations about looking after the family. Family bonds are not only between parents and children but include the wider extended family to differing degrees (Almuzaini et al. 1998). It is notable in Saudi society that there is a determination on behalf of family members to look after their relatives when ill, especially the elderly (Almuzaini et al. 1998). This is a direct impact of Islamic religious belief, where the Quran states "Your Lord has decreed that you worship none but Him and that you be kind to parents. Whether one or both of them attain old age in the life, say not to them a word of contempt, nor repel them, but address them in terms of honour. And, out of kindness, lower to them the wing of humility, and say: My Lord, bestow on them thy Mercy, even as they cherished me in childhood" (Quran 17:23-24, cited in Almuzaini et al., 1998).

In summary, Hospice care has been central to the development of palliative care in other countries. However, it is not yet available to KSA, and this may be in part due to the perception by patients of a hospice as similar to a care home or nursing 
home, which many Saudis would reject on religious and social grounds, as it is important both religiously and culturally to care for family, preferably within one's home ( Almuzaini et al. 1998). This may mean that the hospice system is not appropriate for KSA. However, given the numbers of palliative care patient dying in acute hospitals, which may not be the most appropriate setting, education about the purposes of hospices may increase acceptance of this idea, particularly where the symptoms are too difficult to be managed in at home.

Muslims are not allowed to hasten death through medication and may express fear of pain relieving drugs such as opiates if they suspect they may this impact. An open and factual discussion should take place with the family in this instance (Mohammad Zafir Al-Shahri, 2002).

The relationship between the doctor and the patient is different in KSA to in Western countries, where the individual rights of the patient are paramount. In KSA the patient is viewed as one element of the family unit, and the family have a role to play in how much or little the patient knows about their condition (Younge et al., 1997).

This dynamic is demonstrated within different relationships in a description of an ethical dilemma within an American hospital, concerning a family from KSA. Realising that their small baby was being kept alive only by a ventilator, the father asked for the ventilator to be withdrawn, but for the mother not to be told that this was the reason for death. It was postulated that within the context of KSA culture, the husband was attempting to shoulder the burden of a difficult decision, thereby protecting his wife (Sayeed et al., 2012). The concept of 'truth telling' in medicine 
comprises various concepts, including the right of patients to receive clear information about their condition, balanced against the imperative to do no harm, the right not to know, and the wishes of the family to protect the patient (Al-Amri, 2010). While in developed countries, the issue of informed consent has tipped the balance in favour of the right to know, the culture of KSA is one in which the family may try to protect the patient from knowledge of their prognosis. Firth (2001, cited in Gatrad \& Sheikh, 2002) observed that various non-Europeans, including Muslims, preferred non-disclosure to avoid loss of hope. However, a more recent study published by Al-Amri (2010) interviewed patients with an author constructed questionnaire after confirmation of diagnosis but before informing the patient. They found that $98 \%$ of Saudi cancer patients wanted to know about their diagnosis and prognosis. This preference did not appear to be associated with gender, the age or education level (Al-Amri, 2010).

\subsection{Respect}

In the Saudi culture, respect for elders is very important, and health professionals are recommended to continue this within a hospital setting, being soft spoken and humble towards elderly patients. Other things that can be interpreted as disrespectful towards all Saudi patients include laughing in front of the patient and entering the patient's room without permission. It is also preferable to use the right hand for administering medicine. Visiting the sick is highly recommended in Islam, and Saudi patients may often have many visitors at one time. It is not culturally acceptable for the patient to ask them to leave (Mohammad Zafir Al-Shahri, 2002). 


\subsection{Gender interactions}

The modesty of both males and females and limits between the genders are extremely important in KSA. The healthcare provider should be of the same gender, and where this is not possible, precautions should be taken such as having a person of the same gender present and only uncovering the amount of the body necessary (Al-Shahri and Al-Khenaizan, 2005).

Wards in KSA are either male or female and should remain so under all circumstances. Unnecessary touching between the genders is forbidden, and Saudi females may prefer to communicate with male health professionals via a male family member. This should not be viewed by health professionals as a sign of abuse, as it may be in the Western culture. Interpreters are often needed, and this interaction is likely to work better if the interpreter is the same gender as the patient (Mohammad Zafir Al-Shahri, 2002).

The avoidance of eye contact by patients with opposite gender healthcare providers should not be interpreted as a lack of respect, in fact, for Muslims, this is a sign of respect and modesty. A lack of affection between a Muslim patient and their spouse should not be taken as indicative of family dysfunction, as public displays of affection are unusual among Muslims (Al-Shahri and Al-Khenaizan, 2005).

\subsection{A good death}

A study was undertaken in KSA by Tayeb (2010), which sought to define elements of a "good death" as perceived by Muslim patients and healthcare providers of varying nationalities, and compare this to the 12 elements of a good death which have 
been identified as applying to Westerners (Age Concern 1999, cited in Tayeb 2010). Many elements were the same. However, several aspects which were relevant to Westerners were found not to be as important to Muslims, for example knowing when death was coming and what to expect. In addition, three novel elements were found to define a good death in a Muslim society. The first of these was faith, belief and preferences during dying, for example, dying in a holy place, and the presence of someone recited the Quran. The second regarded the patients' selfesteem and image to others, including continence and the general cleanliness. The third element was regarding the comfort of the relatives after death, with regards to economic and social concerns. Similarities with the Age Concern elements included control over pain and other symptoms. While Muslims perceive suffering as atonement for sins, and this helps the patient and family member to cope, it does not detract from the need for relief from pain and suffering.

\subsection{Cultural competence of nurses}

In addition to knowledge of palliative care, healthcare professionals in KSA, the majority of whom are from overseas, need an understanding of the culture and religion of KSA, in order to deliver effective palliative care. Such cultural competence is seen as a quality indicator within nursing, and studies into cultural competence in KSA have focused on nursing. Studies have shown that low cultural competence can result in dissatisfaction with care and poor outcomes (AlYateem and Al-Yateem, 2014b). Little is known about the knowledge of overseas nurses in KSA in regards to the dominant culture and religion. In a qualitative study to explore nurses experiences of caring for patients in KSA, AlYateem \& Al-Yateem (2014) 
collected written narratives based on open-ended questions from 27 nurses recruited from two hospitals. Within this study, most of the participants demonstrated insufficient knowledge and experience of Islam, which prevented them from delivering culturally competent care. There was also a tendency for nurses not to recognise religion as a part of the healing process, and for them to see it as something which hinders their ability to give care. This was not the case with all participants, and some of the nurses recognised the patients' right to practise their own religion and desired balance between this and the priorities of the nursing care. Most participants acknowledged a need for knowledge about the culture and religion of their patients and felt that it was lacking. This had caused some nurses to look for knowledge from different places, sometimes resulting in misinformation. The participants reported communication as one of the most distressing aspects and felt hindered in delivering good nursing care due to not speaking the patients' language (AlYateem and Al-Yateem, 2014b).

Sidumo et al. (2010) found a similar lack of cultural awareness in foreign nurses working on obstetrics units. Using a quantitative descriptive research design, it was found that nurses lacked, among other things, awareness of diseases specific to the population, and common cultural practices. An example of how this can create misunderstandings is on the issue of breastfeeding. Many nurses described Saudi mothers as 'unwilling' in regards to breastfeeding. They did not take into account that modesty issues may have prevented the women from breastfeeding in front of other people. While this study was within a different medical area, it gives a good 
example of how cross-cultural misunderstandings can lead to misperceptions between nurses and patients.

A paper by van Rooyen et al. (2010) presents the perspective of South African nurses working in KSA, based on diaries and qualitative interviews. This offers a different perspective, identifying issues that may need to be addressed. Certain religious practices caused feelings of discrimination and unfairness, for example, non-Muslim nurses were expected to work longer hours than Muslim nurses during Ramadan, but were forbidden from eating and drinking in front of non-Muslims, causing discomfort and even dehydration. They were also expected to provide patient cover for Muslim nurses while they prayed. In terms of nursing practice, they felt disadvantaged, not only by difficulties in verbal communication but also by being unable to use certain aspects of non-verbal communication, such as eye contact and touching, due to cultural limitations.

Aldosh (2015) aimed to address the training need in nurses, by designing a practicebased culturally sensitive model of health education for hospital nurses. Based on the findings of interviews with nurses, which identified problems lack of time and staff shortages, language inconsistencies between nurses and those training them, and communication, language and psychological states of the nurses themselves, Aldosh (2015) recommends a two-level approach to this problem, a within hospital intervention, including education, removal of barriers, and motivational interventions, and a community level intervention, including updating policies, cooperating with community organisations and the use of media. Due to the level of cooperation that this model involves, it would need to be tried, tested and 
modified accordingly. This Aldosh PhD research has a strong methodology, using multiple methods. However, given the failure in the past of KSA to take on board suggested policies and changes in palliative care, it may be difficult to put the suggested model into practice.

\subsection{Policy changes in the KSA}

Historically, policy changes in the KSA happen through a gradual process of bureaucratic acceptance (Bjerke and Al-Meer, 1993). Change is difficult in Saudi Arabia due to the deeply ingrained power distance between the ruling class and the common citizenry. Decisions are made based on ideals that are held onto by the former, and are reflected in various policies and programs of government; these remain in place unless the ideals of those in authority change, or they are succeeded by the next in line (Bjerke and Al-Meer, 1993). However, more recent writings on political dynamics in the KSA hint towards some changes. As explained by Alhirz and Sajeev, (2015), policy changes in the country have come to be shaped more significantly by continuous evaluation of outcomes from the implementation of existing policy. Within the past decade, various ministries within the KSA government have engaged in a series of cyclical developmental programs, where they introduce changes to existing policies and then gather data on the impacts of these changes within an allotted period of temporary implementation. This is supported by evidence gathered in the work of Dulayami and Robinson, (2015). While Dulayami and Robinson, (2015) focused on the private sector, both their work and the work of Alhirz and Sajeev, (2015) substantiate increasing drive towards scientific bureaucracy in the KSA, where there is a greater premium placed 
on gathering empirical evidence to support the actual benefits brought about by changes in policy. This has considerably improved the dynamics of policy changes in the KSA.

\subsection{Comparing KSA to the UK}

To understand the palliative care situation in KSA, it is useful to compare provision to that of the UK. The concept of palliative care is still relatively new in KSA as well as the rest of the Middle East. This is in contrast to the UK, which can be seen as the soil from which palliative care grew, given the role of Dame Cicely Saunders and the hospice movement in establishing current practice.

Three major service sectors make a contribution to palliative care provision in the UK, the National Health Service (NHS), social care and the charitable sector. Policy has been informed by expert opinion, a range of expert views being aggregated under a single organisation, the National Council for Hospice and Specialist Palliative Care Services (NCHSPCS).

In 1990, the World Health Organisation (WHO) set its first palliative care standards, which were revised in 2002. The National Standards were agreed in the UK in the 1990's. The NHS offers palliative care that is designed at "attempting to relieve pain and other distressing symptoms while providing psychological, social and spiritual support." (no date: online). This is designed for people who have an illness where there is no cure and is may be in many forms. Palliative care is now a recognised sub-speciality of Medicine and has its own training programme and exit exam (Fallon and Foley, 2012). 
Palliative care in the UK has been primarily associated with cancer patients.

However, it is now recognised that patients needing palliative care for other reasons although it appears they can have difficulty accessing the services. Policy is changing to reflect this (Mathew et al., 2003). Fallon \& Foley (2012) cite the following points as considerations in transforming palliative care to be accessible for people suffering from non-cancer diseases:

- Close and meaningful collaboration with specialists from other disease areas,

- Research to increase the evidence base for appropriate palliative care in other diseases,

- The focus should be moved away from just end of life care, to involvement at earlier stages in disease as this has proved to be more effective in the case of cancer and

- The significance of the difference in palliative needs between cancer and other life-limiting diseases should be acknowledged in hospitals, residential homes, as well as in hospice.

The 2015 Quality of Death Index (The Economist, 2015) ranked the UK the overall top provider of palliative care in the world, "thanks to comprehensive national policies, the extensive integration of palliative care into its National Health Service and a strong hospice movement" (p6). In contrast, KSA was ranked $60^{\text {th }}$, and the Philippines, where many nurses in KSA originate, was ranked $78^{\text {th }}$. At this time KSA was noted to have a limited availability of opioids. 
This is not to say that there is no further room for improvement in the UK, and the report notes that "even the top ranked nations struggle to provide adequate palliative care services for every citizen" (The Economist 2015, p6). A report from the Royal College of Physicians (RCP) reports problems including communication with patients about the fact that they were dying, lack of palliative care at weekends, and insufficient fluids being given (Boseley, 2014). It was reported that $75 \%$ of family members feel involved in the care and treatment of the patient, which is positive but leaves room for improvement, and the report also reported a lack of training for palliative care staff (Boseley 2014).

\subsection{KSA issues in a wider context}

Attitudes and approaches towards death and dying care vary widely between different religions and cultures, influenced by patients, their families and caregivers. Previous sections of this PhD have explored the impact of the culture of KSA on the palliative care situation. There has also been research conducted into culture and religion in palliative care, focusing on ethnic minority communities in the UK and USA. While this may not, at first glance, seem relevant to the discussion of palliative care in KSA, there are parallels that make the research interesting to review. The healthcare, and particularly the palliative system in the UK and USA, have been developed according to Western principles, heavily influenced by Christianity. Ethnic minority culture and religion may be at odds with some of these principles. In KSA, the patient population is Muslim, but the majority of healthcare professionals are not, and are not native to KSA. Therefore, while the cultural and religious situations are not identical some of the issues will be similar. In the section 
covering palliative care literature in KSA, the cultural and religious issues which impacted upon the delivery and the experience of palliative care within KSA were discussed. Cultural issues included language and communication, family, truthtelling and perception of pain management. Religious issues included education of healthcare professionals in Islam, and respect for their patient's faith, and the death rituals used by Muslims. The following sections will expand upon the discussion of culture to include international literature. It will look at the place of spirituality and religion within palliative care, which may offer insights as to the role of Islam in palliative care in KSA.

\subsubsection{Culture}

Culture has been defined as, "a relatively specialised lifestyle of a group of people, consisting of their values, beliefs, artefacts and ways of life, and communication that is passed on from one generation to the next."(DeVito, 1992:58). The discussion around a definition of culture is complex, but this straightforward definition meets our current needs. According to his professional observations, Nyatanga (2002) suggests that cultural perspectives influence the way that patients view health, illness and treatment and may underlie differences in seeking treatment, the taking of medication, and responsibility towards health. Palliative care will be interpreted differently throughout the world, according to factors such as economic considerations, differing healthcare systems and availability of treatments (Nyatanga, 2002). 
It has been suggested that a model of palliative care should ensure the care offered is culture specific and patient centred, and arguably this will be more likely to be achieved with care providers from the same cultural background as the patients (Nyatanga, 2002). This is not always possible, but it is at least important that healthcare professionals have a level of cultural and religious awareness in order to provide effective palliative care (Mir, 2011). Language can be a particularly difficult barrier to overcome in providing palliative care to ethnic minorities, and both professional and informal interpreters can be used (Nyatanga, 2002). These points reflect the observations of authors in regards to KSA (Mohammad Zafir Al-Shahri, 2002; AlYateem and Al-Yateem, 2014b).

Styles of communication differ between cultures, and an area of communication which affects healthcare is that of truth disclosure to patients with serious illnesses. The centrality of the concept of informed consent in the West means that truth telling in regards to health is the norm. Many Middle Eastern countries have a tradition of withholding information from patients to maintain hope, yet legislation in these countries is now changing to reflect the growing importance of informed consent, and therefore such countries are in transition (Bou Khalil, 2013). The literature suggested that in KSA, many patients are still unaware of their diagnosis, despite a study by Al-Amri (2010) suggesting that $98 \%$ of Saudi patients wished to know their diagnosis.

Bou Khalil (2013) undertook a review of the literature concerning truth telling in relation to cancer in Middle Eastern countries. Fifty-five papers were reviewed, and this included papers that were identified as having weaknesses in methodology, 
however, due to the lack of literature in this area and the importance of including whatever information was available these have been included. The overall findings suggested that opinion still leant towards concealment rather than disclosure, though there did seem to be a recent shift towards a greater appreciation of disclosure. The author acknowledges that the legislation used in the West to ensure truth telling may not be appropriate to Middle Eastern culture, but feels it is essential that Middle Eastern countries develop their legislation in regards to this. In KSA this issue appears to be linked to the family, who play a role in deciding how much the patient knows about their illness (Younge et al., 1997). In Muslim countries and cultures, the extended family structure is the norm, and family dynamics differ considerably to those of the West, often being hierarchical and patriarchal. Males and females often have different and complementary responsibilities within the family (Gatrad and Sheikh, 2002), as has been discussed in detail previously about KSA. Although it is the right of every mentally competent adult to make their own decisions regarding healthcare, in reality, in KSA family members may contribute significantly to the decision-making process (Al-Shahri and Al-Khenaizan, 2005). Research has shown that Muslim patients would prefer access to palliative care in locations other than the hospital (Tayeb, 2010), Muslim families prefer to care for relatives at home, and Muslim patients prefer to die at home (Aljawi and Harford, 2012). Palliative care in an institution may be seen as 'giving up' or not fulfilling one's duty to care for the family (Schultz et al., 2012). Care of the dying is seen as an essential part of Muslim family life, with numerous 
sayings of the Prophet Muhammad (Peace be Upon Him (PBUH)) attesting to the virtue of caring for the sick and dying.

The impact of a lack of cultural understanding is evident in research on the use of palliative care by ethnic minorities in Western countries. Ethnic minorities have been found to be under-using palliative care services in proportion to their numbers (Nyatanga, 2002). Minority groups are at risk of not receiving good palliative care, including the under-treatment of pain. Nyatanga (2002) argues that education is key to helping health professionals to understand the needs of minority groups. This would also apply to non-native healthcare professionals in KSA, and similar observations have been made specifically about that situation. Regarding public awareness, engagement with the media is an important element of palliative care education, as the public get most of their knowledge of science and medicine from the media. However, it can be difficult for health organisations to engage with the media in the most constructive way (Wee and Jamison, 2014). The WHO designed a Public Health Strategy, for countries to use to raise awareness of palliative care, which includes education of the public. They suggest engaging the media to heighten public awareness about the aims and objectives of palliative care (Stjernswärd et al., 2007). No literature was available on the relationship between palliative care and the media in KSA.

\subsubsection{Spirituality}

Spirituality has been acknowledged as a component of palliative care from its inception. It is seen as a factor in 'total body pain', a term coined by Cicely Saunders (Mir, 2011), which can occur at the end of life when patients have not attained 
closure on issues in their life, ranging from financial issues to a relationship with a loved one. This model eventually came to be described as the biopsychosocial spiritual model (Puchalski, 2012). This model emphasises the totality of the patient's experience, spirituality being an equal domain with physical and other domains in the experience of dying for patients and families (Puchalski, 2012). It is suggested that spiritual pain can exacerbate physical pain, and therefore the two cannot be entirely separated (Kellehear, 2000). Spiritual care is still recognised as a component of palliative care within Western models of care (Al-Shahri \& AlKhenaizan, 2005), and in keeping with this, is recommended as one element of palliative care in both Department of Health (2008) and National Institute for Clinical Excellence (2004) care policy documents in the UK.

Interest in religion and spirituality in relation to physical illness has increased in recent years, according to an article in the Geriatric Times (Cohen and Koenig, 2002), but these issues have not been thoroughly addressed in research. However, some studies have provided evidence for an association between spirituality/religion and health outcomes, including quality of life and coping, and surveys have suggested that patients wish to have spirituality integrated into their care, demonstrating the importance of spirituality at this stage of life (Puchalski, 2012). A qualitative study conducted in Germany by Asgeirsdottir et al. (2013), aimed to examine the experience of spirituality and its influence on the lives and well-being of palliative care patients using qualitative interviews. The findings were that the meaning of God or other spiritual practices was a key factor in giving hope and strength and activating inner resources. 
Amoah (2011) presents an argument for the importance of spirituality in palliative care from reviewing the literature and his experience as a chaplain at an end of life hospice. While spiritual beliefs are generally believed to benefit people at the end of life Amoah (2011) notes that in some people such belief can be ineffective or problematic, such as the belief that an illness is some form of divine retribution. Spiritual needs are complex and may overlap with social and psychological needs, and may or may not be expressed within the context of religion. Kellehear (2000), also basing his views on a combination of literature and personal experience, has proposed a model of spirituality in palliative care based on three dimensions: situational, moral and biographical, and religious. Situational aspects include hope, meaning and social presence. Moral and biographical aspects include prayer, forgiveness and closure. Finally, religious aspects include divine forgiveness, religious literature and religious rites. (Amoah, 2011), has suggested that health professionals be aware and supportive of spiritual needs during dying, as it is more a spiritual event than a medical one.

Despite the obvious importance of incorporating spirituality into palliative care, there are many barriers to this. Practitioners may have ethical concerns about involving themselves in such a personal area of a patient's life and have concerns about how exactly it should be done (Puchalski, 2012). There is some debate about who is responsible for the spiritual aspect of palliative care. This role may traditionally have fallen upon nurses, but social workers and psychologists can also see this as part of their role (Kellehear 2000). Conflicting demands on health professionals also make it difficult for any one group to take sole responsibility. 
Spiritual care as a part of palliative care needs to be guided by the same general principles as palliative care, including good communication, assessment and care planning, symptom management, maintaining comfort and well-being, and advance care planning (Amoah, 2011). For example, good communication is key to assessing spiritual distress and planning for death, whereas lack of adequate communication may heighten distress and undermine the patient's experiences. Elements of appropriate communication include active listening validating emotions, and empathising (Amoah, 2011). Schultz, Baddarni, \& Bar-Sela, (2012) recommend asking patients directly about their spiritual and religious beliefs, to better understand the patient as a whole person. Puchalski, (2012) recommend "standardised education programmes for all clinical disciplines in spirituality and health." (p63).

Spiritual beliefs can find their expression in organised religion, or in some other form of belief. They can be used to help patients to find a way of coping with suffering and finding peace and wellbeing in the midst of dying. Kellehear (2000) notes that modern palliative care discourse has distanced itself from the discussion of God and religion, preferring the more neutral territory of spirituality. While spirituality includes religion, religion is not fully defined by spirituality. Spirituality divorced from God seeks peace in this life, but not necessarily the next. However, a commonality, according to (Kellehear, 2000) is the phenomenon of transcendence, that is, to find meaning beyond one's current suffering which allows one to make sense of the situation. In the context of KSA, a Muslim country, it can be assumed the majority of patients will frame their spiritual beliefs within the religion of Islam. 


\subsubsection{Islam}

Islam is a complete way of life, and permeates every aspect of the Muslims life: "The most pervasive influence of palliative care provided within an Islamic community is Islam."(Almuzaini, Salek, \& Nicholls, 1998, p371).

This can certainly be said to be true of KSA, where Islam has been central to the inception and development of the country.

A universal aspect of religion is that suffering should be minimised. As palliative care is a holistic approach to relieving the suffering of the patient, religion may play an important role in determining the goals of care, and again, this is even more likely to be true in a conservative Muslim country such as KSA (Mir, 2011). Garrido, Idler, Leventhal, \& Carr (2012) undertook a study to evaluate the extent to which religious affiliation and importance affected advance care planning, in the context of beliefs about control over length of life and values about end of life. It was expected that people who perceive themselves as having control over a situation were more likely to engage in health-related actions. They found that those placing great importance on religious values were less likely to engage in advance care planning. This was because of a belief about God controlling the length of life, and about using all available treatment options as a way of valuing life. Similarly, in KSA, there is no evidence within the literature that advanced care planning takes place. The study also found various factors that were highly valued by religious people at the end of life, including being mentally aware, knowing what to expect about ones' condition, freedom from pain, freedom from shortness of breath, and not being connected to machines. This study included patients from several religions, but 
mainly Christian and Catholic denominations. Islam shares some features with other monotheistic religions but also has distinct characteristics which mean that Islam has a unique impact on the health beliefs of Muslims, and their experience of death.

A paper by Choong (2015), a reader in medical ethics at Lancaster University, provides a useful perspective on whether modern pain management as part of palliative care is consistent with Islamic values. The paper addresses two central questions: is pain relief permissible when, in Islamic discourse, pain and suffering lead to the expiation of sins, and; is it permissible to use pain killers which can bring about addiction, impaired consciousness and shortening of life, all of which are forbidden in Islam? They conclude that while it is unanimously agreed by Muslim scholars that it is permissible for Muslims to seek pain relief, the intention of administering such drugs must be with the primary intention of pain relief, and not exceed what is needed for this purpose (Choong, 2015).

The four basic concepts of medical ethics are congruent with an Islamic perspective on healthcare: non-malfeasance; justice; autonomy and beneficence (Al-Shahri and Al-Khenaizan, 2005). From an Islamic viewpoint, death is accepted as an expression of Gods' will (Gray et al., 1995), and the timing of death is predetermined by God and therefore not under the control of human beings:

"It is not possible for a soul to die except with the permission of God at a term set down on record." (Quran 3:139).

This means that the right to die voluntarily is not validated in Islam. As Muslims believe in the day of judgement, and the afterlife, death is accepted as part of the 
divine plan, and rather than an end point, is a point of transition (Choong, 2015). Dying is a time for reflection and repentance, and seeking closeness to God through prayers and recitation of the Quran. It is also a time for seeking forgiveness from God, and also people, for any past transgressions (Choong, 2015).

Sickness is a reminder to the believer, to assist them in leaving sins and contemplating the eternal afterlife. Rather than seeing themselves as abandoned by God in their sickness, Muslims know that God tests the believers by the trials of this world (Schultz et al., 2012), and that patience in suffering is rewarded in the afterlife (Choong, 2015). Similar observations were made by authors in regards to KSA Muslims (e.g. Babgi 2010; Almuzaini et al. 1998)

Islam views life as a sacred trust, making suicide and euthanasia forbidden (Gatrad and Sheikh, 2002). However, this does not prevent a believing Muslim from seeking a cure for the disease, as the Prophet Muhammad (PBUH) also said: "O you servants of Allah take medicine as Allah has not created a disease without creating a cure except for one (old age)."(Al-Shahri and Al-Khenaizan, 2005). While drugs that affect sensorium are strictly forbidden in Islam, opioids are considered acceptable due to necessity (Al-Shahri \& Al-Khenaizan, 2005). The relief of pain for the Muslim patient may enable them to engage in spiritual acts that would be impossible if in severe pain (Choong, 2015). However, as will be further discussed, patients and families may have reservations about using such drugs and need clear explanations as to their purpose (Choong, 2015). 
Advanced medical directives allow the patient to plan for important decisions in end of life care, at a time when they are still well enough to make those decisions. One such example may be to choose a 'do not resuscitate' policy, in the event that it would only extend life when the quality of life is poor (Al-Jahdali et al., 2012). AlJahdali et al. (2012) considered the congruence of such directives with the Islamic faith, and issues around Muslims using such directives. An important aspect of Islam is 'Qadr' (pre-destination), and through this, Muslims view sickness and death as an expression of God's will, to be borne with patience. As previously discussed, Muslims are also obliged to seek remedy for illness, and according to Al-Jahdali et al. (2012), there are three levels of this obligation. To seek a remedy where there is proof of benefit for oneself or others is obligatory, where treatments may or may not help prolong life or improve one's condition, seeking treatment is optional, and treatment should not be sought where it would be futile. The authors believe that despite this, Muslims may be reluctant to discuss issues of life and death, due to the belief that it is in God's hands, and for fear of going near the issues of suicide and euthanasia. According to (Tayeb, 2010) most Muslims are unaware of the concept of advanced directives. However, Al-Jahdali et al. (2012) describe how the Prophet Muhammad's own actions in the time near his death, indicate the permissibility of advanced directives. He was known to have refused medicines during his terminal illness, and to have corrected those who (out of love) tried to force feed him medicine. Muslims use the Prophet's life as a model for their own, so education about this issue could help promote the use of advanced directives among Muslims. In a country such as KSA, there would also need to be an increase 
in open honesty about the patient's condition, in order for advanced directives to become a reality.

(Mir, 2011 p168) states that, "Cultural and religious awareness on the part of the healthcare team is needed to provide patients with effective end of life palliative care". Even so, patients are individuals and stereotypes should be avoided, and therefore care of each patient should be individualised, to produce a plan which fits within the cultural and ethical framework of the patient. From the Muslim's perspective, Islam has several values in common with the palliative care movement, including the belief in the inherent value of life, and caring for the sick. The family centred approach found in many Muslim communities is also in congruence with the palliative care remit and may benefit the establishment of palliative care (Choong, 2015).

\subsection{Summary}

This chapter began by outlining the definitions and history of the palliative care movement, before providing a comprehensive review of literature relating to palliative care in KSA, which emphasised the inadequacy of current provision, and highlighted particular salient issues. This was then compared to current provisions in the UK. The chapter continued by building on these issues, then presenting a sample of international literature concerning palliative care in relation to culture, spirituality and Islam.

Challenges to palliative care in KSA exist on various levels. They exist at the professional level, where practitioners may neither be sufficiently equipped nor 
sufficiently oriented to deal with palliative care concerns. Saudi practitioners may have misconceptions that lead them to recommend or carry out activities that may be detrimental to the palliative care of their patient. Issues also exist at institutional levels, where palliative care systems that are in place in Saudi hospitals are insufficient for addressing the needs of its patients. There are also issues found at the cultural and legal levels, where pain and individual choice are not considered as an important enough concern in Islamic law especially when weighed against matters about prolonging and preserving life and preparing the individual for the afterlife. As such, even if there were competent practitioners of palliative care in KSA healthcare institutions, their hands are tied from doing or prescribing what should be the best course of action for a patient's comfort, dignity and well-being, by the cultural-based wishes of the patients' family even against that patient's will or to keep the patient from becoming addicted to pain medication.

The current study seeks to uncover issues that may prevent such practitioners from seeing the value of palliative care nursing and to provide suggestions which may be used by administrators as a basis for developing programmes that will better orient practitioners to the relevance of palliative care in today's healthcare setting. 


\section{Methodology}

The methodology guides the methods used to collect and analyse the findings. In this chapter of the thesis, the methodology for the research to be undertaken will be discussed in detail, and the methods used to undertake the research will be described. Initially, the research aims are reiterated and the researcher's position, in relation to the research, is described. Then the research paradigm, theoretical perspectives and methodology are discussed, which leads to a detailed description of the methods used to undertake this study. Finally, the chapter addresses the issue of evaluating quality in qualitative research.

\subsection{Research Aims}

The aims of this study are threefold:

- To determine the perceptions of KSA hospital healthcare professionals regarding palliative care,

- To identify issues regarding the development of palliative care in the KSA hospital setting, and

- To develop recommendations for strengthening the value of palliative care among healthcare professionals in KSA.

\subsection{The researchers' position}

Denzin \& Lincoln (2011), suggest that the researcher has a significant impact on the design of the research, due to their position in relation to the subject of research. Etherington (2004) describes research reflexivity as "the capacity of the researcher to acknowledge how their own experiences and contexts (which might be fluid and 
changing) inform the process and outcomes of inquiry." (p.31). Rather than seeking to eliminate the effects of the researcher, one can use reflexivity to understand and report these effects (Holliday, 2007). By being aware of oneself in the research, one can limit the effects of oneself on the data (Lietz et al., 2006). Therefore, in this section, the researcher's own position will be outlined.

After achieving a degree (master of advanced practice - palliative care strand) in Australia, the researcher went back to KSA to work. After seven months a position was secured, which gave the opportunity to work within palliative care in the researcher's home country and enabled the application of the principles learnt from the study abroad. Unfortunately, this did not enable fulfilment of the patient's needs or promote the development of the role. The workload was expanded to Wound Care, and IV patients, and, as the work was home care based, and appointments were geographically spread, there was little time to spend with patients. This goes against the nature of palliative care, which requires more time with patients. This situation would be difficult anywhere, but in an area, with no street names or satellite technology, it was impossible to fulfil the duties assigned. In regards to palliative care clearly huge improvements were needed, and it was obvious health professionals in KSA were not aware of palliative care. They did not engage in its practice, and as a result, they did not recognize its value and its importance.

The wider organizational environment in healthcare in KSA can also make working there very difficult, and affect the quality of healthcare available. Al-otaibi (2014) in a study of the organizational culture within healthcare in KSA states that "A primary 
cause of problems associated with healthcare provision in Saudi Arabia today is that it is heavily influenced by the prevailing organizational culture within the country" (p1898). The study found that hospitals were dominated by a culture of hierarchy.

Things were made very difficult in this role, and, as a Saudi nurse in a health system with a hierarchy order based on nationality, there was no footing to change the local situation, let alone that of the flawed healthcare system. For this reason, the researcher decided it would be more beneficial to complete further studies and contribute to research.

After this experience of working as a nurse within palliative care in KSA, it seemed clear that a deeper understanding of the phenomenon (the underdevelopment of palliative care in KSA) was needed. After investigating the literature regarding palliative care in KSA, nothing in the Arabic languor, and few in English were found, and of those found many were reviews rather than pure research. This reinforced the impression that the issues of establishing the infrastructure to palliative care were not being addressed. The literature review suggests KSA has not yet developed a formal model of palliative care which is applied throughout the whole country.

Based on these, the researcher has opted to assume the position of an involved stakeholder operating within the environment of interest. As a nurse who has worked in the KSA, the researcher knows the study setting personally and works with the people that the study intended to gather data from. Thus, the researcher acted as a fellow healthcare provider to the study's participants, understanding 
their perspectives and engaging them in discourse rather than simply gathering data from the point of view of an outsider looking in. This position allowed the researcher to explore inputs of participants more intensively, being able to relate to participants and offer own experiences to deepen discourse. At the same time, however, this position exposes the researcher to personal bias in gathering data from participants. Since the researcher has a personal stake in the study setting as a fellow healthcare provider, there may be situations where the researcher's personal perspectives are introduced to the data. However, the researcher has taken care to identify these situations as they arise in order to be able to openly account for any personal perspectives that the researcher may be injecting and avoid such biases from being left undetected and unaddressed in the analysis.

\subsection{Paradigm, theoretical perspectives and methodology}

\subsubsection{Paradigm: Subjectivist leading to qualitative research}

Having established the position of the researcher in engaging with the research environment, this next section works towards establishing the theoretical foundations on which the researcher undertook the study. Ontologically, this study is founded on the subjectivist paradigm that knowledge cannot exist outside of the people that possess it, and that people generate their own knowledge based on their own, individual perspectives, which is mediated by the world that they find themselves in, and their background and experiences (Braun and Clarke, 2006). This foundation dictates that every person, each doctor and nurse working in hospitals in the Kingdom of KSA, has important inputs that they can share in this study about palliative care. As such, this study must proceed to extract such information directly 
from respondents by capturing their perspectives in the study. It is important for the study to determine what doctors and nurses think are the salient issues in relation to palliative care in the country, as well as what they think about those salient issues. Only those doctors and nurses can provide valid information about what they think the issues are and how important each of these issues is to their profession. Furthermore, it is expected that the contributions of these doctors and nurses are not the same and may be quite diverse. In fact, there may be some contributions of some of these people that are significantly in contrast of those by other people in the same population, yet each of these contributions is equally important.

The research questions of this study focus on highlighting the experiences and opinions of a particular group of people, that is, health professionals in KSA. Both quantitative and qualitative methods could be relevant to this area of research. While some see them as opposing positions, a more balanced view is that they can be complementary to each other, each bringing a different insight to a problem. For this study, a qualitative design was preferred over a quantitative design. This is consistent with the subjectivist ontology that was adopted in this study.

In qualitative research, the main objective of the investigation is to explore the perspectives of representatives of the intended population intensively, to draw an accurate description of their view of the phenomenon of interest, which in this case is palliative care in KSA hospitals. The qualitative design was selected over the quantitative research design primarily because the former suits the aims of the research better. This is because the aims of this study are finding out what the 
positions of different healthcare professionals in KSA were about palliative care, and then determining what can be done in order to strengthen palliative care in the country. These aims give rise to research questions that cannot be answered as effectively using a quantitative design. For example, in describing the perspectives of different healthcare professionals in the country about palliative care, using a quantitative design implies that an instrument can be designed that can encompass all of the possible perspectives that such professionals may have, making it a simple matter of gathering data on the extent to which different professionals have each of these different perspectives through a survey. However, based on the review of the literature, the perspectives of physicians about palliative care can be quite diverse, and there are no limits that can be set in a specific context as to what these perspectives may or may not contain. In addition, as yet there is no agreed understanding of what they may be in KSA. As such, the use of a quantitative design may gravely limit the outcomes of the study to the extent that such outcomes would not validly represent the actual perspectives of the population of interest.

A qualitative design, on the other hand, does not set a limit to what can and what cannot be found in gathering data from respondents. Rather, it allows the research to examine respondents' perspectives bounded only be respondents' consent, and their willingness to articulate what they really feel.

Another reason for selecting a qualitative design for this study is the limited amount of quality research into palliative care in KSA that was found. From the literature we know that despite the efforts of a small number of key people to develop palliative care provision, progress has been slow, provision is patchy, and 
many people in KSA will not have access to effective palliative care when needed. There is a need to understand the reasons behind this, and in order to do this, the voices of ordinary healthcare professionals in relation to palliative care need to be heard. Therefore, this study required the collection of deep, rich data, as found in qualitative research. As such, the qualitative design was considered to be more appropriate for this study. At the same time, qualitative research can be seen as a necessary precursor to quantitative research, in order to identify relevant areas for research, whereas others see qualitative research as having a value in its own right (Glaser and Strauss, 1967). The research design that was selected for this study is the qualitative research design, reflecting the subjectivist paradigm. As the human being needs to be understood in relation to the time and place and other contextual factors, the objectivity of a quantitative method is no longer desirable (Denzin \& Lincoln, 2011). Therefore, the researcher moves away from being merely a tool within a process designed to uncover a perceived objective reality, to being a researcher keen to uncover subjectivity which is valued and used within the research process (Charmaz, 2006). Thus, by engaging in qualitative research, this study hopes to open avenues for further quantitative studies; establishing vital groundwork upon which further academic explorations may be made.

\subsubsection{Theoretical perspectives: symbolic interactionism and grounded theory}

While a qualitative design was selected for this study, it remained important to identify specific quantitative perspectives that the study would adopt. In line with this, the theoretical perspectives of Symbolic Interactionism and Grounded Theory were selected. Both of these perspectives contain ideas that were found to be most 
relevant to the context of the research. Within Symbolic Interactionism, reality is considered to be borne out of social interaction (Charon, 2004). People do not respond directly to reality, but through their social understanding of this reality (Charon, 2004). Therefore, to be able to capture aspects of this reality, a researcher must derive data from those who experience this reality through interaction with them (Charon, 2004). In line with this, people act or refuse to act, based on the meaning that the place upon such actions and inactions, which are in turn formulated from their interactions with others. This cyclical process is present in any aspect of society, and so it must be present in the context of doctors and nurses involved in palliative care in KSA hospitals. Drawing from the work of Burbank \& Martins (2010)on Symbolic Interactionism as applied to the nursing profession, the following five premises were adopted.

First, the respondents of this study, doctors, nurses, and hospital administrators, must be understood as social beings (Burbank \& Martins, 2010). As such, their actions are dictated not just by a set of static rules, but by their dynamic with other people around them. In order to be able to understand their perspective about palliative care, their experience with other people in relation to this phenomenon must be taken into consideration.

Second, the respondents of this study must be assumed to be rational, thinking beings (Burbank \& Martins, 2010). That is, their actions and decisions are not random, nor are they simple reactions from their interactions with others, but are just as much the products of their interactions with themselves. In addition to this, the actions and perspectives of a doctor or nurse with regard to palliative care 
cannot simply be considered as a result of the rules set by the establishment where they operate, such as the hospital. Therefore, simply considering these rules does not provide an accurate idea of what the perspectives of doctors and nurses with regard to such rules are. Enquiries must be made about what these professionals think about palliative care, the depth of their understanding of the concept and the extent to which they have actually experienced the concept in their working lives. Third, the respondents do not sense their environment directly; rather, they sense it based on the situation that they are presently in (Burbank \& Martins, 2010). As such, in drawing out data from doctors and nurses, it is important to understand the position that they are in, and what this position does to their perspective about palliative care.

Fourth, the reasons behind doctors' and nurses' inputs about palliative care are based on their interactions with the present, rather than their retrospection with the past (Burbank \& Martins, 2010).

Finally, it is assumed in this study from the philosophical paradigm adopted, that respondents are active in relation to their environment (Burbank \& Martins, 2010). The doctors and nurses are actively involved in their professions, and therefore provide an insider view of the palliative care phenomenon in this context, in the particular cultural and religious context of KSA.

On the other hand, Grounded Theory seeks to construct a theory about issues of importance in peoples' lives (Glaser \& Strauss, 1967), by letting those issues emerge through the collection of data, rather than starting with preconceived ideas (Mills et al., 2006). Rather than starting with formulating a hypothesis and then collecting 
data to check the hypothesis' validity, the grounded study approach collected data first, and then from the analysis of this data, came up with a grounded theory which may be considered as the hypothesis of the study. As such, this enabled qualitative researchers from the grounded theory approach to approach a research problem without the need to establish any initial perspectives about the phenomenon of interest. Rather, they assume that they have no knowledge about the phenomenon, and then discover what there is to be discovered from the data that they gathered. While it is debatable whether this is practically possible, as researchers into an area are likely to have a prior interest in that area, and therefore observations and opinions, the emergent approach may help to address the accusation that qualitative research simply sees what it wants to see. Second, Glaser and Strauss (1967), in their description of the grounded theory approach, introduced a means to gather and analyze data in a fashion that is more objective and systematic than how data had been gathered and analyzed in the qualitative tradition. The focus of this method is the systematic generation and verification of themes, where a theme is a concept that is derived from the data gathered. Under the grounded theory approach, it is expected that gathering data from the population of interest, about the phenomenon of interest, will enable the researcher to draw out different themes, which collectively emerge from the inputs of the respondents. As the study gathers more data, more themes are expected to emerge, and in the relationship of these themes, theories that are relevant to the phenomenon of interest may be formulated (Glaser \& Strauss, 1967). 
Grounded Theory was intended by its original creators, Glaser and Strauss (1967), to be a complete methodology resulting in a 'theory' grounded in data. However, different forms of grounded theory have since been developed (e.g. constructivist grounded theory, (Charmaz, 2006), Corbin \& Strauss 2008), and many researchers now draw upon the principles and methods of Grounded Theory, without using the methodology in its complete form. Initially, the researcher intended to use a Grounded Theory methodology, but as the research design developed, it became clear that more flexibility in methods was required. However, this research has been influenced by certain elements of Grounded Theory. In grounded theory, the issues of importance are not preconceived and emerge through the data collection, and therefore is suitable where there is little prior research in the area. A coconstructionist perspective (Charmaz, 1990) views the findings as a co-construction between the researcher and the participant. This is highly relevant to the present study, as the researcher is himself a palliative care nurse, and will be interviewing other palliative care healthcare professionals.

In summary, then, the purpose of the investigation should be focused on developing theories as explanations for a phenomenon of interest, which are grounded on the inputs of relevant stakeholders in that phenomenon. In this study, the phenomenon of interest is the underdevelopment of palliative care in KSA, a phenomenon which is well established in the extant literature (Abu Zeinah et al., 2013; Alsirafy et al., 2008; Al-Shahri, 2009). The stakeholders identified to be involved in this study were different healthcare professionals in the country, as well as the patients. Among these, the study was limited to healthcare professionals, 
since they were most accessible for the study. It is highly unlikely that hospitals would allow a researcher to conduct data gathering on its patients due to restrictions that are inherent in hospitals in KSA. Specifically, the research is focused on the perspective of a specific set of actors, doctors and nurses, in a particular phenomenon, palliative care.

An essential part of this research is to explore the experiences of healthcare professionals regarding the palliative care patients. Thus, by being with the healthcare professionals, the researcher could get critical data about their experiences with palliative care. Since the research is aimed at giving suggestions for future changes in the field of palliative care by healthcare professionals, the examination of experiences play a vital role (Broback and Bertero, 2003).

\subsubsection{Methodology: pragmatic and thematic analysis}

The methodologies underpinning the methods of this research can be seen as pragmatic in approach. That is, rather than adhering to a particular theoretical standpoint, the researcher has gained an understanding of theoretical perspectives and applied those most appropriate for achieving the aims of the study. The analysis of the data has been informed predominantly by Thematic Analysis. Thematic analysis is a way of analyzing qualitative data which enables close engagement with the text in the first instance, and systematic stepping away from it so that the underlying meaning can start to emerge. It was developed as a method of qualitative research that provides a means of interpreting data (text) in a rigorous and conscientious manner (Fertuck, 2007). Through its theoretical freedom, thematic analysis provides a flexible and useful research tool, which can 
potentially provide a rich and detailed, yet complex account of data (Braun and Clarke, 2006). Compared to other methods of analysis, for example, Interpretative Phenomenological Analysis (Smith et al., 2009), thematic analysis can seem relatively descriptive (Wolcott, 1990). That is, it takes the statements of the interviewees at face value. This is one of the reasons for using thematic analysis for this research, as the aim is to allow the voices of health professionals of KSA to be heard. However, a level of abstraction is necessary and desirable, and this comes from comparing and contrasting the perspectives of the participants, for example forming possible explanations for different opinions on a matter.

\subsection{Ethical Considerations}

Ethical consent to undertake this study was obtained from Manchester Metropolitan University, Faculty Academic Ethics Committee (See Appendix 2). The issues covered by this process are relevant to research in the UK and do not necessarily reflect the complexity and differences in doing research in a country such as KSA. However, research ethics is a globally held body of knowledge, and all research should be within the tenets of the World Medical Association Declaration of Helsinki (World Medical Association 2013).

There were a number of ethical considerations that were addressed in the study. First, there was the matter of obtaining the informed consent of respondents. It was ensured that each respondent was aware of what the research was about and the extent of their expected participation in the study and each of the respondents signed a consent form (see Appendices 3 and 4). To execute such a process in KSA was very challenging as can be found in more detail in the following sections about 
the process of recruiting participants. Rather than just informing them of the research, the researcher was repeatedly asked to justify the value of the research and provide identification; such issues are less likely to be encountered in the UK. The second ethical concern was the protection of the identity of the respondents. This was very important since it was possible that the respondents could reveal sensitive information regarding their perspectives that may not be popular with their peers or other entities and could place their next visa or registration application in jeopardy. As such, it was important to make sure that they could not be identified as a respondent in the study. To ensure this, no identifying information was asked from the respondents. None of the forms used in the study contained the names of the respondents, their place of work, or any such information. In addition to this, any information in the transcripts that can potentially reveal the identity of a respondent was stricken from the transcript. The signed consent forms contained the only record of participant's identity, and these were stored only to be used in the case of a respondent contesting his or her willingness to participate in the study after having initially agreed to do so. Since they had the right to withdraw from the study at any time, the consent documents were never needed for this purpose. This is of course, essential in any research project. However, in KSA there is a very real possibility that jobs could be lost and lives ruined if this was not carefully managed.

Another important ethical concern was ensuring that no physical or psychological harm can fall upon the respondents as a consequence of participating in this study. The respondents were duly oriented about their rights as respondents. They were 
asked to select the most convenient dates and locations where they would be available for interview. They were also informed that they were under no obligation to answer any of the questions in the interview, and they may decline from answering any question that they did not feel comfortable with. It was important to both operate within research ethics guidelines and also be aware that these would be insufficient to protect participants in Saudi Arabia. Therefore, extra sensitivity and precautions were employed based on the researcher's own knowledge of the organizational issues within KSA.

\subsection{Methods}

Having identified the research design and theoretical framework of this study, this section proceeds to a presentation of the procedures that were undertaken in the conduct of the study. This includes the sampling, data gathering, and data analysis procedures, as well as procedures that were taken in order to ensure the quality of data gathered and address ethical considerations.

\subsubsection{Manchester Metropolitan University Sampling}

The population of interest for this study was that of healthcare professionals in KSA hospitals. In line with the protocols of qualitative design as detailed in Merriam (2009), purposive sampling was employed to collect a sample from the population. The only inclusion criterion that was used was that the respondent should be working as a healthcare professional, specifically either as a doctor or a nurse, at a hospital in KSA at the time of the study. Participants were selected from hospitals that were known to have palliative care services and hospitals which did not have such services to be able to collect respondents from a diverse sampling frame. 
Specific hospitals were selected for approach due to their reputation if they were known to have palliative care services, plus those added due to the researcher's own personal connection to certain hospitals, which was felt would be an advantage.

Hospitals were visited, and permission sought from the hospital administration to approach potential respondents and ask them if they would like to participate in the study. Following this, potential respondents were approached directly and asked if they were willing to participate in the study. Participant Information Sheets were available in both English and Arabic (see Appendices 5 and 6), and consent was taken with a Participant Consent Form in either English or Arabic (see Appendices 3 and 4). With regard to the desired sample size, a definite sample size was not set in this study since, as explained by Merriam (2009), qualitative studies do not depend on gathering data from a sample of a size that can be considered as statistically representative of the population. Rather, sampling in qualitative design is conducted until data saturation is reached. Data saturation is the situation in which succeeding respondents collected for the sample are found to no longer able to provide anything new to the data that had already been gathered from previous respondents (Merriam, 2009). As such, sampling was continued in the study until data saturation was reached. This differed from sampling techniques employed in many other qualitative studies where sampling was conducted in just one site, and data gathering was repeatedly conducted. This was not an option for in this study since it was felt that multiple interviews may attract too much attention from the 
organizations and there would be a risk of the findings being influenced by the hierarchical nature of the service.

\subsubsection{Data collection}

Semi-structured interviews were used to collect data from the respondents. As discussed by Merriam (2009), semi-structured interviews are ideal data gathering instruments for qualitative research since they allow a level of flexibility that cannot be achieved using survey questionnaires or objective measurements. In a semistructured interview, a set of core questions is developed which directly addresses each of the research objectives of the study. In this study, the core questions focused on the following concerns. First, respondents' understanding of the definition of palliative care was established. Second, the core questions focused on respondents' ideas about the importance of palliative care in their work setting. Third, the core questions examined respondents' inputs regarding the relevance of palliative care as a speciality. Finally, the core questions considered respondents' inputs regarding the progress of palliative care as a speciality at their work settings, and what developments can be made in this area of healthcare.

Following each core question, follow up questions were determined and asked based on the answer of the respondent to the core question. Follow-up questions were asked to clarify an aspect of the respondent's answer, have the respondent explain an aspect further, or focus on a specific part of the respondent's answer. Further follow-up questions were asked until all issues concerning the core question were addressed. All of the interviews were conducted one-on-one with each of the respondents and were scheduled according to the respondents' 
specifications in terms of time and place. The interviews were transcribed verbatim and stored in an electronic database. The core questions that were used for the interviews are enumerated as follows.

1. Can you give a brief description of your work responsibilities?

2. What is your understanding of palliative care?

3. Have you ever experienced palliative care for a family member?

4. Have they been looked after?

5. How is palliative care handled?

6. What role does faith or spirituality play in the patient's and/or family's beliefs and what influence do they have on behaviour?

7. What role does the spiritual provider play on the dying patient?

8. In what way do you think that spiritual care is applied?

9. How do you deal with the cultural differences?

10. Can you tell me about pain medication (Morphine)?

11. How do you deal when diagnosing the patient with, for example, cancer?

12. What are the palliative organisations (association)?

13. What are the legislation and constitution regarding palliative care in KSA?

14. What role do media play in palliative care?

15. What do you think about careers in palliative care?

16. What can you say about people who specialize or want to specialize in palliative care? 
17. How do you perceive palliative care as a specialization for a nurse or physician?

18. Would you consider specializing in palliative care?

19. Are these nurses specially trained for palliative care?

20. Are there any training opportunities provided to hospital staff on palliative care?

21. How developed would you say palliative care is in your hospital?

22. Are there any specific issues with regard to palliative care in your hospital?

23. Do you have any other comments on the issue of palliative care?

24. Do you have any suggestion or recommendations for the future?

25. Would you advise and recommend that palliative care as an essential measure in all hospitals?

26. Is there anything else you wish to add?

27. Are you happy with the interview? (This was the final question in each of the interviews).

Please see Appendices seven and eight for a complete copy of the interview schedule in both English and Arabic.

\subsubsection{Data Analysis}

Data in the study was analyzed using the five steps for thematic analysis as detailed in Braun and Clarke (2006). Further information on the conduct of each step was sourced from the works of Merriam (2009). As such, the steps that were finally 
used in the conduct of the study which included elements from both Braun and Clarke (2006) and Merriam (2009) are described in detail as follows.

Step 1: Reading and re-reading to familiarise oneself with the data

The major aspect that separates qualitative research designs from quantitative research designs is the former's reliance on the researcher to serve as the tool for data analysis. In qualitative research, the researcher is expected to examine data and draw out inferences from the data without the aid of numeric estimation. This means that it is essential for the researcher to be acutely familiar with the data gathered, which can be achieved through sufficient exposure with the data. As such, the first step in thematic analysis requires the researcher to read and re-read each interview transcript that was collected in the study. Through this step, the researcher is expected to be able to know the general content of each transcript and be mentally stimulated by this content enough to be able to return to any content of interest later in the analysis process.

\section{Step 2: Coding Data}

Once the researcher has become sufficiently familiar with the data, the next step proceeds towards breaking down the data from each transcript into its basic components. In this regard, Braun and Clarke (2006) defined a code as a group of text that conveys some relevant information. A code, as clarified by Merriam (2009), need not be a complete sentence. Rather, what is important is for the researcher to understand the relevance of a code to at least one of the objectives of the researcher's study. In this step, the researcher carefully reads text from a transcript and identifies each code that is present in the text. In this study, 
highlighters were used to code the transcripts, and each code was linked to the original participants, in order to explore all of the existing perspectives that were offered by the different respondents. This ensured that the codes were properly documented, ensuring that the researcher can go back to any code and identify which transcript it originated from.

\section{Step 3: Collating codes into axes}

After each transcript is coded, the next step is for the researcher to categorize each code under different axes. Braun and Clarke (2006) defined an axis a point of interest in the study for which there may be different perspectives. In this study, an example of an axis is "conditions nearing death," which refers to anything that has to do with a patient's conditions nearing death. There can be a wide range of codes that are relevant to this axis, such as codes from a participant's perspective about how important comfort is as a patient nears his or her death. These codes are thus placed under this axis. Since there are no predetermined axes at the start of this step, the researcher is tasked with formulating axes as needed. A code that falls under an existing axis is categorized under it, but a code that does not fit into any existing axis needs to be categorized under a new axis. As discussed by Merriam (2009), it is important for this categorization to be conducted one transcript at a time due to the fluid nature of thematic analysis. New axis may be constructed with each new code, and a fresh transcript can enable a researcher to use the axes that were constructed from the previous transcript. 


\section{Step 4: Reviewing and Refining Axes}

Once all of the transcripts have been categorized into axes, the analysis proceeds to an iterative process of reduction, where codes under each axis are reviewed and based on this review, some axes are decomposed while others are collapsed. Decomposing an axis means splitting it into more than one axis. This happens when the codes within the axis are so broad that it is possible to focus on two entirely different matters of interest in the axis. This denotes that there are actually two functioning axes that are served by the codes categorized under that axis instead of just one. On the other hand, it is also possible for there to be a common axis that is being served by codes from more than one axis. In this situation, the two axes are collapsed to form a single axis. This process is conducted iteratively on the data until no further refinements can be made.

\section{Step 5: Naming and Defining Themes}

Finally, after refining each axis, the analysis process turns towards identifying themes from each axis based on the codes that were categorized under it. A theme is defined by Braun and Clarke (2006) as a common viewpoint about a matter of interest in the study that was expressed by various participants at various opportunities throughout the course of data gathering. This stage of analysis equivalent to the final stage in Corbin and Strauss' method, and consisted of examining the themes that were identified under each research objective, and considering the relationships of those themes with one another in order to be able to come up with the grounded theory that explains the phenomenon of interest of this study. That is, from the themes that were identified in the previous stage, 
theories were developed as to why palliative care in KSA had not been developing very well. In this stage of the analysis, the researcher is expected to fulfil the role of a reflective analyst, and it is assumed that it is not possible to undertake this stage without incorporating the subjective perspective of the researcher. However, the researcher is expected to keep this subjective perspective in check and to cite it in the development of the theory. The validity of the analysis depends on the ability of the respondent to identify the most reasonable themes and develop the most logical theory that can be drawn based on these themes.

In order to strengthen the validity of the analysis, the analysis process for four of the interviews was repeated by my supervisors. Both PhD prepared nurses, one has worked in several areas of nursing and is an experienced qualitative researcher. The other is an experienced research methodology nurse. The themes that were drawn from this repetition were compared with those drawn from this $\mathrm{PhD}$ researcher's original analysis. This served as a way to check if the themes that were drawn were replicable. After comparison, it was clear that the themes from both researcher and the supervisors were consistent in meaning, albeit not totally consistent in words used.

\subsubsection{Interpretation and translation issues}

The interviews were conducted in Arabic and English according to the preference of the interviewee, with seven participants completing the interview in Arabic and three participants (one American, one Filipina, and one Saudi) completing the interview in English. Once the interview transcription was completed, the researcher translated the Arabic transcripts into English in preparation for analysis. 
The nature of the translation has an impact on the outcomes, and it was essential to ensure that the English translation passed on the same significance as the statements in the Arabic form. Firstly, another reverse translation from English into Arabic was done and contrasted with the first Arabic version. This purpose of 'backtranslation' is to confirm that the meaning in the content is the same in both Arabic and English languages. This gives a sign of reliability of the target language rendition. Once this was complete the researcher compared between the English and Arabic language transcriptions.

These methods helped to ensure consistency between the English interviews and the translated Arabic interviews. However, it is not possible to iron out all discrepancies. There is a noteworthy inconsistency between how the two languages operate and it was difficult to translate precisely from one language to the next, particularly if there was no comparable importance or word representation relating to the syntactic and grammatical style of the Arabic language. This is most easily demonstrated in the use of 'sayings', which are often senseless when translated into another language. Below is an example of one such saying, its literal translation and then its meaning translation:

Arabic phrase: يضرب أخماساً لأسداس

Literal translation: Set forth fives for sixes

Alternative meaning: beating around the bush

Other researchers in similar situations have found that the differences in the languages did not affect the comprehensive analysis and results of their studies. For 
example, (Twinn, 1997)did not find a major impact on the analysis and findings while making the translation from Chinese to English. Interpretation of the meaning was more important than the translation of each word individually. Therefore, participants' understanding and the translation of that were considered more important than just a literal translation. When comparing the final translated text, the translation of the participant's meaning from Arabic into English gave almost the same meaning.

\subsubsection{Trustworthiness of the data}

Measures were developed in order to ensure the trustworthiness of collected data in the actual study. First, the potential respondents were provided with every opportunity possible to decline participation in the study. It was made clear to each respondent what the study was about and it was explained that participation was completely voluntary, so that only respondents who were actually willing to share their inputs on the study topic would be included. These people were assumed to be less likely to lie about their actual perspectives with regard to the issues of interest in the study. Second, in conducting the interviews with respondents, they were encouraged to be as frank as possible with their responses, and they were assured that their inputs would remain anonymous. The researcher also attempted to establish a good rapport with each respondent, to make them comfortable and trusting in sharing their inputs during the interview. It was explained to respondents that there are no right or wrong answers to the questions, but rather, what was important is that they can share their positions on each matter truthfully. As a further safeguard of the trustworthiness of the data, iterative questioning was 
employed in the conduct of each interview. The researcher periodically went back in order to clarify different points that respondents made in previous questions, in order to check his own understanding of the answers given and to ensure that the respondents' answers remained consistent throughout the interviews. Any inconsistency detected, however, was not pointed out to the interviewee to avoid any confrontational incidents. Rather, these were noted in the collection and analysis of the data.

\subsection{Ensuring quality in Qualitative Research}

Qualitative research does not have the same established conventions for ensuring quality as are found in quantitative research, yet it is of utmost importance to ensure quality. Quantitative research is judged reliable, referring to the potential for the research to be replicated and produce the same results, and validity, referring to whether the research measures what it proposes to measure (Merriam, 2009). The same quality indicators cannot be used for qualitative research. To illustrate, a sample size large enough to be considered representative, which is a marker of quality in quantitative research, would not be appropriate for qualitative research as it would be too large to allow in-depth analysis.

Some researchers have called for clear criteria for judging the quality of qualitative research, but others see this is neither possible nor desirable (Buchanan 1992). For example, Sandelowski et al. (1997) argue that quality in qualitative research cannot be determined by following set criteria, as qualitative researchers from different disciplines and theoretical backgrounds may have different criteria for assessing the quality of a study. In 2015, Sandelowski furthered this argument to suggest that 
despite the numerous checklists which have emerged with the intention of allowing evaluation of qualitative research, they are framed in language that does not allow for discernment in judging the value of qualitative research. Murphy et al. (1998)suggest asking general questions of qualitative research to assess quality without applying specific criteria which would be too narrow to apply to all qualitative studies. This idea has been developed by Treharne \& Riggs (2014)who suggest evaluating qualitative research in accordance with broad guiding principles. They suggest an overarching theme of transparency, that is, being clear about how the research has been undertaken at all stages, with a further three general principles: personal reflexivity and user involvement; transferability; and triangulation of data.

Transparency involves having an audit trail of the research process and a clear representation of the analysis stage, including attention to cases which do not fit into the themes and theories developed from the majority of cases.

Personal reflexivity includes evidence of extended engagement with the subject matter and sensitivity to context and reflecting upon one's own role in research. End user involvement includes ascertaining whether participants understand the findings to be true to their experiences.

Transferability requires the analysis to have reached a level of abstraction, and triangulation is the use of more than one method of obtaining data to increase reliability. 
These broad principles can be seen to incorporate the earlier work of key authors in this area(Lincoln and Guba, 1985; Pope and Mays, 1996; Yardley, 2000; Spencer et al., 2003; Tracey, 2010), as to appropriate matters to consider in order to judge the quality of qualitative research. Lincoln and Guba (1985) is the most highly cited system of quality for qualitative research.

A meta-narrative of sources explicitly addressing quality in qualitative research was undertaken by (Reynolds et al., 2011). They found two strands of this subject, one addressing quality in the process of qualitative research, and another addressing quality in outputs. They concluded that these two strands: "should be brought together into a flexible framework to help qualitative researchers to define, apply and demonstrate principles of quality in their research." (p1).

This PhD research has aimed for quality in both practice and output, within the practical constraints of the situation. Transparency has been achieved through practising reflexivity, that is, reflecting on and acknowledging my role in the research, both in the undertaking and the reporting of the research. End user involvement was not a specific aim of this project, due to time and resource constraints. However, the design of the interviews was such that those to whom the research is relevant had the opportunity to contribute and the iterative design of the interviews allowed for checking that my understanding was congruent with their intended message. This was achieved by checking at the end of the interview to ensure that they didn't want to change anything. Another strategy which could have been used was to return interviews to respondents for reviewing and confirmation. This was considered but disregarded, partly because it was felt that 
there might be internal or external pressure on the respondent to change what they said, even if it were still 'true', but also because of the possibility of transcripts being intercepted, which could cause problems for the interviewees.

Within the context of palliative care in KSA, giving those involved in palliative care a voice can be seen as a key progression within the wider context of further research and improvements in this area.

The data collection and analysis was undertaken in such a way that it may be possible to apply the findings from this study to KSA as a whole, therefore suggesting transferability. Triangulation was not an aim of this study, due to time and resource constraints.

\subsection{Conducting qualitative research in the KSA}

There are a number of challenges that were faced in conducting qualitative research in the KSA. Firstly, the KSA has a very different cultural environment to the UK, and in fact, its culture is unique within the world. As such, undertaking research in KSA posed unique challenges, and part of reflexivity and transparency is to reflect on and be open about the challenges faced and their potential impact on the research. Comparing the UK to KSA may help to illuminate some of the issues faced.

In the UK, people are generally trusting of researchers, for example, they believe that you are who you say you are and if you say that their information will be kept confidential, they trust that this is the case. This is not so in KSA. People may suspect that you are connected to the secret police, and many ordinary people have the habit of not disclosing more information than necessary, just in case it 
may be used against them in future. This is due to corruption which is unfortunately within the system of healthcare. This corruption is well reported in the media (e.g. (Trenwith, 2015). This means that doing research in KSA is uniquely challenging, especially in utilizing a qualitative design where in depth perspectives of stakeholders are needed for data gathering. The typical mistrust that people in the country have can prevent them from sharing vital information to the study. However, the researcher's position as a native, who is known, and whose family is known within the healthcare system in KSA, is an advantage. Due to this, he was able to leverage that advantage by making participants more at ease about sharing their views and confident that their contribution would not be used in any way to cause them harm. This situation also heightens the value of this research, which was completed despite many challenges, and provides an insight into an almost hidden phenomenon. This suspicion is particularly the case with a qualitative approach, which asks people to express their views, which may be seen as a risk.

Recruiting participants was an area where particular challenges were faced, which were unique to KSA and illustrate the cultural environment in which the research took place. For this reason, a personal account of the researcher's experience of recruiting was kept, in diary form, from each of the hospitals involved in the research. Reflection upon some of the issues that are raised by this and their potential impact on the research follows.

Due to an unreliable postal system and weak infrastructure, participants were not recruited via letter or email, but by my visiting the hospital. This decision was also made because of the suspicion that people can have in KSA towards people 
undertaking research, and it was felt the research could explain better in person. Due to having limited time, the researcher went to the hospitals without prior appointment, as making an appointment can take a long time. This is usually ethically unacceptable, and it is more usual to ask potential respondents to 'opt in' to the research. However, the nature of 'KSA makes this an impossible strategy. To advertise and be open about recruiting would have been to put pressure on anyone who was interested in taking part and would mean that if anyone tried to 'opt in' everyone would know about it, so anonymity could not be controlled.

Suspicion by 'gatekeepers' was a potential obstacle in getting to speak to the relevant people. For example, some people may have suspected that the researcher was from the $\mathrm{MOH}$. On the other hand, having a letter of permission to conduct the study helped. The suspicion about research, which was, in fact, more evident in expats than Saudi nationals, was summed up by one non-native physician "we eat from paradise of KSA, and you Uncle Fahed want to lead us to 100 issues".

Status in an important element when dealing with people of authority. Status is partly conveyed by image, and therefore clothes worn to the appointment had to be carefully considered. For example, to wear the national dress in a casual fashion denotes status. Lineage is also an important element of Saudi social structure, and therefore the researcher's relationship to his father, who was known within one of the hospitals, was beneficial and mentioning his nameput people at ease who were initially suspicious about the study.

Other interviewees were nervous of the researcher's perceived authority, and the presence of the tape recorder, however explaining the purpose of the research put 
them at ease. One potential participant, who ultimately did not participate, was possibly embarrassed by his own lack of knowledge about palliative care and was slightly aggressive in his response.

Saudi hospitals have a multi-national workforce, and there are some tensions between different nationalities at times, and this also played out in my attempts to recruit participants.

\subsection{Summary}

This chapter has revisited the research aims and described the method by which these aims are sought to be achieved, from a theoretical perspective. The researchers own role within the research has been explained, and how this influenced the aims of the study and the design of the research.

In summary, this chapter describes a pragmatic methodology, drawing on Grounded Theory and Symbolic Interactionism, leading to a qualitative methodology using thematic analysis to analyze the data. The cultural environment within which the research takes place is discussed, and its impact on the process and the final value of the research is considered. Finally, the methods used to achieve the aims of the research have been reflected upon and evaluated.

The next chapter will present the findings, based on analysis of the interviews with participants. 


\section{Findings}

The cultural context of KSA is known to be different from the West, where the concept of palliative care originated and where recent progress in palliative care is most evident (Hui et al., 2010). This study sought to investigate the practice of palliative care within the cultural context of the KSA. In particular, this study looked at how palliative care is incorporated by healthcare professionals in the Kingdom of KSA. The purpose of this approach was to produce an account of the perceptions of KSA hospital nurses, physicians, and healthcare administrators regarding palliative care; to determine if physicians, nurses and healthcare administrators in KSA are aware of palliative care, engage in palliative care practice, and recognize the value of palliative care and palliative care nurses; and to identify issues that KSA hospital nurses, physicians, and healthcare administrators have regarding palliative care. To this end, semi-structured qualitative interviews were undertaken with ten currently practising healthcare providers in KSA.

Research participants' responses shed light on the previously mentioned issues. In particular, research participants' definitions of palliative care; their perceptions of palliative care as a medical specialisation; their statements about the role of the family and the role of the media in palliative care. In addition, their explanations of the issues and problems interview participants faced in providing palliative care in their hospitals, all shed light on these research objectives. A discussion of the research findings as they relate to these objectives follows. This is preceded by a visual representation of the themes and subthemes discussed, the purpose of which is to orient the reader and can be referred to while reading the findings. This 
chapter also provides 'pen portraits' of the participants, which gives the reader important information to reference while reading the results. It would perhaps have been fruitful to have included more detail about the respondents, as this would have benefited the understanding of their comments, however, it was decided that to provide more information may jeopardise confidentiality.

\subsection{Pen portraits of participants $(n=10)$}

Ahmad is in his thirties. He works as a physician in a city hospital with a total capacity of 1095 beds. He is native to KSA.

Chokri is in his fifties. He works as a physician in a 936-bed tertiary care facility in a city. He is native to KSA.

Hailey is in her fifties. She works as a nurse in a 690-bed facility in a city. She is from a developed country with good palliative care provision.

Gabir is in his forties. He works as a physician in a 690-bed facility in a city. He is a native to KSA.

Farah is in her fifties. She works as a physician in a 690-bed facility in a city. She is from an Arab country.

Gail is in her forties. She works as a nurse in a rural hospital with 30 beds. She is from a developing country with little palliative care provision.

Ihsan is in his fifties. He works as a physician in a rural hospital with 30 beds. He is from an Arab country.

Bandar is in his forties. He works as a physician in a rural hospital with 30 beds. He is from an Arab country.

Dakheel is in his fifties. He works as a physician in a rural hospital with 30 beds. He is from an Arab country.

Emad is in his forties. He works as a physician in a rural hospital with 30 beds. He is from an Arab country. 


\subsection{Outline of the themes and subthemes}

Table two provides an outline of the themes and subthemes that arose during the research and which are discussed in more detail throughout this chapter.

Table 2 : Outline of themes and subthemes

\begin{tabular}{|c|c|}
\hline Theme & Subthemes \\
\hline Defining palliative care & $\begin{array}{l}\text { Patient } \\
\text { Family } \\
\text { Incurable illness } \\
\text { Death, dying and the end of Life } \\
\text { Holistic view }\end{array}$ \\
\hline $\begin{array}{l}\text { Perceptions of PC as a } \\
\text { specialism }\end{array}$ & $\begin{array}{l}\text { Unique } \\
\text { Growing } \\
\text { Need and importance }\end{array}$ \\
\hline Doctors and Nurses & $\begin{array}{l}\text { Perceptions of PC } \\
\text { Palliative care as a team } \\
\text { Personal qualities }\end{array}$ \\
\hline Issue and problems & $\begin{array}{l}\text { Communication } \\
\text { Lack of resources } \\
\text { Lack of human resources }\end{array}$ \\
\hline $\begin{array}{l}\text { Knowledge, training and } \\
\text { education }\end{array}$ & $\begin{array}{l}\text { Need for PC education } \\
\text { Wider context }\end{array}$ \\
\hline Role of the media & $\begin{array}{l}\text { Important role } \\
\text { No role } \\
\text { Negative role }\end{array}$ \\
\hline Medication & $\begin{array}{l}\text { Availability } \\
\text { Procedures } \\
\text { Issues }\end{array}$ \\
\hline Legislation and policy & $\begin{array}{l}\text { Legislation } \\
\text { Policy }\end{array}$ \\
\hline Culture & $\begin{array}{l}\text { Truth telling } \\
\text { Respecting culture } \\
\text { Role of religion }\end{array}$ \\
\hline Future & $\begin{array}{l}\text { Vision } \\
\text { Needs }\end{array}$ \\
\hline
\end{tabular}




\subsection{Defining Palliative Care}

When research participants were asked to define palliative care, several subthemes emerge from their responses. Not surprisingly, the main subtheme which emerges is the focus on the patient in palliative care. Other subthemes are the focus on not only the patient but also for the patient's family and the reference to incurable illnesses. That is, research participants recognize that palliative care involves caring for patients with illnesses for which there is no curative treatment. This subtheme leads into the next subtheme which is that of references to death, dying and end of life. As research participants note, because Palliative Care deals with patients with incurable illnesses, death is often a part of the job. A final subtheme which emerges from the data is that of a holistic view. Many research participants recognize that palliative care provides a holistic care of the patient. In particular, many participants emphasize that palliative care not only involves providing physical care of a patient but social, spiritual and psychological care as well. The following is an in-depth discussion of the subthemes which emerge from within the theme of participants' definitions of palliative care. The only participant who had not previously heard of the term palliative care was Gail.

\subsubsection{Focus on the patient}

One of the patterns which emerge from the data is that palliative care is focused on the patient. In fact, almost all of the research participants (9 out of 10) reference the patient in giving their definitions of palliative care. For example, in providing his definition of palliative care, Ahmad, a palliative consultant interviewed on January 
22,2014 , simply states: "The definition of palliative care is looking after a patient that has incurable, chronic illness". It is important to note that Gail, a nurse supervisor interviewed on January 7, 2014, was the only participant who did not mention the patient in providing her definition of palliative care; she admitted that she did not have prior knowledge of the speciality before the interview took place.

\subsubsection{Family}

A significant theme which arose in this study was the importance of providing palliative care not only for patients suffering incurable illnesses but also for their families. In their definitions of palliative care, four out of the ten research participants reference the family, in addition to the patient. For example, Hailey, a Western palliative nurse interviewed on January 22, 2014, includes the patient's family in her definition of palliative care, which she suggests is: "...to improve the quality of life and well-being of individual patients and their family care givers." Gabir, a palliative consultant, interviewed on January 20,2014, expands on this inclusion of the family in providing his definition of palliative care, which suggests how the family is included: "The main concept of palliative care is about the quality of life, which means when somebody is having life-threatening illness, so palliative care is covering the quality of life either related to patients and their families." In defining palliative care, Chokri notes: “...there is a known definition for the palliative care over the world, it's the WHO definition, but generally, it is a holistic specialty, which focuses on a certain type of patient and their family members, so it's not only about the patient, but also his family members, because they also share in the suffering." Ahmad defines palliative care as providing support not only for the 
patient but "for the family as well." Chokri elaborates on this and further includes the family: "It [palliative care] is a holistic speciality, which focuses on certain types of patients and their family members, so it is not only the patient but also his family members because they also share the suffering." Finally, Gabir describes how the holistic perspective is adapted to the family: "The main concern is about the quality of life, the symptoms management and also dealing with patients' families in comprehensive ways, physical, psychological, social and spiritual issues". Overall, the family is seen as an important part of providing palliative care to patients suffering terminal illnesses. Participants recognized the importance of communicating with and supporting patients' families through the difficult process of losing a family member, as well as in providing for their spiritual, psychological, physical and emotional needs. In particular, many participants reference the importance of spiritual services for the family members of patients. For example, as Chokri explains: "Also the spiritual specialist may help the patient and the family on certain type of matters regarding their current situation, like how to pray about the current situation and when the patient loses some physical abilities and also gives advice about dying and how to react to it and so on." In addition to spiritual services, communication with families of patients is seen as important. As Ahmad simply states: "Communication is very important for the patient and the family members; it's very important."

The other participants did not mention the family in their definition. This does not necessarily imply that they do not feel that the family is an important element of palliative care, but that they were emphasising other points at this time. For 
example, Emad states: "Palliative care includes all the patients who suffer types of disease that cannot be cured". However, it is possible that those participants who did not mention family had limited definitions.

\subsubsection{Incurable illness}

Another important subtheme which emerges from the data regarding patients' definitions of palliative care is the focus on caring for patients with incurable illnesses. Six of the ten research participants note incurable illnesses when providing their definitions of palliative care. For example, as Emad, a Syrian physician interviewed on January 6, 2014 notes: "Palliative care includes all the patients who suffer types of diseases that cannot be cured, chronic diseases, and giving those patients a cure or sedative to stop the pain of the patient, since the disease is incurable, so death could happen at any moment."

In addition to mentioning 'incurable illness' in general in their definitions of palliative care, two of the research participants mention the diseases of cancer in particular. For example, in defining palliative care Bandar, an emergency physician from Syria interviewed on January 9, 2014, states: “...palliative treatment is for the chronic diseases like cancer."

Other participants did not specifically mention incurable illness, either because their definition was wider than this, for example, Farah thought that palliative care was given to a patient whether their illness was incurable or not, or their definition was narrower, for example, Ihsan mentioned that palliative care was only given to bedridden patients. 


\subsubsection{References to death, dying and end of life}

Another subtheme which emerges from the data on interview participant's definitions of palliative care is that of references to death, dying and end of life. Five out of the ten participants reference these terms when providing their definitions of palliative care. For example, as Chokri notes: "...Palliative Care is focused on certain types of patients, patients with incurable or advance diseases which mostly lead to death." Similarly, Ihsan, a physician interviewed on January 9, 2014, states that: "...palliative treatment as we know it here is to be applied to the bedridden patients, or the patients who are not improving in their health cases, like the patients of cerebral stroke, so those types of patient will not improve so we will give them palliative care till the end of his life."

Hailey's definition was most accurate, taking into account that palliative care should be introduced early in the disease trajectory, "it's a holistic approach to not simply end of life, not in the terminus days but from the time of diagnosis really if there are issues to be addressed that would help to improve the quality of life." Gabir also made a similar statement. While other participants did not specifically mention death or end of life, their definitions can be seen to include this phase of illness. Bandar, Gail and Ahmed specifically mentioned the chronic nature of the illness, emphasising this over death and dying.

In summary, half of the participants believed death, dying and end of life were important aspects of palliative care. However, while the remaining five participants did not include these specifically, their comments showed this phase of the illness was included in their care and concept of palliative care. 


\subsubsection{Holistic view}

A final subtheme which emerges from research participant's definitions of palliative care regards the holistic focus of the speciality. Four out of ten participants mentioned the holistic approach in their definitions. For example, as Ahmad notes: "...Palliative Care is a holistic approach..." Hailey's definition further defines the holistic approach of palliative care when she suggests that: "... whether it is physical, psychological, emotional, social, spiritual; it is [palliative care] a holistic approach."

All of the participants who defined palliative care as holistic in nature mentioned the psychological aspect in particular, but only one participant included psychological care in his definition. For example, while Bandar did not note the holistic nature of palliative care, he did emphasize psychological care: "[palliative care] includes the improvement of the psychological state of the patient...".

The participants who did not refer to the holistic nature of palliative care were focused on the role of palliative care in relieving pain and other physical symptoms.

\subsection{Summary of 'defining palliative care'.}

In reviewing the theme of research participant's definitions of palliative care, several subthemes emerge. These subthemes include: the focus on the patient and his or her family; caring for patients with incurable illnesses; references to death, dying and end of life; and finally, the holistic nature of palliative care with an emphasis on providing not only physical care but also social, spiritual and psychological care in particular. 


\subsection{Perceptions of Palliative Care as a Medical Specialisation}

One of the goals of this study was to produce an account of the perceptions of KSA hospital nurses, physicians, and healthcare administrators regarding specialisation into palliative care. Towards this goal, research participants were asked about their perceptions of palliative care as a specialisation. Several subthemes emerged from this theme. In particular, many of the research participants perceived the discipline of palliative care as a unique or rare within the healthcare field. In addition to noting the uniqueness of palliative care as a medical healthcare speciality, interview participants also noted that palliative care is a growing speciality in need not only in KSA but in the rest of the world. While noting the anticipated worldwide growth of palliative care, research participants recognize the need and importance of palliative care as a specialisation. In particular, research participants described the need for palliative care in terms of how many patients they have in need of palliative care in their respective hospitals. They also noted the increased need for palliative care by noting the increasing age range of patients and therefore the increase of diseases in need of palliative care treatment. Despite this perceived need, participants noted there are few palliative care specialists in the Kingdom. This is especially unfortunate as research participants noted the specific need for spiritual care as well as the potential benefits palliative care specialists propose for helping to increase the quality of care in hospitals as well as reducing costs and the workload of other healthcare practitioners. A discussion of research participants' perceptions of palliative care as a specialisation follows. 


\subsubsection{Palliative care as a unique or rare specialisation}

When asked about palliative care as a specialisation, many of the research participants perceived the discipline as unique or rare. In fact, four out of the ten research participants describe the discipline's rareness or uniqueness when asked about their perceptions of palliative care as a specialisation. Surprisingly, the comments describing the uniqueness of palliative care as a speciality were equally distributed among positive and negative comments.

In positive terms, regarding the uniqueness of palliative care as a medical specialisation, Gabir simply states: “...it's a beautiful specialization, a very rare one and serves a large segment of the society." The rareness of the speciality is further described by Emad who adds that this rareness is a benefit: "it [palliative care] is a rare specialization, so as long as it's a rare specialization, you get more benefits."

Another way in which participants describe palliative care as a unique speciality in positive terms regards how it deals with easing the suffering of patients in pain. For example, as Chokri explains his perception of palliative care as a speciality: “... I think it's a unique field, and those who work in the palliative care field should be proud of themselves, especially when we take a look at all the suffering of patients who need this field all around the world for ages. So they should be proud, and they should look at themselves as heroes who made an effort to downsize the pain of those patients who has been suffering for ages around the world."

In addition to those research participants who describe the uniqueness of palliative care in positive terms, three participants note that this uniqueness can also be negative. For example, Ahmad describes the ways in which the uniqueness of 
palliative care as a speciality can also be a detriment. In particular, the way in which the uniqueness of palliative care, when compared to other disciplines, makes it difficult to secure funding: "But you know, palliative care... is not like other specialities. Other specialities, like neurosurgery, or other surgical specialities, where there is funding from... private organizations. They're all funded. So funding is difficult to know in our field."

In addition, Chokri who also notes palliative care as a "unique career" describes how this can be quite negative. He notes that "...there are no encouraging incentives or bonuses, especially when we know that the impression about the palliative care field among the healthcare professionals is that it is a hard and tough field and needs a lot of the psychological effort from the specialist in the field". He continues explaining the necessity of providing financial incentives in order to encourage individuals to specialise in palliative care: "...if you want to encourage people to participate in the field, you have to reach them and convince them about the importance of the field, also by paying them well bonuses to encourage them...". Finally, he explains how this lack of incentives translates to a shortage of palliative care specialists: "...the number of palliative care specialists is so low, around all the world, not only here, where here is much lower than the world, we have a lack of palliative care specialist, even for doctors, or nurses or any part of the team of palliative care".

Despite his positive comments mentioned earlier, Gabir expresses similar sentiments regarding the ways in which palliative care as a unique specialisation can be a negative thing. In particular, he describes how its uniqueness makes it 
difficult to recruit potential palliative care specialists: "It's a rare specialization, so recruiting specialized people in the medical, nursing and in all multidisciplinary teams it is very difficult. It is less difficult for the doctors, but with the nursing and other specialities, it becomes harder and harder".

\subsubsection{As a growing speciality in demand}

In addition to noting the uniqueness of palliative care as a speciality, interview participants also noted that palliative care is a growing speciality in need not only in KSA but in the rest of the world. For example, when asked about his perception of the decision to specialise in palliative care, Ahmad notes: "I think it's a great idea. It's very big opportunity to have... and there's a lot of room to grow in palliative care. All over the world, not only in KSA...". He continues explaining the worldwide need for palliative care: "And it's a global thing, and there's a need everywhere you go, okay. Because I feel palliative patients are lost, between they don't know where to go; they're bouncing from one doctor to doctor. I think there the approach, and the thing they need is not there, and the best thing is with palliative care. So, I think it has a, it's a great opportunity, not only for physicians, like yourself, nursing, many team approaches, other healthcare professionals. It's very important, and it has a lot of growth."

\subsubsection{The need and importance of palliative care}

While noting the anticipated worldwide growth of palliative care as a speciality, research participants also recognize the need and importance of palliative care as a specialisation. Even Bandar, who first learned of palliative care as a speciality during the interview process notes the importance of the discipline: "As I told you before, 
it is my first time hearing of this speciality, but it is beautiful, with a big role, especially for patients with chronic diseases". Research participants describe the need for palliative care in terms of how many patients they have in need of palliative care in their respective hospitals. They also note the increasing numbers of patients in need of palliative care. Despite this perceived need, as participants note, there are few palliative care specialists in the Kingdom. In addition, not everyone values this speciality. This is especially unfortunate as research participants note the specific need for spiritual care as well as the potential benefits palliative care specialists propose for helping to reduce the workload of other healthcare practitioners.

When describing the need for palliative care, some research participants explained this need in terms of how many patients they have in need of palliative care in their respective hospitals. For example, Ihsan notes the number of patients in need of palliative care just in his small hospital. "It's a very good field, and it will be great to specialize in this field, and I wish to see it in all hospitals, not just the big ones. As I told you, we have ten palliative care patients although we are a small hospital, so of course, the big hospitals with 1,000 beds will have many palliative care patients". Dakheel expresses a similar sentiment when he explains the need for palliative care in his hospital in terms of the number of patients who need this care: "I have in the ICU and the CC almost $70 \%$ or $80 \%$ of patients who are always in need of palliative care. Thus ICU is always busy $100 \%$. I have patients who stayed and occupied places at the ICU for two and three months. I have one patient who dwells for five years in the hospital. If there is an independent palliative care unit that can take 
independent care of such cases, it will give a chance for treating another patient".

Dakheel further explains the increased need for palliative care by noting the increasing age range of patients and therefore the increase of diseases in need of palliative care treatment: "We have a large percentage [of patients in need of palliative care], and it is rising, the reason is the evolution in all fields of health sectors, which increases people's ages. Now you can see people 99 and 100 years old that come to the hospital with different types of diseases. The percentage of patients aged 100 years, for example, has increased about 50". He continues explaining the increased need for palliative care: "The development of science and treatments and diagnostics has increased proportion of humans' age and thus increased diseases, cancer, strokes and others. And increased the proportion of people who need palliative care, but unfortunately, there is no specialization in palliative care. We have a significant proportion of patients in need of palliative care".

As Dakheel's comments above illustrate, the developments in other areas of medicine have increased the human lifespan with the unfortunate consequence of increasing instances of diseases associated with age such as cancer and strokes. This increase in age and the diseases associated with old age have indubitably increased the need for palliative care. Unfortunately, as Dakheel notes, this increase in the need for palliative care has not been met in the Kingdom. There remains a lack of palliative care specialists in all areas. This lack of palliative care specialists is mirrored in comments by other research participants. 
Similar to the sentiments expressed by Dakheel, as Bandar explains, despite the increasing need, there are few palliative care specialists in the Kingdom: "as I said, it's a great specialization, and there is not much of it in KSA here, there is no palliative care specialist in the whole north area."

In addition to these negative comments, as Hailey notes not everyone recognizes the need and importance of palliative care. This can have very real effects on the availability of funding. "Palliative care isn't trendy. Palliative care isn't as some physicians would put it, it's not sexy, it's not you know, it's not like heart transplant or conjoined twins, or you know, it's...it's end of life and it doesn't really carry much meaning until it impacts you, one of your close relatives or friends is suffering, vomiting intractably or many other things that go towards end of life. Then they are reaching out help me! Help me! But for many in the upper echelons with the greatest respect to them, they don't value palliative care and so the resources for physician education, for nursing education, the community education, for supporting home health palliative care in the community, it's not there." The lack of palliative care specialists in the Kingdom as well as the lack of recognition of the need and importance of palliative care is particularly troubling when viewed in relation to research participants' comments regarding the specific needs for spiritual care as well as the potential benefits of having palliative care specialists within hospitals all over the Kingdom.

Emad notes a specific palliative care need for patients is spiritual care when he says: "There must be difference in tasks and work. We need doctors also we palliative 
care specialists. Most of the patients now need spiritual peace, that's all they need, so palliative care is important, so l empower people to go for this specialization".

In addition to illustrating the specific needs for spiritual care, research participants also note the potential benefits of having palliative care specialists within hospitals all over the Kingdom. For example, as Gabir notes: "I can estimate that $40 \%$ of the patients in our hospital need palliative care and palliative care is not only for cancer patients, all patients need it, even the healthcare professionals from the others specializations realize the important of the palliative care. Palliative care is good for the whole institution because it gives good quality of care and reduces the cost. So palliative care has a great future in KSA, and there will be a great demand for it". He continues, noting: "It is a very accurate specialization, and it is so important to be professional in this discipline because a lot of physicians and nurses if they are not specialized in it, they cannot deal and treat the patient in the ideal way and they will face a severe problem in treating the patient... if you are not specialized in the field you will affect the healthcare of the patient, and you will face a lot of problems, maybe you would feel depressed, and you will be so tired of dealing with these difficult cases. But if you are specialized and you are adapted to such things, this will help you a lot as a nurse or as a doctor, and even the quality of care will be way better, the cost of the hospital will be less".

Gail expresses similar sentiments. In particular, she notes how an increase of palliative care specialists in hospitals will help to decrease the workload of other healthcare practitioners. "Palliative care consists of supportive care... It is to comfort the patient, support the family, and then attend to the needs of the patient. So if we 
have a palliative person, it will be less work for other nurses and other professions. Because one person will deal with palliative care only. He will focus only on palliative care for the patient".

\subsection{Summary of palliative care as a medical speciality}

In reviewing the theme of research participant's perceptions of palliative care as a medical specialisation, several subthemes emerge. These subthemes include the rareness or uniqueness of palliative care when compared to other medical healthcare specialities (which can be both positive and negative), and that palliative care is a growing speciality in demand not only in KSA but in the rest of the world. While noting the anticipated worldwide growth of palliative care as a speciality, research participants also recognize the need and importance of palliative care as a specialisation. However, despite this recognized growth of, and need for, palliative care participants note a lack of palliative care specialists in the world and especially in the Kingdom. In addition, they note that not everyone recognizes the value and importance of palliative care. This is especially unfortunate as research participants note the specific need for spiritual care as well as the potential benefits palliative care specialists propose for helping to reduce the workload of other healthcare practitioners.

\subsection{Doctors and nurses}

This theme looks specifically at the role of doctors and nurses in palliative care, firstly looking at the perceptions of doctors and nurses towards palliative care, then considering the teamwork element which emerged as significant. Finally, the sub- 
theme of the personal qualities of doctors and nurses and how this impacts upon their ability to provide palliative care is discussed.

\subsubsection{Perceptions of palliative care}

It was agreed upon by participants that palliative care is not well understood by most doctors and nurses in KSA. Ahmed explains, "I used to think palliative care was only patients that were dying at the spot, you just see the patient dying...you just...you were having a patient dying every second or every day, so that was my concept. And I still think many physicians have this concept; many nursing have this concept, and many healthcare professionals have this concept".

There is a dire need for more doctors and nurses specialised or trained in palliative care, but in order to attract doctors and nurses to palliative care, they first need to understand what it is, "I think the people have to know what is palliative care, and we have to promote this specialty through our...medical students, residents, and this will attract people to come to your specialty, and nursing as well... But, if you stay aside and closed, I think this will cause not people come to the specialty, not knowing what's happening, and therefore you will not grow your task, your force, your...manpower, and then you will run into burnout...your nursing, your physicians will burn out, and this will be an issue for you" (Ahmed).

\subsubsection{Palliative care as team}

Both Chokri and Hailey mentioned the teamwork element of palliative care, implying that for effective palliative care, doctors, nurses and other professions are required to establish the team, "The other concept is teamwork, since it's holistic, and you are taking all the sight views, then you will need an expert team, the 
doctor, the nurse, the social specialist, the spiritual specialist, the physical therapist and so on" (Chokri).

\subsubsection{Personal qualities}

Due to the nature of palliative care, but more specifically in KSA due to the lack of organized palliative care services, the quality of end of life care will depend upon the personal qualities of the doctors and nurses. Both Bandar and Emad describe palliative care in their institution as "personal effort by the doctors" (Emad). This issue was specifically raised by half of the participants. Hailey describes how the spiritual element of palliative care comes from the compassion of certain staff, as opposed to specific provision, "I think spiritual care comes in this hospital more through the compassion of some of the staff...umm not religious leaders, not...not umm sheikhs or spiritual...but people who have compassion that's...there showing actions and their words and their expression and their body language... Umm, you know how some nurses and physicians and others can walk in a room and know the patient's in pain without speaking a word".

Other skills mentioned include the ability to communicate with patients of all cultures and education levels "Of course we should be at all levels, for the welleducated patients and non-educated also, the doctor should be smart enough to reach all levels, sometimes we find it hard to deal with the well-educated patients and their families, because they already know the side effects and symptoms, and so on, they have issues and concerns about morphine and so on, but at final, as I said the doctor should reach all the levels" (Farah). 
The consequences for patients if the medical staff are not confident in communicating can be far reaching, "some are quite comfortable talking with patients about the goals of care, what do you want to achieve? ...sitting down with a patient face to face and with the family members and explaining and giving those options...Some physicians they can't even say that the cancer has reached an advanced stage, they can't even tell the patients that there's a possibility they won't survive past six months. Some physicians keep pumping chemotherapy into those patients...they don't give the patient an option so instead of stopping the chemotherapy, the futile medication, sitting down with the patient and talking with them and giving them the option to go home with the care and support they need. They'll keep them in the hospital; they'll die in the hospital uhh a miserable death" (Hailey).

Gail sees palliative care as one of the day-to-day duties of nursing and describes how she and other nurses manage this, "For the people, it is their choice to enter this. So let's says about me I chose nursing instead of pharmacy. So my choice to give care to my patients and my family...So as far as I know, I talked to other nurses, because we are living in one hostel. So after duty, we will talk about "How's your patient? Like this like that. So how did you take care of, how about this?", every shift we will talk about our patient. Like sharing, sharing our care, our duty during that time." (Gail)

Conversely, Hailey mentions personal qualities which may be detrimental to providing good palliative care, "different backgrounds where the doctor is God, can do no wrong. You have a physician come from Cairo; he's king of the world, he just 
knows everything and whatever he says...and I'm not saying Cairo; it could be anywhere. Umm, they believe they're infallible and no one should question them whether it's a nurse or a patient. The attitude is gradually diminishing, but it's still uhh it gives a sense of power to people who otherwise wouldn't have power, and I think that can really seriously get in the way of...of...of a the truth telling the patients (Hailey).

\subsection{Summary of 'doctors and nurses'}

This theme emphasises the need for training in palliative care, which is generally thought to be not well understood among doctors and nurses. Palliative care is seen as highly reliant on teamwork, and also the personal qualities of doctors and nurses involved.

\subsection{Issues and Problems in Palliative Care}

In describing the issues and problems with providing palliative care in their hospitals, interview participants describe a range of challenges. The main issues and problems, according to interview participants are communication difficulties and a lack of resources.

It is important to note; of all of the interview participants, only Ihsan indicates that there have been no issues about palliative care in his hospital. When asked if there were ever issues with regards to palliative care he claims: "Never before, because there is good care here, so even if the patient passed they will not make issues. So for my last three years since I am here, I have never heard of an issue here." A discussion of the main issues and problems in providing palliative care in KSA follows. 


\subsubsection{Communication}

One of the biggest issue interview participants describe are problems with communication. Interview participants describe a range of communication problems, including communication issues in general, but also specific issues with language. Bandar explains communication issues in general at his hospital:

"Sometimes the problem isn't about the patient or his family, sometimes it's about the guests of the patients, it's an issue, because sometimes, all the guests want to ask the doctor about the situation and want to know about the disease in details, and they come one by one, which will consume the doctor's time of course... what should happen, is that the doctor should talk only to one member of the family, but everyone wants to ask the doctor, they don't ask other members but only the doctor".

\subsubsection{Language barriers}

In addition to describing issues in communication in general, interview participants also describe the specific issue of language barriers at their hospital. For example, as Bandar simply states: "...most of the trouble we face is because of language differences." He continues to explain these troubles: "...this is a problem we always face, not only the culture difference, but also the language. They say that if you can talk one language then you have one culture but if you can talk two or three languages, then you have many cultures. We suffer at communicating with some patients, Arabian cultures -Syrian, Saudi, Egyptians, Sudanese- we don't have a problem with it, but the problem comes with communicating with non-Arabs, or people who do not know the Arabic language. Unfortunately, most of the staff here 
are not Arabs, so this is a real problem." As Bandar notes, the multi-linguistic situation of KSA presents unique difficulties in communicating with non-Arab patients who do not speak the Arabic language.

Gabir further explains the specific challenge language barriers propose in providing palliative care: "The great challenge is for nurses, because 99\% of them they do not speak Arabic, and dealing with palliative care at the end of the life, communication is so important for it, and they don't understand the culture of the patients, so we have a language barrier and a cultural barrier, and it is very important for us."

\subsubsection{Lack of Resources}

Another major issue KSA healthcare providers face in providing quality palliative care to their patients is a lack of resources. Many of the interview participants describe a lack of resources which impede them from providing the best palliative care possible in their respective hospitals. In particular, they describe a shortage of human resources such as a shortage of palliative care physicians and nurses trained in palliative care, as well as a lack of spiritual care specialists. The following is a detailed discussion of these resource issues.

\section{- Lack of human resources}

One of the specific issues within the lack of resources concerns a lack of human resources. As Chokri notes: "...the most important issue is the staffing because the need is there." He continues, detailing the issue of shortage of staff and the effect that it has on care in the hospital: "...you do not have a psychologist, you do not have a spiritual care provider, a social worker specialist in the palliative care team, and you do not have this and that and so on. You do not see a social worker in the 
whole hospital spend his time with his patients. Then this results in superficial work; he is covering here and there."

\section{- Shortage of palliative care physicians}

Chokri is not the only research participant who notes the issue of shortage of staff. Similarly, Ahmad notes the shortage of palliative care physicians in particular when he says: "In KSA, there are about ten palliative care physicians. We have about 25 million approximately; we have more now. So, if you do the math, one palliative physician is taking care of about two and a half...about more than two million right? Which is a lot. If you go to Europe, a palliative care physician covers about 250 thousand, which is less, okay, so we have a big responsibility here. So, inshallah (God willing), it will improve inshallah (God willing)." Ahmad's comparison of the amount of palliative care physicians in KSA when compared to in Europe, about 100 times more palliative care physicians in Europe per person than in KSA, illustrates the shortage of palliative care physicians in the Kingdom.

\section{- Shortage of nurses trained in palliative care}

Another issue interview participants indicated is the shortage of nurses trained in palliative care. For example, as Gabir notes: “...unfortunately, we do not have any nurse who is trained in the palliative care, we have a great shortage of nurses trained in palliative care, and that is the main challenge that we are facing."

\section{- Lack of spiritual care specialists}

Another area of shortage in human resources indicated by research participants is the lack of spiritual care specialists. Chokri discusses this issue in the context of his 
hospital and compares this situation to other hospitals in the area: "Actually here in the hospital, we are suffering from lack of a spiritual care specialists, and this lack is very high. For some time before, we were in a very good situation, and we had someone who used to play this role, but they left the hospital, as you know, everyone is trying to get a better place and career to work on, and unfortunately since not a short time we are asking and requesting the administration to help us on this side, but until now we are suffering that there is no one taking this role, the spiritual care I mean. The other hospitals around us have a better situation, like the military hospital and King Fahad Medical city they are in a better situation than us, especially in this part."

\subsubsection{Not a lack of resources, but of poor allocation}

One of the more interesting comments by a research participant when asked about the issues in the hospital they work at was Hailey's. When asked whether there is a lack of resources at her hospital, Hailey gives a profound answer that it is not a lack of resources per say, but rather the allocation of resources: "Well, there shouldn't be, that's all I can say that there is more than enough here to go around and then some. It's...it's the distribution of resources that's the issue in my opinion. It's the equitable distribution of resources. It's inequitable, and it's the...creaming off the top for certain...you know you get money from the government, a certain budget every year. Automatically some... the cream is taken off the top for various pet projects or whatever it's taken for. By the time it gets to something like palliative care, it's not there, but as far as resources as I say this hospital...the opportunities are fantastic. Umm, we need to look at our business model, we really need to look 
at how this new hospital should be run, more effectively, how we can provide care for our patients in a more uhh sustainable and compassionate way; and hopefully that will come, but no we do not lack resources. There's enough money to go around the world ten times. It's just a question of where... where's that money located that's the key."

\subsection{Summary of 'Issues and Problems'}

Providing palliative care in KSA is not without its issues or problems. As interview participants indicate, two of the major issues involve communication (language barriers in particular) as well as facing a lack of resources (human resources in particular). As the interview participants' statements indicate, these issues and problems impede Saudi healthcare practitioners from providing the highest quality palliative care possible. These results can be tied into the wider constructs of family and culture. The following diagram describes the relationships between Culture, Family, and the Palliative Care Professional in examining challenges that face palliative care development in the KSA. 


\section{Palliative Care Challenges}

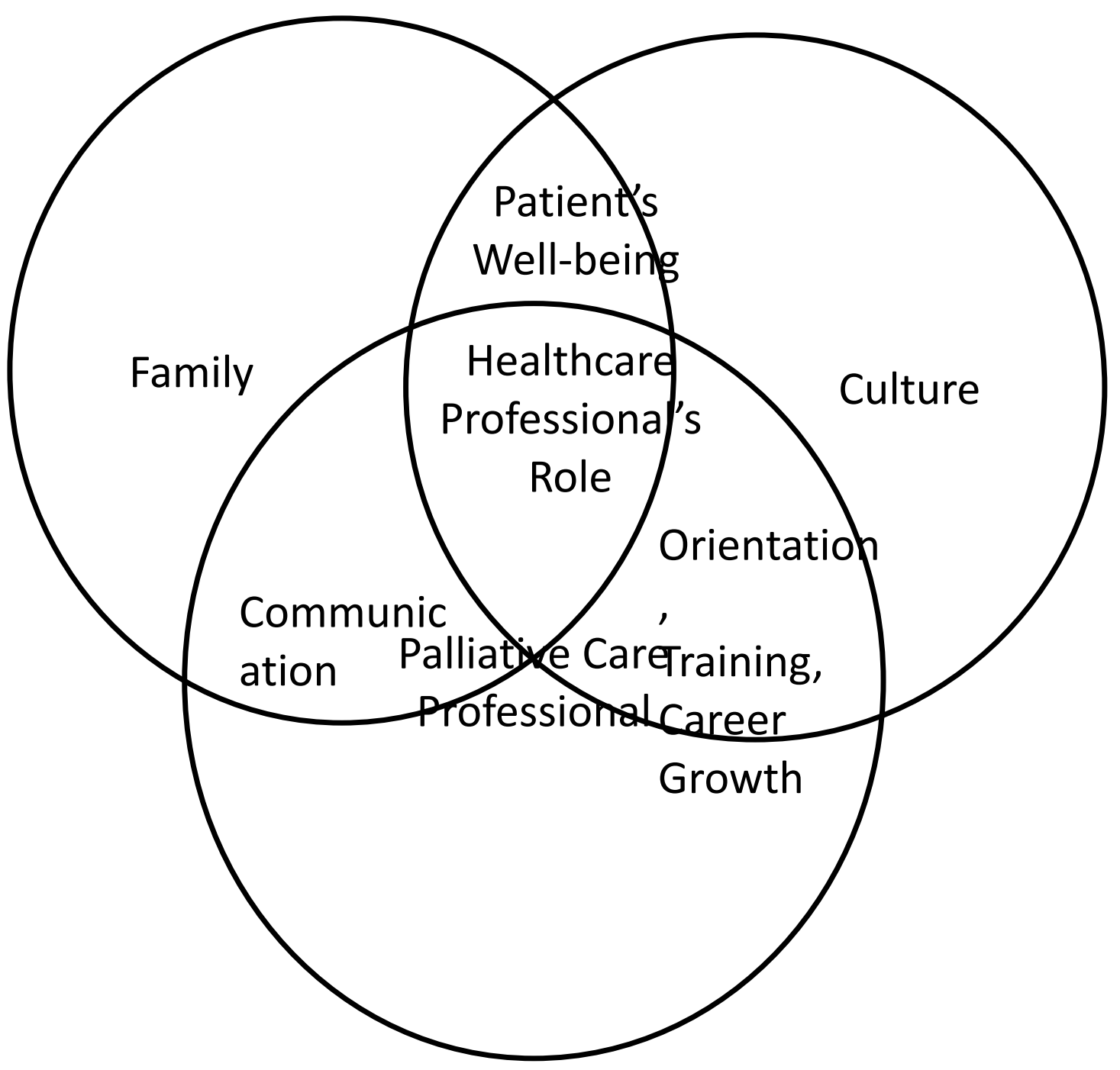

Fig 1: Palliative Care Challenges

As shown from the diagram, family and culture are intertwined in terms of two aspects, which are the patient's well-being and the healthcare professional's role.

Results from the interviews were able to establish that the family in the KSA plays a significant role in determining the direction of care of the patient. From interviews with healthcare professionals, they discussed how they valued not just the wellbeing of the patient, but also the peace of mind of the family, and that the close 
connection between patients and their respective families is a strong cultural attribute in the KSA. In line with this, interviewees expressed the challenges that they faced in interacting with the families of terminally-ill patients, particularly when the family does not perceive palliative care practices as necessary or even in the best interest of their relative. This led to the identification of weaknesses in the training of healthcare professionals in terms of developing communication skills that can help them better explain palliative care benefits to the families of their patients. As discussed by some interviewees, language barriers exist which keep them from being able to explain the benefits of palliative care as thoroughly as they want to their patient's families. On the other hand, some expressed how their limited background in palliative care also hindered them from being able to discuss such matters more effectively with patients or patients' families. In relation to this, the interviewees also expressed the challenge of there not being enough opportunities for training and professional development in palliative care in the KSA context; considering the specialisation to be undervalued in the local industry. Without these opportunities, healthcare professionals do not see sufficient incentive to actually pursue this specialization in their careers.

\subsection{Knowledge, training and education}

This theme explores the participant's views in reference to knowledge, training and education, which was acknowledged as a need by all interviewees. Participants also provided information about current educational and training interventions. Another sub-theme that emerged was the need to not only educate healthcare providers, 
but also to educate the family of those receiving palliative care, and the general public.

\subsubsection{Need for palliative care education}

The participants were unanimous in their observation that KSA lacks people well educated in the field of palliative care, with a perception that there are more such people in the larger hospitals, "so maybe in the big hospitals, they are more educated" (Gail). While the area is growing, "the awareness of palliative care is in our hospitals it's growing among physicians" (Ahmed), "there are still no nurses specialised in palliative care" (Dakheel). Hailey suggests that the principles of palliative care are not well understood and that this problem may be exacerbated by the multi-nationality of the workforce, which meant that some staff might have trained in countries where the concept of palliative care is not well understood.

Strong opinions were expressed about the need for palliative care education and how this should be done. Several respondents suggested a two-level education initiative, whereby palliative care is developed as a specialism, through undergraduate courses and being included in rotations, combined with a move to provide a general education to all health professionals on the basic principles of palliative care, particularly nurses "it needs to be out there in the community, in the primary healthcare facilities at a general level where they understand pain and symptom management, not in great depth but it's...it's there, it's available, there is knowledge, and that's not happening" (Hailey). The specialists would be able to disseminate information about palliative care to improve the general level of awareness. Chokri suggests on the job training, "so you sometimes need to make 
compromise, you take people who have got the interest to work in this field and you train them while they are working".

Nurses were cited as being central to palliative care, "nurse must be the one to know how because palliative care is all about nursing care mostly" (Ihsan). Ahmed cites his experience of working overseas with a nurse practitioner trained in palliative care. Due to this experience, Ahmed recognised the importance of nursing in palliative care and has hopes that there will be training for nurses in KSA in the future. On a local level, Ahmed describes how they have trained a nurse informally in palliative care, "One of the nurses we trained our self, and..he attends with us lectures as well, with the physicians. He's..he doesn't have specific training in palliative care, but he has...he's using oncology, nursing oncology..so we trained him..on the clinic, in patients, educating the patients on giving them..taking medication."

Palliative training for nurses was unanimously noted to be particularly lacking, "unfortunately, we don't have any nurse who is trained in the palliative care, we have a great shortage in the trained nurses in the palliative care, and that's the main challenge that we are facing, cause we are dealing with nurses who has no subspecialties or training, and it's a great challenge honestly, in KSA generally" (Gabir).

Gail, a nurse supervisor, while explaining that palliative care is an integral part of nursing, accepts the need for specific training in palliative care, "everywhere you will go, you are supporting patient, so palliative care goes with our jobs so I cannot 
say that there is a development...but somehow but it also depends on staffing that's why I am suggesting there will be a course for palliative care better because they can focus on that, and then they can have more information regarding palliative care but for us it will...we will just insert it in our job...the government should provide one course to study is better so we can provide palliative care to the patients and then somehow the patient will be relieved" (Gail).

\subsubsection{Current progress}

Ahmed discusses progress within his own institution with regards to palliative care education, "we started our training here. We have a fellowship program; I'm currently the program director...our plan is to train more physicians. Maybe they can go outside of Riyadh; they can start their own programs, and this will spark up a collaboration and have more physicians being all over the kingdom inshallah (God willing). So this is our..our vision in palliative care". Ahmed also discusses a planned project to train nurses in palliative care and explains the aims of this training, "we want them to look out to assist pain better; how to look at the comfort of the patient more better; how to recognize imminent death, patients dying".

Chokri says that his colleagues are in the process of establishing a training course for nurses. However, Gabir notes issues at an organisational level with providing such training, "we tried to provide once but we faced a lot of difficulties to find training programs outside, and we are supporting and send the people but the nurses face problem in the acceptance in the programs, I noticed this thing" (Gabir). Another issue is that one-off training courses are not sufficient to affect real change. Both Hailey and Ahmed describe how one-off courses only have a short 
term effect, and that skills learnt are quickly lost without continual reinforcement. For palliative care to see significant improvements, training and the consequent awareness needs to become "part of the fabric" (Hailey).

Gabir and Farah both specifically cited improvements in palliative care in their experiences. This may be related to their own specific experiences, for example, Gabirs' experience seems to be much more positive than other participants, "but now people know about palliative care. There are a lot of academic activities have been done, as physicians, in the ER, in the IC, and everyone demands it. In the level of the awareness, there's a great development, and it's not only in the hospital but also in the whole world in the last ten years... The people started to know what's palliative care and what's the importance of the palliative care and I think still we are new born subspecialty we need time to have more specialized people". In contrast Farah cites a personal experience of a close cousin dying, at which time she was a doctor but had no education in palliative care, whereas working in NGHA had changed her perception "the patient died by the cancer pain, and the pain wasn't well controlled, after I came here, I knew that the patient must be pain free, symptoms free, and to die comfortable. My view changed 180 degrees after I came here before and after I work here".

\subsubsection{Wider context}

Hailey suggested that resources were lacking for palliative care, as it was not a priority for policy makers, "They've done it all for us, we've got it, we've got the knowledge and we're building on that knowledge but here in Saudi [pause] We're not using it, why? I don't know. So umm we've got a lot of work to do. I don't know which 
would be first, education I guess would be educating physicians but we've got to educate the policy makers and decision makers. We need someone who's... who's as I say who's out there and very popular with the public, who's got the political will, who's got the money who can take it forward" (Hailey).

While the participants would often refer the 'the West' as having a more advanced system of palliative care, Ahmed's experience of working in Western countries was that many people working on palliative care teams had only a minimum amount of training in palliative care. Hailey corroborates this observation with her own experience, "one of the issues I see with palliative care at this hospital but at also many other hospitals not just in Saudi...is that nurses are called palliative nurses because they work as part of a team. They have not trained to use their critical thinking skills; they're not trained to use their critical judgment, they're not trained to properly assess patients whether it's for pain, symptoms, symptom management".

\subsubsection{Educating others}

Ihsan, Ahmed and Gail mentioned the need to educate not only health professionals but also the patient and the family, in palliative care. Ahmed talks about educating the family in the concept of palliative care, "Even the family members when you tell them "palliative care", you have to tell them what's palliative care. They still don't know what's palliative care. But, once they understand the concept, they really appreciate what they're getting".

Emad discusses from a more general point of view how health education is lacking in KSA, "Bedouin community, the health education is bad, they believe in some kind 
of cures like the herbal treatments, they believe it will help and cure the patient, but in fact It won't, it's just additional cost and efforts, but because of the low education and ignorance they believe it will help, so as I said the health culture is low here... the country is Bedouin, and there is low health culture here". (Emad)

Gail suggests giving "health teachings" to the patients, and Ihsan focuses on the practicalities of caring, "So the family must be cultured to deal with the patient about repositioning and when to tell the doctor when there is red spots, for example, not to leave the area wet around the patient, telling them all needed info. Some of the families here took the suction device, and they now know how to use it, so the idea is to culture the family" (Ihsan).

\subsection{Summary of 'knowledge, training and education'}

This theme suggests that the lack of education and training in palliative care is clearly understood and that some progress is being made within KSA in this regard. However, progress is slow, and some suggestions are given as to why this might be, including that it is not a priority for policy makers. Participants also advocate educated the public about palliative care, which may help palliative care be implemented at an earlier point in the disease trajectory.

\subsection{The Role of Media in Palliative Care}

There appears to be some disagreement among the participants regarding the role of media in palliative care. While two of the ten participants cite media as having an important role in palliative care, another five of the participants define media as having little to no role, and a further three participants state that the media has had a negative role. This may be partly due to the different ways in which participants 
interpreted the question. Some focused on publicity by reputable information givers, and some focused on personal media such as Facebook.

\subsubsection{Media as having an important role}

Only two out of the ten participants interviewed state that media plays an important role in palliative care. In particular, Ahmad describes the media as playing "...a very important role". He continues, describing a recent event at his hospital: "We had an awareness day a couple of months ago. We had media involved in this, our media involved, and we had interviews, people went out, a lot of physicians went on the media and gave a lot of interviews and educated the community and public about palliative care". He continues "...There is an interview on a famous channel, of one of our physicians, talking about the awareness day in palliative care [and] another on national TV."

Gabir's statement about the role of media expresses similar sentiment: "Mass media has a great role; first of all, it increases the public's awareness of what palliative care is, even for the healthcare professional so that they know what palliative care is and what it is we are doing about palliative care, so I think it's important".

These two statements by interview participants illustrate that media may have an important role in promoting community awareness of Palliative Care and the job of the palliative care physician. 


\subsubsection{Media as having no role}

Half of the ten interview participants define media as having no role. In particular, these participants note that they have not seen media play a role at their hospital. For example, Farah, a palliative physician interviewed on January 20, 2014, explains the role of media at her hospital: "They [the media] don't have a great role here, and they have never talked about it while I have been here." In addition, Hailey explains how media has not played an important role in palliative care at her hospital either: "I've never seen anything, whether it's on these electronic boards or... the only time that I've seen end of life care sort of advertise per say is when this physician Frank Ferris came from the states, from California for a workshop. I think he's been here on two occasions but actually anything to do with end of life care, death and dying...no, I've never seen anything".

The previous statements by Farah and Hailey illustrate that in many hospitals, the media plays no role in palliative care. However, although some of the research participants describe palliative care as having no role, they also emphasize that it is important for the media to play a greater role. For example, Ihsan explains the need for media to play a role in palliative care in the community: "Since most families have a palliative care patient, the media should play a role in culturing and educating the families, so that people get more awareness about palliative care...". Hailey expresses a similar sentiment and explains her wishes for the role of media in palliative care: "I've never seen anything. I would love to see, I would love us to have some open forums, I would love us to have some groups, I would love family members to be able to come in groups and sit and share with... whether it's 
[hesitation] television station or radio or whatever just to share...to share the burden, but we're not there yet".

\subsubsection{Media as having a negative role}

Three of the research participants believe media plays a negative role in palliative care. For example, as Dakheel, a Syrian medical director and physician explains:

"There is no role for media; instead it [media] is working to tear down everything we have built in the hospital, especially the internet. It is mostly done by social media like Facebook. They spread rumours that no one accepted the patient because he or she is in the dying stage". He continues by explaining the negative way media's role affects the public's perception of palliative care: "...this affects the relation and the faith between the patients and the hospital and the $\mathrm{MOH}$ as a whole and whoever did that must be punished".

As the previous statements by interview participants indicate, there are divergent views regarding the role of media in palliative care in hospitals and communities.

\subsection{Summary of 'the role of media'}

It is clear that the participants interpreted the word 'media' in different ways, as some focused their answers on reputable information givers, such as news agencies, and other focused on personal media, such as Facebook. The findings suggest that the perception of the media's role is largely dependent on the personal experiences of the individual participants. It certainly seems clear that the media could play a bigger role in educating the public about palliative care, and it may be that those involved in palliative care need to find constructive ways of harnessing the media. 


\subsection{Medication}

When research participants were asked about medications in their hospitals, several subthemes emerge from their responses. One of the main subthemes which emerged is the focus on the availability of medications. Another subtheme which emerges from the data is the reference to procedures for administering medications. A final subtheme which emerges from the data is that of issues with medications. In particular research participants mention issues with patient misuse of medication, cultural issues with medications, as well as knowledge issues surrounding medications. The following is an in-depth discussion of the subthemes which emerge from within the theme of participants' responses regarding the topic of medication in their hospitals.

\subsubsection{Availability}

One of the subthemes which emerge from the data on medication is that of availability. In fact, seven out of ten of participants reference availability in their explanations of medication at their hospitals. All but one of these seven research participants note that their hospitals do not face a lack of medication at their hospitals. For example, as Chokri simple explains: "It's available, here we are not facing a problem of lack of medication, and that's a very good feature in our hospital". Ahmad expresses similar sentiments when he addresses the availability of medications at his hospital, opioids in particular: "...opioids here are well accessed. We use opioids frequently... most of the opioids are available here, and... initially when we started, it was kind of difficult in prescribing Morphine. Now, it's better than before, through a systematic way. Most of the physicians can prescribe 
opioids, but I think we still have to work in the community how to prescribe opioids. Still, there are some prescriptions, but in the hospital, we have no problems in our Hospital Institute. And I think most big hospitals in Riyadh don't have a problem". Gabir's response expands upon the topic of medication access and describes all the medications available at his hospital: "For the access of the medication here in Alharas hospital, which is the one of the reference hospitals in KSA, we almost have the all pain medication, Morphine, Oxycodone, Methadone, Hydrocodone, Codeine and Hydromorphone, so almost we have all of pain medications, and we do not face a problem in the medication access".

In addition to these descriptions of the availability of medications, Gail theorizes why medications are so widely available in KSA, in particular, due to the riches as a result of the oil industry: "So far I can say about Rafha, government they are supportive, really, the main beneficiary of ...especially oil is ...this is a rich country because of oil, Sah! (right)? So their main number one beneficiary is health and second I think is education. So as for us, we are here in Rafha, we have advance supportive machines and advanced technology... It is oversupply... really! Oversupply compared to our Philippines. In there we are Meskeen ( a poor country), so they just supply oversupply medicine and everything. So just imagine on the expiry date, we should throw it for their own benefit of the patient. They are thinking about the patient's safety."

It is important to note, only Emad notes a shortage of medication in his hospital: "We don't have anything other but morphine. Other hospitals have morphine oral, its better, also we use SubQ and IV, but for chronic disease, the patient should use 
orals, but we don't have oral morphine, and the injections of morphine are hard for the patient daily. Sometimes we give the patient other cures like non-steroids, but for morphine, we have injection morphine IV and IM and SubQ".

\subsubsection{Procedure}

Another important subtheme which emerges from the data regarding medication is that of the procedures surrounding its administration. In particular, research participants describe the procedures for choosing which medications to use, how medications are administered, as well as how much medication is administered.

Research participants describe the procedures used in their respective hospitals for choosing which medications to administer to their patients. For example, as Bandar explains addictive medications such as morphine are given as a last attempt at killing pain: "We start with the ordinary painkillers, those who don't cause addiction, if a patient came in and asked and insisted the nurse to give him morphine or any type of these medication, the nurse will give him the medication last. That's why we higher the clearance level of giving these medications to the consultant and specialists only, and that minimized the usage". Similarly, Ihsan explains the procedures for how pain medications are administered in his hospital and how doctors and nurses are involved: "Morphine and painkillers are available here in the hospital, and we have a policy here in the hospital about morphine as a drug, and it must be given after doctor signature, and there must be a person with the nurse to check that the nurse did the injection in the right way and there we write that the certain patient took morphine. We apply the policy of the drug medication". 
Finally, research participants' comments also address the procedures for how practitioners decide how much medicine to give a patient. In particular, Ihsan explains the orderly procedure his hospital uses for deciding how much medication to give a patient: "Here we have something called 'pain indicator' for all patients not for palliative care patient only, which gives score from 1-10, if the indicator read more that 3-4 doctor will give the painkiller to the patient, and there is a check certain time according to the painkiller given to the patient to see if the pain has gone or no, and it's all written that the patient had morphine or whatever through the certain way and so on. So the pain estimation is important, and it's in our policy, and we give it a well care."

\subsubsection{Out of area}

An issue which arose regarding procedures was how practitioners deal with patients who are discharged from the hospital and require medicine but live far enough away from the hospital as to make it difficult to travel to get their medication. As research participants explain, palliative care patients are given either a month or two of their medications to take home with them. For example, Ahmad explains the procedure at his hospital: "...we give them [patients] the prescriptions for two months. And sometimes the family members are around in Riyadh, and he can take it and go there. Or they can... this is basically what we do. But sometimes our patients don't have a long prognosis. So if they discharge, they have a month or two; so they have a family member that's here, we prescribe, we usually give them two months like if they wanna go home, uhh, if they're outside Riyadh. Uhh..so basically, if they have a family member, they can come to the clinic, 
and then we can, umm, give them the specific medication, and they can take it if there are any issues". As Ahmad's comment illustrates, the family can also be involved in the process of picking up medications on behalf of the patient. Chokri explains the reasoning behind this policy: "We give them a dose of a month, the policies only give us till one-month maximum, and we give them an appointment for checking, then we renew their dose for another month... we see how hard for the patient to come here monthly, so what happens is we give any of the relatives of the patients the dose from the second time and over. And we renew the dose of the patient".

\subsubsection{Issues}

When research participants were asked about medications in their hospitals, one of the subthemes which emerged from their responses is that of issues with administering medication. In particular, one of the patterns which emerge is the misuse of medication. Another interesting issue which emerged is around cultural ideas about medication. A final issue which emerged from the data is that of knowledge issues. A discussion of the issues with administering medication, as described by research participants, follows.

\section{- Misuse issues}

One of the patterns which emerge from the data on medications in hospitals is that of issues with misuse. Two of the research participants reference misuse issues in giving medications to palliative care patients. For example, as Bandar describes, misuse of medication led to a change in the procedures for administering medications in his hospital: "...unfortunately misuse of the medication led us to 
downsize the use of these medications, we assigned that giving these medication must be approved first by a specialists or consultant, according to the section. That was because of the misuse of the medication". Even in cases where misuse has not previously been experienced, the fear of misuse affects how the medication is administered. Emad describes how administering pain medication, morphine, in particular, is proceeded with caution: "...well at the beginning we use the known types of painkillers, then we use morphine when needed... it works well, but after a while, it turns to addiction". This caution is present even though as he explains, there has never been a case to cause concern in his hospital: "[it] never happened before, but it may happen one day... If the patient took a daily dose of morphine, he will be addicted to morphine, but addiction is better than pain".

\section{- Cultural issues}

In addition to referencing issues with misuse, Gail also notes cultural issues surrounding the theme of medication. In particular, she recalls an experience she had when covering for another nurse on a round of home visits. "One time... when we did a dressing I saw, they are putting some... powder. I ask Baba, 'Baba, what is this, what did you put on the wound?' 'Ahah, that one is good, haza (this) long before we are applying this one. It will heal the wound, it will heal the wound and then it will feel better, and then the wound will grow, and then it totally will heal.' And then I told him, 'Baba, this one powder, this is not medicine!' 'Laa (no)!,' He told me no, he insisted". In this instance, Gail had an issue with a patient's use of traditional medicine versus biomedicine. She continues to explain how due to the culture of the surrounding community, a practitioner must accept the use of other 
types of medications: "He insists, 'No this one is a good one, we've been...Even my grand grand grand great grandfather is doing this one already.' So I cannot just tell him to 'No, remove this one! I have no right. Sah (right)? I cannot suggest that this one is not good. It will not benefit the patient, it will just make more problems to the wound because this is not antibiotic whatsoever. We have this modern technology; we have... all these new machines, equipments, and everything and the medicines also. So we have everything, really. Saudi's more advanced than any other country because they have money to buy everything. All the patients' needs, we are providing them. They are providing us to give it to the patient. So that's one thing. So I cannot just remove the culture, what they grow up in the culture. We cannot just tell them to forget about it. You learn from this thing because now we are modern, and then it will benefit...It will do good to your patient. So that's their culture, that's how they grow up... So, we cannot do anything about it."

\section{- Knowledge issues}

A final pattern which emerges from research participants' discussion of medication in their hospitals regards issues with knowledge. In particular, Chokri explains this issue: "The other side that needs support, the other departments" understanding the nature of the palliative care. One of these departments is pharmacy. You will find a problem, not only with us but for other hospitals. And that with regard to palliative care formulary and the method of use of the medications and dosages and so on. Actually, we try to increase or improve the awareness with pharmacists as much as we can but they get turnover and worker changes so from time to time, we face the 
same problem, that people are unable to know how to use their medications, we still communicate with them, but it is a still an area that needs an improvement."

\subsection{Summary of 'medication'}

The findings suggest that in general, pain killing medicines are available, but the procedures involved in administering medicines may be disadvantaging the patient. These procedures stem in part from instances of misuse of medication. There are also some cultural barriers to the use of painkillers.

\subsection{Legislation and policy}

Participants varied significantly in their awareness of legislation, both local and national, and also of the policies within their own hospitals.

\subsubsection{Legislation}

Awareness of legislation and policy issues relating to palliative care varied widely between participants, and it was the consultants within the larger hospitals who had the most knowledge of this area. Some participants had little or no idea about the legislation regarding palliative care, whereas others had an in depth understanding. For example, Ahmed, a palliative care physician from KFMC showed an awareness of international legislation, "So, uhh, as you know, in WHO, umm..indicates that uhh palliative care is a human right. It has to be provided for all...patients; so, if they require it," and had a more positive perception than other participants of the progress of KSA in regards to palliative care. This may be due to being in a position where he had access to knowledge and experience of new initiatives. 
Many participants were aware of palliative care in KSA being behind other developed countries, and noted this as a failing, "the gap between us and the countries who worked on this field is very big, and even those countries think they should give more attention to these field, and planning to invest more on it...in the future we will find out that we are going back while others are going forward in this important field" (Chokri)

Hailey, a nurse from a western country, spoke with confusion as to why the professionals she knew cared so much about their patients had not been politically motivated in this area, "I know they have the understanding and I know they've got the compassion, why is it they lack the political will?", whereas others, such as Gabir, a native palliative care consultant in one of the larger hospitals, had a broader understanding of the issues, and ideas about the process of change, "I feel like we need time, we need first of all to get people in the hospital to be aware about the palliative care, the authority in the main hospitals then through the Saudi councils adopting the subspecialty as a recognized subspecialty, educating public, and then once we have a lot of ideas in the future, we could govern the governmental rules established, but I think the improvement should start from low limit, from the hospital to the government".

Some participants mentioned the recent launch of a specific organisation for palliative care, though different names were given for this and it is not clear if they refer to the same organisation. Others were certain there was no such organisation, and Hailey doubted that there were enough interested parties to form such an organisation. Ahmed appears to have been involved in the launch of the Palliative 
Care Association, the purpose of which he states is to organise scientific matters and increase public awareness. Chokri and Gabir mention the Saudi Society for Palliative Care as a new initiative. It is not clear whether this is the same organisation. Gabir explains the importance of such an organisation, "First thing is to have formal organization as I said, cares about all the palliative issues in the country and trying to be all on the same page. Different hospitals are working individually so this will be helpful in the academic activities for collaboration; researches for organizing symposia and it would be helpful, establishing guidelines, establishing any protocols, that will be helpful for us and educated people also from others cities".

Many participants mentioned the need for legislation in the area of palliative care but differed in the way they thought this could be brought about. For example, Chokri believed the changes need to come from policy level, "so I wish to see more attention from the health policy makers to give attention to this field and invest more on it, from the training side or resources or awareness or whatever, and if this happens, it will reflect on the attitude on the hospitals, every hospital will try to develop itself and will give importance to the palliative care field, and the location of resources and so on", whereas Gabir felt changes within the hospitals would ultimately influence policy, "we need to get the people and the hospitals firstly aware about the palliative care then go to the government" and felt that the newly formed Saudi Palliative Care Society would assist in this process. Farah noted that policy makers needed to follow up with positive action where good practice was found in hospitals. 
There were some specific needs mentioned, for example, having protocols for resolving ethical dilemmas relating to palliative care, "we don't have ethical reference to solve the problem or even to give guidelines. I will suggest to have a reference in every hospital or in the level of the Saudi Council, a legislative or a reference to solve the ethical problems or provide recommendations on how to solve the ethical dilemma" (Gabir). Another need mentioned was to spread the culture of palliative care, and it was suggested by Ihsan that the ministry provides one or more palliative care specialist for each hospital.

Dakheel bemoaned the fact that the government collect statistics on cancer patients, but do not actually analyse or research the statistics, "why I am suffering doing statics reports, and you come from $\mathrm{MOH}$ from Riyadh by flight $1000 \mathrm{~km}$ away, then you come and sleep in hotels, and you get delegation allowance and then you return back to Riyadh in order to just carry statistics...you should make research, why you do not study the increasing of cancer".

\subsubsection{Policy}

The need for good policies and protocols within hospitals was emphasised by many of physicians interviewed. The purpose of the policies should be to unify practice between hospitals, particularly with the aim of spreading good palliative care from the larger to the smaller hospitals, "there must be one protocol, the patient should have the same care and same reply for his answers here or in the recovery hospitals and the same cure of course" (Ihsan), to provide good pain relief in combination with good tracking mechanisms for medication, and supporting home healthcare with a palliative component, "But to have people in the primary healthcare clinics 
who can counsel, who can assess pain, who can talk to family members, we can avoid a lot of these emergency admissions if we focused on what we need in the community whether its primary healthcare clinics, whether its home care" (Hailey).

Most participants were aware of hospital policy, including the consultants in the larger hospitals, and Gail, a nurse supervisor, "we have policy for end of life, so it depends on the relatives also we have this DNR (do not resuscitate). We will ask the relatives about it", though some participants, such as Emad, did not feel policies were clear, "I don't have certain idea or a copy of it, in the western world the laws allow you to put the patient off the ventilator for example if it's a hopeless case, but here, the laws don't give you this right". Other also mentioned a lack of policy in important areas, "We don't have discharge planning, terrible omission in this hospital, in any tertiary care facility. We don't have formalized, strong, discharge planning." (Hailey)

Examples of clear hospital policies included policies about pain and drug administration, and the patient's right to know. There was a perception of the presence policies being associated with 'good' palliative care, "we had set up protocols for these procedures, so we, I can say, palliative care is going well here" (Ihsan). It is interesting to note the contrast of the perception of Ihsan, to that of Bandar, employed within the same hospital, "No there isn't certain policies about palliative care". This suggests a difference in perception of what a palliative care policy is, or that not all staff are equally aware of the policy. 
It was particularly interesting to explore how these policies related to practice. For example, where there was a lack of policy or hospital policy was felt to be detrimental, staff would work around the policy to ensure maximum patient benefit, "the policies do not allow us to transfer them, and there are patients who need palliative care ...but we cannot take them according to our policies. But within the oncology we are understanding each other's according to certain criteria and policies, for example if the patient is no more active management, with no code, we take him" (Farah).

One area of policy mentioned was the patient's right to information. Dakheel explains how despite his awareness of the policy, he does not comply with it, as he feels it is not in the best interests of the patient, "We are passionate people not like western. Thus the emotional and psychological does impact the patients' health, although it is our policy that the patient knows everything about his condition and medication and results and has the right to accept or reject these medications... But tell them that these are last days of his/her illness will adversely affect his mental state". Ihsan has a more positive view on practising that particular policy, "it's his right, and we can tell him in a nice way and give him the hope".

Hailey explains how important it is that staff follow through with hospital policies, "so palliative care can be a truly wonderful, wonderful service or it can be very superficial pretty much in name only". 


\subsection{Summary of 'legislation and policy'}

Despite the divergence in knowledge levels of legislations and policy, the needs for government policies to spread good practice, and for policies and protocols to exist, was clearly expressed.

\subsection{Culture}

Participants spoke about the specific cultural context of their work, and from this emerged the sub-themes: truth telling; respecting culture; and role of religion.

\subsubsection{Truth telling}

Ahmed, Chokri and Gabir each mentioned the issue of 'truth-telling' regarding diagnosis and prognosis when dealing and cancer patients and their families. They all mention the same issue, which is that the family will want to conceal the diagnosis from the patient, for fear that it will lead to loss of hope which will impact negatively on their emotional state. The participants are aware that this is contradictory to the rights of the patient, which is to be informed of their health status, but Ahmed points out that this desire for the family to hide the diagnosis from the patient is not limited to KSA, "but this also happens in the West. And because if you're working in a multi-cultural, even in the UK, where you work, you have people from diverse, um, cultures, so it's similar as here". Gabir and Ahmed describe how they would approach the patient and ask if they know about the disease they have, and take the patients lead on how much to tell them, "I ask the patient, do you know anything about your disease? Is he interested to know anything about his disease? So if he's interested or asked me 'what is my disease?', I will tell" (Gabir). All three would also try to negotiate with the family, "sometimes 
some doctors impacts with the family, like saying to family that's wrong, and according to science you have to tell him, and so on, I believe this approaching is not good, because it will not take them to a positive results, but oppositely, the family will hold on more on their opinion, and they will lose trust in the doctor, my belief, is to stand -at first with the family view, and tell them the good they do about caring for the patient, and that is true, because they are refusing telling the patient his disease and state only as a care for their patient psychological state and as they try to make him happier. After that you have to start to tell the family about the scientific view, but also you have to tell them that the science was made according to the Western culture, and there is differences between culture, but then also tell them that according to our experience with our patients in this community, telling the patient about the disease and state will be positive for him after all. Most of the family got convinced by this approach, and then they thanks the doctor after telling the patient" (Chokri).

Gabir and Chokri admit that on occasion they will concede to keep the diagnosis from the patient, "But we must admit that there are some families refuse at all to tell the patient, and we respect their culture" (Chokri).

\subsubsection{Respecting culture}

Chokri, Gabir and Dakheel point out that in regards to patients, KSA is not very multi-cultural. The vast majority of patients will be Muslims, from KSA. For the participants who are from KSA, and for those from Arab Muslim countries, the culture is very familiar. For this reason, the Muslim participants tended to focus more on the relationships within a multicultural healthcare team, whereas the two 
participants from non-Muslim countries, Gail and Hailey, spoke of their interaction with the culture of KSA, via their patients.

Gabir explains "You know that in Saudi Arabia the situation is not like the Western countries where you deal with different and various cultures, here in the hospital with $90 \%$ we are dealing with one culture and it's the Saudi Muslim culture, so I didn't experience the cultural difference that much but we as a team we respect the different cultural and religious background, and we deal with them with the same importance and care, but to be honest we don't have multi cultures here in Saudi Arabia, more than 90\% it's just one culture Saudi Muslim but we as a team we can deal with cultural differences with all elastic and smooth."

However, some patients may find the presence of non-native health professionals problematic, "Saudi patient, the patient won't reacts the same way if the doctor was Saudi or Egyptian or Syrian or whatever and he reacts in a bad way if the doctor isn't Saudi. And he will hear and understand better if the doctor or anyone in front of him is Saudi. So sometimes we have to ask the patient relation administration for help to be a connection between the patients and us. But there is no much of this type of cases. But it happens" (Bandar).

The importance of respecting the culture of patients was recognised, "we react to the patient according to his culture, according to his cultural background" (Chokri), "every patient you must react with him according to his culture" (Ihsan). Both Gail and Hailey talk about the importance of respecting the culture of their patients, but at the same time finding it difficult to understand some of the beliefs, "it's hard for 
someone from my culture......to understand that belief but never the less it is a belief" (Hailey), "I cannot...because me, I am a professional, and then here some, some people are not educated sah (right)?...So it's a big difference. It's their culture already; they grow up with this culture" (Gail). Gail expresses a need to accept the presence of certain cultural beliefs, to be able to work with the patients, "But if I will go beyond their perception, they will not accept my suggestion...So I cannot just remove the culture, what they grow up in the culture. We cannot just tell them to forget about it. You learn from this thing" (Gail).

Specifically, participants mentioned the belief in the evil eye or sorcery as a cause or contributory factor in illness. This was very difficult for the participants who were from non-Muslim countries to understand, "I mean it amazes me even educated people to...they still have those umm ...the evil eye has ahh...affected my mother or whatever" (Hailey). Hailey felt that the belief in the evil eye had a negative effect on the patient's mental state, as it made them feel powerless to recover. The native participants were more likely to be accepting of this belief, and Ahmed mentioned that spiritual care had a role where these beliefs were present, "but their perception of diseases is quite different here...some people think it's, umm, the evil eye- the cause of cancer; Some probably, they perceive it, it's, uhh, because of, uhh, sorcery (Seher in Arabic)...some people think, they have a perception, ok. And that's why with spiritual care helps in that part. It might also, uhh, address these things as well" (Ahmed).

However, Emad showed less sympathy towards some of the alternative healthrelated beliefs of the patients, "the health education is bad, they believe in some 
kind of cures like the herbal treatments, they believe it will help and cure the patient, but in fact it won't, it's just additional cost and efforts, but because of the low education and ignorance they believe it will help".

Gail spoke about respecting the culture of her patients, yet when she speaks about the division of males and females in Saudi culture, she talks about breaking this custom in a way she believes is beneficial for the patient, "As much as possible we can talk to the patient, comforting the patient. That will be a relief to the patient, and sometimes they are touching. But here you know Saudis, we are not allowed to do. Touch therapy is very good for the patient, especially those dying patients...But we are not Saudi people, that's why we can do whatever we can do for the patient's good, for the patient's feel, for the improvement of the patient. Touch therapy is very nice" (Gail).

\subsubsection{Role of religion}

Similarly, the role of religion was more of an issue for the participants from nonMuslim countries, who had not grown up with these beliefs. They appeared to struggle with some aspects of the expressions of faith, "in some cases it causes a real umm sense of anguish because it's God's will that my family member died. I don't question God's will. On the other hand, why did he have to die he was only twen...twenty-eight years old, they're questions but I'm not supposed to question and I'm not supposed to grieve" (Hailey).

Gail describes her attempts as an outsider to understand the rituals performed for the patient near to death, "So the 'mutawa' will come and then as far as I observe 
aha, they will murmur any one prayer, like that, and then they will whisper to the ears of the patient. And then they will blow something, "bff, bff, bff, bff", like that on the face of the patient. And then they want maybe to remove the bad spirit. That is inside the patient. Remove all the bad spirit and then, then sometimes they are singing, but mostly they will say in Arabic about their prayer. That's all, that's what I observed from 'mutawa' in KSA' (Gail).

Despite not always being able to understand the religious rituals, and seeing some negative aspects, both Gail and Hailey also saw the positive impact of faith, "So spirituality is good because it gives strength, you have a belief or religious beliefs" ... when I go into a room, and the family is sitting reading the Holy Quran and they'er giving the ZamZam (16:14) and making sure the patient's [inaudible 16:18 - 16:20] I think that helps the patient and the family members"(Hailey).

Hailey also recognised that despite the majority of patients being Saudi Muslims, they also varied in their expression of religion, "So it depends on the individual, depends on the family, depends on even the region where they live perhaps".

Both Ihsan and Emad speak with an easier understanding of the religiosity of their patients, but also recognise that there can be both positive and negative expressions of faith, for example, "spiritual leader role is important, they will grow the hope in the patient, like when they tell him the disease is a forgiveness for sins and so on, they also tell him you cannot tell if he is going to die, no one will never know, so they can use their ways to provide hope for the patient...Here we are all Muslim, and fortunately we believe in the destiny, I consider it as something very 
beautiful, so any disease or accident is in the range of destiny, so when the patient and his relatives are wise and faithful you find him nice, and react in a very good way, on the other hand we also receive very bad cases, we find it hard to communicate with them" (Ihsan).

\subsection{Summary of culture}

It is clear that KSA presents unique challenges to the healthcare provider. The issue of truth telling appears to be in transition, with some participants having an open communication with patients about their illness, and others maintaining the traditional stance of protecting the patient from losing hope. The participants expressed the various ways in which they showed respect for the culture, and it is evident that this is a necessity for the practice of palliative care. Religion was seen as playing both a positive and negative role in the way the patient experienced what was happening to them.

\subsection{Future}

Those participants with a broad knowledge of palliative care were able to discuss plans and hopes for the future of palliative care. They outline their vision for the future, including some examples of current initiatives, and what they felt was needed.

\subsubsection{Vision}

Ahmed, Gabir and Chokri, palliative consultants at hospitals with current palliative care provision/education, were planning on further improvements, in both provision and education "We're looking further into, umm, improving it and having 
a program that trains people more in this, uhh, aspect. ...our vision is to have more palliative care physicians, nursing, and more centres specialized in palliative care" (Ahmed). Bandar hoped to see a separate department for palliative care within his small hospital.

Chokri specifically mentioned a proposal for a palliative care centre (mainly educational) and ultimately, a 'hospice service', and Emad and Dakheel both spoke about hopes for a palliative care centre, "I wish one day in the future I see a centre for palliative care with a qualified staff. Because there is a lot of patients who need palliative care, who for example patients with cancer and chronic illnesses, there are a lot of them!" (Emad). Hailey, who works at a hospital which already has a division of palliative care, mentions opening up palliative care for diseases other than cancer.

On a wider level, Ahmed and Gabir also look forward to changes at policy level and ultimately, to improvements to palliative care throughout KSA "First of all as I said, the presence of the organization Saudi Palliative care society to adapt all the common causes related to the palliative care and deliver recommendations for example to the $\mathrm{MOH}$, cause this is the needs of the palliative care, this is our view of the palliative care. It'll be a very positive and helpful, and with it we won't narrow the palliative care only in the main cities and hospitals but spreading it to all the hospitals and all the cities" (Ahmed).

Hailey gave a more detailed account of what she would like to see in terms of palliative care throughout KSA, "what I would recommend is that all major hospitals 
and urban areas whether their academic facilities, tertiary care facilities, have palliative care units or at least palliatic care teams with designated beds for care patients in need. I think it would be most effective and cost effective if the smaller communities had umm utilized their primary healthcare clinics who train people, physicians, nurses, perhaps social workers, to work out in these primary healthcare clinics as palliative care teams within these centres... but one thing I think would be really useful and not just for the national guard but extending this concept of taking palliative care out to the community. We know that there are many communities up in the mountains of Abha or out in the dessert somewhere out in the eastern region wherever. They don't even have access to primary healthcare clinics at times. I think, and it's been proved effective in countries in Africa, in India, and elsewhere, if you have mobile clinics, you know mobile vans".

While Hailey expresses frustration that there is so much that could be done and the money within the country to do it, but progress is slow, Chokri is optimistic about the future of palliative care in KSA, "So palliative care has a great future in KSA and there will be a great demand for it...it'll go from good to the better and the expansion in the palliative care".

\subsubsection{Needs}

Both Ahmed and Chokri mention the need to raise awareness of palliative care with KSA, "It's a new speciality. I think awareness. I think we just started our program. Hopefully, we can spark..spark out more physicians and train the physicians. Also, you have to promote your speciality. If people don't know about what's your speciality is, nobody is gonna come" (Ahmed). 
The idea of dissemination of palliative care from the larger hospitals with the current provision, to local hospitals and more rural areas, was mentioned by several participants, as a way cost effective way of spreading palliative care practice, "so our plan is to train more physicians. Maybe they can go outside of Riyadh; they can start their own programs, and this will spark up a collaboration and have more physicians being all over the kingdom inshallah (God willing). So this is our..our vision in palliative care" (Ahmed), "As I said before, I wish the ministry provide one or more of the palliative care specialists for any hospital, and this will spread the culture of palliative care around in the future" (Farah). Hailey emphasises the need for the spread of knowledge of basic principles of palliative care, "it needs to be out there in the community, in the primary healthcare facilities at a general level where they understand pain and symptom management, not in great depth but its...it's there, it's available, there is knowledge, and that's not happening" (Hailey).

While Chokri was optimistic about the future of palliative care in KSA, he also mentions limits to this development and does not see that the adequate amount of palliative care staff will be achieved in KSA in the near future. "I do not think in the future that we will reach to adequate minimum number of staff. No physicians, nor nurses social workers and others. I do not think!" (Chokri).

Education is seen as key to the development of palliative care, "Make the residency program for the palliative; the attached doctor should have a residency program in the addition of the fellowship to have his board and to develop himself during working in the palliative care" (Farah). Similar suggestions were made by Chokri and Bandar. 
Of all participants, Hailey's vision was the most creative, and this may be due to her being an 'outsider', and seeing things objectively, but also being highly personally invested in the country (as she spoke about herself as 'half-Saudi', "I would love to see, I would love us to have some open forums, I would love us to have some groups, I would love family members to be able to come in groups and sit and share with... whether it's [hesitation] television station or radio or whatever just to share...to share the burden but we're not there yet". (Hailey)

\subsection{Summary of future}

Education and training were central to the participant's vision of the future of palliative care, as well as increasing public awareness. Examples of future visions included having exclusive palliative care centres and expanding provision to remote communities. It was accepted that changes were needed at a policy level to effect real change.

\subsection{Conclusion}

This chapter has described the key themes and sub-themes drawn from the participant interview data. While many themes related specifically to the interview questions, other themes emerged from the data as areas of importance. The analysis of the data was descriptive, as it was felt that there was great value in allowing the voiced of these health professionals to be heard, and therefore my own interpretation as a researcher has been minimised.

The participants defined palliative care as concerning the patient and their family, for people with an incurable illness. They felt it concerned death, dying and the end of life, and necessitated a holistic view of the patient. Responses varied between participants, 
some being extremely well informed, and others having a limited view of what palliative care is, with one participant not having heard of the term prior to the interview. Participants saw palliative care as a unique or rare specialisation, having both positive and negative points, and as a specialisation which was growing in need and importance.

Doctors and nurses perceptions of palliative care were described by the participants, and it was evident that participants felt that palliative care was not well understood or seen as an appealing discipline. The importance of teamwork as identified, and significantly the importance of the personal qualities of the individual health professional in the standard of palliative care delivered.

Participants expressed a variety of issues and problems with palliative care, including communication, particularly language barriers between the health professionals and the patients, and a lack of resources, particularly not having sufficient adequately trained staff. Not only were resources insufficient, but there was thought to be poor allocation of existing resources.

The need for knowledge, training and education was acknowledged by all participants. A good level of palliative care knowledge was believed to exist within larger hospitals but not to have reached smaller and more rural hospitals. It was evident that KSA is in the process of change in this regard, and several educational initiatives were cited. The need to educate the general public on palliative care was also mentioned. This links to the role of media in influencing public perception of palliative care, which was seen as positive, negative or non-existent, depending upon the individual experience of the participant. 
Medication was discussed, specifically the issue of availability, and while availability itself did not seem to be a problem for most participants, the protocols and processes involved in administering medication may be disadvantageous to the patients. The protocols were seen as important and had developed from the misuse of medication in some instances. It was also suggested that some health professionals and patients might have reservations about medication stemming from cultural beliefs.

Participants varied in their knowledge about legislation on palliative care. Most but not all were aware of their own hospital policies, and gaps in policy were noted. Policies and protocols were seen as important in delivering consistently good palliative care.

While not specifically asked about, culture emerged as a theme, as delivering palliative care in KSA has its own unique cultural issues. 'Truth-telling' of the diagnosis to the patient was a significant issue with participants expressing varying stances in relation to this issue. This is linked to the sub-theme of respected culture, which most participants acknowledged was necessary in order to provide good palliative care, but this also presented challenges. Also linked to culture was the role of religion, which has implications for the way palliative care could be delivered and was seen as having both a positive and negative impact on the patient and family's response to illness. Finally, participants expressed their vision of the future of palliative care, including current initiatives, and also the needs of palliative care which were largely centred on education and training.

The discussion chapter will evaluate the findings, in the light of the methods used, and in comparison to extant literature. This will help to ascertain the value of the present research, and how it contributes to the wider research landscape. 


\section{Discussion}

\subsection{Introduction}

The aims of this study were to 1) investigate and describe the perceptions of the KSA hospital nurses, physicians, and healthcare administrators regarding palliative care; 2) identify issues that KSA hospital nurses, physicians, and healthcare administrators have regarding palliative care; and 3) develop recommendations for strengthening the value of palliative care among healthcare practitioners in KSA. To this extent, semi-structured qualitative interviews were undertaken with ten currently practising healthcare providers in KSA.

This chapter begins with a summary of the key findings, followed by a discussion of these findings within the wider context by comparing with research presented in the literature review. This will lead to a discussion of the contribution of the present research to the wider research environment and finally the implications of the research, for palliative care in KSA.

\subsection{Summary of findings}

Palliative care is now a well-defined concept within the developed world and an accepted specialism within many countries. However, in KSA, as elsewhere in the Middle East, palliative care services are as yet patchy and localised.

The contribution of the present study to the understanding of the perceptions of healthcare workers in KSA towards palliative care is significant considering the scarcity of research in this area, particularly qualitative research. It supports the findings of previous research into palliative care in KSA and the wider research on 
palliative care. It also provides a unique insight into the views of healthcare workers, expanding upon current literature.

The findings suggest that while some participants define palliative care in a way which is in keeping with internationally recognised definitions, others had not heard of the term, or defined it in a way which was incomplete. Insights were provided into doctors and nurses experiences of palliative care, an example of which is that the personality of the healthcare provided was a significant factor in the quality of palliative care provided. The findings suggested that as a specialism, palliative care is seen as unique and growing, and participants understood the need and importance of this area. Issues and problems with palliative care were identified, including issues with communication and lack of material and human resources. Various opinions were offered as to the role of the media in promoting palliative care in KSA, issues with medication, including access and procedures, were discussed, and finally the participants spoke about their vision of the future of palliative care in KSA, and what would be needed to achieve this vision.

\subsection{Comparison of findings to literature review}

\subsubsection{Defining Palliative Care}

The interviews highlighted healthcare providers' understandings of palliative care in KSA. It did so by asking participants to give their understanding of palliative care which then enabled a comparison between the participant's perception and the current definitions of palliative care. The participants' understanding of palliative care tended to agree with the definition of the speciality present in Western literature on the topic, although participants varied as to their awareness of this. 
WHO define palliative care as "an approach that improves the quality of life of patients and their families facing the problem associated with life-threatening illness, through the prevention and relief of suffering by means of early identification and impeccable assessment and treatment of pain and other problems, physical, psychosocial and spiritual"(World Health Organisation, 2015).

When participants gave their understandings of palliative care, they overwhelmingly emphasized the importance of the family, which is also an accepted element in palliative care within the literature (Amoah, 2011; World Health Organisation, 2015). This is a valuable finding, as the focus on the family has important implications for providing palliative care in KSA. Gatrad \& Sheikh (2002) note the norm of the extended family structure within Muslim countries including KSA. As this PhD research study illustrates, the family proved important in providing palliative care for patients suffering incurable illnesses in KSA and in particular, families played a major role in the communication of the patient's illness diagnosis and health status.

Also, their definitions reference incurable illnesses as well as death, dying and end of life. This is in keeping with the traditional view of palliative care. However, countries with a long history of palliative care, such as the UK, are moving towards a model which introduces palliative care earlier in the illness trajectory, alongside curative treatments, as this has been shown to be the most effective approach (Mir, 2011). It is not clear whether KSA is following this model at present, but a study by Gray et al. (1995) found that healthcare providers in KSA tended to focus solely on 
curative treatment plans for cancer patients, even when palliative care should already be considered as the best option.

The participants also see palliative care as relating to cancer patients, and this is a reflection of the organisation of palliative care within KSA, for example, KFSH\&RC considers palliative care as a branch of oncology. This is in contradiction to the Western model of palliative care, which has evolved over time to define palliative care as something which may become relevant much earlier in the course of the illness, and even prior to death being certain (Mir, 2011). Palliative care in the west, while also being closely linked to cancer care, is seen as relevant to all illnesses and diseases which may result in death (WHO, 2015).

Finally, their definitions note the holistic view of palliative care in that it not only involves providing physical care of a patient but social, spiritual and psychological care as well. For example, Chokri refers to the impact of beliefs on behaviour. This is in keeping with the concept of 'total pain', coined by Cicely Saunders (Clark, 2004), and holistic care as defined by NICE (NICE, 2004), which should integrate the physical, social, psychological, occupational and spiritual needs of the patient. However, there were many examples given of how this is difficult to achieve in KSA, partly due to the majority of patients being Muslim, and being treated by nonMuslim health professionals who may find it difficult to understand their beliefs and incorporate them into the care giving relationship.

The accuracy of the participant's definitions varied by the level of palliative care provision in the particular hospital they worked in. For example, in KMAC, nurses 
and physicians are not experienced and trained in palliative care. Farah's

understanding of palliative care did not match a holistic definition of palliative care, such as, for example, the definition that is given by WHO. She said "As I said before, to take care of the patient, so he becomes pain-free, just to remove the pain and the symptoms resulted by the disease, regardless what is the disease itself. All

symptoms." The perception of palliative care is that it is used when the end is near and after chemotherapy. This is contrary to the current understanding of palliative care in the West, which sees palliative care as being provided at an earlier stage in the disease trajectory, alongside curative treatments (Mir, 2011). Farah only sees the palliative care as managing pain and treating symptoms. She did not appear to understand that palliative care has more psychosocial and spiritual elements that also need to be taken into consideration. Farah assumes that spiritual care is only given when there is no cure for the disease, whereas in fact, spiritual care should be present throughout the disease trajectory. However, Farah's definition did acknowledge the importance of pain management in palliative care.

On the other hand, Hailey had a clear understanding of the definition of palliative care, awareness of the WHO definition, and she was also aware of the extent to which palliative care was not understood within KSA.

An important element of palliative care covered extensively in the literature is the quality of life, which is seen as a central aim of palliative care in the West and is emphasised by WHO (2015). Those participants with more experience of palliative care mentioned the quality of life in their definitions, whereas others did not, which may be due to the association of palliative care with imminent death. 
In addition to the above, it also appears that the concept of advanced care planning is not known or practised in KSA.

\subsubsection{Perceptions of PC as specialism}

The first sub-theme within this theme was the perception of palliative care as a unique or rare specialism, and the second sub-theme was that it is a growing specialism, in both KSA and the world. This reflects the current literature, which sees palliative care as an emerging specialism in middle eastern countries (Webster et al., 2007), while in the UK it is already established as a sub-speciality of medicine, with its own training and exit exams (Fallon and Foley, 2012). With an ageing world population, palliative care can be seen as a growing discipline worldwide (Wright et al., 2008), indicating that the participants had an accurate perception of the place of palliative care in KSA and the world.

Participants highlighted the value of palliative care but emphasised the lack of trained professionals in palliative care in KSA. This is well documented in the literature (Ghanem et al., 2011; Zeinah et al., 2013; Abudari et al., 2014), and again indicates the accuracy of the participant's perceptions.

\subsubsection{Doctors and Nurses}

The participants interviewed believed that doctors and nurses did not have a good understanding of palliative care. This is supported by evidence that referrals to palliative care in KSA happen late in the disease trajectory (Gray et al., 1995; AlShahri et al., 2010), and that good knowledge of palliative care results in earlier referrals (Al-Shahri et al., 2010). The participants also noted that to attract doctors and nurses towards palliative care as a specialism, understanding of palliative care 
needs to be increased. This has also been noted before in the literature. Almuzaini et al. (1998), found that two-thirds of Saudi physicians showed a negative attitude towards working in palliative care, compared to $90 \%$ of non-Saudis who showed a positive attitude.

The participants recognised the importance of palliative care being delivered by a team of professionals, given its holistic nature. This is something which has become the norm in the UK and other developed countries (Fallon and Foley 2012) and indicates an accuracy of understanding by some participants of an appropriate model for palliative care.

An interesting finding was that participants recognised the importance of the personal characteristics of doctors and nurses in delivering good palliative care. This was not explicitly mentioned in the literature reviewed, and possibly indicates that in countries where palliative care is well established (which is where most of the literature comes from) effective training and efficient systems may make palliative care less dependent on personal qualities. The literature did refer to the importance of understanding the patient's culture (Nyatanga, 2002; Mir, 2011), and mentioned the impact of the individual on delivering the spiritual aspect of palliative care, for example, aspects of communications such as active listening and validating emotions (Amoah, 2011).

\subsubsection{Issues and problems}

Communication was raised specifically by participants as an issue, both in general and specifically with regards to language, noting that the majority of nursing staff to 
do not speak Arabic, which is the most likely language of the patient. This is in agreement with the findings of other KSA based studies. A study by Alshaikh \& Sormunen (2012), which found that nurses faced challenges in communicating with patients and their families due to language barriers. A study by AlYateem \& AlYateem (2014), suggested that nurses felt compromised in giving adequate care by their lack of ability to speak the patient's language. A lack of communication may heighten the patient's distress (Amoah, 2011). Nyatanga (2002) noted that language barriers were particularly difficult to overcome within palliative care, and suggested the use of formal and informal interpreters.

Human resources were unanimously agreed by participants to be insufficient to provide good quality palliative care. Unsurprisingly, this reflects what is found in research literature into palliative care in KSA, which, despite developments, remains inadequate and leads to patient suffering and high death rates in hospitals (Almobarak 2014). Without adequately trained staff, those elements of palliative care which tend to indicate quality can be compromised. Early referrals to palliative care were compromised by lack of confidence, knowledge and skills of healthcare providers (Gray et al., 1995a; Al-Shahri et al., 2010; Alsirafy et al., 2010). Time between last chemotherapy and death (TLCD) can also be compromised by a lack of skill in palliative care and was found in KSA to be shorter than the world average, therefore indicating aggressive end of life care (Karim et al., 2015). Similarly, AlShaqi et al. (2012) found that the use of antimicrobials was too liberal in patients approaching death, again indicating aggressiveness of end of life care. 
Gabir specifically mentioned that there were no nurses specialised in palliative care in his hospital. The literature supports that there is a lack of specialised carers and further, suggests that not only are nurses not specialising in palliative care, but they have inadequate knowledge of it, and their training in it is insufficient (Jazieh et al., 2010; Abudari et al., 2014; Alshaikh and Sormunen, 2015; Youssef et al., 2015).

Abudari et al., (2014) suggest that due to the varied background of nurses in KSA, leading to a lack of homogeneity in palliative care skills, palliative care should be an integral part of orientation training to work in KSA. The findings of the present study, which highlight discrepancies between healthcare workers in regards to knowledge and understanding of palliative care, indicate the appropriateness of this suggestion.

One participant noted the lack of any sort of spiritual care specialist within their hospital. Spirituality within palliative care is seen as being equal to physical and other domains in the experience of dying for patients and families (Puchalski, 2012), and therefore this matter is of great importance. It is not clear in the present study who should provide the spiritual care, for example Chokri discusses a spiritual care specialist but does not state the background and qualifications of that person. However, Gail seemed to see an element of spiritual care being woven into her nursing practice, which is more in keeping with the literature which sees spiritual care as being provided primarily by nurses, but also by social workers and psychologists (Kellehear, 2000). However, the situation in KSA is different from the UK, due to the dominant religion being Islam. Kellehear (2000) notes that modern palliative care discourse has distanced itself from the discussion of God and 
religion, preferring the more neutral territory of spirituality. However, those involved with patients at the end of life in KSA would need some knowledge and sensitivity to religious needs.

Finally, Hailey notes that despite ample wealth within KSA, palliative care does not receive adequate funding. If this is indeed a fact, this may go some way to explaining the lack of progress that can be seen from reviewing the literature on palliative care in KSA over the last twenty-five years. Since the opening of the first palliative care facility in 1992, there has been no national action to improve palliative care, and aside from what is provided in the primary health-care system, it remains a matter of local provision (Abudari et al., 2013).

\subsubsection{Knowledge, training and education}

The interviews revealed serious gaps in the knowledge of healthcare workers in KSA in relation to palliative care. For example, Farah, although a palliative care physician, sees palliative care as only applying to cancer patients, whereas current literature states it can be given to a patient with any incurable illness. She also does not know if there are legislation and constitution regarding palliative care or not. This participant suggested that most practitioners well educated in palliative care could be found in the larger hospitals, and identified a need for palliative care to be part of the undergraduate curriculum, as well as 'on the job' training.

Participants noted a particular need for training of nurses, as while there were nurses in KSA hospitals who were considered 'palliative nurses', this was due to their working within the palliative care team, not due to any training in the subject. 
This is reflected in the findings of studies by Youssef et al. (2015) and Alshaikh \& Sormunen (2012) suggesting that more than half of the nurses were found to have poor knowledge of palliative care. At the same time, it reflected the challenge identified by Al-Awamer and Downar, (2014) on the challenges faced by palliative care in Middle Eastern settings. As discussed by Al-Awamer and Downar, (2014), healthcare professionals' unfamiliarity with their role in the palliative care of patients is a significant problem in hospitals in the Middle East and this is mainly due to the lack of training and development opportunities for them in this area, Abudari et al. (2014) found that there was a large variance in palliative care education of nurses depending on the country of origin. The emphasis on nursing care by participants is in keeping with the view that good nursing care is a central element of quality palliative care provision (Abudari et al., 2014). Moreover, the literature suggests the effectiveness of educational interventions for nurses in improving palliative care (Abudari et al., 2014). Thus, findings from this study coincide accurately with what is known in the literature to be happening in other countries in the Middle East. This shows that the problems facing palliative care in the KSA are not unique to it, but is shared by other countries with the same sociocultural background.

\subsubsection{Educating others}

Participants also mentioned the need for educating others, meaning the general public, in regards to palliative care. It was seen as particularly important to educate the family on what palliative care is, as well as teaching the family how to care for the patients. The 'low health culture' was also mentioned, such as the belief in 
herbal treatments and other interventions that may be contrary to modern medicine. The literature mentioned the fear of opiates as a particular barrier to providing effective palliative care (Al-Shahri et al., 2012). Regarding more widespread education, KSA may benefit from implementation of the WHO public health strategy (Stjernswärd et al., 2007)

\subsubsection{Role of media}

Due to the lack of literature about the role of media in palliative care in KSA, participants were specifically asked what their views were about this. The responses were varied. Two participants felt the media has a positive role, for example, they had been in attendance at a palliative care awareness day in the participant's hospital. Five felt they had no role, that is, they had seen no evidence of it, and three felt they had a negative role, for example, spreading rumours on social media. The WHO (2015) state that engagement with the media is an important element of palliative care education, suggesting that it is important that those involved in providing palliative care in KSA engage with the media in a positive way.

\subsubsection{Medication}

Most participants indicated that availability of medication was sufficient within their hospitals. However, Emad alone noted that there was insufficient at his hospital. Availability, therefore, may vary between hospitals, and where the patient lives in relation to the hospital. Pain relief is central to the definition of palliative care (WHO, 2015). Without effective pain control, other goals of palliative care may 
not be achieved, and physical symptoms have an impact on psychological symptoms such as stress and anxiety, (Vachon et al., 1995).

A study by Al-zahrani, Eldali, \& Al-Shahri (2014) found that $85.5 \%$ of cancer patients in KSA reported severe pain, compared to $65 \%$ in an international review. This suggests that despite the apparent availability of medication, it is not always being prescribed, administered or used effectively.

As found by Al-Shahri (2009) and Alshammary et al. (2014), the per capita consumption of morphine in the country is less than $0.35 \mathrm{mg}$, which, when compared to the worldwide consumption of $5.6 \mathrm{mg}$ shows that people in KSA are using much less pain medication than people in other countries.

In the descriptions given by the participants as to the drug procedures at the various hospitals, it is clear that there are a number of administrative processes that must be conducted before morphine can be given. While these were described as necessary by the participants, to avoid misuse, it may be that these procedures result in delays in effective pain relief for the patient, e.g., "it must be given after doctors' signature, and there must be a person with the nurse to check that the nurse did the injection in the right way" (Ihsan). Bandar explains how it was misuse of medication which led stricter procedures for administering medication in his hospital. It is acknowledged within the literature that concerns about misuse of opioids, and consequently their availability, is a problem in many non-Western countries (Webster et al., 2007; Aljawi and Harford, 2012).

Farah admitted that in the past, she did not give attention to the patient's disease, but she was more worried about side effects of the medication "we focused on the 
side effects more than the disease itself". In this regard, as discussed prior in this thesis, opioids are considered acceptable in Islam when necessary (Al-Shahri \& AlKhenaizan, 2005), particularly where the relief of pain for the Muslim patient may enable them to engage in spiritual acts that would otherwise be impossible (Choong, 2015). However, Choong (2015) suggests that patients may have reservations about using such drugs. This was not mentioned specifically by the participants, although, Gail described how the patient and their family might wish to use traditional medicines which have no evidence of being effective, and this may impact upon the use of modern medication.

Chokri mentions the importance of educating other departments, particularly the pharmaceutical department, on the use of medication within palliative care. Farah also describes her own experiences in administering medication. She was fearful of the patient becoming addicted to pain medication, which has been identified in the literature as an aspect of culture in Middle Eastern culture, "We were not so generous with the patient about the pain killers because we were afraid he would be addicted to the painkiller".

This is supported by the findings of Alshammary et al., (2014), who acknowledges that the lack of training and confidence of healthcare staff in using medication results in inadequate pain management.

\subsubsection{Legislation and Policy}

Participants varied in regards to their knowledge on legislation and policy regarding palliative care. Some, such as Gabir, advocated a bottom-up approach to policy, 
educating the people delivering palliative care before trying to make changes at government level. Others, such as Chokri, believed changes at policy level would make a difference.

The World Health Organisation have been promoting pain relief and palliative care since the early 1980's (Wright et al., 2008), and recommend that each country has a national policy for implementing palliative care (Webster et al., 2007), suggesting that adequate policy planning is seen as an important foundation of palliative care. Participants emphasised the need for good policies and protocols within hospitals, which, they stated, would unify practice and spread good palliative care throughout all facilities, from the larger to the smaller hospitals. This view is supported by studies into aspects of palliative care, such as referral times and aggressiveness of end of life care in Saudi Arabia, which indicate that these aspects (e.g. Alshammary et al., 2014; Karim et al., 2015) could be positively affected by policies which could be implemented as protocols within hospitals.

In the UK, since becoming established as a speciality in 1987, palliative care has become well established with networks of day centres and hospices serving palliative care needs. This can be seen as based on the pioneering work of Cicely Saunders, education initiatives and policy. KSA's' first palliative care provision was a two-bed facility at King Faisal Specialist Hospital, and Research Centre (KFSH\&RC)opened in 1992, but despite other isolated developments in local provision, there has been no establishment of a national palliative care initiative (Abudari et al., 2013). The literature and the researchers own experience confirms 
that a national palliative care programme in KSA (AI-Shahri et al., 2004) has not been adopted by the Saudi authorities.

An interesting finding of the study, which was not addressed within the reviewed literature, was the way that health professionals would work around policies which were inadequate or overly restrictive, to give the patient the care they felt was needed.

\subsubsection{Culture}

It was clear from the interviews that the participants saw care for the family as an integral part of palliative care. This is congruent with Western definitions of palliative care (Choong, 2015), and this element of Saudi culture may be beneficial to the development of palliative care in KSA. Culture in KSA is defined by an extended family structure, and care of the dying is an essential part of family life (Gatrad and Sheikh, 2002). In the work of Al-Awamer and Downar, (2014), it was also established that cultural barriers prevented palliative care from developing effectively in Middle Eastern settings. Families do not always understand the importance of palliative care for their loved ones and so may not always make the best decisions in relation to their care, yet hold considerable power over those decisions (Al-Awamer and Downar, 2014). However, Al-Awamer and Downar, (2014) found that family members were willing to learn and understand the value of palliative care with proper education, and this is something that was also echoed by participants in this study as a possible means for bridging the cultural barrier. Thus, for the successful development of palliative care in KSA, the involvement of the family needs to be perceived as a positive resource and harnessed as such. This 
would mean that palliative care in KSA would develop in a unique way, which would be appropriate to the KSA culture and therefore more likely to be successful.

Participants particularly emphasised the need for spiritual care for the family members. Tayeb (2010) recognises that while Islam helps family members to cope, they still have unmet needs. To ensure that the cultural differences between patients and families do not lead to inadequacies in the delivery of palliative care, the spiritual needs of families may need to be evaluated, in order to suggest potential ways to fulfil this need.

The literature suggests that it is normal within Saudi culture for the patients family to contribute to the decision-making process within palliative care (Al-Shahri and Al-Khenaizan, 2005), and this brings us to the issue of what has been labelled 'truth telling'. Truth telling is identified in the literature as an issue in relation to palliative care, which was created and developed in the West according to Western values and may transfer to other cultures. Similar to that found in other studies in KSA, and countries where there is a similar culture, the findings of this study show that physicians will not always grant the patient their right of knowing their diagnosis. Serious illness is supposed to be diagnosed by the Dr, such as oncologists, however, many patients are referred to palliative care without knowing their actual diagnosis. Once the palliative care providers receive the patient, they will give care until death. However, in KSA, if the patient is not aware of their illness, the physicians won't tell them the diagnosis. Chokri, despite his seniority as a healthcare practitioner, admitted to continuing this practice. 
This research study found that the family played an important role in whether or not a patient is aware of their diagnosis. Many patients were, in fact, not aware of their diagnosis. While in some cases a patient's mental capacity was not sufficient to be able to understand their own illness diagnosis, in other cases, family members feared that becoming aware of their illness diagnosis could negatively affect the patient's physical and mental health. As research participants indicate, while many felt it was the patient's right to know his or her illness diagnosis, due to the important role of the family, in many cases communication of this diagnosis to the patient becomes a negotiated process between the healthcare practitioner and the patient's family. This raises interesting issues regarding patient confidentiality. Firth (2001, cited in Gatrad \& Sheikh, 2002) observed that various non-Europeans, including Muslims, preferred non-disclosure to avoid loss of hope. However, a study by (Al-Amri, 2010) found that $98 \%$ of Saudi cancer patients wanted to know about their diagnosis and prognosis.

While in the West it may be hard to conceive of the fact that a patient's family may have more information about the patient's illness diagnosis than the patient, in less individualistic cultural environments where the family is of prime importance, such as is the case in the KSA, this is not surprising. One of the main issues healthcare providers in this study indicate, with regards to providing palliative care, involves communicating a patient's health status with members of the patient's family. As research participants in this study indicate, in many cases each of the patient's family members separately desired to speak to the healthcare provider regarding the health of the patient. This consumes a significant portion of the practitioner's 
time and presents a challenge for Saudi healthcare providers. It was noted by (Gray et al., 1995), that the norm is to view the patient as part of a larger family, who are responsible for the patient, provide consent for their treatment, and who consider it their duty to protect the patient from harm, which may include denying them information about their condition. This phenomenon is well documented in the literature, as occurring in Islamic societies (Gatrad and Sheikh, 2002). However, a more recent study by Al-Amri (2010) suggests that while this is still common practice, patient attitudes towards having information about their diagnosis may be changing, with $98 \%$ of patients interviewed saying they wished to know their diagnosis.

\subsubsection{Future}

All of the participants were keen to see further improvements in palliative care in $\mathrm{KSA}$, including the establishment of a palliative care centre, reaching out to noncancer patients and providing services to isolated rural populations. These suggestions indicate that the participants have a realistic perception of the current situation with KSA, for example, Ghanem et al., (2011) reported on non-cancer referrals to a palliative care service within in tertiary hospital in the Eastern Province of KSA, and found that $4.2 \%$ of palliative care patients had a non-cancer diagnosis. These were largely referred due to pain. This study also suggests the need for palliative care to reach more non-cancer patients.

Improvements were seen as occurring through training and education, and changes at policy level. This is reflected in the literature, with several studies that evaluate the quality of palliative care and attitudes to palliative care recommending that 
education and training of health professionals were essential (Alshammary et al., 2014), while others are suggesting a dual approach involving education and policy (Youssef et al., 2015). Other studies also show the effectiveness of educational interventions in improving palliative care (Abudari et al., 2014). These studies, alongside the results of this study, establish the link between proper training and development of palliative care in the KSA. At present, there remains a gap between what doctors and nurses know about palliative care and its relevance to their profession at the start of their careers, and what they know about it after spending a few years in their respective professions. This gap reflects a weakness of palliative care in the KSA that is borne out of its culture not considering palliative care as a priority in healthcare. It is this mindset that needs to change that the change needs to start happening in the education of the KSA's future healthcare professionals.

Some of the participants, such as Chokri, were optimistic about the development of palliative care, "so palliative care has a great future in KSA", while others, such as Hailey, were pessimistic, citing the availability of money and resources, but the lack of current progress. This lack of progress is reflected in the literature, with many of the same issues being found by studies throughout the last twenty-five years. On the other hand, Al-Shahri et al. (2004) have cited the palliative care provision at KFSH\&RC as advanced compared to other countries within the Eastern Mediterranean Region (EMR), and the potential to build on this may be a source of optimism. This shows the next set of improvements that should be strived for in developing palliative care in the KSA. Alongside improving the education of future healthcare professionals about palliative care, opportunities must arise for 
healthcare professionals to pursue specializations in palliative care in the country. There must be a movement within the industry towards recognizing palliative care as an important focus of discipline in healthcare, with the accompanying allocation of appropriate resources.

\subsection{Summary}

The contribution of the present study to the understanding of the perceptions of healthcare workers in KSA towards palliative care is significant considering the scarcity of research in this area, particularly qualitative research. While there were studies that have explored the development of palliative care in the Middle East, there has yet to be an in depth analysis of issues from the perspective of local healthcare providers in the KSA before this study. While some of the findings echo those of studies in similar contexts, others, particularly the findings on language barriers and palliative care as a specialization, retain some novelty. In general, the study supports the findings of previous research into palliative care in KSA and the wider research on palliative care. It also provides a unique insight into the views of healthcare workers, an expands upon current literature. The findings suggest that while some participants define palliative care in a way which is in keeping with internationally recognised definitions, others had not heard of the term, or defined it in a way which was incomplete. Insights were provided into doctors and nurses experiences of palliative care, an example of which is that the personality of the healthcare provided was a significant factor in the quality of palliative care provided. The findings in this $\mathrm{PhD}$ suggest that as a specialism, palliative care is seen as unique and growing, and participants understood the need and importance 
of this area. Issues and problems with palliative care were identified, including issues with communication and lack of material and human resources. Various opinions were offered as to the role of the media in promoting palliative care in KSA, issues with medication, including access and procedures, were discussed, and finally the participants spoke about their vision of the future of palliative care in KSA, and what would be needed to achieve this vision.

The following points summarise the unique findings of this research:

The acceptance of healthcare workers in KSA of the family as forming part of the definition of palliative care, and incorporating this into practice.

The importance of the personal characteristics of the individual healthcare worker in providing effective palliative care, including working around inadequate and restrictive policies to ensure the best possible care for the patient.

The need for spiritual care for the patient, which need to be provided within the specific context of KSA as a conservative Muslim country. 


\subsection{Implications and recommendations}

Based on the results of this PhD, two areas were identified for which there is significant implication. These are: for the future of palliative care in KSA, and for research. These are discussed further below.

\subsubsection{For the future of palliative care in $K S A$}

- The need for education in palliative care for all healthcare professionals, but especially nurses, was a strong theme. This is particularly important not only for native professionals but also those from overseas, as the varied backgrounds of the workforce accounts for a wide disparity in the understanding of palliative care.

- In a country such as KSA, where palliative care policy is lacking, good palliative care is often dependant in the personalities of key members of staff. Such staff may work around inadequate policies in order to provide the best possible care. Health professionals providing good palliative care should be encouraged to become leaders in the field, educating others in good practice.

- The emphasis on the family in KSA culture should be seen as a positive resource in helping to establish good palliative care.

- Policy, systems and resources need to be in place to support the development of palliative care throughout KSA, especially to disseminate good practice from the larger to smaller hospitals and rural areas. 
- Training needs to be provided to key members of palliative care staff to aid positive engagement with the media to increase public awareness of palliative care.

\subsubsection{For research}

- Researchers working in KSA need a good understanding of the culture and other factors which impact on the potential for research.

- Training should be provided in research skills, including ethical matters, to health professionals in Saudi Arabia, particular to native Saudis, as they will be in the best position to conduct research within their own culture.

- Due to the scarcity of qualitative research in this area, further qualitative studies would help to illuminate the issues.

- The areas of the family's role in palliative care, and of spirituality in palliative care in KSA are areas where research in scarce and further research would be beneficial.

\subsection{Conclusion}

Saudi Arabia faces many challenges in providing healthcare to its people, including a lack of skills in the native population, and the difficulties of providing adequate healthcare beyond the major cities. Palliative care exists in the major hospitals, but the development of these services has been slow and has not yet spread to the smaller hospitals or more rural areas. This study aimed to identify issues regarding the development of palliative care in the Saudi Arabia hospital setting, from the perceptions of healthcare professionals working in Saudi hospitals. These aims were addressed within a qualitative paradigm, drawing on symbolic interactionism and 
elements of grounded theory. The findings came from ten interviews within healthcare professionals working with terminally ill patients within KSA hospitals.

The findings chapter described the key themes and sub-themes within the data. The participants understood palliative care as care for patients with incurable illnesses and their families. They felt it concerned death, dying and the end of life, and necessitated a holistic view of the patient. The participants felt that palliative care was not well understood among doctors and nurses and that there was an emphasis on teamwork and the personal qualities of the health professionals involved in the quality of care delivered. Participants expressed a variety of issues and problems with palliative care, including communication, particularly language barriers, lack of resources, particularly not having sufficient adequately trained staff and poor allocation of existing resources.

The need for knowledge, training and education was acknowledged by all participants. The need to educate the general public on palliative care was also mentioned, and the role of the media in this was discussed. Issues around medication included availability, protocols and processes involved in administering medication, misuse of medication in some instances and reservations about medication stemming from cultural beliefs. Participants varied in their knowledge about legislation on palliative care. Most but not all were aware of their own hospital policies, and gaps in policy were noted. Policies and protocols were seen as important in delivering consistently good palliative care.

Culture emerged as a theme, as delivering palliative care in KSA has its own unique 
cultural issues. This included 'truth-telling' of the diagnosis to the patient, respecting the culture, which most participants acknowledged was necessary to provide good palliative care, and the role of religion, which has implications for the way palliative care is delivered. Religion was seen as having both a positive and negative impact on the patient and family's response to illness. Finally, participants expressed their vision of the future of palliative care, including current initiatives, and also the needs of palliative care which were largely centred on education and training.

This chapter has discussed the findings in relation to the literature review and those articulated by the participants. These findings provide a unique and difficult to obtain account of the views of healthcare professionals in KSA, presenting rich information which corroborates and elaborates on previous studies on palliative care in KSA. The present study provides an insight into the views of healthcare workers on palliative care in KSA, significantly adding to this topic area, substantiating previous research aiming to elucidate the issues and also identifying issues that have not been previously covered by research into palliative care in KSA. Issues not previously explored in the existing literature included the acceptance of healthcare workers in KSA of the family as forming part of the definition of palliative care and incorporating this into practice. Also unique was the finding regarding the importance of the personal characteristics of the individual healthcare worker in providing effective palliative care, including working around inadequate and restrictive policies to ensure the best possible care for the patient. Finally, the need for spiritual services for the patient was raised by participants, 
which needs to be provided within the specific context of KSA as a conservative Muslim country.

\subsection{Recommendations from the study}

One of the aims of this research was to make recommendations for the future development of palliative care in KSA. These recommendations are made based on the findings and also based on viewing the findings within the context of existing literature, as detailed in the discussion. Recommendations for clinical practice are that:

1. healthcare professionals providing good palliative care should be encouraged to become leaders in the field, educating others in good practice,

2. the emphasis on family in KSA culture should be seen as a positive resource in helping to establish good palliative care and maximised by staff to support optimum care,

3. policy, systems and resources need to be in place to support the development of palliative care throughout KSA, especially to disseminate good practice from the larger to smaller hospitals and rural areas, and

4. training is needed to assist key members of palliative care staff, to positively engage with the media in order to increase public awareness of palliative care.

In addition, this study makes several recommends broadly for KSA. These are that: 
1. training should be provided in research skills, including ethical matters, to all health professionals in Saudi Arabia, particular to native Saudis, who are better placed to conduct research in KSA than researchers from other cultures,

2. further studies should be conducted into the reason for the dearth of qualitative researchers in KSA, and

3. further research into the area of the family's role in palliative care, and of spirituality in palliative care in KSA are needed.

This PhD researcher acknowledges that undertaking research in KSA is challenging and researchers working in KSA need a good understanding of the culture and other factors which impact on the potential for research. Regardless of these issues, this research provides insight into palliative care in KSA, by exploring the views of healthcare professionals, and was motivated by the researchers own frustrations at the inadequacies of palliative care within KSA. It is not easy to obtain such views in KSA, where much is hidden, and people fear that speaking openly may cost them their jobs. This makes the findings even more valuable. It is the researcher's sincere hope that they will provide a positive and unique contribution to research on palliative care in KSA and ultimately have a positive impact on the development of palliative care in KSA. 


\section{Conclusion}

\subsection{Introduction}

The aims of this study were to 1) investigate and uncover the perceptions of the KSA hospital nurses, physicians, and healthcare administrators regarding palliative care; 2) identify issues that KSA hospital nurses, physicians, and healthcare administrators have regarding palliative care; and 3) develop recommendations for strengthening the value of palliative care among healthcare practitioners in KSA. To this extent, semi-structured qualitative interviews were undertaken with ten currently practicing healthcare providers in KSA.

This chapter will discuss the strengths and weaknesses of the study, reflect on the $\mathrm{PhD}$ journey and conclude the thesis

\subsection{Strengths of the study}

The findings from this research study contribute to the wider research environment in the following ways:

- Providing a unique and difficult to obtain account of the views of healthcare professionals in KSA,

- Presenting rich information which corroborates and elaborates on previous studies on palliative care in KSA,

- Providing an insight into the views of healthcare workers on palliative care in KSA, significantly adding to this area, and substantiating previous research aiming to elucidate the issues and 
- Identifying issues that have not previously been uncovered by research into palliative care in KSA.

\subsection{Weaknesses of the study}

\section{- Evaluation of methodology and methods}

This study was based on the idea that knowledge is subjective and therefore the best way to understand the phenomena of palliative care in KSA was to obtain the views of the people engaging with it on a day to day basis. Furthermore, a qualitative approach allowed for the findings to emerge from the data, and lead to unique findings in a way which a quantitative approach would not have.

\section{- $\quad$ The semi-structured interviews}

Smith et al. (2009) describe qualitative research interviews as a "conversation, with a purpose" (p57), the purpose of which is informed by the research questions. The use of semi-structured interviews allowed enough control over the conversation to ensure topics of pre-identified importance were covered while allowing enough scope for participants to express their personal views and introduce new topic areas. As such, the method used was effective, and the findings are a combination of themes based on the original broadly structured questions, and new themes introduced by the participants themselves. However, qualitative interviews have limitations. An interview is essentially a verbal report of the subject being discussed, and it is possible that participants do not want to reveal certain things to an interviewer, or that they paint a certain portrait of themselves. As a palliative care nurse, the researcher had previous experience of some of the hospitals that the participants worked in and was aware that some of the interviewees were 
providing partial truths, or trying to paint a more positive picture of the situation than was actually the case. The use of other methods such as observation would allow the researcher a more real insight into the participant's working lives.

In this study, cultural and practical issues, which will be further discussed below, limited the options and the choice of qualitative interviews was a pragmatic choice.

The difficulty in acquiring interview participants has already been discussed within the methodology. This may have led to a bias within the sample towards people who were more sympathetic to research in general, or to the specific topic of this research, palliative care. The cultural and political issues which make research in KSA a unique and, at times difficult, experience may also have affected the content of the interviews. Interviewees may have been fearful of speaking truthfully, despite being given assurances about confidentiality, and they may have feared consequences. It was felt that some of the non-citizens were more open because they were comfortable and willing to talk.

Thematic analysis is a systematic approach to analysing qualitative data. It is relatively descriptive compared to other methods of qualitative analysis, and this allowed analysis and expression of the views of the participants, which was the purpose of this research. To this extent it was seen as important to allow space for the voices of the participants. However, a deeper analysis may have revealed more about why certain things were said. 
Qualitative analysis gives a central role to the researcher. Therefore the interpretation of the data presented must be seen as only one possible interpretation.

\subsection{Reflexivity}

Throughout this study the researcher has endeavoured to practise reflexivity, that is, to reflect upon his own position in relation to the research, and the effects that this may have on this research, in order to better understand and limit the impact of this on the findings.

As a reflexive researcher, he acknowledged his personal beliefs in relation, and these can be found in the methods chapter. These beliefs affected the choice of methodology and method.

Further, he recognised his role at all stages of the research and acknowledged that the accounts heard will be influenced by the questions asked and the interaction between the participant and interviewer during the interview. A researcher must always accept that their interviewee's perceptions of them may influence how much, and what, they reveal about themselves.

Not only does the researcher impact upon the research through their interaction with the participants, but they also bring to the research their own professional persona and other influences, which impact upon the way they interact with the research topic, the participants, and the data. This is highly relevant to the situation in regards to this research. As a palliative care nurse who has worked in KSA, this research was fuelled by the researchers own frustrations at the inadequacy of 
palliative care in KSA, and it is, therefore, essential that this is considered when interpreting the findings. Warren, (2002)recognized the influence of the researchers own biographical position in the findings of qualitative research, in that a researcher may see aspects of themselves in their participants' stories. However, using a descriptive analysis method guarded against interpreting the findings according to the researcher own experiences. By attempting to represent the views of all participants, the researcher was required to represent views which disagreed with his own. An example of this is the views of one female participant regarding the importance of using touch when working with patients, including male patients. While the researcher personally felt her views on this issue were not appropriate for working in KSA, descriptive analysis meant that her views were able to be presented within the findings chapter (p182), without interpreting them through the cultural lens of the researcher.

\subsection{Quality evaluation}

In the methodology chapter, the researcher outlined the broad indicators of quality in qualitative research, suggested by Treharne and Riggs (2014). Transparency is the overarching theme, that is, to be clear exactly how the research has been carried out at each stage. In addition to this the concepts of reflexivity and user involvement, transferability, and triangulation should be considered.

Transparency has been achieved through clear descriptions of the process of the research and endeavouring to represent the views of all participants in the findings. The researcher has ensured reflexivity by reflecting on and acknowledging his role in the research, both in the undertaking and the reporting of the research. End user 
involvement was not a specific aim of this project, due to time and resource constraints. However, the design of the interviews was such that those to whom the research is relevant had the opportunity to contribute and the iterative design of the interviews allowed for checking that researchers understanding was congruent with their intended message. Within the context of palliative care in KSA, giving those involved in palliative care, a voice can be seen as a key progression within the wider context of further research and improvements in this area. The data collection and analysis was undertaken in such a way that it may be possible to apply the findings from this study to KSA as a whole, therefore suggesting transferability

\subsection{Conclusion to the chapter}

This chapter has evaluated the methodological basis for the research, and the methods used to answer the research questions. In addition, the strengths and weaknesses of the study have been outlined. The ability of the researcher to maintain a reflective stance during the research was discussed, and finally, the quality of the research was evaluated and elucidated.

\subsection{Conclusion to the thesis}

The findings of the present study provide a unique and difficult to obtain account of the views of healthcare professionals in KSA, presenting rich information which corroborates and elaborates on previous studies on palliative care in KSA. The present study provides an insight into the views of healthcare workers on palliative care in KSA, significantly adding to this area, substantiating previous research 
aiming to elucidate the issues and also identifying issues that have not been previously covered by research into palliative care in KSA.

Issues not previously explored in the existing literature included the acceptance of healthcare workers in KSA of the family as forming part of the definition of palliative care and incorporating this into practice. Also unique was the finding regarding the importance of the personal characteristics of the individual healthcare worker in providing effective palliative care, including working around inadequate and restrictive policies to ensure the best possible care for the patient. Finally, the need for spiritual services for the patient was raised by participants, which needs to be provided within the specific context of KSA as a conservative Muslim country.

One of the aims of this research was to make recommendations for the future development of palliative care in KSA. These recommendations were made based on the findings, and also viewing the findings within the context of existing literature, as detailed in the discussion. Recommendations for clinical practice were that healthcare professionals providing good palliative care should be encouraged to become leaders in the field, educating others in good practice, and that the emphasis on family in KSA culture should be seen as a positive resource in helping to establish good palliative care. Policy, systems and resources need to be in place to support the development of palliative care throughout KSA, especially to disseminate good practice from the larger to smaller hospitals and rural areas, and training may need to be provided to key members of palliative care staff, in 
positively engaging the media in order to increase public awareness of palliative care.

Undertaking research in KSA is challenging, and researchers working in KSA need a good understanding of the culture and other factors which impact on the potential for research. Training should be provided in research skills, inclusive of ethic matters, to health professionals in Saudi Arabia, particular to native Saudis, who may be in a better position to conduct research. At the same time, it is important for foreigners conducting research in the KSA to be sufficiently briefed about the local culture and ethical norms, for them to be able to develop their data gathering methodologies respecting these norms. Communication with people in Saudi Arabia, as experienced in this study, is subject to a number of cultural protocols, particularly when communicating with a person of the opposite gender. These particularities in the KSA setting were found to cause at least some difficulty in data gathering, which could be avoided in future studies through more careful and culturally sensitive planning.

Due to the scarcity of qualitative research in this area, further qualitative studies would help to illuminate the issues. The areas of the family's role in palliative care, and of spirituality in palliative care in KSA are areas where research in scarce and further research would be beneficial.

This research provides insight into palliative care in KSA, by exploring the views of healthcare professionals, motivated by the researcher's own frustrations at inadequacies of palliative care within KSA. It is not easy to obtain such views in KSA, 
where much is hidden, and people fear that speaking openly may cost them their jobs. This makes the findings even more valuable. It is my sincere hope that they will provide a positive and unique contribution to research on palliative care in KSA and ultimately have a positive impact on the development of palliative care in KSA.

\subsection{The journey's end}

This has been a fascinating journey of discovery and learning for the researcher.

One which began with such enthusiasm and with the full intent of helping bring KSA into line with current world best practice in Palliative Care, and finished with the researcher having a far greater insight into the real issues and current thinking of healthcare workers and policy makers in KSA. The thesis, and the growth of the PhD researcher will add to the KSA specific knowledge base and help ensure the future growth of this very important specialisation. 


\section{References}

Abduh Al-Shaqi, M., Alami, A. H., Al-Zahrani, A. S., Al-Marshad, B., Muammar, A. Bin and Al-shahri, M. Z. (2012) 'The pattern of antimicrobial use for palliative care inpatients during the last week of life.' The American journal of hospice \& palliative care, 29(1) pp. 60-3.

Abdulrahman, K. A. B. (2012) 'The value of medical education research in Saudi Arabia.' Medical Teacher, 34(s1) pp. S1-S3.

Abudari, G., Zahreddine, H., Hazeim, H., Assi, M. A. and Emara, S. (2013) 'palliative care among multinational,' 20.

Abudari, G., Zahreddine, H., Hazeim, H., Assi, M. A. and Emara, S. (2014) 'Knowledge of and attitudes towards palliative care among multinational nurses in Saudi Arabia.' International Journal of Palliative Nursing, 20(9) pp. 435-441.

Ahmed, M. (2012) Kingdom of Saudi Arabia Healthcare Overview [online] [accessed 26th November 2015].

Al-Amri, A. M. (2010) 'Saudi Cancer Patients' Attitudes towards Disclosure of Cancer Information.' Middle East Journal of Cancer, 1(4) pp. 175-180.

Al-Awamer, A. and Downar, J. (2014) 'Developing a palliative care service model for Muslim Middle Eastern countries.' Supportive Care in Cancer, 22(12) pp. 32533262.

Al-Farsy, F. (1990) Modernity and Tradition: The Saudi Equation. London: Routledge. 
Al-Homayan, A. M., Shamsudin, F. M., Subramaniam, C. and Islam, R. (2013)

'Analysis of Health Care System - Resources and Nursing Sector in Saudi Arabia.'

Advances in Environmental Biology, 7(9) pp. 2584-2592.

Al-Jahdali, H., Baharoon, S., Al Sayyari, A. and Al-Ahmad, G. (2012) 'Advance medical directives: a proposed new approach and terminology from an Islamic perspective.' Medicine, Health Care and Philosophy, 16(2) pp. 163-169.

Al-Mazrou, Y. Y. (2002) 'Primary Health Care in Saudi Arabia: Its Development and Future Prospectives.' Journal of Family and Community Medicine, 9(2) pp. 15-16.

Al-Otaibi, A. S. (2014) 'Assessment of Dominant Organisational Cultures Role in Health Care Provision in Riyadh, Saudi Arabia.' Middle Eastern Journal of Scientific Research, 21(10) pp. 1898-1907.

Al-Rowaili, A., Al-aqeel, S. A., Al-Naim, L. S. and Al-Diab, A. I. (2009)

'Appropriateness of cancer pain management in Saudi teaching hospital.' The Gulf Journal of Oncology, (5) pp. 37-43.

Al-Shahri, M. Z. (2002) 'Culturally sensitive caring for Saudi patients.' Journal of Transcultural Nursing, 13(2) pp. 133-138.

Al-Shahri, M. Z. (2002) 'The future of palliative care in the Islamic world.' Western Journal of Medicine, 176(1) pp. 60-61.

Al-Shahri, M. Z. (2009) 'Cancer Pain: Progress and ongoing issues in Saudi Arabia.' Pain Res Manag, 14 pp. 359-60. 
Al-Shahri, M. Z. and Al-Khenaizan, A. (2005) 'Palliative care for Muslim patients.' The Journal of Supportive Oncology, 3 pp. 432-436.

Al-Shahri, M. Z., Brown, S., Ezzat, A. and Khatib, O. (2004) 'Palliative care initiative for the Eastern Mediterranean Region: A proposal.' Annals of Saudi Medicine, 24(6) pp. $465-468$.

Al-Shahri, M. Z., Brown, S. M. and Bruera, E. D. (2004) 'Palliative care: Proposal for a national program in Saudi Arabia.' Saudi Medical Journal, 25(12) pp. 1791-1795.

Al-Shahri, M. Z., Eldali, A. M. and Al-Zahrani, O. (2012) ‘Nonpain Symptoms of New and Follow-up Cancer Patients Attending a Palliative Care Outpatient Clinic in Saudi Arabia.' Indian Journal of Palliative Care, 18(2) pp. 98-102.

Al-Shahri, M. Z., Sroor, M. Y. and Alsirafy, S. A. (2010) 'The impact of implementing referral criteria on the pattern of referrals and admissions to a palliative care program in Saudi Arabia.' The Journal of Supportive Oncology, 8(2) pp. 78-81.

Al-Sheikh, E. M. (1995) An analysis of the difficulties involved in introducing western programmes for the training of health care professionals into the Kingdom of Saudi Arabia. PhD Swansea University.

Al-Zahrani, A. S., El-Kashif, A. T., Haggag, R. M. and Alsirafy, S. A. (2012) ‘Pattern of Cancer Deaths in a Saudi Tertiary Care Hospital.' American Journal of Hospice and Palliative Medicine, 30(1) pp. 21-24.

Al-Zahrani, O., Eldali, A. and Al-shahri, M. Z. (2014) 'Prevalence and severity of pain in cancer patients in an outpatient palliative care setting in Saudi Arabia.' Qatar 
Medical Journal, 2014 pp. 38-45.

Alamri, S. (2012) 'Knowledge of the residents at King Abdul-Aziz University Hospital (KAAUH) about palliative care.' Journal of Family and Community Medicine, 19(3) p. 194.

Albaqawi, H. (2015) 'Pain Management in Hail Region Hospitals in Saudi Arabia.' In Paper presented at 4th International Conference on Nursing and Healthcare. San Francisco USA, October 5-17 2015.

Aldosh, A. (2015) Designing a practice-based, culturally sensitive model of healthpatient education for hospital nurses to use in Saudi Arabia. Ph.D. University of Salford.

Alhirz, H. and Sajeev, A. S. M. (2015) 'Do cultural dimensions differentiate ERP acceptance? A study in the context of Saudi Arabia.' Information Technology \& People. Emerald Group Publishing Limited, 28(1) pp. 163-194.

Alhusaini, H. A. (2006) Obstacles to the efficiency and performance of Saudi nurses at the Ministry of Health, Riyadh Region: analytical field study. Riyadh, Saudi Arabia: Ministry of Health.

Aljawi, D. M. and Harford, J. B. (2012) 'Palliative Care in the Muslim-Majority Countries: The Need for More and Better Care.' In Chang, E. and Johnson, A. (eds) Contemporary and Innovative Practice in Palliative Care. New York: InTech, pp. 137150.

Alkabba, A. F., Hussein, G. M. A., Albar, A. A., Bahnassy, A. A. and Qadi, M. (2012) 
'The major medical ethical challenges facing the public and healthcare providers in Saudi Arabia.' Journal of Family and Community Medicine, 19(1) pp. 1-6.

Almalki, M., Fitzgerald, G. and Clark, M. (2011) ‘Health care system in Saudi Arabia: An overview.' Eastern Mediterranean Health Journal, 17(10) pp. 784-793.

Almobarak, F. (2014) 'Exploring the perspectives of nurses, physicians, and healthcare administrators in Saudi Arabian hospitals on palliative care and palliative care nursing.' Journal of Health Specialties, 2(2) p. 54.

Almuzaini, A. S., Salek, M. S. and Nicholls, P. J. (1998) 'The attitude of health care professionals toward the availability of hospice services for cancer patients and their carers in Saudi Arabia.' Palliative Medicine, 6591(98) pp. 365-373.

Alshaikh, Z. and Sormunen, T. (2015) 'Nurses ' Knowledge about Palliative Care in an Intensive Care Unit in Saudi Arabia.' Middle Eastern Journal of Nursing, 9(1) pp. $7-13$.

Alshammary, S., Alsuhail, A., Duraisamy, B., Ratnapalan, S. and Alabdullateef, S. (2015) 'Interval between first palliative care consultation and death among patients in a comprehensive cancer center in Saudi Arabia.' Journal of Health Specialties, 3(2) p. 61.

Alshammary, S., Anbar, M., Duraisamy, B. and Abdullah, A. (2014) 'Palliative care in Saudi Arabia: Two decades of progress and going strong.' Journal of Health Specialties, 2(2) p. 59.

Alsirafy, S. A., Abou-Alia, A. M. and Ghanem, H. M. (2013) ‘Palliative Care 
Consultation Versus Palliative Care Unit: Which Is Associated With Shorter Terminal Hospitalization Length of Stay Among Patients With Cancer?' The American Journal of Hospice \& Palliative Care, 32(3) pp. 275-279.

Alsirafy, S. A., Hassan, A. A. and Al-Shahri, M. Z. (2009) ‘Hospitalization pattern in a hospital-based palliative care program: an example from Saudi Arabia.' The American Journal of Hospice \& Palliative care, 26(1) pp. 52-6.

Alsirafy, S. A., Sroor, M. Y. and Al-Shahri, M. Z. (2010) 'Referral timing of in-hospital cancer deaths to palliative care in a Saudi tertiary care hospital.' Progress in Palliative Care, 18(2) pp. 85-88.

AlYateem, S. and Al-Yateem, N. (2014a) 'the Experience of Overseas Nurses Caring for Muslim Patients in Kingdom of Saudi Arabia and Uae: a Qualitative Study.' International Journal of Research in Nursing, 5(1) pp. 17-24.

AlYateem, S. and Al-Yateem, N. (2014b) 'The Experience of Overseas Nurses Caring for Muslim Patients in Kingdom of Saudi Arabia and UAE: a Qualitative Study.' International Journal of Research in Nursing, 5(1) pp. 17-24.

Amoah, C. F. (2011) 'The central importance of spirituality in palliative care.' International Journal of Palliative Nursing, 17(7) pp. 353-359.

Asgeirsdottir, G. H., Sigurbjörnsson, E., Traustadottir, R., Sigurdardottir, V., Gunnarsdottir, S. and Kelly, E. (2013) "To Cherish Each Day as it Comes": a qualitative study of spirituality among persons receiving palliative care.' Supportive Care in Cancer, 21(5) pp. 1445-1451. 
Babgi, A. A. (2009) 'Legal issues in end-of-life care: perspectives from Saudi Arabia and the United States.' The American journal of hospice \& palliative care, 26(2) pp. 119-27.

Babgi, A. A. (2010) ‘Pain Coping Behaviors of Saudi Patients Suffering from Advanced Cancer: A Revisited Experience,' 11 pp. 103-106.

Bahammam, F. S. (2012) The New Muslim Guide. Riyadh, Saudi Arabia: King Fahd National Library Cataloging-in-Publication Data.

Baines, M. (2010) 'The origins and development of palliative care at home.' Progress in Palliative Care, 18(1) pp. 4-8.

Basic Law of Governance (1992) Kingdom of Saudi Arabia Bureau of Experts at the Council of Ministers.

Beuken, V. Den, Everdingen, M., De Rijke, J., Kessels, A. and Schouten, H. (2007) 'Prevalence of pain in patients with cancer: a systematic review of the past 40 years.' Ann Oncol, 18(9) pp. 1437-1449.

Bjerke, B. and Al-Meer, A. (1993) 'Culture's consequences: Management in Saudi Arabia.' Leadership \& Organization Development Journal. MCB UP Ltd, 14(2) pp. 3035.

Boseley, S. (2014) 'End of life care requires "widescale improvements”, report says.' The Guardian. May.

Bou Khalil, R. (2013) 'Attitudes, beliefs and perceptions regarding truth disclosure of 
cancer-related information in the Middle East: a review.' Palliative \& supportive care, $11(1)$ pp. 69-78.

Braun, V. and Clarke, V. (2006) 'Using thematic analysis in psychology.' Qualitative Research in Psychology, 3(2) pp. 77-101.

Broback, G. and Bertero, C. (2003) 'How next of kin experience palliative care of relatives at home.' European Journal of Cancer Care, 12(4) pp. 193-199.

Burbank, P. M. and Martins, D. C. (2010) 'Symbolic interactionism and critical perspective: divergent or synergistic?' Nursing Philosophy, 11(1) pp. 25-41.

Central Department of Statistics and Information (2015) Latest Statistical Releases [Online] [Accessed 25th December 2015].

Charmaz, K. (1990) “Discovering” chronic illness: using grounded theory.' Social Science Medicine, 30(11) pp. 1161-1172.

Charmaz, K. (2006) Constructing Grounded Theory: a practical guide through qualitative analysis. London: Sage.

Charon, J. M. (2004) Symbolic Interactionism: An Introduction, an Interpretation, an Integration. Upper Saddle River: Pearson Prentice Hall.

Choong, K. A. (2015) 'Islam and palliative care.' Global Bioethics, 26(1) pp. 28-42.

Clark, D. (2004) 'Total pain': the work of Cicely Saunders and the maturing of a concept. 25th September. University of Glasgow End of life studies [Online] [Accessed 16th February 2015]. 
Clark, D. and Centeno, C. (2014) 'Historical perspectives in End-of-Life care in Global context.' In Lazenby, M., McCorkle, R., and Sulmasy, D. P. (eds) Safe Passage: A global spiritual sourcebook for care at the end of life. Oxford: Oxford University Press.

Clark, D. and Seymour, J. (1999) Reflections of palliative care: sociological and policy perspectives. Buckingham: Open University Press.

Cohen, A. B. and Koenig, M. D. (2002) 'Spirituality in Palliative Care.' Geriatric Times, 3(6) pp. 25-26.

Corbin, J. and Strauss, A. (2008) Basics of Qualitative Research: Procedures for developing grounded theory. Los Angeles: Sage.

Denzin, N. K. and Lincoln, Y. S. (2011) The Sage Handbook of Qualitative Research. California: Sage.

Department of Health (2008) End of Life Care Strategy: promoting high-quality care for adults at the end of their life. 9840.

DeVito, J. (1992) The Interpersonal Communication Book. New York: Harper Collins.

Dulayami, S. T. H. and Robinson, L. (2015) 'The individual and the collective.' Journal of Documentation. Emerald Group Publishing Limited, 71(1) pp. 198-209.

Etherington, K. (2004) Becoming a Reflexive Researcher - Using Our Selves in Research. London: Jessica Kinsley Publications.

Fakeeh, M. S. (2010) Saudization as a Solution for Unemployment The Case of 
Jeddah Western Region. Ph.D. University of Glasgow.

Fallon, M. and Foley, P. (2012) 'Rising to the challenge of palliative care for nonmalignant disease.' Palliative Medicine, 26(2) pp. 99-100.

Falquero, A. (2016) 'Information about Saudi Arabia.' Trotmate, [Online]([Accessed on 1st February 2016]) p. http://www.trotmate.com/countries/67-saudi-arabia/.

Fertuck, E. A. (2007) 'Review of evidence-based psychotherapy: Where theory and practice meet.' Psychotherapy: Theory, Research, Practice, Training, 44(1) pp. 115116.

Garrido, M. M., Idler, E. L., Leventhal, H. and Carr, D. (2012) ‘Pathways From Religion to Advance Care Planning: Beliefs About Control Over Length of Life and End-of-Life Values.' The Gerontologist, 53(5) pp. 801-816.

Gatrad, A. R. and Sheikh, A. (2002) 'Palliative care for Muslims and issues before Death.' International Journal of Palliative Nursing, 8(11) pp. 526-31.

Ghanem, H. M., Rawabi, M. S., Abou Alia, A. M., Al-Zayir, A. S. and Alsirafy, S. A. (2011) 'Pattern of Referral on Noncancer Patients to Palliative Care in the Eastern Province of Saudi Arabia.' Indian Journal of Palliative Care, 17(3) pp. 235-237.

Glaser, B. G. and Strauss, A. L. (1967) The Discovery of Grounded Theory: Strategies for Qualitative Research. Chicago: Aldine Publishing Company.

Gouda, A., Al-Jabbary, A. and Fong, L. (2010) 'Compliance with DNR policy in a tertiary care center in Saudi Arabia.' Intensive Care Medicine, 36(12) pp. 2149- 
2153.

Gray, A. J., Ezzat, A. and Volker, S. (1995a) ‘Developing palliative care services for terminally ill cancer patients in Saudi Arabia.' Ann Saudi Med, 15 pp. 370-7.

Gray, A. J., Ezzat, A. and Volker, S. (1995b) 'Special Communication,' 15(4).

Holliday, A. (2007) Doing and writing qualitative research. London: Sage Publications.

Hui, D., Elsayem, A., De La Cruz, M., Berger, A., Zhukovsky, D. S., Palla, S., Evans, A., Fadul, N., Palmer, J. L. and Bruera, E. (2010) 'Availability and Integration of Palliative Care at US Cancer.' Journal of the American Medical Association, 303(11) pp. 10541061.

Ibrahim, N. a, Björnsdottir, I., Alwan, A. S. Al and Honore, P. H. (2013) 'Insights about health-related quality of life in cancer patients indicate demands for better pharmaceutical care.' Journal of oncology pharmacy practice : official publication of the International Society of Oncology Pharmacy Practitioners.

Isbister, W. H. and Bonifant, J. (2001) 'Implementation of the World Health Organization "analgesic ladder" in Saudi Arabia.' Palliative Medicine, 15(2) pp. 135140.

Jazieh, A., Bamefleh, H., Demirkazik, A., Gaafar, R. M., Geara, F. B., Javaid, M., Khader, J., Khodadad, K., Omar, W., Saadeddin, A., Sabe, H. Al, Shadmehr, M. B., El Sherif, A., Uddin, N., Jahanzeb, M. and Ettinger, D. (2010) 'Modification and implementation of NCCN guidelines on non-small cell lung cancer in the Middle East 
and North Africa region.' Journal of the National Comprehensive Cancer Network: JNCCN, 8 Suppl 3(July) pp. S16-S21.

Jocham, H. R., Dassen, T., Widdershoven, G. and Halfens, R. (2006) 'Quality of life in palliative cancer care patients: a literature review.' Journal of Clinical Nursing, 15 pp. 1188-1195.

Karim, S., Zekri, J., Abdelghany, E., Dada, R., Munsoor, H. and Ahmad, I. (2015) 'Time from last chemotherapy to death and its correlation with the end of life care in a referral hospital.' Indian Journal of Medical and Paediatric Oncology, 36(1) p. 55.

Kellehear, A. (2000) 'Spirituality and palliative care: a model of needs.' Palliative Medicine, 14(2) pp. 149-155.

Kuwait News Agency (2014) Kuwait has world's largest palliative care center [Online] [Accessed on 16th February 2016].

Lietz, C. A., Langer, C. L. and Furman, R. (2006) 'Establishing Trustworthiness in Qualitative Research in Social Work: implications from a study regarding spirituality.' Qualitative Social Work, 5(4) pp. 135-156.

Lincoln, Y. S. and Guba, E. G. (1985) Naturalistic Inquiry. Thousand Oaks CA: Sage. Lynch, T., Clark, D., Centeno, C., Rocafort, J., Flores, L. A., Greenwood, A., Praill, D., Brasch, S., Giordano, A., De Lima, L. and Wright, M. (2009) 'Barriers to the development of palliative care in the countries of Central and Eastern Europe and the Commonwealth of Independent States.' Journal of Pain and Symptom 
Management, 37(3) pp. 305-15.

Malloy, P., Paice, J., Coyle, N., Coyne, P., Smith, T. and Ferrell, B. (2014) 'Promoting Palliative Care Worldwide Through International Nursing Education.' Journal of Transcultural Nursing, 25(4) pp. 410-417.

Mathew, A., Cowley, S., Bliss, J. and Thistlewood, G. (2003) 'The development of palliative care in national government policy in England, 1986-2000.' Palliative Medicine, 17 pp. 270-282.

Merriam, S. B. (2009) Qualitative Research: A Guide to Design and Implementation. San Francisco: John Wiley \& Sons.

Milligan, S. and Potts, S. (2009) 'The history of palliative care.' In Stevens, E., Jackson, S., and Milligan, S. (eds) Palliative Nursing: Across the Spectrum of Care. Blackwell, pp. 5-16.

Mills, J., Bonner, A. and Francis, K. (2006) 'The Development of Constructivist Grounded Theory.' International Journal of Qualitative Methods, 5(1) p. Article 3. Ministry of Health (2009) 'Statistics Yearbook. Saudi Arabia.' Saudi Arabia: Ministry of Health.

Ministry of Health (2013) 'Achievements Book 2013. Saudi Arabia.' Saudi Arabia: Ministry of Health.

Mir, T. (2011) 'Care of the terminally ill from religious perspectives: Role of palliative and hospice care.' Journal of the Islamic Association of North America, 43 
pp. 168-172.

von Mittelstaedt, J. and Shafy, S. (2015) 'Lifting the Veil: How Working Women Are Remaking Saudi Arabia.' Spiegel Online International. June.

MOH Catalonia (1995) Barcelona Declaration. Spain.

Murphy, E., Dingwall, R., Greatbatch, D., Parker, S. and Watson, P. (1998)

'Qualitative research methods in health technology assessment: a review of the literature.' Health Technology Assessment, 2(1) p. 274.

National Council for Palliative Care (2011) Commissioning End of Life Care.

NationMaster (2016) Religion>Islam>Percentage Muslim: Countries Compared.

NICE (2004) Guidance on cancer services: improving supportive and palliative care for adults. London.

Nixon, A. (2003) 'Palliative Care in Saudi Arabia : A Brief History.' Journal of pain and palliative care, $17(3 / 4)$ pp. 45-49.

National Institute for Clinical Excellence (2004) Improving supportive and palliative care for adults with cancer.

Nyatanga, B. (2002) 'Culture, palliative care and multiculturalism.' International Journal of Palliative Nursing, 8(5) pp. 240-246.

Organization of Petroleum Exporting Countries (2016) Saudi Arabia Facts and Figures. 
Pope, C. and Mays, N. (1996) 'Quality in Qualitative Health Research.' In Qualitative Research in Health Care. London: Blackwell.

Puchalski, C. M. (2012) 'Spirituality as an essential domain of palliative care: Caring for the whole person.' Progress in Palliative Care, 20(2) pp. 63-65.

Quandl (2016) Saudi Arabia Economy Data.

Reynolds, J., Kizito, J., Ezumah, N., Mangesho, P., Allen, E. and Chandler, C. (2011) 'Quality assurance of qualitative research: a review of the discourse.' Health Research Policy and Systems. BioMed Central Ltd, 9(1) p. 43.

van Rooyen, D., Telford-Smith, C. D. and Strümpher, J. (2010) 'Nursing in Saudi Arabia: reflections on the experiences of South African nurses.' Health SA Gesondheid, 15(1) pp. 1-9.

Royal Embassy of Saudi Arabia (2015) About Saudi Arabia: the health care network. Royal Embassy of Saudi Arabia (2016) About Saudi Arabia.

Sandelowski, M. (2015) 'A Matter of Taste: evaluating the quality of qualitative research.' Nursing Inquiry, 22(2) pp. 86-94.

Sandelowski, M., Docherty, S. and Emden, C. (1997) 'Focus on qualitative methods. Qualitative meta synthesis: issues and techniques.' Research in Nursing and Health, 20 pp. 365-71.

Saudi Commission for Tourism and National Heritage (2013) Saudi History: Establishment of Saudi Arabia. SaudiTourism.sa. 
Sayeed, S., Padela, a., Naim, M. Y. and Lantos, J. D. (2012) ‘A Saudi Family Making End-of-Life Decisions in the PICU.' Pediatrics, 129(4) pp. 764-768.

Schimmel, A. (1992) Islam. An Introduction. Albany, US: State University of New York Press.

Schultz, M., Baddarni, K. and Bar-Sela, G. (2012) 'Reflections on palliative care from the Jewish and Islamic tradition.' Evidence-based complementary and alternative medicine : eCAM, 2012, January, p. 693092.

Sebai, Z. A., Milaat, W. A. and Al-Zulaibani, A. A. (2001) ‘HEALTH CARE SERVICES IN SAUDI ARABIA: PAST, PRESENT AND FUTURE.' Journal of Family and Community Medicine, 8(3) pp. 19-23.

Shakir, Z. (2008) 'Men, women and the balance of power.' Seasons: The Journal of the Zaytuna Institute, 5(1) pp. 81-91.

Sidumo, E. M., Ehlers, V. J. and Hattingh, S. P. (2010) 'Cultural knowledge of nonMuslim nurses working in Saudi Arabian obstetric units.' Curationis, 33(September 2010) pp. 48-55.

Smith, J. A., Flowers, P. and Larkin, M. (2009) Interpretive Phenomenological Analysis. London: Sage.

Spencer, L., Ritchie, J., Lewis, J., Dillon, L. and Research, N. C. for S. (2003) Quality in qualitative evaluation: A framework for assessing research evidence.

Stjernswärd, J., Foley, K. M. and Ferris, F. D. (2007) 'The Public Health Strategy for 
Palliative Care.' Journal of Pain and Symptom Management, 33(5) pp. 486-493.

Tayeb, M. A. (2010) 'A "good death": perspectives of Muslim patients and health care providers.' Ann Saudi Med, 30(3) pp. 215-221.

The Economist (2015) 'The 2015 Quality of Death Index Ranking palliative care across the world.'

The Islam Project (2016) What is Shariah? Major Sources and Principles of Islamic Law.

The World Bank (2011) World Development Report 2011. Wdi 2011.

Tracey, S. j (2010) ‘Qualitative Quality: Eight “Big-Tent” Criteria for Excellent Qualitative Research.' Qualitative Inquiry, 16(10) pp. 837-851.

Treharne, G. J. and Riggs, D. W. (2014) 'Ensuring quality in qualitative research.' In Qualitative Research in Clinical and Health Psychology.

Trenwith, C. (2015) 'Saudi Arabia suspects $\$ 266 \mathrm{~m}$ worth of “corruption” related to MERS.' Arabian business. January.

Twinn, S. (1997) 'An exploratory study examining the influence of translation on the validity and reliability of qualitative data in nursing research.' Journal of advanced nursing, 26(2) pp. 418-423.

United Nations Statistics Divisions (2015) undata saudi arabia.

Vachon, M. L. S., Kristjanson, L. and Higginson, I. (1995) 'Psychosocial issues in palliative care: The patient, the family, and the process and outcome of care.' 
Journal of pain and symptom management, 10(2) pp. 142-149.

Warren, C. A. B. (2002) 'Qualitative Interviewing.' In Handbook of Interview Research. Context and Method. London: Sage, pp. 83-101.

Webster, R., Lacey, J. and Quine, S. (2007) 'Palliative care: a public health priority in developing countries.' Journal of public health policy, 28(1) pp. 28-39.

Wee, B. and Jamison, H. (2014) 'Invited Speaker Abstract.' In The 10th Palliative Care Conference, Harrogate, July 12-14, p. 2014.

WHO (2015) World Health Organisation Saudi Arabia.

Wolcott, H. F. (1990) 'Writing up qualitative research.' Qualitative Research Methods, 20 p. 94.

World Health Organisation (2013) Country Cooperation Strategy at a Glance: Saudi Arabia.

World Health Organisation (2015) WHO definition of palliative care.

World Medical Association, 64thWMAGeneral Assembly, Fortaleza, B. (2013) 'World Medical Association Declaration of Helsinki Ethical Principles for Medical Research Involving Human Subjects.' Jama, 310(20) pp. 2191-4.

Wright, M., Wood, J., Lynch, T. and Clark, D. (2008) ‘Mapping Levels of Palliative Care Development: A Global View.' Journal of Pain and Symptom Management, 35(5) pp. 469-485.

Yardley, L. (2000) 'Dilemmas in qualitative health research.' Psychology and Health, 
15 pp. 215-228.

Younge, D., Moreau, P., Ezzat, A. and Gray, A. (1997) 'Communicating with Cancer Patients in Saudi Arabia.' Annals of the New York Academy of Sciences, 809(1 Communication) pp. 309-316.

Youssef, H. A. M., Mansour, M. A. M., Al-Zahrani, S. S., Ayasreh, I. R. A. and K, A. E.K. R. A. (2015) ‘Prioritizing Palliative Care: Assess Undergraduate Nursing Curriculum , Knowledge and Attitude among Nurses Caring End-of-Life Patients,' European J (August) pp. 90-101.

Zeinah, G. F. A., Al-Kindi, S. G. and Hassan, A. A. (2013) 'Middle East experience in palliative care.' The American journal of hospice \& palliative care, 30(1) pp. 94-9. 
Appendices 
Appendix 1: KSA literature table 


\begin{tabular}{|c|c|c|c|c|c|c|c|c|c|}
\hline No. & \begin{tabular}{|l|l} 
Journal \\
\end{tabular} & Paper & Date & Key results & Methods & $\begin{array}{l}\text { Sample } \\
\text { Size }\end{array}$ & $\begin{array}{l}\text { Volu } \\
\text { me }\end{array}$ & $\begin{array}{l}\text { Pag } \\
\text { es }\end{array}$ & $\begin{array}{l}\text { Place of } \\
\text { Publicati } \\
\text { on }\end{array}$ \\
\hline 1 & $\begin{array}{l}\text { Pain Research } \\
\text { \& } \\
\text { Management } \\
\text { The Journal of } \\
\text { the Canadian } \\
\text { Pain Society }\end{array}$ & $\begin{array}{l}\text { Cancer pain: } \\
\text { progress and } \\
\text { ongoing } \\
\text { issues in } \\
\text { Saudi Arabia } \\
\text { by Al-Shahri, } \\
\text { Mohammad } \\
\text { Zafir }\end{array}$ & 09/2009 & Review article & Review article & $\begin{array}{l}\text { Review } \\
\text { article }\end{array}$ & 14 & 359 & Canada \\
\hline 2 & $\begin{array}{l}\text { The Western } \\
\text { journal of } \\
\text { medicine }\end{array}$ & $\begin{array}{l}\text { The future } \\
\text { of palliative } \\
\text { care in the } \\
\text { Islamic } \\
\text { world. } \\
\text { by Al-Shahri, } \\
\text { Mohammad }\end{array}$ & $01 / 2002$ & Review article & Review article & $\begin{array}{l}\text { Review } \\
\text { article }\end{array}$ & 176 & 60 & $\begin{array}{l}\text { United } \\
\text { States }\end{array}$ \\
\hline 3 & $\begin{array}{l}\text { The journal of } \\
\text { supportive } \\
\text { oncology }\end{array}$ & $\begin{array}{l}\text { Palliative } \\
\text { care for } \\
\text { Muslim } \\
\text { patients. } \\
\text { by al-Shahri, } \\
\text { Mohammad } \\
\text { Zafir and al- } \\
\text { Khenaizan, } \\
\text { Abdullah } \\
\end{array}$ & $11 / 2005$ & How We Do It & How We Do It & $\begin{array}{l}\text { How We } \\
\text { Do It }\end{array}$ & 3 & 432 & $\begin{array}{l}\text { United } \\
\text { States }\end{array}$ \\
\hline
\end{tabular}




\begin{tabular}{|c|c|c|c|c|c|c|c|c|c|}
\hline 4 & $\begin{array}{l}\text { Saudi medical } \\
\text { journal }\end{array}$ & $\begin{array}{l}\text { Palliative } \\
\text { care. } \\
\text { Proposal for } \\
\text { a national } \\
\text { program in } \\
\text { Saudi Arabia } \\
\text { by Al-Shahri, } \\
\text { Mohammad } \\
\text { Z; Brown, } \\
\text { Stuart M; } \\
\text { Bruera, } \\
\text { Eduardo D }\end{array}$ & $12 / 2004$ & Editorial & Editorial & Editorial & 25 & $\begin{array}{l}179 \\
1\end{array}$ & $\begin{array}{l}\text { Saudi } \\
\text { Arabia }\end{array}$ \\
\hline 5 & $\begin{array}{l}\text { Indian Journal } \\
\text { of palliative } \\
\text { care }\end{array}$ & $\begin{array}{l}\text { Nonpain } \\
\text { Symptoms } \\
\text { of New and } \\
\text { Follow-up } \\
\text { Cancer } \\
\text { Patients } \\
\text { Attending a } \\
\text { Palliative } \\
\text { Care } \\
\text { Outpatient } \\
\text { Clinic in } \\
\text { Saudi Arabia } \\
\text { by Al-Shahri, } \\
\text { Mohammad } \\
\text { Zafir; Eldali, } \\
\text { Abdelmonei } \\
\text { m M; Al- } \\
\text { Zahrani, } \\
\text { Omar }\end{array}$ & 2012 & $\begin{array}{l}\text { The most common cancer } \\
\text { is female breast ( } 27.4 \%) \\
\text { followed by head and neck } \\
\text { ( } 15.3 \%) \text {. Majority of } \\
\text { patients ( } 67 \% \text { ) were new } \\
\text { to PC clinic. Patients had } \\
5.1 \text { nonpain symptoms on } \\
\text { average, with most } \\
\text { common symptoms being } \\
\text { tiredness ( } 79.8 \%) \text { loss of } \\
\text { appetite ( } 71.8 \%) \text {, dry } \\
\text { mouth (69.4\%), anxiety } \\
\text { (60.5\%), and depression } \\
\text { ( } 50.8 \%) \text {. The least } \\
\text { common symptoms were } \\
\text { confusion and nausea } \\
\text { ( } 22.6 \% \text { each). The median } \\
\text { scores of severity were } \\
\text { highest for tiredness, loss } \\
\text { of appetite, dry mouth, }\end{array}$ & $\begin{array}{l}\text { Over a five months } \\
\text { period, consecutive } \\
\text { adult cancer patients } \\
\text { attending PC outpatient } \\
\text { clinic at a tertiary } \\
\text { hospital were evaluated } \\
\text { for the presence and } \\
\text { severity of } 10 \text { nonpain } \\
\text { symptoms. Patients } \\
\text { were grouped to new or } \\
\text { follow-up cases and } \\
\text { were also grouped } \\
\text { according to } \\
\text { performance status and } \\
\text { cancer type. Prevalence } \\
\text { and severity of } \\
\text { symptoms were } \\
\text { compared between } \\
\text { groups using t-test or }\end{array}$ & $\begin{array}{l}\text { Fifty- } \\
\text { one } \\
\text { males } \\
\text { and } 73 \\
\text { females }\end{array}$ & 18 & $\begin{array}{l}98- \\
102\end{array}$ & India \\
\hline
\end{tabular}




\begin{tabular}{|c|c|c|c|c|c|c|c|c|c|}
\hline & & & & $\begin{array}{l}\text { and insomnia ( } 5 \text { points } \\
\text { each). Symptoms were } \\
\text { fewer among patients with } \\
\text { good performance status } \\
\text { ( } P=0.002 \text { ), whereas age, } \\
\text { gender, cancer type, and } \\
\text { encounter type were not } \\
\text { associated with difference } \\
\text { in symptom prevalence. } \\
\text { Younger patients, females } \\
\text { and those with poor } \\
\text { performance status have } \\
\text { shown a tendency toward } \\
\text { higher severity scores for } \\
\text { several symptoms. }\end{array}$ & $\begin{array}{l}\text { analysis of variance as } \\
\text { appropriate. }\end{array}$ & & & & \\
\hline 6 & $\begin{array}{l}\text { The journal of } \\
\text { supportive } \\
\text { oncology }\end{array}$ & $\begin{array}{l}\text { The impact } \\
\text { of } \\
\text { implementin } \\
\mathrm{g} \text { referral } \\
\text { criteria on } \\
\text { the pattern } \\
\text { of referrals } \\
\text { and } \\
\text { admissions } \\
\text { to a } \\
\text { palliative } \\
\text { care } \\
\text { program in } \\
\text { Saudi Arabia } \\
\text { by Al-Shahri, } \\
\text { Mohammad } \\
\text { Z; Sroor, }\end{array}$ & $03 / 2010$ & $\begin{array}{l}\text { During the two study } \\
\text { periods, the palliative care } \\
\text { unit (PCU) received } 620 \\
\text { admissions related to } 518 \\
\text { patients. Of them, } 314 \\
\text { ( } 50.6 \% \text { ) were in the } \\
\text { precriteria period. A total } \\
\text { of } 1,216 \text { new cancer } \\
\text { referrals were received by } \\
\text { the PCP during the study } \\
\text { period, with } 53.2 \% \\
\text { received during the } \\
\text { precriteria period. The } \\
\text { postcriteria period was } \\
\text { associated with a } \\
\text { proportional increment in } \\
\text { admissions to the PCU } \\
\text { through the emergency }\end{array}$ & $\begin{array}{l}\text { Two periods, } 19 \text { months } \\
\text { each, were identified: } \\
\text { one before the } \\
\text { implementation of the } \\
\text { referral criteria } \\
\text { (precriteria), and the } \\
\text { other started six months } \\
\text { later (post-criteria). }\end{array}$ & $\begin{array}{l}518 \\
\text { patients }\end{array}$ & 8 & 78 & $\begin{array}{l}\text { United } \\
\text { States }\end{array}$ \\
\hline
\end{tabular}




\begin{tabular}{|c|c|c|c|c|c|c|c|c|c|}
\hline & & $\begin{array}{l}\text { Mahmoud Y; } \\
\text { Alsirafy, } \\
\text { Samy A }\end{array}$ & & $\begin{array}{l}\text { room }(\mathrm{P}<0.001) \text { by the } \\
\text { palliative care team }(\mathrm{P}= \\
0.001) \text { for patients whose } \\
\text { care had been previously } \\
\text { transferred completely to } \\
\text { the } \mathrm{PCP} \text {. The median } \\
\text { survival of referred } \\
\text { patients decreased from } \\
2.27 \text { months during the } \\
\text { precriteria period to } 1.73 \\
\text { months during the } \\
\text { postcriteria period }(\mathrm{P}= \\
0.0051) \text {. }\end{array}$ & & & & & \\
\hline 7 & $\begin{array}{l}\text { The American } \\
\text { journal of } \\
\text { hospice \& } \\
\text { palliative care }\end{array}$ & $\begin{array}{l}\text { Hospitalizati } \\
\text { on pattern } \\
\text { in a hospital- } \\
\text { based } \\
\text { palliative } \\
\text { care } \\
\text { program: an } \\
\text { example } \\
\text { from Saudi } \\
\text { Arabia } \\
\text { by Alsirafy, } \\
\text { Samy A; } \\
\text { Hassan, Azza } \\
\text { A; Al-Shahri, } \\
\text { Mohammad } \\
\text { Z }\end{array}$ & $02 / 2009$ & $\begin{array}{l}\text { Of all admissions, } 66 \% \\
\text { were hospitalized through } \\
\text { the emergency room. The } \\
\text { average hospital stay was } \\
24 \text { days. The majority } \\
\text { ( } 86 \% \text { ) of patients died in- } \\
\text { hospital }\end{array}$ & retrospective review & 629 & 26 & 52 & $\begin{array}{l}\text { United } \\
\text { States }\end{array}$ \\
\hline
\end{tabular}




\begin{tabular}{|c|c|c|c|c|c|c|c|c|c|}
\hline 8 & $\begin{array}{l}\text { Annals of the } \\
\text { New York } \\
\text { Academy of } \\
\text { Sciences }\end{array}$ & $\begin{array}{l}\text { Communicat } \\
\text { ing with } \\
\text { cancer } \\
\text { patients in } \\
\text { Saudi Arabia } \\
\text { by Younge, } \\
\text { D; Moreau, } \\
\text { P; Ezzat, A; } \\
\text { Gray, A }\end{array}$ & $02 / 1997$ & $x$ & $x$ & $x$ & 809 & $\begin{array}{l}309 \\
- \\
316\end{array}$ & $\begin{array}{l}\text { United } \\
\text { States }\end{array}$ \\
\hline 9 & $\begin{array}{l}\text { Palliative } \\
\text { Medicine }\end{array}$ & $\begin{array}{l}\text { The attitude } \\
\text { of } \\
\text { healthcare } \\
\text { professional } \\
\text { s toward the } \\
\text { availability } \\
\text { of hospice } \\
\text { services for } \\
\text { cancer } \\
\text { patients and } \\
\text { their carers } \\
\text { in Saudi } \\
\text { Arabia } \\
\text { by } \\
\text { Almuzaini, A } \\
\text { S; Salek, M } \\
\text { S; Nicholls, P } \\
\text { J; Alomar, B } \\
\text { A }\end{array}$ & 07/1998 & $\begin{array}{l}\text { the level of cancer care in } \\
\text { the Ministry of Health } \\
\text { hospitals was poor } \\
\text { compared to other } \\
\text { hospitals such as the King } \\
\text { Faisal Specialist Hospital } \\
\text { and Research Centre, } \\
\text { military hospitals or } \\
\text { university hospitals in the } \\
\text { kingdom, perhaps due to } \\
\text { the absence of home care } \\
\text { services or periodic follow- } \\
\text { up by their regional } \\
\text { hospitals. It was also } \\
\text { reported that the shortage } \\
\text { of drugs used in cancer } \\
\text { management, the severe } \\
\text { restriction of prescribing } \\
\text { narcotic analgesics and } \\
\text { lack of cancer care } \\
\text { knowledge were the major } \\
\text { impediments to providing } \\
\text { good cancer care. }\end{array}$ & questionnaire & $\begin{array}{l}\text { Six- } \\
\text { hundred } \\
\text {-and- } \\
\text { ninety- } \\
\text { five } \\
\text { particip } \\
\text { ants } \\
\text { (136 } \\
\text { cancer } \\
\text { patients } \\
\text {, } 161 \\
\text { informal } \\
\text { carers, } \\
\text { and 398 } \\
\text { healthca } \\
\text { re } \\
\text { professi } \\
\text { onals) }\end{array}$ & 12 & $\begin{array}{l}365 \\
- \\
373\end{array}$ & $\begin{array}{l}\text { United } \\
\text { Kingdo } \\
\mathrm{m}\end{array}$ \\
\hline
\end{tabular}




\begin{tabular}{|c|c|c|c|c|c|c|c|c|c|}
\hline 10 & $\begin{array}{l}\text { The American } \\
\text { journal of } \\
\text { hospice \& } \\
\text { palliative care }\end{array}$ & $\begin{array}{l}\text { Middle East } \\
\text { experience } \\
\text { in palliative } \\
\text { care } \\
\text { by Zeinah, } \\
\text { Ghaith F } \\
\text { Abu; Al- } \\
\text { Kindi, } \\
\text { Sadeer G; } \\
\text { Hassan, Azza } \\
\text { Adel }\end{array}$ & $02 / 2013$ & International Review & International Review & $\begin{array}{l}\text { Internat } \\
\text { ional } \\
\text { Review }\end{array}$ & 30 & 94 & $\begin{array}{l}\text { United } \\
\text { States }\end{array}$ \\
\hline 11 & $\begin{array}{l}\text { International } \\
\text { Journal of } \\
\text { Palliative } \\
\text { Nursing }\end{array}$ & $\begin{array}{l}\text { Knowledge } \\
\text { of and } \\
\text { attitudes } \\
\text { towards } \\
\text { palliative } \\
\text { care among } \\
\text { multinationa } \\
\text { I nurses in } \\
\text { Saudi Arabia } \\
\text { by Abudari, } \\
\text { Gassan; } \\
\text { Zahreddine, } \\
\text { Hassan; } \\
\text { Hazeim, } \\
\text { Hassan; Assi, } \\
\text { Mohammad } \\
\text { Al; Emara, } \\
\text { Sania }\end{array}$ & $09 / 2014$ & $\begin{array}{l}\text { Results The nurses scored } \\
\text { a mean of } 111.66 \text { out of } \\
150 \text { on the FATCOD scale } \\
\text { and of } 9.06 \text { out of } 20 \text { on } \\
\text { the PCQN. These scores } \\
\text { indicate moderate } \\
\text { attitudes towards but a } \\
\text { knowledge deficit } \\
\text { regarding palliative care. } \\
\text { The nurses' palliative care } \\
\text { training and years of } \\
\text { nursing experience } \\
\text { significantly affected the } \\
\text { scores. The level of } \\
\text { palliative care integration } \\
\text { in the nurses' home } \\
\text { countries was the most } \\
\text { significant factor in } \\
\text { multiple regression tests. }\end{array}$ & $\begin{array}{l}\text { Method A questionnaire } \\
\text { including demographic } \\
\text { data, the Palliative Care } \\
\text { Quiz for Nurses (PCQN), } \\
\text { and Frommelt Attitude } \\
\text { Toward Care of the } \\
\text { Dying scale (FATCOD) }\end{array}$ & $\begin{array}{l}395 \\
\text { staff } \\
\text { nurses } \\
\text { from } 19 \\
\text { countrie } \\
\text { s }\end{array}$ & 20 & 9 & $\begin{array}{l}\text { United } \\
\text { Kingdo } \\
\mathrm{m}\end{array}$ \\
\hline
\end{tabular}




\begin{tabular}{|c|c|c|c|c|c|c|c|c|c|}
\hline 12 & $\begin{array}{l}\text { Indian Journal } \\
\text { of palliative } \\
\text { care }\end{array}$ & $\begin{array}{l}\text { Pattern of } \\
\text { referral of } \\
\text { noncancer } \\
\text { patients to } \\
\text { palliative } \\
\text { care in the } \\
\text { eastern } \\
\text { province of } \\
\text { Saudi Arabia } \\
\text { by Ghanem, } \\
\text { Hafez M; } \\
\text { Shaikh, } \\
\text { Rawabi M; } \\
\text { Alia, Ahmad } \\
\text { M Abou; Al- } \\
\text { Zayir, Amani } \\
\text { S; Alsirafy, } \\
\text { Samy A }\end{array}$ & $09 / 2011$ & $\begin{array}{l}\text { From } 474 \text { patients, } 20 \\
(4.2 \%) \text { had a noncancer } \\
\text { diagnosis. The main } \\
\text { reason for the referral of } \\
\text { noncancer patients was } \\
\text { pain control. The most } \\
\text { prevalent diagnoses were } \\
\text { sickle cell disease (SCD) in } \\
6(30 \%) \text { patients and } \\
\text { peripheral arterial disease } \\
\text { (PAD) in } 5 \text { ( } 25 \%\end{array}$ & $\begin{array}{l}\text { This is a retrospective } \\
\text { review of the "palliative } \\
\text { care inpatient database" } \\
\text { of } 21 \text { months. }\end{array}$ & $x$ & 17 & $\begin{array}{l}235 \\
- \\
237\end{array}$ & India \\
\hline 13 & $\begin{array}{l}\text { Palliative \& } \\
\text { supportive } \\
\text { care }\end{array}$ & $\begin{array}{l}\text { The need for } \\
\text { more and } \\
\text { better } \\
\text { palliative } \\
\text { care for } \\
\text { Muslim } \\
\text { patients } \\
\text { by Harford, } \\
\text { Joe B; } \\
\text { Aljawi, } \\
\text { Deena M }\end{array}$ & $02 / 2013$ & From the Guest Editors & From the Guest Editors & $\begin{array}{l}\text { From } \\
\text { the } \\
\text { Guest } \\
\text { Editors }\end{array}$ & 11 & 1 & $\begin{array}{l}\text { United } \\
\text { Kingdo } \\
\mathrm{m}\end{array}$ \\
\hline
\end{tabular}




\begin{tabular}{|c|c|c|c|c|c|c|c|c|c|}
\hline 14 & $\begin{array}{l}\text { American } \\
\text { Journal of } \\
\text { Hospice and } \\
\text { Palliative } \\
\text { Medicine }\end{array}$ & $\begin{array}{l}\text { The Pattern } \\
\text { of } \\
\text { Antimicrobia } \\
\text { I Use for } \\
\text { Palliative } \\
\text { Care In- } \\
\text { Patients } \\
\text { During the } \\
\text { Last Week of } \\
\text { Life } \\
\text { by Abduh Al- } \\
\text { Shaqi, } \\
\text { Mohammed; } \\
\text { Alami, } \\
\text { Ahmad } \\
\text { Hasan; } \\
\text { Zahrani, Ali } \\
\text { Saeed Al; Al- } \\
\text { Marshad, } \\
\text { Batlah; } \\
\text { Muammar, } \\
\text { Abdulrahma } \\
\text { n Bin; MZ, } \\
\text { Al-Shahri }\end{array}$ & $02 / 2012$ & $\begin{array}{l}\text { RESULTS: } \\
\text { Of } 138 \text { patients who died } \\
\text { with advanced cancer in } \\
\text { the TPCU, } 87 \text { (63\%) were } \\
\text { on one or more } \\
\text { antimicrobials during their } \\
\text { last week of life. } \\
\text { Antibiotics were more } \\
\text { frequently used as } \\
\text { compared to antifungal } \\
\text { and antiviral agents, } 64 \\
\text { ( } 46.4 \%) ; 45 \text { ( } 32.6 \%) ; \text { and } 2 \\
\text { (1.5\%), respectively. About } \\
\text { one-third ( } 31.3 \%) \text { of } \\
\text { patients who received } \\
\text { antibiotics during their last } \\
\text { week of life were } \\
\text { prescribed more than one } \\
\text { antibiotic. Antimicrobials } \\
\text { were mostly given } \\
\text { systemically ( } 79 \% \text { ) rather } \\
\text { than topically ( } 21 \% \text { ). The } \\
\text { most common rationales } \\
\text { for antimicrobial } \\
\text { prescribing were oral } \\
\text { thrush in } 36 \text { patients } \\
\text { ( } 25.4 \% \text { ), wound care in } 29 \\
\text { patients ( } 20.4 \% \text { ), and on } \\
\text { empirical basis in } 29 \\
\text { patients ( } 20.4 \%) \text {. }\end{array}$ & $\begin{array}{l}\text { METHODS: } \\
\text { Medical records of all } \\
\text { patients who died in the } \\
\text { TPCU over a 14-month } \\
\text { period were reviewed } \\
\text { for demographics as well } \\
\text { as the frequency and } \\
\text { rationale of } \\
\text { antimicrobial use during } \\
\text { the patients' last week of } \\
\text { life. Information on } \\
\text { antimicrobial use was } \\
\text { obtained from a } \\
\text { computerized pharmacy } \\
\text { database. }\end{array}$ & $\begin{array}{l}138 \\
\text { patients } \\
: 60 \\
\text { male, } 78 \\
\text { female }\end{array}$ & 29 & 60 & \\
\hline 15 & $\begin{array}{l}\text { American } \\
\text { Journal of }\end{array}$ & $\begin{array}{l}\text { Legal Issues } \\
\text { in End of life }\end{array}$ & $04 / 2009$ & literature review & literature review & $\begin{array}{l}\text { literatur } \\
\text { e review }\end{array}$ & 26 & 119 & $\begin{array}{l}\text { United } \\
\text { States }\end{array}$ \\
\hline
\end{tabular}




\begin{tabular}{|c|c|c|c|c|c|c|c|c|c|}
\hline & $\begin{array}{l}\text { Hospice and } \\
\text { Palliative } \\
\text { Medicine }\end{array}$ & $\begin{array}{l}\text { Care: } \\
\text { Perspectives } \\
\text { from Saudi } \\
\text { Arabia and } \\
\text { the United } \\
\text { States } \\
\text { by Babgi, } \\
\text { Amani }\end{array}$ & & & & & & & \\
\hline 16 & $\begin{array}{l}\text { Middle East } \\
\text { Journal of } \\
\text { Cancer }\end{array}$ & $\begin{array}{l}\text { Saudi Cancer } \\
\text { Patients' } \\
\text { Attitudes } \\
\text { towards } \\
\text { Disclosure of } \\
\text { Cancer } \\
\text { Information } \\
\text { By Ali M. Al- } \\
\text { Amri }\end{array}$ & 2010 & $\begin{array}{l}\text { Results: The vast majority } \\
\text { of Saudi cancer patients } \\
\text { wanted to know the } \\
\text { diagnosis of cancer (98\%) } \\
\text { and only } 2 \% \text { wanted the } \\
\text { information to remain } \\
\text { undisclosed. Seventy } \\
\text { percent of the women } \\
\text { wanted family members } \\
\text { to know compared to only } \\
39 \% \text { of the men (P<0.001). } \\
\text { Only } 10 \% \text { of the patients } \\
\text { wanted their friends to } \\
\text { know. In this study, } 99 \% \\
\text { and } 98 \% \text {, respectively, } \\
\text { wanted to know about the } \\
\text { benefits of therapy and } \\
\text { about their diagnosis of } \\
\text { cancer. Of both genders, } \\
98 \% \text { also wanted to know } \\
\text { the side effects of therapy } \\
\text { and the prognosis. The } \\
\text { attitudes of Saudi men and } \\
\text { women with cancer were }\end{array}$ & $\begin{array}{l}\text { Methods: In this cross- } \\
\text { sectional questionnaire- } \\
\text { based prospective } \\
\text { survey, we interviewed } \\
332 \text { Saudi cancer } \\
\text { patients who received } \\
\text { oncological care at King } \\
\text { Fahd University Hospital, } \\
\text { Al-Khobar, Saudi Arabia } \\
\text { from July } 2002 \text { to July } \\
2009 \text { to explore their } \\
\text { attitudes regarding } \\
\text { disclosure of cancer } \\
\text { information. }\end{array}$ & 332 & 1 & $\begin{array}{l}175 \\
- \\
180\end{array}$ & Iran \\
\hline
\end{tabular}




\begin{tabular}{|c|c|c|c|c|c|c|c|c|c|}
\hline & & & & $\begin{array}{l}\text { almost identical apart } \\
\text { from sharing information } \\
\text { with their family } \\
\text { members. } 99 \% \text { of eastern } \\
\text { region cancer patients } \\
\text { wanted the diagnosis of } \\
\text { cancer disclosed } \\
\text { compared to } 74 \% \text { of those } \\
\text { from other regions } \\
(P=0.04) \text {. }\end{array}$ & & & & & \\
\hline 17 & $\begin{array}{l}\text { Journal of pain } \\
\text { \& palliative } \\
\text { care } \\
\text { pharmacother } \\
\text { apy }\end{array}$ & $\begin{array}{l}\text { Palliative } \\
\text { care in Saudi } \\
\text { Arabia: a } \\
\text { brief history } \\
\text { by Nixon, } \\
\text { Alan }\end{array}$ & 2003 & Original Article & Original Article & $\begin{array}{l}\text { Original } \\
\text { Article }\end{array}$ & 17 & 45 & $\begin{array}{l}\text { United } \\
\text { States }\end{array}$ \\
\hline 18 & $\begin{array}{l}\text { Asian Pacific } \\
\text { Journal of } \\
\text { Cancer } \\
\text { Prevention }\end{array}$ & $\begin{array}{l}\text { Pain coping } \\
\text { behaviours } \\
\text { of Saudi } \\
\text { patients } \\
\text { suffering } \\
\text { from } \\
\text { advanced } \\
\text { cancer: a } \\
\text { revisited } \\
\text { experience. } \\
\text { By Babgi } \\
\text { AA1. }\end{array}$ & 2010 & $\begin{array}{l}\text { RESULTS: Patients age } \\
\text { were almost equally } \\
\text { distributed among thirties } \\
(22 \%) \text {, forties ( } 24.2 \%) \text {, } \\
\text { fifties }(20.5 \%) \text {, and sixties } \\
(22.7 \%) \text {. Females ( } 59.1 \%) \\
\text { were slightly more than } \\
\text { males ( } 40.9 \%) \text {. About one- } \\
\text { half ( } 47 \%) \text { of them were } \\
\text { diagnosed since one--two } \\
\text { years age and slightly } \\
\text { more than half ( } 56.8 \%) \text { of } \\
\text { them were unaware of } \\
\text { their diagnosis. The major } \\
\text { adaptive and Active pain }\end{array}$ & $\begin{array}{l}\text { MATERIALS AND } \\
\text { METHODS: A descriptive } \\
\text { design was used for this } \\
\text { study to assess the pain } \\
\text { coping behavior Among } \\
\text { Saudi patients suffering } \\
\text { from advanced cancer. } \\
\text { The study was } \\
\text { conducted at the two } \\
\text { tumor centres which } \\
\text { deal with cancer patients } \\
\text { in Jeddah City. A } \\
\text { convenient sample of } \\
\text { 132 patients with } \\
\text { advanced cancer who }\end{array}$ & 132 & 11 & $\begin{array}{l}103 \\
- \\
106\end{array}$ & Korea \\
\hline
\end{tabular}




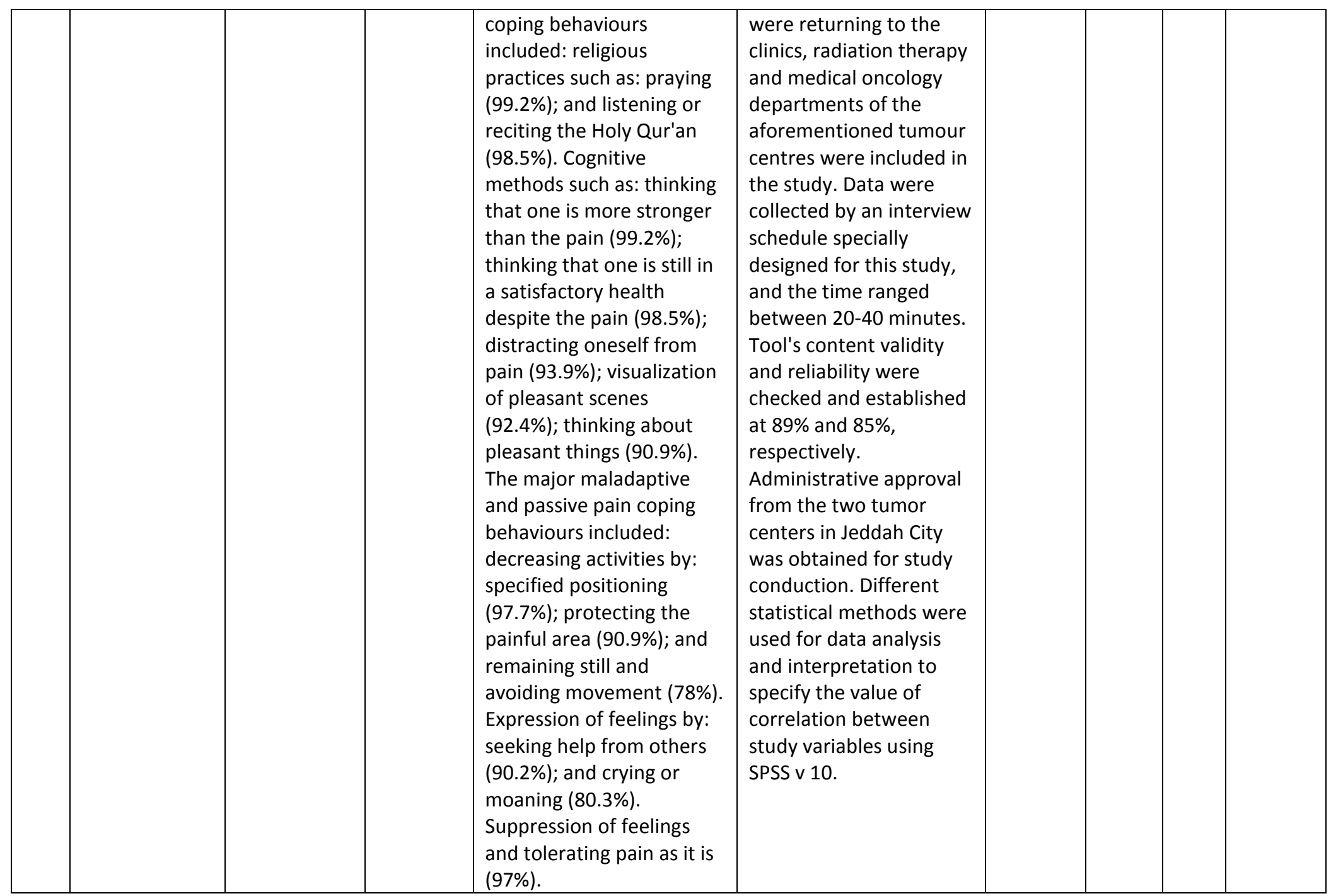




\begin{tabular}{|c|c|c|c|c|c|c|c|c|c|}
\hline 19 & $\begin{array}{l}\text { Journal of } \\
\text { Health } \\
\text { Specialties }\end{array}$ & $\begin{array}{l}\text { Exploring } \\
\text { the } \\
\text { perspectives } \\
\text { of nurses, } \\
\text { physicians, } \\
\text { and } \\
\text { healthcare } \\
\text { administrato } \\
\text { rs in Saudi } \\
\text { Arabian } \\
\text { hospitals on } \\
\text { palliative } \\
\text { care and } \\
\text { palliative } \\
\text { care nursing } \\
\text { By } \\
\text { Almobarak, } \\
\text { Fhaied }\end{array}$ & 2014 & Short review article & Short review article & \begin{tabular}{|l|} 
Short \\
review \\
article
\end{tabular} & 2 & $\begin{array}{l}54- \\
58\end{array}$ & $\begin{array}{l}\text { Saudi } \\
\text { Arabia }\end{array}$ \\
\hline 20 & $\begin{array}{l}\text { Journal of } \\
\text { Health } \\
\text { Specialties }\end{array}$ & $\begin{array}{l}\text { Palliative } \\
\text { care in Saudi } \\
\text { Arabia: Two } \\
\text { decades of } \\
\text { progress and } \\
\text { going strong } \\
\text { By } \\
\text { Sami } \\
\text { AlshammAls } \\
\text { uhail } \\
\text { Abdullah } \\
\text { Balaji P } \\
\text { Duraisam } \\
\text { Mahmoud } \\
\text { Anbar }\end{array}$ & 2014 & Short review article & Short review article & $\begin{array}{l}\text { Short } \\
\text { review } \\
\text { article }\end{array}$ & 2 & $\begin{array}{l}59- \\
60\end{array}$ & $\begin{array}{l}\text { Saudi } \\
\text { Arabia }\end{array}$ \\
\hline
\end{tabular}




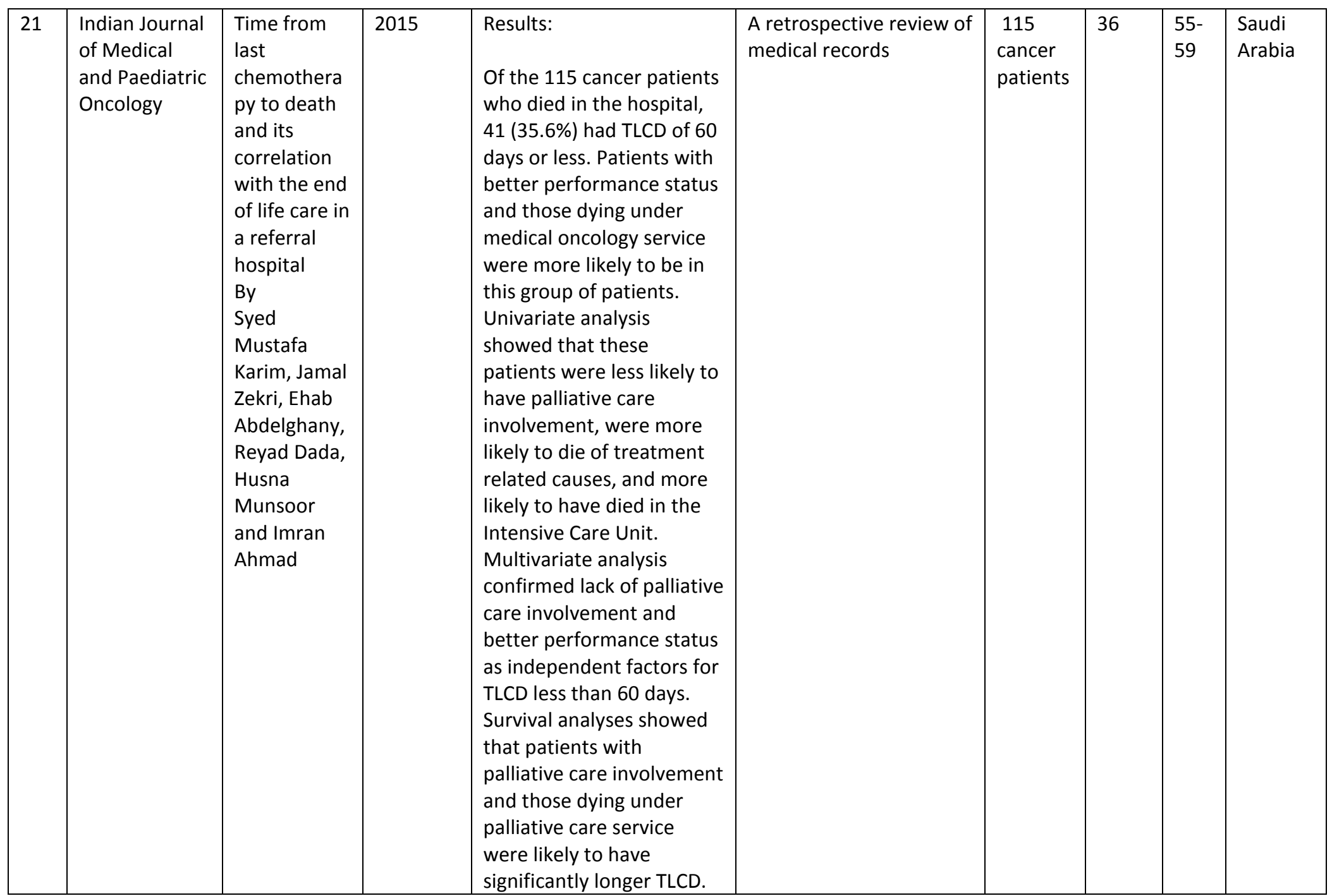




\begin{tabular}{|c|c|c|c|c|c|c|c|c|c|}
\hline 22 & $\begin{array}{l}\text { European } \\
\text { Journal of } \\
\text { Academic } \\
\text { Essays }\end{array}$ & $\begin{array}{l}\text { Prioritizing } \\
\text { Palliative } \\
\text { Care: Assess } \\
\text { Undergradu } \\
\text { ate Nursing } \\
\text { Curriculum, } \\
\text { knowledge } \\
\text { and Attitude } \\
\text { among } \\
\text { Nurses } \\
\text { Caring End } \\
\text { of life } \\
\text { Patients } \\
\text { By } \\
\text { Youssef H. A. } \\
\text { M., Mansour } \\
\text { M. A.M., Al- } \\
\text { Zahrani S. S. } \\
\text { M., Ayasreh } \\
\text { I. R. A. and } \\
\text { Abd El- } \\
\text { Karim R. A. } \\
\text { K. }\end{array}$ & 2015 & $\begin{array}{l}\text { Results: Above half of } \\
\text { nurses had poor } \\
\text { knowledge regarding } \\
\text { palliative care, but most of } \\
\text { them showed positive } \\
\text { attitude regarding end of } \\
\text { life care especially above } \\
\text { diploma degree holders. } \\
\text { While most of the content } \\
\text { of End of life (EOL) care in } \\
\text { nursing educational } \\
\text { program was perceived by } \\
\text { faculty members as } \\
\text { inadequate and they } \\
\text { agreed on the importance } \\
\text { of EOL as a part of nursing } \\
\text { curriculum. They also } \\
\text { perceived that the } \\
\text { greatest barriers for } \\
\text { improving EOL care were } \\
\text { "Inadequate content", "No } \\
\text { special training for end of } \\
\text { life", and "No special } \\
\text { governmental hospital" }\end{array}$ & $\begin{array}{l}\text { A cross sectional } \\
\text { quantitative survey } \\
\text { study }\end{array}$ & $\begin{array}{l}100 \\
\text { nurses }\end{array}$ & 2 & $\begin{array}{l}90- \\
101\end{array}$ & Portugal \\
\hline 23 & $\begin{array}{l}\text { Middle East } \\
\text { Journal of } \\
\text { Nursing }\end{array}$ & $\begin{array}{l}\text { Nurses' } \\
\text { Knowledge } \\
\text { about } \\
\text { Palliative } \\
\text { Care in an } \\
\text { Intensive } \\
\text { Care Unit in } \\
\text { Saudi Arabia }\end{array}$ & 2015 & $\begin{array}{l}\text { Results: The palliative care } \\
\text { concept was not familiar } \\
\text { for most ICU nurses but it } \\
\text { was applied in their daily } \\
\text { work. Most nurses } \\
\text { provided physical care at } \\
\text { the end of life to keep the } \\
\text { body intact. Some nurses } \\
\text { highlighted that dying }\end{array}$ & $\begin{array}{l}\text { Method: Eight individual } \\
\text { qualitative } \\
\text { semistructured } \\
\text { interviews were } \\
\text { conducted. Interviews } \\
\text { were audiotaped and } \\
\text { transcribed verbatim. } \\
\text { Manifest content } \\
\text { analysis was used to }\end{array}$ & 8 & 9 & $\begin{array}{l}7- \\
13\end{array}$ & $\begin{array}{l}\text { Lebano } \\
\mathrm{n}\end{array}$ \\
\hline
\end{tabular}




\begin{tabular}{|c|c|c|c|c|c|c|c|c|}
\hline & & $\begin{array}{l}\text { Zahra } \\
\text { Alshaikh, } \\
\text { Mohammed } \\
\text { Alkhodari, } \\
\text { Taina } \\
\text { Sormunen,P } \\
\text { ernilla } \\
\text { Hillerås }\end{array}$ & & $\begin{array}{l}\text { patients did not feel pain } \\
\text { to be treated and did not } \\
\text { have emotions to be } \\
\text { supported. }\end{array}$ & $\begin{array}{l}\text { analyze the data. } \\
\text { Results: }\end{array}$ & & & \\
\hline 24 & $\begin{array}{l}\text { International } \\
\text { Journal of } \\
\text { Research in } \\
\text { Nursing }\end{array}$ & $\begin{array}{l}\text { The } \\
\text { Experience } \\
\text { Of Overseas } \\
\text { Nurses } \\
\text { Caring For } \\
\text { Muslim } \\
\text { Patients In } \\
\text { Kingdom Of } \\
\text { Saudi Arabia } \\
\text { And Uae: A } \\
\text { Qualitative } \\
\text { Study } \\
\text { By } \\
\text { Sami } \\
\text { AlYateem } \\
\text { and Nabeel } \\
\text { Al-Yateem }\end{array}$ & 2014 & $\begin{array}{l}\text { The study revealed } \\
\text { three themes and five } \\
\text { subthemes. The main } \\
\text { themes were: (1) Right } \\
\text { to practice Vs impact on } \\
\text { care: A matter of } \\
\text { balance; (2) Islamic } \\
\text { principles: Nursesâ } \square \text { TM } \\
\text { knowledge; and (3) } \\
\text { Broken lines of contact } \\
\text { with social systems and } \\
\text { patients. Most of } \\
\text { participants have } \\
\text { demonstrated } \\
\text { insufficient knowledge } \\
\text { and experience of } \\
\text { Islamic principles which } \\
\text { cannot aid them in } \\
\text { providing culturally } \\
\text { competent nursing care. } \\
\text { It has also revealed that } \\
\text { nurses should further } \\
\text { recognize religion as an } \\
\text { important part of the } \\
\text { healing process and not } \\
\text { as a factor that } \\
\text { negatively impacts their }\end{array}$ & $\begin{array}{l}\text { A qualitative, } \\
\text { exploratory approach } \\
\text { utilizing participants' } \\
\text { written narratives was } \\
\text { adopted within this } \\
\text { research study. }\end{array}$ & $\begin{array}{l}31 \\
\text { nurses }\end{array}$ & 5 & $\begin{array}{l}17- \\
26\end{array}$ \\
\hline
\end{tabular}




\begin{tabular}{|c|c|c|c|c|c|c|c|c|c|}
\hline & & & & $\begin{array}{l}\text { role and hinders patient } \\
\text { care. }\end{array}$ & & & & & \\
\hline 25 & $\begin{array}{l}\text { Gulf Journal of } \\
\text { Oncology }\end{array}$ & $\begin{array}{l}\text { Appropriate } \\
\text { ness of } \\
\text { cancer pain } \\
\text { manageme } \\
\text { nt in Saudi } \\
\text { teaching } \\
\text { hospital. } \\
\text { Al-Rowaili } \\
\text { A1, Al- } \\
\text { aqeel SA, } \\
\text { Al-Naim } \\
\text { LS, Al-Diab } \\
\text { Al. }\end{array}$ & 2009 & $\begin{array}{l}80(50 \%) \text { reported } \\
\text { moderate or severe pain. } \\
40 \% \text { of those with pain } \\
\text { were not given any } \\
\text { pain medication. Sixty } \\
\text { percent of the patients } \\
\text { had appropriate pain } \\
\text { management. Pain } \\
\text { documentation was } \\
\text { inappropriate and needs } \\
\text { improvement in } 57 \% \text { of } \\
\text { the patients. There } \\
\text { was under utilization of } \\
\text { NSAIDs which were } \\
\text { prescribed in } 8(5 \%) \\
\text { patients only from those } \\
\text { on pain medication. } \\
\text { Transdermal fentanyl was } \\
\text { the most frequently used } \\
\text { opioid (21\%) for } \\
\text { moderate to severe pain. } \\
\text { Therefore, despite } \\
\text { published guidelines for } \\
\text { pain management, many } \\
\text { patients with cancer } \\
\text { receive inadequate } \\
\text { analgesia. }\end{array}$ & $\begin{array}{l}\text { A prospective } \\
\text { observational study }\end{array}$ & $\begin{array}{l}160 \\
\text { patients }\end{array}$ & 5 & $\begin{array}{l}37- \\
43\end{array}$ & Kuwait \\
\hline
\end{tabular}




\begin{tabular}{|c|c|c|c|c|c|c|c|c|c|}
\hline 26 & $\begin{array}{l}\text { Qatar Medical } \\
\text { Journal }\end{array}$ & $\begin{array}{l}\text { Prevalence } \\
\text { and severity } \\
\text { of pain in } \\
\text { cancer } \\
\text { patients in } \\
\text { an outpatient } \\
\text { palliative } \\
\text { care setting } \\
\text { in Saudi } \\
\text { Arabia } \\
\text { By } \\
\text { Omar Al- } \\
\text { Zahrani, } \\
\text { Abdelmonei } \\
\text { m Eldali, } \\
\text { And } \\
\text { Mohammad } \\
\text { Zafir Al- } \\
\text { Shahri }\end{array}$ & 2014 & $\begin{array}{l}\text { Results: A total of } 124 \\
\text { patients were interviewed, } \\
\text { of whom } 73 \text { ( } 59 \% \text { ) were } \\
\text { females. The majority of } \\
\text { patients ( } 82.3 \% \text { ) had } \\
\text { metastatic disease. The } \\
\text { most common cancers } \\
\text { were breast ( } 27.4 \% \text { ) and } \\
\text { head and neck (15.3\%). } \\
\text { The majority of patients } \\
\text { (85.5\%) reported pain, } \\
\text { with a median intensity } \\
\text { score of } 5 \text { and a mean of } \\
4.6 . \text { Of those who } \\
\text { reported pain, } 54 \text { ( } 51 \%) \\
\text { scored above } 4 . \text { The mean } \\
\text { pain score did not differ } \\
\text { between groups of } \\
\text { patients according to } \\
\text { various characteristics } \\
\text { such as age, sex, } \\
\text { performance status, type } \\
\text { of cancer or encounter } \\
\text { type. }\end{array}$ & $\begin{array}{l}\text { Methods: In a PC } \\
\text { outpatient setting in a } \\
\text { major tertiary hospital in } \\
\text { SA, we interviewed adult } \\
\text { patients with cancer } \\
\text { during their attendance } \\
\text { to the clinic. Patients } \\
\text { were requested to score } \\
\text { the severity of their pain } \\
\text { on a } 0 \text { to } 10 \text { numerical } \\
\text { scale. }\end{array}$ & $\begin{array}{l}124 \\
\text { patients }\end{array}$ & 1 & $\begin{array}{l}38- \\
45\end{array}$ & Qatar \\
\hline 27 & $\begin{array}{l}\text { Palliative } \\
\text { Medicine }\end{array}$ & $\begin{array}{l}\text { Implementat } \\
\text { ion of the } \\
\text { World } \\
\text { Health } \\
\text { Organization } \\
\text { 'analgesic } \\
\text { ladder' in } \\
\text { Saudi } \\
\text { Arabia. }\end{array}$ & 2001 & $\begin{array}{l}\text { General } \\
\text { Availability of medication } \\
\text { Access to palliative } \\
\text { (bereavement) services } \\
\text { Education } \\
\text { Poor education re } \\
\text { palliative care - all } \\
\text { healthcare workers }\end{array}$ & $\begin{array}{l}\text { Materials and methods } \\
\text { Approximately } 150 \\
\text { participants attended } \\
\text { the workshop, } \\
\text { with roughly equal } \\
\text { numbers of males and } \\
\text { females. The majority of } \\
\text { participants came from } \\
\text { the }\end{array}$ & $\begin{array}{l}150 \\
\text { particip } \\
\text { ants }\end{array}$ & 15 & $\begin{array}{l}135 \\
- \\
145\end{array}$ & $\begin{array}{l}\text { United } \\
\text { Kingdo } \\
\mathrm{m}\end{array}$ \\
\hline
\end{tabular}




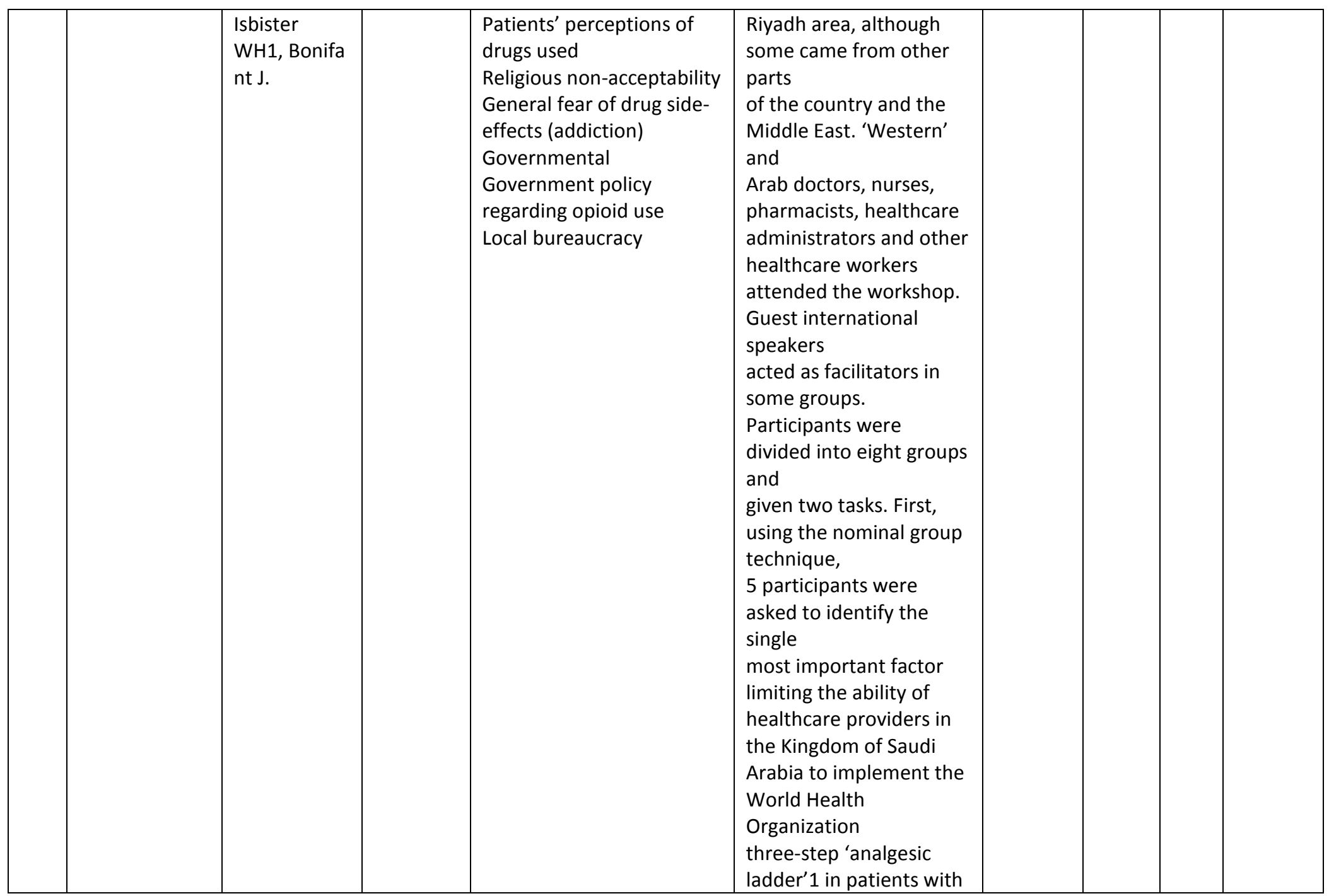




\begin{tabular}{|c|c|c|c|c|c|c|c|c|c|}
\hline & & & & & $\begin{array}{l}\text { pain due to advanced } \\
\text { cancer. Secondly, } \\
\text { participants } \\
\text { were instructed to } \\
\text { suggest solutions to the } \\
\text { problem } \\
\text { that they had identified. } \\
\text { Each group had a } \\
\text { chairman } \\
\text { and a reporter: the latter } \\
\text { presented the group's } \\
\text { deliberations to a } \\
\text { plenary session of all } \\
\text { participants. }\end{array}$ & & & & \\
\hline 28 & $\begin{array}{l}\text { Journal of } \\
\text { Oncology } \\
\text { Pharmacy } \\
\text { Practice }\end{array}$ & $\begin{array}{l}\text { Insights } \\
\text { about } \\
\text { health- } \\
\text { related } \\
\text { quality of } \\
\text { life in cancer } \\
\text { patients } \\
\text { indicate } \\
\text { demands for } \\
\text { better } \\
\text { pharmaceuti } \\
\text { cal care. } \\
\text { By } \\
\text { Ibrahim } \\
\mathrm{NA}, \text { Björnsd } \\
\text { ottir I, Al } \\
\text { Alwan } \\
\text { AS, Honore } \\
\text { PH. }\end{array}$ & 2013 & $\begin{array}{l}\text { RESULTS: } \\
\text { Quality of life was } \\
\text { evaluated for } 87 \\
\text { participants. Most of } \\
\text { patients were aged } \\
\text { between } 51 \text { and } 60 \text { years; } \\
\text { and } 50 \% \text { had active } \\
\text { treatment with } \\
\text { chemotherapy. Patients } \\
\text { seemed to perform well } \\
\text { with respect to average } \\
\text { scores in both the } \\
\text { symptoms and the } \\
\text { functional health status } \\
\text { scales. The mean score for } \\
\text { the global quality of life } \\
\text { scale was } 47.2 \pm 27.1 \text {, } \\
\text { while the range of mean } \\
\text { scores for the five function }\end{array}$ & $\begin{array}{l}\text { A cross-sectional } \\
\text { descriptive study }\end{array}$ & $\begin{array}{l}87 \\
\text { particip } \\
\text { ants }\end{array}$ & 20 & $\begin{array}{l}270 \\
- \\
277\end{array}$ & USA \\
\hline
\end{tabular}




\begin{tabular}{|c|c|c|c|c|c|c|c|c|c|}
\hline & & & & $\begin{array}{l}\text { subscales was } 59.0 \pm 27.1 \\
\text { to } 81.6 \pm 13.8 \text {, indicating } \\
\text { average level of general } \\
\text { wellbeing with above } \\
\text { average to high level of } \\
\text { functional health status, } \\
\text { while }>50 \% \text { of the patients } \\
\text { met the operational } \\
\text { criterion having less } \\
\text { severe symptoms. } \\
\text { Outpatients generally had } \\
\text { somewhat higher scores } \\
\text { as compared to } \\
\text { hospitalized patients. }\end{array}$ & & & & & \\
\hline 29 & $\begin{array}{l}\text { Journal of } \\
\text { Family and } \\
\text { Community } \\
\text { Medicine }\end{array}$ & $\begin{array}{l}\text { Knowledge } \\
\text { of the } \\
\text { residents at } \\
\text { King Abdul- } \\
\text { Aziz } \\
\text { University } \\
\text { Hospital } \\
\text { (KAAUH) } \\
\text { about } \\
\text { palliative } \\
\text { care } \\
\text { By } \\
\text { Sultan H } \\
\text { Alamri }\end{array}$ & 2012 & $\begin{array}{l}\text { Results: Of the } 80 \\
\text { residents } 65 \text { (81\%) } \\
\text { responded, the } \\
\text { overwhelming majority } \\
\text { of whom were Saudis } \\
(92.3 \%) \text { with an equal } \\
\text { representation of males } \\
\text { and females. The mean } \\
\text { age of the participants } \\
\text { was } 29.1 \pm 2.4 \text { years. } \\
\text { Less than one-third } \\
(29.2 \%) \text { indicated that } \\
\text { they had previous } \\
\text { didactic education on } \\
\text { palliative care. The } \\
\text { percentage of right } \\
\text { answers on items } \\
\text { reflecting knowledge on } \\
\text { palliative care } \\
\end{array}$ & $\begin{array}{l}\text { a cross-sectional } \\
\text { design }\end{array}$ & $\begin{array}{l}80 \\
\text { residen } \\
\text { ts }\end{array}$ & 19 & $\begin{array}{l}194 \\
- \\
197\end{array}$ & $\begin{array}{l}\text { Saudi } \\
\text { Arabia }\end{array}$ \\
\hline
\end{tabular}




\begin{tabular}{|c|c|c|c|c|c|c|c|c|c|}
\hline & & & & $\begin{array}{l}\text { accounted for } 29.9 \% \pm \\
9.9 \% \text {. No statistically } \\
\text { significant difference } \\
\text { was found in the level of } \\
\text { knowledge among the } \\
\text { residents according to } \\
\text { their demographics or } \\
\text { graduation and training } \\
\text { characteristics. }\end{array}$ & & & & & \\
\hline 30 & $\begin{array}{l}\text { Intensive Care } \\
\text { Medicine }\end{array}$ & $\begin{array}{l}\text { Compliance } \\
\text { with DNR } \\
\text { policy in a } \\
\text { tertiary care } \\
\text { center in } \\
\text { Saudi } \\
\text { Arabia. } \\
\text { Gouda A, Al- } \\
\text { Jabbary } \\
\text { A, Fong L. }\end{array}$ & 2010 & $\begin{array}{l}\text { DNR was initiated in } 65 \\
\text { patients referred to the } \\
\text { intensive care unit (ICU): } \\
46.2 \% \text { females, } 53.8 \% \\
\text { males; age range } 19-93 \\
\text { years, mean } \pm \text { standard } \\
\text { deviation (SD) } 66.1 \pm 16.0 \\
\text { years. DNR was initiated } \\
\text { by ICU physician in } 80 \% \text { of } \\
\text { cases and by most } \\
\text { responsible physician } \\
\text { (MRP) in } 20 \% \text { of cases. } \\
\text { There was a delay (of } \\
\text { more than } 48 \text { h) in } \\
\text { completing MRP signature } \\
\text { in } 8 \text { patients ( } 12.3 \% \text { ), and } \\
\text { no signature at all by the } \\
\text { MRP in } 13 \text { patients ( } 20 \% \text { ). } \\
\text { Documentation of } \\
\text { discussion with the family } \\
\text { was absent in } 53.8 \% \text { of } \\
\text { cases. }\end{array}$ & $\begin{array}{l}\text { A cohort study of data } \\
\text { prospectively collected }\end{array}$ & $\begin{array}{l}65 \\
\text { patients }\end{array}$ & 36 & $\begin{array}{l}214 \\
9- \\
53\end{array}$ & France \\
\hline
\end{tabular}




\begin{tabular}{|c|c|c|c|c|c|c|c|c|c|}
\hline 31 & \begin{tabular}{|l|} 
Journal of \\
Transcultural \\
Nursing
\end{tabular} & $\begin{array}{l}\text { Culturally } \\
\text { sensitive } \\
\text { caring for } \\
\text { Saudi } \\
\text { patients. } \\
\text { Al-Shahri } \\
\text { MZ. } \\
\end{array}$ & 2002 & Review article & Review article & $\begin{array}{l}\text { Review } \\
\text { article }\end{array}$ & 13 & $\begin{array}{l}133 \\
-8\end{array}$ & USA \\
\hline 32 & $\begin{array}{l}\text { Pediatrics. } \\
\text { Official journal } \\
\text { of the } \\
\text { American } \\
\text { Academy of } \\
\text { Pediatrics. }\end{array}$ & $\begin{array}{l}\text { A Saudi } \\
\text { Family } \\
\text { Making End } \\
\text { of life } \\
\text { Decisions in } \\
\text { the PICU } \\
\text { By } \\
\text { Sadeth } \\
\text { Sayeed, } \\
\text { Aasim } \\
\text { Padela, } \\
\text { Maryam Y. } \\
\text { Naim, John } \\
\text { D. Lantos }\end{array}$ & 2012 & Special Article & Special Article & $\begin{array}{l}\text { Special } \\
\text { Article }\end{array}$ & 129 & & USA \\
\hline 33 & $\begin{array}{l}\text { Annals of } \\
\text { Saudi } \\
\text { Medicine }\end{array}$ & $\begin{array}{l}\text { A "good } \\
\text { death": } \\
\text { perspectives } \\
\text { of Muslim } \\
\text { patients and } \\
\text { healthcare } \\
\text { providers } \\
\text { By } \\
\text { Mohamad A. } \\
\text { Tayeb, Ersan } \\
\text { Al-Zamel, } \\
\text { Muhammed }\end{array}$ & 2010 & $\begin{array}{l}\text { RESULT: On average, } \\
\text { each participant agreed on } \\
\text { eight principles of the } \\
\text { questionnaire. Dignity, } \\
\text { privacy, spiritual and } \\
\text { emotional support, access } \\
\text { to hospice care, ability to } \\
\text { issue advance directives, } \\
\text { and to have time to say } \\
\text { goodbye were the top } \\
\text { priorities. Participants } \\
\text { identified three main }\end{array}$ & $\begin{array}{l}\text { A 12-question } \\
\text { questionnaire based on } \\
\text { the } 12 \text { principles of the } \\
\text { TFHCOP good death } \\
\text { definition, followed by } \\
\text { face-to-face interviews. }\end{array}$ & $\begin{array}{l}284 \\
\text { Muslims } \\
\text { of both } \\
\text { genders }\end{array}$ & 30 & $\begin{array}{l}215 \\
- \\
221\end{array}$ & $\begin{array}{l}\text { Saudi } \\
\text { Arabia }\end{array}$ \\
\hline
\end{tabular}




\begin{tabular}{|c|c|c|c|c|c|c|c|c|c|}
\hline & & $\begin{array}{l}\text { M. Fareed, } \\
\text { and Hesham } \\
\text { A. Abouellail }\end{array}$ & & $\begin{array}{l}\text { domains. The first domain } \\
\text { was related to faith and } \\
\text { belief. The second domain } \\
\text { included some principles } \\
\text { related to self-esteem and } \\
\text { person>s image to friends } \\
\text { and family. The third } \\
\text { domain was related to } \\
\text { satisfaction about family } \\
\text { security after the death of } \\
\text { the patient. Professional } \\
\text { role distinctions were more } \\
\text { pronounced than were } \\
\text { gender or nationality } \\
\text { differences. }\end{array}$ & & & & & \\
\hline 34 & \begin{tabular}{|l|} 
RMIT \\
University
\end{tabular} & $\begin{array}{l}\text { Pain } \\
\text { Managemen } \\
\mathrm{t} \text { In Hail } \\
\text { Region } \\
\text { Hospitals In } \\
\text { Saudi Arabia } \\
\text { By } \\
\text { Hamdan } \\
\text { Albaqawi }\end{array}$ & 2014 & $\begin{array}{l}\text { In this study, the average } \\
\text { correct response rate to the } \\
\text { KASRP questionnaire was } \\
41.75 \% \text {, with rates } \\
\text { ranging from 5-87 \%. The } \\
\text { findings indicate } \\
\text { inadequate knowledge } \\
\text { regarding pain, pain } \\
\text { assessment, pain } \\
\text { management and pain } \\
\text { medications. When } \\
\text { analysed } \\
\text { using thematic analysis, } \\
\text { the qualitative data } \\
\text { highlight some barriers } \\
\text { that affect nurses' ability } \\
\text { to provide effective pain } \\
\text { management; these include }\end{array}$ & $\begin{array}{l}\text { an explorative, } \\
\text { descriptive, mixed- } \\
\text { methods (quantitative } \\
\text { and } \\
\text { qualitative) study }\end{array}$ & $\begin{array}{l}303 \\
\text { nurses } \\
\text { for } \\
\text { question } \\
\text { naire } \\
\text { and } \\
28 \\
\text { nurses } \\
\text { for } \\
\text { intervie } \\
\text { w }\end{array}$ & $\begin{array}{l}\text { Thesi } \\
\mathrm{s}\end{array}$ & $\begin{array}{l}\text { The } \\
\text { sis }\end{array}$ & $\begin{array}{l}\text { Australi } \\
a\end{array}$ \\
\hline
\end{tabular}




\begin{tabular}{|l|l|l|l|l|l|l|}
\hline & & & $\begin{array}{l}\text { language, workload, } \\
\text { inadequate numbers } \\
\text { of staff, lack of education } \\
\text { and cultural orientation } \\
\text { courses, and religious and } \\
\text { cultural factors. }\end{array}$ & & & \\
\hline
\end{tabular}


Appendix 2 Ethical Approval

MANCHESTER METROPOLITAN UNIVERSITY

FACULTY OF HEALTH, PSYCHOLOGY AND SOCIAL CARE

MEMORANDUM

FACULTY ACADEMIC ETHICS COMMITTEE

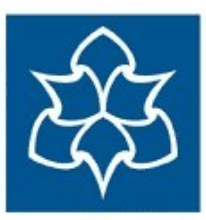

Manchester Metropolitan University

To: $\quad$ Fhaied Almobarak

From: Prof Carol Haigh

Date: $\quad 07 / 10 / 2013$

Subject: Ethics Application 1190

Title: Exploring the perspectives of nurses, physicians, and healthcare administrators in Saudi Arabian hospitals on palliative care and palliative care nursing

Thank you for your application for ethical approval.

The Faculty Academic Ethics Committee review process has recommended approval of your ethics application. This approval is granted for the duration of your research up to 42 months for full-time students or staff and 60 months for part-time students. Extensions to the approval period can be requested.

If your research changes you might need to seek ethical approval for the amendments. Please request an amendment form.

We wish you every success with your project. 
Prof Carol Haigh and Prof Jois Stansfield

Chair and Deputy Chair

Faculty Academic Ethics Committee 


\section{Appendix 3 Participant Information Sheet English}

Project Title: Exploring the perspectives of nurses, physicians, and healthcare administrators in Saudi Arabian hospitals on palliative care and palliative care nursing

Researcher: Fhaied Almobarak Lecturer in Palliative Care University of Dammam

Contact Number:

Effective patient care in hospitals is one of the most significant fields of concern in present-day medicine. One of the areas in this field is palliative care. In considering this area of patient care, one important aspect is to examine inputs from the perspective of healthcare professionals, specifically doctors, nurses, and hospital administrators. In line with this, we would like to invite you to participate in this study. This is a PhD project that has received ethical approval from the MMU. The following paragraphs explain details about the study and aspects in relation to your participation should you consent to it.

The purpose of this study is to determine the current state of palliative care in Saudi Arabia from the perspective of its healthcare professionals. Specifically, the research questions of the study are: (1) What are the perceptions of KSA hospital healthcare practitioners regarding palliative care and specialisation into palliative care? (2) What issues regarding the development of palliative care are evident in the KSA hospital setting? And (3) What recommendations can be made for strengthening the value of palliative care among healthcare practitioners in Saudi Arabia?

In line with this, we would like to find out about your views about palliative care and specializing in palliative care, along with your experiences in relation to the delivery of palliative care to patients in the hospital that you are working at through a oneon-one interview. This interview is expected to take between 20-30 minutes of your time, and may be audio recorded if you consent to such. The interview can be conducted at a place and time of your choosing, such as the hospital that you work at after your shift or in your home. However, the venue must be reasonably accessible to the interviewer. Also, in order to protect the interviewer should the venue be remote or isolated, the interviewer will inform his research supervisor about his whereabouts and the identity of the person who he will be interviewing.

In the interview, you will be asked to talk about your understanding of palliative care, about the extent to which palliative care is conducted at your hospital, and the extent to which your responsibilities entail the delivery of palliative care. Furthermore, you will be asked to discuss your perspectives about the importance of palliative care, 
and its relevance as a specialization both in general and in particular to your selected career path as a healthcare professional.

The interviews will be transcribed verbatim and analysed. A copy of the transcript will be provided to you so that you can review them and strike out or change any part that you deem appropriate to. Anonymous quotes from transcripts may appear in the results section of the final write-up.

Participation in this study is completely voluntary. Also, you may choose to withdraw your consent at any time in the study. No explanations on your part will be required. You may also refuse to answer any question asked, and you may have any part of the transcript of the interview stricken from the records of the research. There are minimal risks involved in participating in the study. We understand that some of the inputs that you may have to share can be controversial, and so we assure you that your anonymity as a participant in the study will be maintained at all times. You will not be personally identified in any of our records that relate directly to your transcript, and your contact information will be discarded after we deliver a copy of the interview transcript to you for verification.

There may be no direct benefits to the participants of the study but the results of this study can have applications that may lead to positive developments in patient care and the education of nurses, physicians and healthcare administrators.

We hope that you will consent to participating in this study. Attached to this information sheet is a consent form where you may indicate your decision. Thank you! 


\section{Appendix 4 Participant Information Sheet Arabic}

عنوان المشروع : استكثاف تصورات ومفاهيم الممرضين والأطباء ومديري الرعاية الصحية في المستشفيات السعودية حول الرعاية التلطيفية وتمريض الرعاية التلطيفية الباحث: فييد المبارك محاضر في الر عاية التلطيفية جامعة الدمام

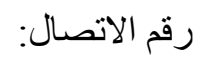

رعاية المرضى الفعالة في المستشفيات هي واحدة من المجالات الأكثر شأن وقلق وأهمية فيما يتعلق بشأن الطب

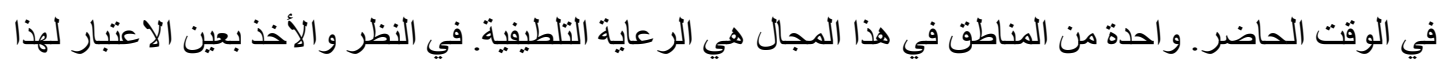

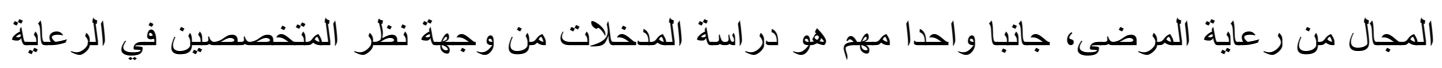

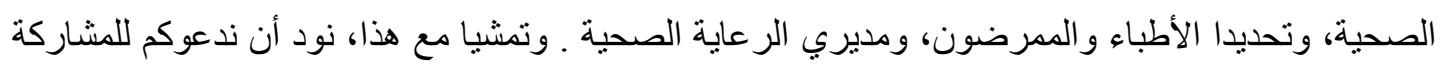

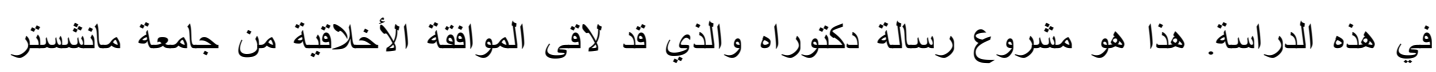
ميتروبولتين في المملكة المتحدة البريطانية. وتوضح الفقرات التالية تفاصيل عن الدراسة و الجو انب فيما بتعلق

$$
\text { بمشاركتكم و التي يجب أن تو افق عليها. }
$$

الغرض من هذه الدراسة هو تحديد الحالة الراهنة للرعاية التلطيفية في المملكة العربية السعودية من مفاهيم

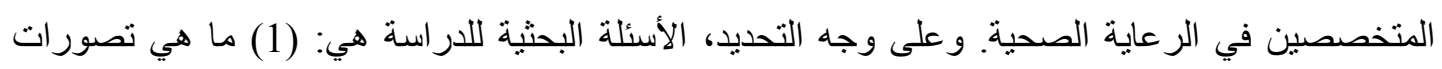

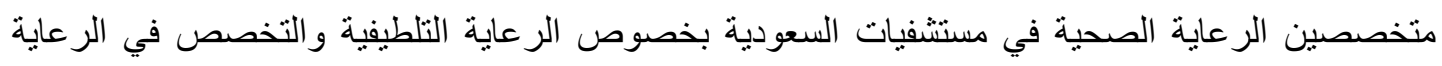
التلطيفية؟ (2) ما هي القضايا المتعلقة بتطوير الرعاية التلطيفية الواضحة و الظاهرة في المستثفيات السعودية؟ و (3) ما هي التوصيات التي يمكن أن تتم لتعزيز قيمة الرعاية التلطيفية بين متخصصين الرعاية الصحية في لئي المملكة العربية السعودية؟ مأبئ

و تمشيا مع هذا، نود أن نعرف مفاهيمكم حول الرعاية التلطيفية و التخصص في الر عاية التلطيفية، جنبا إلى جنب مع خبر اتكم فيما بتعلق بتقديم الرعاية التلطيفية للمرضى في المستشفى الذي تعمل فيه فئ من خلال مقابلة واحد

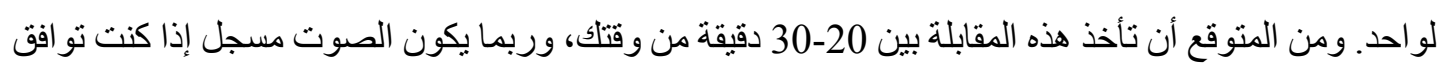
على هذا. ويمكن إجراء المقابلة في مكان ووقت من اختيارك، مثل المستشفى الذي تعمل فيه بعد الانتهاء من ون وند

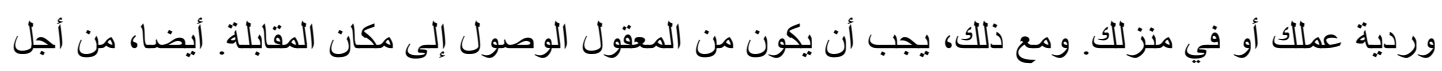

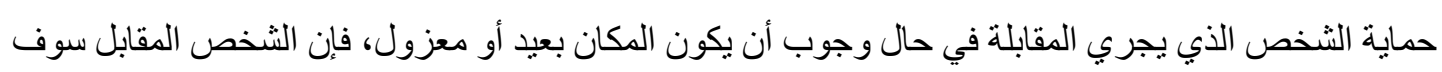
يبلغ مشرف بحثه عن مكان وجوده وهوية الثخص الذي سيتم إجر اء المقابلة معه.

في المقابلة، سوف يطلب منك أن تتحدث عن فهمك للر عاية التلطيفية، وحول المدى الذي تتم فيه الرعاية التلطيفية في المستشفى الذي تعمل به ، و إلى أي مدى مسؤولياتكم مترتبة على تقديم الرعاية التلطيفية. و علاوة على ذللك، 
سوف يطلب منك مناقثة وجهات نظرك حول أهمية الرعاية التلطيفية، وأهميتها كتخصص بصفة عامة وبصفة خاصة في مسارك الوظيفي الذي اخترته كمتخصص روعاية صحية.

سيتم تدوين المقابلات حرفيا وسيتم تحليلها ـ وسيتم تقديم نسخة للك من محضر المقابلة المدون بحيث يمكنك استعر اضه و شطب أو تغيير أي جزء ترونه مناسب لذلك ـ قد يظهر اقتباس مجهول من محضر المقابلة في قسم

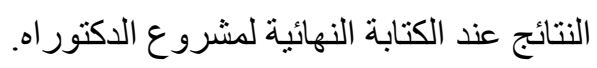

المشاركة في هذه الدر اسة هي طوعية و اختبارية تماما ـ أيضا ، قد تختار سحب مو افقتلك في أي وقت في الدر اسة. سوف لن يكون هناك حاجة الى شرح منك للسبب. قد ترفض أيضا الرد على أي سؤ ال ، و قد ترفض أي جزء أبهاء من نص المقابلة المكتشفة من سجلات البحث.

هناك حد أدنى من المخاطر التي ينطوي عليها المشاركة في الدراسة . نحن نفهم أن بعض المدخلات والتي قد تضطر إلى مشاركتها يمكن أن تكون مثيرة للجدل ، و هكذا نحن نضمن لكم أن هويتكم كمشارك في الدر اسة سيتم

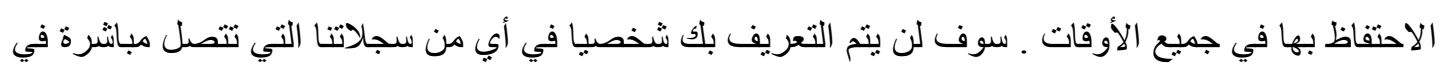
النص الخاص بك، و سوف يتم التخلص من معلومات الاتصال الخاصة بك بعد نسليم نسخة من محضر المقابلة للك للتحقق منها.

قد لا يكون هناك فو ائد مباثرة للمشاركين في الدر اسة ولكن نتائج هذه الدراسة يمكن أن يكون لها تطبيقات والتي قد تؤدي إلى تطور ات إيجابية في رعاية المرضى وتعليم الممرضون و الأطباء والإداريين في الرعاية الصحية. نأمل بأنك سوف تو افق على المشاركة في هذه الدر اسة. مرفق مع ورقة المعلومات هذه هو استمارة مو افقة والتي

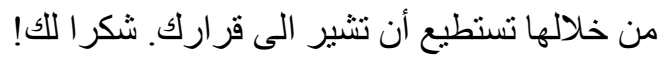




\section{Appendix 5 Participant Consent Form English}

Participant Identification Number:

\section{CONSENT FORM}

PROJECT TITLE: Exploring the perspectives of nurses, physicians, and healthcare administrators in Saudi Arabian hospitals on palliative care and palliative care nursing

Name of Researcher: Fhaied Almobarak

\section{Please initial box}

1. I confirm that I have read and understand the information sheet dated XXXX for the above study. I have had the opportunity to

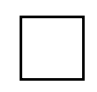
consider the information, ask questions and have had these answered satisfactorily.

2. I agree to take part in the study

3. I agree to participate in the interview

4. I consent to have my interview audio-recorded

5. I consent to have quotes from my interview included in reports or presentations

6. I understand that my participation is voluntary and that I am free to withdraw at any time, without giving any reason.

7. I understand that any information given by me may be used in future reports, articles or presentations by the research team.

8. I understand that my name will not appear in any reports, articles or presentations.

Name of Participant Date Signature

Researcher Date Signature

When completed, please return in the envelope provided (if applicable). One copy will be given to the participant and the original to be kept in the file of the research team at: Manchester Metropolitan University 


\section{Appendix 6 Participant Consent Form Arabic}

رقم تحديد المشارك:

\section{استمارة الموافقة}

عنوان المشروع : استكثاف تصورات ومفاهيم الممرضين والأطباء ومديري الرعاية الصحية في المستثفيات السعودية حول الرعاية التلطيفية وتمريض الرعاية التلطيفية

اسم الباحث: فهيد المبارك

يرجى تأثنيز المربع

1. أنا أقر بأنني قرأت وفهمت ورقة المعلومات المؤرخة في XXXX للاراسة أعلاه. وقد أتيحت لي الفرصة للنظر

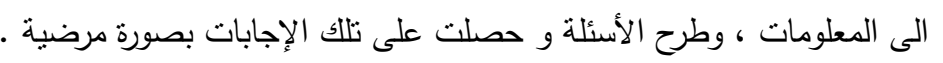

\begin{tabular}{|l|}
\hline \\
\hline$\square$ \\
\hline$\square$ \\
\hline$\square$ \\
\hline$\square$ \\
\hline
\end{tabular} 2. أنا أوافق على المشاركة في الدراسة 3. أوافق على المشاركة في المقابلة 4. - أنا موافق أن يكون صوت الدقابلة مسجل 5. أنا موافق أن تدرج اقتباسات ومقتطفات من المقابلة التي أجريتها في التقارير أو العروض التقديمية 6. وأنا أفهم أن مشاركتي هي طوعية وأنني حر في الانسحاب في أي وقت ، دون إبداء أب سبب. 7. وأنا أفهم أن أي معلومات قدمت مني يمكن استخدامها في التقارير المقبلة المستقبلية ، والمقالات أو العروض التقديمية بواسطة فريق البحث. 8. وأنا أفهم أن اسمي لن يظهر في أية تقارير ومقالات أو عروض تقدية لقيمية.

التوقيع التوقيع
التاريخ

التاريخ
اسم المشارك الباحث

عند الانتهاء، يرجى ارجاع الظرف المقدم ( إن وجد) . سوف تعطى نسخة واحدة إلى المشارك و الأصل سيبقى في الملف مع فريق البحث في: جامعة مانشستر مترويوليتان 


\section{Appendix 7 Interview Guide English}

Interview Guide

Date:.....

Time:.....

Location:....

Welcome and thank you for your participation today. My name is Fhaied Almobarak and I am a PhD student at Manchester Metropolitan University-UK, conducting my Special Study in partial fulfilment of the requirements for the degree of Doctorate of Philosophy in Palliative Care. This interview will take about $\mathbf{3 0}$ minutes and will include questions regarding your views about palliative care and specialising in palliative care, along with your experiences in relation to the delivery of palliative care to patients in the hospital that you are working at.

I would like your permission to voice record this interview, so I may accurately document the information you convey. If at any time during the interview you wish to discontinue the use of the recorder or the interview itself, please feel free to let me know. All of your responses are confidential. Your responses will remain confidential and will be used to develop a better understanding of how you and your other healthcare practitioners' colleague view palliative care and the specialisation into it

The purpose of this study is to determine the current state of palliative care in Saudi Arabia from the perspective of its healthcare professionals. Specifically, the research questions of the study are: (1) What are the perceptions of KSA hospital healthcare practitioners regarding palliative care? (2) What issues regarding the development of palliative care are evident in the KSA hospital setting? And (3) What recommendations can be made for strengthening the value of palliative care among healthcare practitioners in Saudi Arabia?

At this time I would like to remind you of your written consent to participate in this study. I am the responsible investigator, specifying your participation in the research project Palliative Care Perceptions of Healthcare Professionals in Saudi Arabia. You and I have both signed and dated each copy, certifying that we agree to continue this interview. You will receive one copy and I will keep the other under lock and key, separate from your reported responses. Thank you.

Your participation in this interview is completely voluntary. If at any time you need to stop, please let me know. You may also withdraw your participation at any time without consequence. Do you have any questions or concerns before we begin? Then with your permission we will begin the interview.

Main Questions, Additional Questions, Clarifying Question

Can you give a brief description of your work responsibilities? 
What is your understanding of palliative care?

Have you ever experienced palliative care for family member?

Have they been looked after?

How is palliative care handled?

What role do faith or spirituality play in the patient's and/or family's beliefs and what influence do they have on behaviour?

What role does the spiritual provider play with respect to the dying patient?

In what way do you think that spiritual care is applied?

How do you deal with the cultural differences?

Can you tell me about pain medication (Morphine)?

How do you deal when diagnosing the patient with for example cancer?

What are the palliative organisations (association)?

What are the legislation and constitution regarding palliative care in KSA?

What role do media play in palliative care?

What do you think about careers in palliative care?

What can you say about people who specialize or want to specialize in palliative care?

How do you perceive palliative care as a specialization for a nurse or physician?

Would you consider specializing in palliative care?

Are these nurses specially trained for palliative care?

Are there any training opportunities provided to hospital staff on palliative care?

How developed would you say is palliative care in your hospital?

Are there any specific issues with regard to palliative care in your hospital?

Do you have any other comments on the issue of palliative care?

Do you have any suggestion or recommendations for the future?

Would you advise and recommend that palliative care as an essential measure in all hospitals?

Is there anything else you wish to add?

Are you happy with the interview? 
Thank you for your time and participation

In addition, a tentative time frame after which the report would be published and would be available for you to read and so we assure you that your anonymity as a participant in the study will be maintained at all times. You will not be personally identified in any of our records that relate directly to your transcript, and your contact information will be discarded after we deliver a copy of the interview transcript to you for verification. 


\section{Appendix 8 Interview Guide Arabic}

دليل المقابلة

تاريخ:.

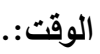

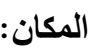

أرحب بك وشكرا للك على المشاركة اليوم. اسمي فهيد المبارك و أنا طالب دكتوراه في جامعة مانشستر منروبوليتان في المملكة المتحدة - بريطانيا ، وأنا أقوم بإجراء دراستي وذلك للاستيفاء الجزئي لمتطلبات الحصول على درجة الدكتوراه في الفلسفة في الرعاية التلطيفية ــ وذه المقابلة تستغرق حوالي 40 دقيقة، و سوف تشمل أسئلة بخصوص وجهات نظركم حول الرعاية التلطيفية وحول التخصص في الرعاية النطيفية ، جنبا إلى جنب مع خبراتكم فيما يتعلق بتقديم الرعاية التلطيفية للمرضى في المستشفى الذي تعمل فيه.

أود الحصول على إذن منك لتسجيل هذه المقابلة ، وذلك يمكنني من التوثيق بدقة للمعلومات التي تتقلها. إذا كنت في أي وقت خلال المقابلة ترغب في توقيف استخدام التسجيل أو المقابلة نفسها ، لا تتردد في ذلك ودعني أعرف ـ كل ردودك واجاباتك سرية. ستبقى ردودك سرية و سيتم استخدامها لنطوير الفهم الأفضل للك ولزملائك المتخصصين في الرعاية الصحية حول كيفية النظر الى الرعاية التلطيفية والتخصص في الرعاية التلطيفية.

الغرض من هذه الدراسة هو تحديد الوضع الحالي للرعاية التلطفية في المملكة العربية السعودية من وجهة نظر المتخصصين في الرعاية الصحية. على وجه التحديد ، الأسئلة البحثنة للدراسة هي : ( 1 ) ما هي تصورات ممارسي الرعاية الصحية في مستشفيات المملكة العربية السعودية بخصوص الرعاية التلطيفية والتخصص في الرعاية التلطيفية ؟ ( 2 ) ما هي القضايا فيما يتعلق بنطوير الرعاية التلطيفية والتي هي واضحة في المستشفيات السعودية ؟و (3 ) ما هي التوصيات التي يمكن أن تتم لتعزيز قيمة الرعاية التلطيفية بين ممارسي الرعاية الصحية في المملكة العربية السعودية؟ في هذا الوقت أود أن أذكرك في الموافقة الخطية للمشاركة في هذه الدراسة. أنا المحقق السوؤول ، وتحديد مشاركتك في المشروع البحثي تصورات الرعاية التلطيفية من المتخصصين في الرعاية الصحية في المملكة العربية السعودية. أنت وأنا على حد سواء وضعنا توقيع وتاريخ في كل نسخة ، تتبت أننا نتقق على مواصلة هذه المقابلة . سوف تتلقى نسخة واحدة و سأبقي الأخرى في درج مقفل ، معزوله عن ردودك. شكرا للك. 
مشاركتلك في هذه المقابلة هي طوعيه تماما ـ إذا كنت في أي وقت تحتاج للتوقف ، أرجوا أن تعلمني بذلك. يمكنك أيضا سحب مشاركناك في أي وقت دون عواقب ـ هل لديك أب أسئلة أو استقسارات قبل أن نبدأ ؟ اذن بعد إذنكم سنبدأ

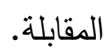

أسئلة رئيسية ، أسئلة إضافية ، وتوضيح السؤال

1. هل يمكناك ان تعطيني وصفا موجزا لمسؤوليات عملك؟

2.

3.

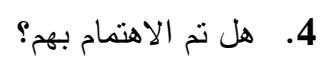

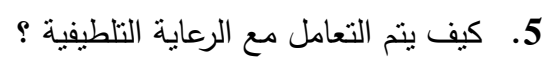

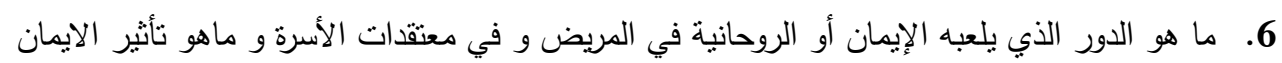

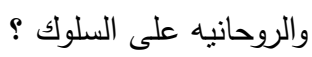

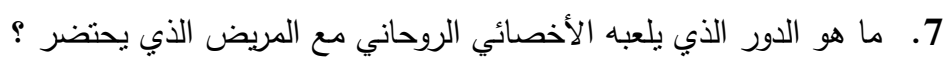

8.

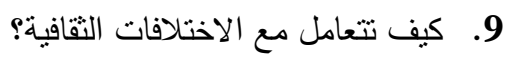

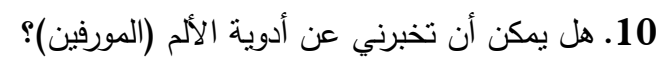

11. كيف تتعاملون عند تشخيص المريض مع مثنلا السرطان

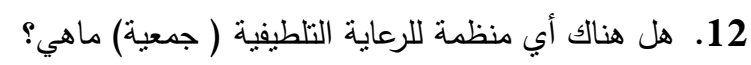

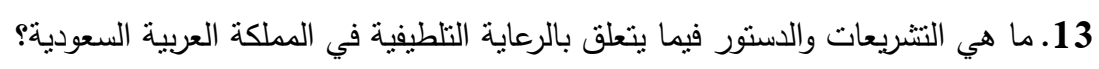

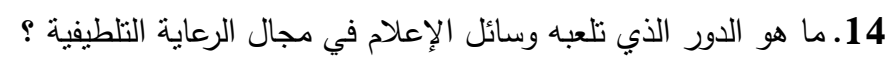

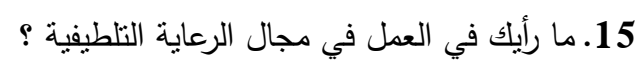

16. ماذا يمكن أن تقول عن الناس الذين يتخصصون أو بريدون التخصص في مجال الرعاية التلطيفية؟

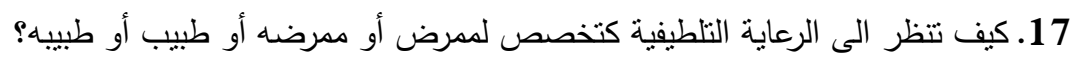

18. هل تفكر في التخصص في الرعاية التلطيفية ؟

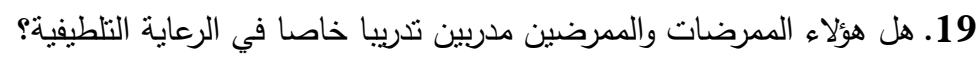

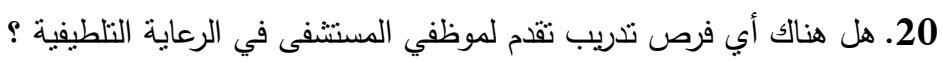

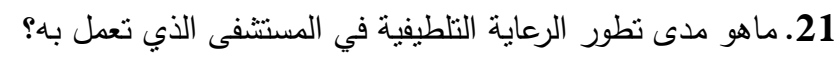

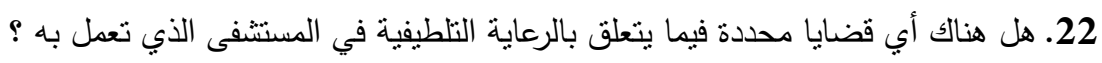

23. هل لايك أي نعليقات أخرى بشأن مسألة الرعاية النلطيفية ؟

24. هل لديك أي اقتراح أو توصيات للمستقبل ؟

25. هل تتصح و نوصي بأن تصبح الرعاية التلطيفية كتدبير أساسي ومعيار يجب أن يطبق في جميع المستشفيات

26. هل هناك أي شيء آخر ترغب في إضافته ؟

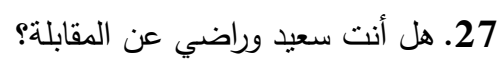


شكرا للك على وقتك والمشاركة

بالإضافة إلى ذلك، فإن هناك اطار وقت مبدئي وبعد ذلك سيتم نشر التقرير و سيكون متاح للك للقراءة و لذا فإننا نؤكد لكم أن هوينك كمشارك في الدراسة سيتم الاحتفاظ بها في جميع الأوقات. فلن يتم التعريف بك شخصيا في أي من سجلاتتا التي تتصل مباشرة إلى نص المقابلة الخاص بك، و سوف ينم التخلص من معلومات الاتصال الخاصة بك بعد مانقوم بنسليم نسخة للك من محضر المقابلة للتحقق منه. 